\title{
Mapeamentos Simpléticos EM DINÂMICA ASTEROIDAL
}

\author{
Fernando Virgilio Roig
}

\author{
Dissertação de Mestrado \\ submetida ao \\ Departamento de Astronomia \\ Instituto Astronômico e Geofísico \\ Universidade de São Paulo
}

Orientador: Sylvio Ferraz Mello

São Paulo, 1997 

A mis sobrinos

Gisela y Agustín, y a mi ahijado Franco 



\section{Resumo}

Neste trabalho, desenvolvemos um mapeamento simplético que nos permite estudar o comportamento dinâmico de ressonâncias asteroidais no âmbito do problema dos três corpos restrito, elíptico, espacial. Para obter este mapeamento, combinamos um esquema simplético similar ao desenvolvido por Hadjidemetriou (1986) junto com um desenvolvimento assimétrico da função perturbadora (Ferraz-Mello, 1987), que leva em conta as inclinações do perturbado e do perturbador como sendo referidas a um plano invariante (Roig et al., 1997). Este mapeamento é aplicado aos casos das ressonâncias asteroidais 2/1 e 3/2. Estudam-se um grande número de condições iniciais no espaço de fase, de forma a conseguir tirar conclusões de tipo estatístico sobre os processos envolvidos na geração dos mecanismos difusivos que podem agir nessas ressonâncias. 


\begin{abstract}
In this work, we develope a symplectic mapping which allows us to study the dynamical behaviour of asteroidal resonances in the frame of the non-planar elliptic restricted three-body problem. To obtain such a mapping we combine a symplectic scheme similar to that of Hadjidemetriou (1986) together with an asymmetric expansion of the disturbing function (Ferraz-Mello, 1987) which takes into account the inclinations of both the perturber and the disturbed bodies (Roig et al., 1997). This mapping is applied to the $2 / 1$ and $3 / 2$ mean motion resonances in the asteroidal belt. We explore a wide range of initial conditions in the phase space in order to get a large number of results which allows us to make some statistical conclusions about the generation of diffusion mechanisms acting in these resonances.
\end{abstract}




\section{PRÓLOGO}

...To see the world in a grain of sand,

And the sky in a wild flower,

To hold the infinity in the palm of the hand,

And the eternity in an hour...

W. Blake, "Songs of inocence"

Quando comecei a escrever esta dissertação não tinha idéia de que iria ficar tão comprida. Porém, acho que ela reflete muito bem todo o que eu apreendi nestes dois anos e meio de estudo e trabalho. Há muitas pessoas às quais devo agradecer por ter me ajudado a concretizar esta dissertação, tanto no aspecto acadêmico quanto no aspecto pessoal. Dentre elas, quero mencionar especialmente:

A Sylvio Ferraz Mello por ter me ensinado o verdadeiro significado de fazer pesquisa em

Mecânica Celeste, e pela amizade e confiança que me brindou durante este tempo tudo. Não poderia ter desejado um melhor orientador.

A Cristián Beaugé, que me convenceu de vir fazer a pós-graduação no IAG. Sem seu encorajamento, com certeza meu Mestrado nem teria começado.

A Alessandro Simula, pela amizade, a parceria, e pelas longas horas de estudo que compartilhamos enquanto misturávamos desenvolvimentos assimétricos com macarrão italiano. 
A David Nesvorný, amigo além das diferenças de linguagem e sempre disposto a esclarecer as dúvidas mais intrincadas (como o significado da palavra tcheca "děkuji").

Aos "hispano-hablantes" do $g D S^{2}$ : Tabaré Gallardo, Felipe Wachlin, Pablo Cincotta e suas respectivas famílias, por fazer-me sentir como se nunca tivesse ido embora de Argentina.

A Tatiana Michtchenko por facilitar dados muito valiosos que serviram para melhorar a apresentação das figuras da dissertação; a Julio Klafke pelas diversas sugestões sobre o desenvolvimento assimétrico; e aos Profs. Masa e Rama, pelos excelentes cursos que me ministraram.

A Alejandro Dimarco, por muitas mais coisas das que podem entrar nestas páginas de agradecimentos: se tentasse fazer uma lista não acabaria mais.

A Juan Pablo Neirotti, por ter facilitado minha adaptação durante as primeiras semanas que passei em São.

A Andrea Sánchez, por toda a "buena onda" e amizade que me mandou pelo e-mail desde Montevideo.

A todos aqueles amigos (brasileiros, argentinos, chilenos, etc.) que conheci aqui no Brasil e que, de uma forma ou outra, contribuíram para que me sentisse integrado ao meio.

Aos muitos amigos que ficaram na Argentina, por não ter me esquecido apesar da distância.

Às secretárias do IAG e da CPG, por estar sempre dispostas a resolver os problemas mais variados; e ao pessoal da biblioteca, por todas as xerox que tirou para mim.

À FAPESP e à CAPES pelo apoio econômico fornecido através das respectivas bolsas, e sem o qual, a possibilidade de vir estudar no IAG não teria passado de ser um sonho.

Para concluir, quero agradecer muito especialmente a minha família pelo apoio permanente, e em particular a minha mãe por ter a paciência para esperar, a inteligência para compreender, e a fé necessária para rezar por mim. 


\section{Conteúdo}

Prólogo

Conteúdo vii

1 Introdução 1

$\begin{array}{llr}2 & \text { Mapeamentos simpléticos } & \mathbf{7}\end{array}$

2.1 Conceitos básicos . . . . . . . . . . . . . . 7

2.2 Mapeamentos como aproximações de fluxos Hamiltonianos . . . . . . . 8

2.3 Mapeamentos explícitos . . . . . . . . . . . . . . . 11

2.4 Mapeamentos implícitos . . . . . . . . . . . . . . . . . . . . . 14

2.5 Forma Hamiltoniana de um mapeamento . . . . . . . . . . . . . 17

2.6 Comentários . . . . . . . . . . . . . . . . . . . . . 21

3 O modelo aplicado $\quad 25$

3.1 As variáveis do problema . . . . . . . . . . . . 25

3.1 .1 Unidades . . . . . . . . . . . . . . . . . . . 28

3.2 A função perturbadora . . . . . . . . . . . . . . . . 28

3.3 o mapeamento . . . . . . . . . . . . . . . . 33

3.4 As derivadas da Hamiltoniana . . . . . . . . . . . . . . . . . . 34

3.4.1 Derivadas do desenvolvimento assimétrico médio . . . . . . . 35

3.4.2 Derivadas das variáveis do desenvolvimento . . . . . . . . 36 
4 Variações de longo período da órbita do perturbador 39

4.1 Teorias seculares sintéticas . . . . . . . . . . . . . . . 39

4.2 Perturbações seculares consideradas no trabalho . . . . . . . . . . . 41

4.3 Perturbações críticas de curto período . . . . . . . . . . . . . . 42

4.3.1 Precisão das expressões para as variações de curto período . . . . 45

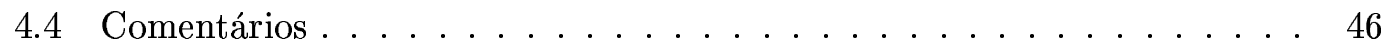

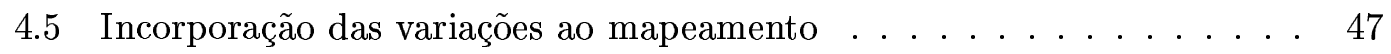

$\begin{array}{llr}5 & \text { Resultados } & 49\end{array}$

5.1 Análise dos resultados . . . . . . . . . . . . . . . 49

5.2 A ressonância $2 / 1 \ldots \ldots \ldots \ldots \ldots \ldots \ldots \ldots$

$5.3 \quad$ A ressonância $3 / 2 \ldots \ldots \ldots \ldots \ldots \ldots \ldots$

6 Conclusões e perspectivas futuras $\quad 93$

$\begin{array}{ll}\text { Bibliografia } & 95\end{array}$

$\begin{array}{ll}\text { A Conceitos básicos sobre ressonâncias asteroidais } & 103\end{array}$

A.1 A dinâmica do problema ressonante . . . . . . . . . . . . . 103

A.2 As ressonâncias $2 / 1$ e $3 / 2 \ldots \ldots \ldots$. . . . . . . . . . 107

A.2.1 A ressonância $2 / 1 \ldots \ldots \ldots \ldots$. . . . . . . . . . . . . . .

A.2.2 A ressonância $3 / 2 \ldots \ldots \ldots \ldots \ldots \ldots$

$\begin{array}{ll}\text { B Lista dos asteróides reais utilizados } & 117\end{array}$

I The high-eccentricity asymmetric expansion of the disturbing func$\begin{array}{ll}\text { tion for non-planar resonant problems } & 121\end{array}$ 


\section{INTRODUÇÃO}

O estudo da dinâmica asteroidal ressonante apresenta duas dificuldades maiores: (1) trata de um problema com muitos graus de liberdade e mesmo os modelos mais simples não são integráveis; (2) a evolução orbital secular é muito lenta e, em muitos casos importantes, fica fora do alcance das simulações numéricas precisas. A partir do trabalho pioneiro de Poincaré, os astrônomos têm tentado evitar essas dificuldades utilizando diferentes aproximações baseadas em métodos de média para eliminar as oscilações de alta freqüência do problema, isto é, os termos de curto período da Hamiltoniana. O estudo do comportamento das equações médias resultantes tem ajudado a entender a dinâmica ressonante em períodos curtos de tempo, mas de um modo geral, todos os estudos numéricos e analíticos, têm ficado longe de desvendar a dinâmica em uma escala de tempo cosmogônica.

Os primeiros resultados numéricos com uma escala de tempo maior do que $10^{5}$ anos foram obtidos por Wisdom $(1982,1983)$, não utilizando métodos de média precisos ou integrações numéricas, mas por meio de um mapeamento aproximado que permitia uma velocidade de cálculo umas 100 vezes maior do que as integrações numéricas comuns. Assim, enquanto no ano '82 uma integração numérica de um asteróide na ressonância $3 / 1$ levava por volta de uma hora para cobrir um intervalo de tempo de 10,000 anos, o mapeamento de Wisdom conseguia cobrir uns 200,000 anos em menos tempo. Este mapeamento, baseado no mapa "standard" de Chirikov - Taylor (Chirikov, 1979), permitiu a Wisdom descobrir que um asteróide na ressonância $3 / 1$ podia apresentar difusão 
caótica, com intermitências ou pulos nos quais a excentricidade podia atingir valores maiores do que 0.35 , fazendo com que a órbita do asteróide cruzasse a órbita de Marte, e assim, pudesse ser tirado da sua órbita por possíveis colisões com esse planeta.

Estes mapeamentos algébricos não conseguem predizer a órbita do objeto com uma grande precisão, mas são muito mais rápidos que as integrações numéricas precisas e, então, permitem fazer predições sobre intervalos de tempo muito grandes e sobre um conjunto muito grande de condições iniciais, fato que é essencial em um estudo numérico de movimentos asteroidais ressonantes. Na verdade, o movimento de um asteróide é governado por uma Hamiltoniana que depende do tempo e que possui três graus de liberdade. Isto significa que existe um espaço de fase multidimensional que deve ser varrido com condições iniciais, e assim os mapeamentos resultam muito mais eficientes que as integrações numéricas para acessar grandes domínios no espaço de fase. Em geral, estas pesquisas têm um caráter só exploratório e, como foi feito por Wisdom no caso da ressonância 3/1, uma vez que os fenômenos importantes têm sido descobertos, devem utilizar-se integrações numéricas precisas para confirmar sua existência real. A partir do trabalho de Wisdom, muitos problemas da astronomia dinâmica têm sido tratados utilizando diferentes tipos de mapeamentos (cf. Capítulo 2). Em particular, Hadjidemetriou $(1986,1988,1991)$ desenvolveu um método para a construção de um mapeamento algébrico aproximando um sistema dinâmico que se acha próximo de um sistema integrável. Essa aproximação considera primeiro o problema integrável com seu correspondente mapeamento "twist" em uma superfície de seção adequada. Logo, esse mapeamento "twist" é perturbado de forma a incluir várias das características mais importantes do sistema não integrável, que podem ser conhecidas previamente mediante estudos analíticos e numéricos. Mais explicitamente, o mapeamento de Hadjidemetriou é construído de forma a possuir os mesmos pontos fixos, com os mesmos índices de estabilidade, que a superfície de seção do sistema médio.

O mapa de Hadjidemetriou lhe permitiu obter as superfícies de seção da ressonância $3 / 1$, reproduzindo todas as características conhecidas dessa ressonância. O mapa reproduz a região caótica interna que é responsável pelas intermitências achadas por Wisdom. Além do mais, após a introdução de certo termo corretivo na Hamiltoniana, Hadjidemetriou (1993) obteve a região caótica externa da ressonância 3/1 responsável pelas oscilações de muito alta excentricidade, como assim também a ponte que permite 
a um asteróide passar da região caótica interna à região externa em escalas de tempo da ordem de um milhão de anos. Estas características das librações de alta excentricidade, primeiramente descobertas por Ferraz-Mello e Klafke (1991), permitem às excentricidades atingir valores perto de 0.9, fazendo com que o asteróide caia para o sistema solar interior, cruzando as órbitas da Terra e de Vênus.

O mapeamento de Hadjidemetriou é por construção um mapeamento simplético, e é equivalente a um integrador simplético de primeira ordem. Ele pode ser considerado como semelhante ao mapa de Wisdom já que, de acordo com Yoshida (1993), o mapa de Wisdom é também um integrador simplético de primeira ordem, além do fato de serem esquemas diferentes já que o primeiro é um mapa implícito enquanto o segundo é explícito.

O mapeamento de Wisdom foi aplicado com muito sucesso por Šidlichovský e Melendo (1986) e por Šidlichovský $(1992,1993)$ para estudar a ressonância asteroidal 5/2 e outras ressonâncias de ordem superior. No entanto, a extensão do mapa de Wisdom para ressonâncias de baixa ordem como a $2 / 1$ ou $3 / 2$, obteve só um sucesso relativo (Murray, 1986). Como já foi destacado por Murray, a extensão do mapa de Wisdom para as ressonâncias $2 / 1$ e $3 / 2$ possui dificuldades pelo fato de que o desenvolvimento laplaciano clássico da função perturbadora nessas ressonâncias é insuficiente. A porcentagem de erro em um desenvolvimento até segunda ordem nas excentricidades é só $12 \%$ para uma excentricidade $e=0.3$ no caso da ressonância $3 / 1$, mas atinge $35 \%$ no caso da ressonância $2 / 1$ e $74 \%$ no caso da $3 / 2$. De fato, a situação é ainda pior, devido a que, no caso destas duas ressonâncias, uma excentricidade de 0.3 fica fora do raio de convergência do desenvolvimento clássico da função perturbadora. Para a ressonância 2/1, o raio de convergência na excentricidade é 0.2 e para a 3/2, 0.09 (Ferraz-Mello, 1994a).

Devido às limitaçoes na convergência do desenvolvimento laplaciano clássico, particularmente no caso das ressonâncias asteroidais mais externas, qualquer mapeamento baseado neste desenvolvimento resulta ser valido só para excentricidades muito baixas. Como já mencionamos, Hadjidemetriou (1993) consegue evitar este problema adicionando no desenvolvimento clássico, um termo corretivo que é deduzido empiricamente. No entanto, a utilização de outros tipos de desenvolvimentos pode ser explorada. Em particular, Ferraz-Mello (1997) propôs uma versão modificada do mapeamento de 
Hadjidemetriou para fazer um estudo das ressonâncias 2/1 e 3/2. Esta versão é formalmente igual ao mapa de Hadjidemetriou mas, ao invés do desenvolvimento clássico, a função perturbadora é representada pelo desenvolvimento assimétrico (Ferraz-Mello, 1987, 1989; Ferraz-Mello e Sato, 1989). A grande diferença entre o desenvolvimento clássico e este último desenvolvimento é que, no primeiro, a expansão é uma série de potências em torno da origem, isto é, ao redor do valor $e=0$, enquanto que no segundo, a série de Taylor é desenvolvida em torno de centros de libração genéricos, isto é, pontos do espaço nos quais $e \neq 0$. Assim, o desenvolvimento assimétrico constitui um modelo mais preciso do potencial perturbador em domínios do espaço onde a excentricidade é muito grande.

Em principio, não existe uma razão para dizer que o desenvolvimento assimétrico esteja isento de problemas de convergência, ou que a sua convergência seja melhor do que a convergência do desenvolvimento laplaciano. Ao contrario, o raio de convergência deve ser menor quanto maior é a excentricidade do centro do desenvolvimento. Assim, os mapeamentos baseados neste tipo de desenvolvimento também ficam limitados por problemas de convergência. Mas estas limitações podem ser ignoradas se o movimento do asteróide fica perto do centro do desenvolvimento, isto é, se a excentricidade e o ângulo ressonante não atingem valores muito além dos adotados para esse centro. Assim, um mapeamento que utilize o desenvolvimento assimétrico resulta ser um modelo ótimo para a descrição do movimento de um asteróide nas regiões onde as librações de alta excentricidade apresentam amplitudes moderadas.

Num modelo restrito, a órbita de Júpiter é mantida fixa no espaço. Portanto, a única ressonância secular existente é aquela associada com a precesão do periélio do asteróide. Esta ressonância é responsável pela grande região de corrotação que é observada no meio da ressonância 2/1 para altas excentricidades (Ferraz-Mello, 1994b). Quando o movimento real do periélio de Júpiter é introduzido, levando em conta as suas variações seculares mais importantes, a região de corrotação fica quase inalterada devido a que o periélio de Júpiter se mexe muito devagar. Mas a inclusão dessas variações aumenta o número de graus de liberdade do problema, e esse aumento faz com que agora, regiões caóticas que não existiam quando Júpiter era mantido em uma órbita fixa, se tornem aparentes. Trabalhos recentes de Morbidelli e Moons (1993) e de Henrard et al. (1995) mostram que uma difusão orbital importante em uma ressonância 
só é conseguida quando se introduzem as variações seculares da órbita de Júpiter. Como exemplos temos a região caótica de alta excentricidade associada com o "overlap" das ressonâncias seculares $\nu_{5}$ e $\nu_{6}$, e a região caótica associada com o "overlap" destas duas ressonâncias com a ressonância de Kozai.

No estudo recentemente desenvolvido por Ferraz-Mello (1997) utilizando o mapa de Hadjidemetriou conjuntamente com o desenvolvimento assimétrico, o modelo considerado é plano, isto é, Júpiter e o asteróide possuem órbitas co-planares. A órbita de Júpiter não é considerada como sendo fixa, mas levam-se em conta suas variações seculares mais importantes (Nobili et al., 1989) devidas às perturbações dos planetas exteriores, como também os termos de curto período devidos à quase comensurabilidade 5/2 existente entre Júpiter e Saturno (Simon e Bretagnon, 1975). Os principais resultados do trabalho de Ferraz-Mello mostram que no caso da ressonância 2/1, as perturbações de curto período na órbita de Júpiter, geradas pela comensurabilidade 5/2 com Saturno, são mais importantes que as perturbações seculares, e evidenciam processos difusivos que levam a escapes em escalas de tempo da ordem de $10^{7} \sim 10^{9}$ anos. Isto significa que a estocasticidade global observada em modelos de 4 corpos que incluem Saturno, é gerada pelas principais perturbações na órbita de Júpiter, e particularmente, pelas perturbações de curto período. Assim, a falta de sucesso dos modelos baseados em métodos de média em mostrarem um comportamento caótico forte na região da ressonância $2 / 1$ pode ser devida ao fato de que as perturbações de longo período não possuem a capacidade de gerar esse caos. No entanto, as soluções obtidas para o caso da ressonância $3 / 2$ não apresentam nenhum comportamento difusivo importante. Um detalhe a ser destacado é que os mecanismos planos são muito mais lentos do que outros mecanismos observados em experimentos numéricos tri-dimensionais feitos por Wisdom (1987) e por Henrard et al. (1995). Neste trabalho de tese visamos estender estes resultados de Ferraz-Mello, tentando introduzir melhoras significativas no modelo.

Por um lado, utilizaremos o desenvolvimento assimétrico da função perturbadora adaptado para levar em conta os termos correspondentes às inclinações do perturbado e do perturbador (Roig et al., 1997). A utilização deste desenvolvimento espacial permitirá desvendar a possível importância da inclusão das perturbações não planares na geração de processos difusivos. Por outro lado, introduziremos expressões mais realistas para calcular a perturbação de curto período na órbita de Júpiter devida à ressonância 
5/2 com Saturno. As expressões utilizadas no trabalho de Ferraz-Mello só são válidas por um intervalo de tempo de uns poucos milhares de anos; neste trabalho utilizaremos expressões genéricas cuja validade pode estender-se por intervalos de tempo alguns ordens de grandeza mais longos.

Com base no desenvolvimento assimétrico espacial, serão montadas as equações do mapeamento, utilizando o esquema simplético de Hadjidemetriou. Com este mapeamento exploraremos um grande número de condições iniciais nas ressonâncias $2 / 1 \mathrm{e}$ $3 / 2$ por intervalos de tempo de $\sim 10^{8}$ anos, tentando determinar, por um lado, que processos difusivos atuam nas diferentes regiões do espaço de fase e, por outro, como influencia a perturbação devida à quase-ressonância $5 / 2$ entre Júpiter e Saturno na aceleração dos processos difusivos. A idéia principal é obter um primeiro conjunto de resultados qualitativos, que seja estatisticamente significativo, como permitir determinar os aspectos do problema sobre os que valeria a pena, num futuro próximo, fazer pesquisas mais detalhadas, seja por intervalos de tempo maiores ( $\sim 10^{9}$ anos), seja utilizando metodologias mais precisas.

O que resta desta tese está organizado da seguinte forma. O Capítulo 2 apresenta os fundamentos teóricos básicos dos mapeamentos simpléticos. Os Capítulos 3 e 4 descrevem o modelo e metodologia utilizados ao longo do trabalho. O Capítulo 5 é dedicado a analisar os resultados da pesquisa. Um último capítulo é reservado às conclusões e perspectivas futuras. No Apêndice A apresentamos uma breve introdução ao estudo das ressonâncias de movimentos médios, e resumimos as principais características dinâmicas das ressonâncias asteroidais $2 / 1$ e 3/2. Finalmente, algumas tabelas e alguns detalhes extremamente técnicos da metodologia aplicada são apresentados no Apêndice B e no Anexo I. 


\section{Mapeamentos Simpléticos}

Apresentamos neste capítulo os conceitos básicos sobre mapeamentos simpléticos aplicados ao estudo da dinâmica do Sistema Solar. Alguns "reviews" sobre o tema podem ser achados consultando Froeschlé (1992), Yoshida (1993), Hadjidemetriou (1996), Šidlichovský (1996) ou Henrard (1997).

\subsection{Conceitos básicos}

Consideremos um sistema Hamiltoniano com $n$ graus de liberdade, cujo espaço de fase constitui uma variedade $2 n$-dimensional $M$, descrita localmente pelas coordenadas $q_{1}, \ldots, q_{n}$ e pelos momentos $p_{1}, \ldots, p_{n}$. A evolução temporal deste sistema é dada por um grupo monoparamétrico de difeomorfismos $\Phi^{t}: M \rightarrow M$ chamado fluxo, com a regra de composição $\Phi^{t+s}=\Phi^{t} \circ \Phi^{s}$. Este fluxo possui a propriedade de preservar a estrutura simplética natural da variedade $M$ que é dada pela 2 -forma diferencial

$$
\omega^{2}=\sum_{i} d p_{i} \wedge d q_{i}
$$

Isto significa que a 2-forma $\omega^{2}$ é um invariante integral do sistema, ou, em outras palavras, que qualquer volume $2 n$-dimensional de condições iniciais em $M$ é conservado ao longo da evolução do sistema (teorema de Liouville). O fluxo $\Phi^{t}$ é chamado simplético.

Em termos gerais, simplético é sinônimo de canônico. A propriedade de preservar a estrutura simplética pode ser expressada da seguinte forma: Uma transformação 
$(\mathbf{q}, \mathbf{p}) \rightarrow\left(\mathbf{q}^{\prime}, \mathbf{p}^{\prime}\right)$ é simplética (canônica) se a sua matriz Jacobiana $\mathfrak{M}=\partial\left(\mathbf{q}^{\prime}, \mathbf{p}^{\prime}\right) / \partial(\mathbf{q}, \mathbf{p})$ cumpre com a propriedade

$$
\mathfrak{M}^{\mathrm{T}} \mathbf{J} \mathfrak{M}=\mathbf{J}
$$

sendo $\mathbf{J}$ a matriz simplética antissimétrica

$$
\mathbf{J}=\left(\begin{array}{cc}
\mathbf{0} & \mathbf{I} \\
-\mathbf{I} & \mathbf{0}
\end{array}\right)
$$

onde $\mathbf{0}$ e I são as matrizes $n \times n$ nula e identidade, respectivamente. Uma conseqüência imediata desta propriedade é que

$$
(\operatorname{det} \mathfrak{M})^{2}=1
$$

e, mais precisamente, det $\mathfrak{M}=1$ (Wintner, 1941), ou seja, a transformação simplética preserva o volume. É importante destacar que alguns autores (Kinoshita el al., 1991; Yoshida, 1992) utilizam a propriedade da Eq. (2.4) como definição de simplético; no entanto isto é errado já que essa é uma condição necessária mas não suficiente.

\subsection{Mapeamentos como aproximações de fluxos Hamiltonianos}

Consideremos o operador derivada de Lie $\mathcal{L}_{G}$ definido a partir do colchete de Poisson

$$
\mathcal{L}_{G} F=[F, G]=F_{\mathbf{q}} G_{\mathbf{p}}-F_{\mathbf{p}} G_{\mathbf{q}}
$$

Logo, as equações de movimento de um sistema Hamiltoniano cuja Hamiltoniana é $\mathcal{H}$, podem ser escritas na forma

$$
\frac{d}{d t}(\mathbf{q}, \mathbf{p})=\mathcal{L}_{\mathcal{H}}(\mathbf{q}, \mathbf{p})
$$

Formalmente, o fluxo $\Phi^{t}$ deste sistema é representado pelo operador série de Lie

$$
\Phi^{t}=\exp t \mathcal{L}_{\mathcal{H}}=\sum_{i=0}^{\infty} \frac{t^{i}}{i !} \mathcal{L}_{\mathcal{H}}^{i}
$$

e a evolução do sistema desde $(\mathbf{q}, \mathbf{p})$ no instante $t$ até $\left(\mathbf{q}^{\prime}, \mathbf{p}^{\prime}\right)$ no instante $t+\tau$ é dada por

$$
\left(\mathbf{q}^{\prime}, \mathbf{p}^{\prime}\right)=\exp \tau \mathcal{L}_{\mathcal{H}}(\mathbf{q}, \mathbf{p})
$$

Isto é uma transformação simplética por definição, já que o operador série de Lie representa uma transformação canônica infinitesimal. 
A forma mais simples de construir um mapeamento que represente aproximadamente o fluxo Eq. (2.7) é truncar a solução Eq. (2.8) em alguma ordem $k$. Assim por exemplo, um mapeamento de primeira ordem possui a forma

$$
\begin{aligned}
q_{i}^{\prime} & =q_{i}+\tau\left[q_{i}, \mathcal{H}\right]+\mathcal{O}\left(\tau^{2}\right) \\
p_{i}^{\prime} & =p_{i}+\tau\left[p_{i}, \mathcal{H}\right]+\mathcal{O}\left(\tau^{2}\right)
\end{aligned}
$$

e o erro que cometemos a cada passo é $\mathcal{O}\left(\tau^{2}\right)$ (isto é basicamente o integrador numérico de Euler). Logo, para garantir que nos manteremos perto do fluxo real precisamos $\tau \ll 1$. No entanto, o fato de truncar a série de Lie faz com que este mapeamento não seja simplético e, mesmo que possamos escolher um passo muito pequeno, os invariantes integrais do problema não serão conservados. Um exemplo típico disto é o oscilador harmônico linear

$$
\mathcal{H}(p, q)=\frac{1}{2}\left(p^{2}+q^{2}\right)
$$

que é um sistema integrável e cujas trajetórias são círculos. Um mapeamento de primeira ordem fornece a solução

$$
\begin{aligned}
& q^{\prime}=q+\tau p \\
& p^{\prime}=p-\tau q
\end{aligned}
$$

onde $\tau$ é o passo do mapeamento. Logo, é simples mostrar que

$$
\mathcal{H}\left(p^{\prime}, q^{\prime}\right)=\left(1+\tau^{2}\right) \mathcal{H}(p, q)
$$

portanto, a energia não é conservada a cada passo e as trajetórias são espirais, isto é, o erro da solução apresenta um crescimento secular com o tempo. Muitos integradores numéricos, ainda que de alta ordem, possuem este tipo de desvantagem, e seria desejável, portanto, que o mapeamento fosse simplético, de forma a conseguir que se conservem os invariantes integrais do problema. As Eqs. (2.11) podem ser modificadas para obter um mapeamento simplético de primeira ordem, escrevendo

$$
\begin{aligned}
q^{\prime} & =\left(1-\tau^{2}\right) q+\tau p \\
p^{\prime} & =p-\tau q
\end{aligned}
$$

É simples ver que este mapeamento conserva a cada passo a grandeza $\frac{1}{2}\left(p^{2}+q^{2}-\tau p q\right)$, logo, as trajetórias não são círculos mas elipses com excentricidade constante $e=$ 
$\sqrt{\frac{2 \tau}{2+\tau}} \cdot{ }^{1}$ A Hamiltoniana Eq. (2.10) não é conservada a cada passo mas as trajetórias não espiralam. Assim para valores pequenos do passo $\tau$ o mapeamento simplético representa qualitativamente bem o comportamento do oscilador harmônico.

Em geral, qualquer mapeamento simplético não consegue preservar exatamente os invariantes integrais do problema, já que de uma ou outra forma a construção do mapeamento está baseada no truncamento, numa certa ordem $k$, da série de Lie que descreve o fluxo do problema. No entanto, a simpleticidade garante que são conservadas certas grandezas muito próximas dos invariantes integrais. Portanto, o erro da solução fornecida pelo mapeamento não apresenta uma variação secular com o tempo, e fica limitado às ordens $\mathcal{O}\left(\tau^{k+1}\right)$. É claro que se o sistema é integrável, sempre é possível achar um mapeamento simplético que preserve exatamente todas as integrais de movimento. Nesse caso o mapeamento é a solução exata do problema.

Uma forma muito utilizada para estudar a evolução de um fluxo consiste em analisar o comportamento do sistema sobre superfícies de energia constante, e contruir uma superfície de seção de forma que o espaço de fase $2 n$-dimensional fique reduzido a um subespaço $(2 n-2)$-dimensional onde a analise é mais simples. Este sub-espaço é conhecido como mapa de Poincaré. Os pontos fixos do mapa de Poincaré correspondem às órbitas periódicas do problema e sua estabilidade caracteriza localmente a topologia do espaço de fase. O problema é que para construir estes mapas de Poincaré precisamos conhecer "a priori" o fluxo do sistema para poder determinar sua interseção com a superfície de seção. Alternativamente podemos tentar construir um mapeamento algébrico que reproduza aproximadamente este fluxo sobre a superfície de seção. Para que este mapeamento seja realístico três condições devem ser cumpridas (Hadjidemetriou, 1996): (1) o mapeamento deve ser simplético; (2) o mapeamento deve possuir os mesmos pontos fixos que o mapa de Poincaré; (3) os índices de estabilidade dos pontos fixos do mapeamento e do mapa de Poincaré devem ser os mesmos ${ }^{2}$. Voltaremos sobre isto mais

\footnotetext{
${ }^{1}$ Mais precisamente, se

$$
\frac{1}{2}\left(p^{2}+q^{2}-\tau p q\right)=\overline{\mathcal{H}}
$$

os semieixos $a, b$ da elipse são tais que

$$
\tau=2 \frac{a^{2}-b^{2}}{a^{2}+b^{2}} ; \quad \overline{\mathcal{H}}=\frac{a^{2} b^{2}}{a^{2}+b^{2}} .
$$

${ }^{2} \mathrm{O}$ índice de estabilidade de um ponto fixo é definido como a soma dos autovalores das soluções
} 
tarde.

Analisaremos a seguir diferentes tipos de esquemas simpléticos utilizados na literatura e a forma em que são construídos.

\subsection{Mapeamentos explícitos}

Existem muitos exemplos de sistemas Hamiltonianos em que a Hamiltoniana pode ser separada em duas partes. O mais comum é aquele em que uma das partes corresponde à energia cinética $T$ e a outra à energia potencial $V$ :

$$
\mathcal{H}=T(\mathbf{p})+V(\mathbf{q})
$$

mas também há aqueles em que uma das partes é integrável e a outra é uma perturbação dependente de um pequeno parâmetro $\varepsilon$ :

$$
\mathcal{H}=\mathcal{H}_{0}(\mathbf{q}, \mathbf{p})+\mathcal{H}_{1}(\mathbf{q}, \mathbf{p}, \varepsilon) \quad \varepsilon \ll 1
$$

Em qualquer destes casos é possível mostrar que o operador série de Lie Eq. (2.7) pode ser escrito na forma

$$
\exp \tau \mathcal{L}_{\mathcal{H}}=\exp \tau\left(\mathcal{L}_{\mathcal{H}_{0}}+\mathcal{L}_{\mathcal{H}_{1}}\right)=\exp \tau \mathcal{L}_{\mathcal{H}_{0}} \exp \tau \mathcal{L}_{\mathcal{H}_{1}}+\mathcal{O}\left(\tau^{2}\right)
$$

Agora, o operador $\exp \tau \mathcal{L}_{\mathcal{H}_{0}} \exp \tau \mathcal{L}_{\mathcal{H}_{1}}$ constitui um mapeamento de primeira ordem que é simplético, pois é a composição de duas transformações canônicas infinitesimais. Percebamos que o operador $\exp \tau \mathcal{L}_{\mathcal{H}_{1}} \exp \tau \mathcal{L}_{\mathcal{H}_{0}}$ também gera um mapeamento simplético de primeira ordem que é formalmente equivalente ao anterior mas é algebricamente diferente. Este tipo de mapeamento foi formalizado e estendido às ordens maiores por Yoshida (1990), Forest e Ruth (1990) e Forest el al. (1991). Algumas aplicações em diferentes problemas foram feitas por Candy e Rozmus (1991), Kinoshita et al. (1991) e Kinoshita e Nakai (1992).

Uma propriedade muito importante da série de Lie é dada pela identidade de Baker - Campbell - Haussdorf (Varadarajan, 1974). Se os operadores $\mathcal{L}_{\mathcal{H}_{0}}$ e $\mathcal{L}_{\mathcal{H}_{1}}$ são tais que

$$
\left\langle\mathcal{L}_{\mathcal{H}_{0}}, \mathcal{L}_{\mathcal{H}_{1}}\right\rangle=\mathcal{L}_{\mathcal{H}_{0}} \mathcal{L}_{\mathcal{H}_{1}}-\mathcal{L}_{\mathcal{H}_{1}} \mathcal{L}_{\mathcal{H}_{0}} \neq 0
$$

das equações variacionais em torno do ponto fixo. O índice de estabilidade de uma órbita periódica é definido como o traço da matriz monodrômica da órbita periódica. 
(o símbolo $\langle$,$\rangle representa o comutador dos operadores), então podemos escrever$

$$
\exp \tau \mathcal{L}_{\mathcal{H}_{0}} \exp \tau \mathcal{L}_{\mathcal{H}_{1}}=\exp \tau \mathcal{L}_{\overline{\mathcal{H}}}
$$

onde

$$
\mathcal{L}_{\overline{\mathcal{H}}}=\mathcal{L}_{\mathcal{H}_{0}}+\mathcal{L}_{\mathcal{H}_{1}}+\frac{\tau}{2}\left\langle\mathcal{L}_{\mathcal{H}_{0}}, \mathcal{L}_{\mathcal{H}_{1}}\right\rangle+\mathcal{O}\left(\tau^{2}\right)
$$

O operador $\exp \tau \mathcal{L}_{\overline{\mathcal{H}}}$ descreve então o fluxo de um sistema cuja Hamiltoniana é

$$
\overline{\mathcal{H}}=\mathcal{H}_{0}+\mathcal{H}_{1}+\frac{\tau}{2}\left[\mathcal{H}_{0}, \mathcal{H}_{1}\right]+\mathcal{O}\left(\tau^{2}\right)
$$

e cuja diferença com a Hamiltoniana original $\mathcal{H}$ é da ordem de $\tau$. Assim, o mapeamento simplético de primeira ordem Eq. (2.18) preserva não a Hamiltoniana $\mathcal{H}$ mas $\overline{\mathcal{H}}$. É claro que para um mapeamento de ordem $k$ a diferença entre $\mathcal{H}$ e $\overline{\mathcal{H}}$ é da ordem $\mathcal{O}\left(\tau^{k}\right)$. Particularmente interessante é o caso em que na Eq. (2.15) $\mathcal{H}_{1}(\mathbf{q}, \mathbf{p}, \varepsilon)=\varepsilon \mathcal{H}_{1}(\mathbf{q}, \mathbf{p})$. Nesse caso temos que $\mathcal{H}-\overline{\mathcal{H}} \approx \mathcal{O}\left(\tau^{k} \varepsilon\right)$ e o passo $\tau$ pode ser escolhido $\varepsilon^{-1 / k}$ vezes maior mantendo a mesma ordem de diferença entre as duas Hamiltonianas.

Rigorosamente, a Eq. (2.20) escreve-se como sendo

$$
\overline{\mathcal{H}}=\mathcal{H}+\sum_{k=1}^{\infty} \tau^{k} P_{k}\left(\mathcal{H}_{0}, \mathcal{H}_{1}\right)
$$

onde cada coeficiente $P_{k}\left(\mathcal{H}_{0}, \mathcal{H}_{1}\right)$ é uma dada combinação linear dos colchetes de Poisson

$$
\left[\cdots[\left[\mathcal{H}_{i}, \mathcal{H}_{j}\right] \underbrace{\left.\left.\cdots \mathcal{H}_{i} \cdots\right] \cdots \mathcal{H}_{j} \cdots\right]}_{k-1 \text { vezes }} \quad i, j=0,1 .\right.
$$

Se esta série de potências de $\tau$ não é convergente, então acontece que $\overline{\mathcal{H}}$ não é integrável, ainda que $\mathcal{H}$ seja integrável. Logo, o mapeamento pode quebrar a integrabilidade do sistema original. Um exemplo clássico em que isto acontece é o caso do pêndulo, que é integrável; porém, o mapeamento vinculado ao pêndulo, isto é, o mapa "standard", não é integrável e apresenta faixas de comportamento caótico perto das separatrizes, que se tornam desprezíveis para valores pequenos de $\tau$, mas que mesmo assim não desaparecem.

O esquema simplético definido pelo operador Eq. (2.18) resulta particularmente útil quando nos achamos no caso de um sistema separável como a Eq. (2.14), isto é, onde uma parte da Hamiltoniana só depende dos momentos e a outra só das coordenadas. Nesse caso as séries $\exp \tau \mathcal{L}_{T}(\mathbf{q}, \mathbf{p})$ e $\exp \tau \mathcal{L}_{V}(\mathbf{q}, \mathbf{p})$ possuem um número finito de termos. 
Logo, o mapeamento simplético de primeira ordem pode ser escrito, por exemplo, na forma

$$
\begin{aligned}
q_{i}^{\prime} & =q_{i}+\tau \frac{\partial T(\mathbf{p})}{\partial p_{i}} \\
p_{i}^{\prime} & =p_{i}-\tau \frac{\partial V\left(\mathbf{q}^{\prime}\right)}{\partial q_{i}^{\prime}}
\end{aligned}
$$

Quando a Hamiltoniana não é separável, as séries de Lie possuem infinitos termos, e, para efeitos práticos, elas devem ser truncadas numa certa ordem, o que quebra a simpleticidade do mapeamento.

Uma propriedade interessante deste mapeamento é que ele não depende do conjunto de variáveis canônicas que é utilizado, já que o colchete de Poisson é independe do tipo de variáveis utilizado. Kinoshita et al. (1991) exploram esta propriedade para aplicar o esquema precisamente em Hamiltonianas que não são separáveis num mesmo conjunto de variáveis, mas que podem sê-lo em conjuntos diferentes. Um exemplo típico é a Hamiltoniana do problema de $N$-corpos. Utilizando coordenadas cartesianas e momentos $(r, p)$ podemos escrever

$$
\mathcal{H}=\mathcal{H}_{0}(p, r)+\varepsilon \mathcal{R}(r)
$$

onde $\mathcal{R}(r)$ é a função perturbadora. No entanto, utilizando variáveis ação é ângulo $(L, \ell)$ podemos escrever

$$
\mathcal{H}=\mathcal{H}_{0}(L)+\varepsilon \mathcal{R}(L, \ell)
$$

Logo, o mapeamento pode ser dividido em dois passos: primeiro aplicamos o operador $\exp \tau \mathcal{L}_{\mathcal{R}}$

$$
\begin{aligned}
& r^{*}=r \\
& p^{*}=p-\tau \varepsilon \frac{\partial \mathcal{R}}{\partial r} ;
\end{aligned}
$$

a seguir transformamos $\left(r^{*}, p^{*}\right) \rightarrow\left(\ell^{*}, L^{*}\right)$, e finalmente aplicamos o operador $\exp \tau \mathcal{L}_{\mathcal{H}_{0}}$

$$
\begin{aligned}
\ell^{\prime} & =\ell^{*}+\tau \frac{\partial \mathcal{H}_{0}}{\partial L^{*}} \\
L^{\prime} & =L^{*} .
\end{aligned}
$$

É importante dizer que este mapa combinado só é simplético se a transformação $(r, p) \leftrightarrow$ $(\ell, L)$ é simplética. 


\subsection{Mapeamentos implícitos}

Uma outra forma de construir um mapeamento simplético baseia-se em achar uma função geratriz da transformação canônica. Assim, o mapeamento $(\theta, J) \rightarrow\left(\theta^{\prime}, J^{\prime}\right)$ fica definido pelas equações implícitas

$$
\begin{aligned}
\theta & =\theta^{\prime}+\frac{\partial S}{\partial J^{\prime}} \\
J^{\prime} & =J+\frac{\partial S}{\partial \theta}
\end{aligned}
$$

onde $S\left(\theta, J^{\prime}\right)$ é uma função geratriz de Jacobi. Destaquemos que $\theta$ e $J$ são variáveis ângulo e ação e que, em geral, este tipo de esquema não pode ser aplicado com um conjunto de variáveis canônicas arbitrário. Consideremos por exemplo um sistema integrável $\mathcal{H}_{0}(J)$. A solução deste sistema é

$$
\begin{aligned}
J^{\prime} & =J \\
\theta^{\prime} & =\theta+\tau \frac{\partial \mathcal{H}_{0}}{\partial J}
\end{aligned}
$$

e constitui um mapeamento chamado "twist". Este mapeamento é gerado pela função geratriz

$$
S\left(\theta, J^{\prime}\right)=\theta J^{\prime}+\tau \mathcal{H}_{0}\left(J^{\prime}\right)
$$

e representa uma transformação quase-identidade para $\tau \ll 1$. Agora, vamos supor que perturbamos o sistema adicionando à Hamiltoniana um termo $\varepsilon H_{1}(\theta, J)$. Então a função geratriz da transformação fica

$$
S\left(\theta, J^{\prime}, \varepsilon\right)=\theta J^{\prime}+\tau\left[\mathcal{H}_{0}\left(J^{\prime}\right)+\varepsilon \mathcal{H}_{1}\left(\theta, J^{\prime}\right)\right]
$$

( $\varepsilon$ entra como um parâmetro da transformação), e o mapeamento é

$$
\begin{aligned}
J^{\prime} & =J-\tau \varepsilon \frac{\partial \mathcal{H}_{1}\left(\theta, J^{\prime}\right)}{\partial \theta} \\
\theta^{\prime} & =\theta+\tau \nu\left(J^{\prime}\right)+\tau \varepsilon \frac{\partial \mathcal{H}_{1}\left(\theta, J^{\prime}\right)}{\partial J^{\prime}}
\end{aligned}
$$

onde $\nu=\partial \mathcal{H}_{0} / \partial J^{\prime}$ é a freqüência não perturbada (Hadjidemetriou, 1986). Percebamos que estas fórmulas são parecidas àquelas do integrador de Euler (Eqs. 2.9); logo, o mapeamento é de primeira ordem. Este esquema pode ser estendido a problemas com vários graus de liberdade em forma muito simples através da geratriz

$$
S\left(\boldsymbol{\theta}, \mathbf{J}^{\prime}\right)=\sum_{i} \theta_{i} J_{i}^{\prime}+\tau \mathcal{H}\left(\boldsymbol{\theta}, \mathbf{J}^{\prime}\right)
$$


O mapeamento assim obtido é simplético por construção. Percebamos que a Hamiltoniana não necessariamente precisa ser da forma $\mathcal{H}_{0}+\varepsilon \mathcal{H}_{1}$, e a geratriz Eq. (2.33) pode ser montada para qualquer tipo de Hamiltoniana. No entanto, é importante destacar que $\mathcal{H}$ deve estar escrita em variáveis ação e ângulo, devido a que o esquema é construído tomando como base um mapeamento "twist". Isto acarreta certos incovenientes perto da origem $(J=0)$ já que as equações do mapeamento tornam-se singulares.

A principal desvantagem deste tipo de mapeamento é que ele é implícito nas ações. No caso particular em que $\mathcal{H}_{1}=\mathcal{H}_{1}(\theta)$ (sistema separável) ele pode ser explicitado trivialmente, constituindo o que é chamado de mapeamento "twist" radial, já que só as ações são perturbadas. Porém, no caso geral a equação nas ações deve ser resolvida por algum método numérico de aproximações sucessivas. É claro que o erro desta solução pode ser reduzido até a ordem de precisão da máquina. Por outro lado, uma alternativa para obter uma expressão analítica explícita consiste em utilizar um desenvolvimento em série de Lagrange (Whittaker e Watson, 1950), que é algebricamente equivalente a utilizar aproximações sucessivas. Para isto escrevemos a primeira Eq. (2.32) na forma

$$
J^{\prime}=J+\varepsilon f\left(J^{\prime}\right)
$$

e a solução desta equação é dada pela série

$$
J^{\prime}=J+\sum_{k} \frac{\varepsilon^{k+1}}{(k+1) !} \frac{\partial^{k}[f(J)]^{k+1}}{\partial J^{k}}
$$

que é convergente para valores pequenos de $\varepsilon$. No entanto, a manipulação destas séries de Lagrange torna-se muito complicada quando temos várias equações implícitas a serem resolvidas simultaneamente. Uma segunda alternativa consiste em transformar a função geratriz de Jacobi $S\left(\theta, J^{\prime}, \varepsilon\right)$ numa função geratriz de Lie que gere uma transformação canônica infinitesimal no parâmetro $\varepsilon$ e explícita em todas as variáveis. Segundo o procedimento clássico (Lanczos, 1974) escolhemos uma geratriz de Lie da forma

$$
B\left(\theta^{\prime}, J^{\prime}, \varepsilon\right)=\frac{\partial S\left(\theta, J^{\prime}, \varepsilon\right)}{\partial \varepsilon}=\tau \mathcal{H}_{1}\left(\theta, J^{\prime}\right)
$$

onde o ângulo $\theta$ nos segundo membros deve ser substituído por sua expressão em função de $\theta^{\prime}$, invertendo a segunda Eq. (2.32). A transformação canônica é então

$$
\begin{aligned}
\frac{d \theta^{\prime}}{d \varepsilon} & =\frac{\partial B\left(\theta^{\prime}, J^{\prime}, \varepsilon\right)}{\partial J^{\prime}} \\
\frac{d J^{\prime}}{d \varepsilon} & =-\frac{\partial B\left(\theta^{\prime}, J^{\prime}, \varepsilon\right)}{\partial \theta^{\prime}}
\end{aligned}
$$


e a solução destas equações pode ser expressada em termos das séries de Lie

$$
\begin{aligned}
\theta^{\prime} & =\theta_{0}+\sum_{k=1}^{\infty} \frac{\varepsilon^{k}}{k !}\left[\mathcal{L}_{B}^{k} \theta\right]_{\varepsilon=0} \\
J^{\prime} & =J_{0}+\sum_{k=1}^{\infty} \frac{\varepsilon^{k}}{k !}\left[\mathcal{L}_{B}^{k} J\right]_{\varepsilon=0}
\end{aligned}
$$

onde $\theta_{0}, J_{0}$ são os valores do mapeamento quando $\varepsilon=0$, isto é

$$
\begin{aligned}
& J_{0}=J \\
& \theta_{0}=\theta+\tau \nu(J) .
\end{aligned}
$$

A equivalência entre o mapeamento implícito (Eq. 2.32) e o explícito (Eq. 2.38) fica estabelecida a partir da equivalência entre as transformações de Jacobi e de Lie (Mersman, 1971). Porém, esta equivalência pode ser visualizada intuitivamente na Fig. 2.1. Com o mapeamento implícito passamos diretamente das variáveis $(\theta, J) \rightarrow\left(\theta^{\prime}, J^{\prime}\right)$ considerando $\varepsilon \neq 0$. No entanto com o mapeamento explícito passamos primeiro de $(\theta, J) \rightarrow\left(\theta_{0}, J_{0}\right)$ considerando $\varepsilon=0$ e depois passamos infinitesimalmente de $\left(\theta_{0}, J_{0}\right) \rightarrow$ $\left(\theta^{\prime}, J^{\prime}\right)$ considerando $\varepsilon \neq 0$. Em outras palavras, a passagem de $(\theta, J) \rightarrow\left(\theta_{0}, J_{0}\right)$ é equivalente a fazer evoluir o sistema segundo $\mathcal{H}_{0}$, enquanto que a passagem de $\left(\theta_{0}, J_{0}\right) \rightarrow$ $\left(\theta^{\prime}, J^{\prime}\right)$ é equivalente a fazer evoluir o sistema segundo $\mathcal{H}_{1}$. Logo o mapeamento Eq. (2.38) é conceitualmente equivalente ao mapeamento explícito definido pelo operador $\exp \tau \mathcal{L}_{\mathcal{H}_{1}} \exp \tau \mathcal{L}_{\mathcal{H}_{0}}$ que estudamos na seção anterior.

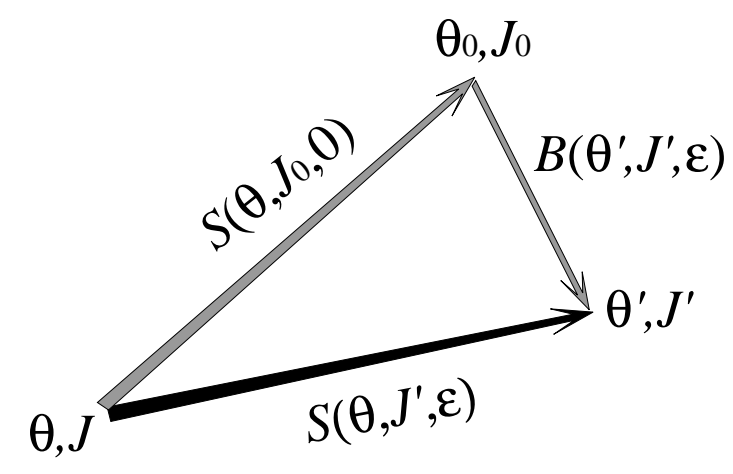

Figura 2.1: Equivalência entre as transformações de Jacobi e de Lie.

O esquema implícito foi primeiramente proposto por Ruth (1983) para ser aplicado em sistemas separáveis. Posteriormente, o esquema foi utilizado por Hadjidemetriou $(1986,1988,1991,1993)$ para estudar o comportamento de Hamiltonianas ressonantes 
médias no problema de três corpos. Em particular, Hadjidemetriou demonstrou a seguinte propriedade. Se $\langle\mathcal{H}\rangle=\left\langle\mathcal{H}_{0}\right\rangle+\varepsilon\left\langle\mathcal{H}_{1}\right\rangle$ é a Hamiltoniana que resulta de fazer a média de $\mathcal{H}$ sobre uma certa variável rápida cujo período é $T$, os pontos fixos do mapeamento de $\langle\mathcal{H}\rangle$ (Eq. 2.32) coincidem sempre com os pontos fixos do mapa de Poincaré de $\mathcal{H}$. Ainda mais, os índices de estabilidade dos pontos fixos do mapeamento coincidem até $\mathcal{O}\left(\varepsilon^{2}\right)$ com os índices de estabilidade dos pontos fixos do mapa de Poincaré quando o passo do mapeamento é escolhido como sendo $\tau=T$.

Digamos finalmente que é possível construir esquemas implícitos de alta ordem. Por exemplo, um esquema de segunda ordem é obtido a partir da função geratriz

$$
S\left(\boldsymbol{\theta}, \mathbf{J}^{\prime}\right)=\sum_{i} \theta_{i} J_{i}^{\prime}+\tau \mathcal{H}\left(\boldsymbol{\theta}, \mathbf{J}^{\prime}\right)+\frac{\tau^{2}}{2} \frac{\partial \mathcal{H}}{\partial \boldsymbol{\theta}} \frac{\partial \mathcal{H}}{\partial \mathbf{J}^{\prime}}
$$

Em particular, Channell e Scovel (1990) desenvolvem um esquema implícito até ordem seis; no entanto, a manipulação das derivadas de ordem superior de $\mathcal{H}$ torna-se muito complicada.

\subsection{Forma Hamiltoniana de um mapeamento}

Até agora, temos analisado a forma de construir mapeamentos simpléticos a partir de uma dada Hamiltoniana. Estes mapeamentos constituem uma aproximação do fluxo do sistema descrito por essa Hamiltoniana. Uma forma alternativa de construir um mapeamento simplético é partir de uma solução aproximada do fluxo real e tentar achar a forma Hamiltoniana dessa solução aproximada. Isto é, dado um mapeamento

$$
(q, p) \stackrel{M}{\longrightarrow}\left(q^{\prime}, p^{\prime}\right)
$$

encontrar a Hamiltoniana $\mathcal{H}_{M}$ tal que o mapeamento seja a solução exata do sistema de equações de Hamilton

$$
\begin{aligned}
\frac{d q}{d t} & =\frac{\partial \mathcal{H}_{M}}{\partial p} \\
\frac{d p}{d t} & =-\frac{\partial \mathcal{H}_{M}}{\partial q} .
\end{aligned}
$$

É claro que este mapeamento é simplético por definição. Também é claro que o mapeamento conserva a Hamiltoniana $\mathcal{H}_{M}$. Logo a questão é ver se $\mathcal{H}_{M}$ é suficientemente próxima da Hamiltoniana original $\mathcal{H}$. 
Consideremos novamente as Eqs. (2.32) no caso particular em que $\mathcal{H}_{1}=\mathcal{H}_{1}(\theta)$. Nesse caso, como já dissemos, o mapeamento torna-se explícito e podemos dividi-lo em duas partes

$$
\left.\begin{array}{l}
J^{*}=J-\tau \varepsilon \frac{\partial \mathcal{H}_{1}(\theta)}{\partial \theta} \\
\theta^{*}=\theta \\
J^{\prime}=J^{*} \\
\theta^{\prime}=\theta^{*}+\tau \frac{\partial \mathcal{H}_{0}\left(J^{*}\right)}{\partial J^{*}}
\end{array}\right\} \text { primeira parte }
$$

A forma Hamiltoniana deste mapeamento é simples. Denotemos $\theta=\theta(t), \theta^{\prime}=\theta(t+\tau)$, $J=J(t), J^{\prime}=J(t+\tau)$, e definamos $\tau=2 \pi / \Omega$. Logo, podemos mostrar que é possível escrever a Hamiltoniana do mapeamento como sendo

$$
\mathcal{H}_{M}(\theta, J, t)=\mathcal{H}_{0}(J)+\varepsilon \mathcal{H}_{1}(\theta) 2 \pi \delta_{2 \pi}(\Omega t)
$$

onde

$$
\delta_{2 \pi}(t)=\sum_{k=-\infty}^{\infty} \delta(t-2 \pi k)
$$

é uma série de funções delta de Dirac com período $2 \pi$, que cumpre com a propriedade

$$
\lim _{\epsilon \rightarrow 0} \int_{k \tau-\epsilon}^{k \tau+\epsilon} f(t) \delta_{2 \pi}(\Omega t) d t=f(\tau) \Omega^{-1} .
$$

Nesse caso, as equações do mapeamento escrevem-se na forma

$$
\begin{aligned}
\frac{d J}{d t} & =-\varepsilon \frac{\partial \mathcal{H}_{1}}{\partial \theta} 2 \pi \delta_{2 \pi}(\Omega t) \\
\frac{d \theta}{d t} & =\frac{\partial \mathcal{H}_{0}}{\partial J}
\end{aligned}
$$

e a integração no intervalo $[t, t+\tau)$ leva as Eqs. (2.43). Percebamos que a integração poderia ser feita no intervalo $(t, t+\tau]$ o que levaria a um mapeamento formalmente equivalente ao anterior mas algebricamente diferente. Seja qual for o caso, no intervalo $(t, t+\tau)$ o sistema evolui segundo a Hamiltoniana não perturbada $\mathcal{H}_{0}$ ( $J$ fica invariante) até o instante $t+\tau$ em que a perturbação $\mathcal{H}_{1}$ é aplicada instantaneamente ( $J$ faz um pulo através da função delta). Logo, este esquema é conceitualmente equivalente aos esquemas de primeira ordem estudados anteriormente.

O primeiro a aplicar este tipo de mapeamento foi Chirikov (1979) para construir um mapeamento que representasse a dinâmica do pêndulo: o mapa "standard". O 
esquema pode ser estendido para sistemas com $n$ graus de liberdade e para sistemas não separáveis definindo a Hamiltoniana

$$
\mathcal{H}_{M}(\boldsymbol{\theta}, \mathbf{J}, t)=\mathcal{H}_{0}(\mathbf{J})+\varepsilon \mathcal{H}_{1}(\boldsymbol{\theta}, \mathbf{J}) 2 \pi \delta_{2 \pi}(\Omega t)
$$

Além disso, ele não esta restrito a um conjunto de variáveis canônicas particulares. Foi precisamente neste esquema que se baseou Wisdom (1982) para obter seu mapeamento da ressonância asteroidal 3/1. Este tipo de mapeamento foi aplicado ao estudo das ressonâncias asteroidais de alta ordem por Šidlichovský $(1992,1993)$ e Šidlichovský e Melendo (1986).

Percebamos que este tipo de mapeamento descreve a solução de uma Hamiltoniana que não é autônoma. Ela depende do tempo pois apresenta um pulo a cada intervalo $\tau$. Isto está relacionado com o fato já mencionado de que os mapeamentos simpléticos de primeira ordem preservam uma Hamiltoniana que difere da Hamiltoniana real do problema em $\mathcal{O}(\tau)$. No entanto, isto pode ser analisado desde outro ponto de vista. A diferença entre $\mathcal{H}_{M}$ e a Hamiltoniana original $\mathcal{H}$ são os termos de curto período introduzidos pela série de funções delta. Lembremos que o desenvolvimento em série de Fourier da função delta periódica é

$$
\delta_{2 \pi}(t)=\frac{1}{2 \pi}\left(1+2 \sum_{k=1}^{\infty} \cos k t\right),
$$

e todos os termos têm período $2 \pi / k \leq 2 \pi$. Estes termos de curto período não afetam o comportamento secular do sistema, e ainda mais, a influência deles nos resultados do mapeamento pode ser removida através de uma aplicação adequada do método de Hori. Isto é conhecido como corretor simplético (Wisdom et al., 1994).

É importante mencionar o fato de que a aplicação do esquema das funções delta nem sempre leva a obter um mapeamento simplético (Henrard, 1997). Na verdade, devemos supor que a Hamiltoniana cumpre com certas condições de regularidade para garantir que o mapeamento será simplético. No entanto, a função delta não é suficientemente regular como para garantir em todos os casos que a solução de $\mathcal{H}_{M}$ seja simplética. Se a Hamiltoniana é separável, é claro que o esquema das funções delta é equivalente aos esquemas explícito e implícito já estudados, e portanto, o mapeamento é simplético. No entanto, se a Hamiltoniana não for separável o esquema das funções delta leva a obter um mapeamento não simplético. Analisaremos isto com dois exemplos. 
Exemplo 1: consideremos a Hamiltoniana

$$
\mathcal{H}=\frac{J^{2}}{2}+K(J) \cos \theta
$$

A aplicação do esquema implícito leva à obtenção do mapeamento

$$
\begin{aligned}
J^{\prime} & =J+\tau K\left(J^{\prime}\right) \sin \theta \\
\theta^{\prime} & =\theta+\tau J^{\prime}+\tau \frac{d K\left(J^{\prime}\right)}{d J^{\prime}} \cos \theta
\end{aligned}
$$

que é simplético por construção. No entanto o esquema das funções delta leva ao mapeamento

$$
\begin{aligned}
& J^{\prime}=J+\tau K(J) \sin \theta \\
& \theta^{\prime}=\theta+\tau J^{\prime}+\tau \frac{d K(J)}{d J} \cos \theta
\end{aligned}
$$

que não é simplético. Se $K$ não depende de $J$ os dois esquemas são equivalentes: com $\tau=1$ o resultado é o mapa "standard".

Exemplo 2: consideremos a Hamiltoniana

$$
\mathcal{H}_{M}=\frac{1}{2} p q^{2} \delta_{2 \pi}(t)
$$

As soluções entre $[t, t+2 \pi)$ são dadas por

$$
\begin{aligned}
q^{\prime} & =q\left(1+\frac{q}{2}\right) \\
p^{\prime} & =p(1-q)
\end{aligned}
$$

é temos o Jacobiano $\mathfrak{M}$ tal que

$$
\operatorname{det} \mathfrak{M}=1-q^{2} \neq 1
$$

o que mostra que a transformação não é simplética.

É normal na literatura achar que o esquema das funções delta é identificado com um esquema que consiste em separar a Hamiltoniana em diferentes partes, cada uma delas integráveis separadamente. O mapeamento então resulta da aplicação consecutiva das soluções exatas de cada uma das partes em que foi dividida a Hamiltoniana. Este último método é, nem mais nem menos, que o esquema explícito estudado na Seção 2.3 e leva sempre à obtenção de um mapeamento simplético. Portanto, a identificação deste esquema com o esquema das funções delta é errada (Henrard, 1997). Em particular o 
"esquema de funções delta" aplicado por Wisdom (1982), baseado na Eq. (2.48), não é propriamente um esquema de funções delta. Acontece que Wisdom tenta representar as funções delta na forma

$$
\delta_{2 \pi}(\Omega t)= \begin{cases}\lim _{\epsilon \rightarrow 0} \frac{1}{\epsilon}, \quad & \tau-\epsilon<t<\tau+\epsilon \\ 0, & \text { em qualquer outro caso }\end{cases}
$$

e o resultado que ele acaba obtendo é equivalente a separar a Hamiltoniana em várias partes integráveis e compor o mapeamento com as soluções dessas partes individuais ${ }^{3}$.

Particularmente interessante é a aplicação deste método feita por Wisdom e Holman (1992a,b) ao caso da Hamiltoniana de $N$-corpos

$$
\mathcal{H}=\mathcal{H}_{0}\left(\vec{r}_{i}, \vec{v}_{i}\right)+\mu \mathcal{R}\left(\vec{r}_{i}\right) \quad \mu \ll 1
$$

onde $\mathcal{H}_{0}$ é uma soma de Hamiltonianas de 2-corpos, $\mathcal{R}$ é o potencial perturbador dos corpos entre sí, e $\vec{r}_{i}, \vec{v}_{i}$ são coordenadas e momentos de Jacobi. O esquema utilizado por eles é equivalente à aplicação do operador

$$
\exp \frac{\tau}{2} \mathcal{L}_{\mathcal{H}_{0}} \exp \tau \mu \mathcal{L}_{\mathcal{R}} \exp \frac{\tau}{2} \mathcal{L}_{\mathcal{H}_{0}}
$$

e constitui um mapeamento simplético de segunda ordem, conhecido na literatura como "leap-frog". A aplicação do operador $\exp \frac{\tau}{2} \mathcal{L}_{\mathcal{H}_{0}}$ é substituída, no caso, diretamente pela solução do problema de dois corpos em coordenadas cartesianas fornecida pelo algoritmo das funções $f$ e $g$ de Gauss (Danby, 1988). Por sua vez, a aplicação de $\exp \tau \mu \mathcal{L}_{\mathcal{R}}$ é trivial, já que $\mathcal{R}$ não depende dos momentos ${ }^{4}$.

\subsection{Comentários}

Nos últimos dez anos, os mapeamentos simpléticos tem sido aplicados em diversos problemas da dinâmica do Sistema Solar. Citemos, por exemplo, os trabalhos de Gladman et al. (1991) e Saha e Tremaine (1992), aplicados à descrição da evolução ao

\footnotetext{
${ }^{3}$ Se Wisdom tivesse feito uma integração rigorosa da função delta, o mapeamento resultante não seria simplético, já que no seu caso a Hamiltoniana original não é separável.

${ }^{4} \mathrm{O}$ fato de utilizar coordenadas de Jacobi deve-se a que estas coordenadas são canônicas. Porém, o esquema pode também ser aplicado utilizando coordenadas cartesianas heliocêntricas, que, em geral, não são canônicas no problema de $N$-corpos não restrito.
} 
longo prazo de sistemas de $N$-corpos. Também foram feitas aplicações ao estudo das órbitas cometárias nos trabalhos de Petrosky e Broucke (1988) e Levinson e Duncan (1994). Finalmente, mapeamentos derivados de esquemas simpléticos foram aplicados em problemas com dissipação por Malhotra (1994) e Cordeiro et al. (1996).

No entanto, é importante dizer que, além dos esquemas simpléticos mencionados aqui, existem outras formas de construir mapeamentos que aproximem muito bem o comportamento dinâmico de um sistema. É o caso do mapeamento de Duncan et al. (1989), e os mapas sintéticos de Froeschlé e Petit (1990).

A chave principal na construção de qualquer mapeamento consiste em escolher adequadamente o modelo dinâmico que vai ser utilizado como ponto de partida. Se o modelo é ruim, os resultados que fornecerá o mapeamento, seja este simplético ou não, serão igualmente ruins. Um exemplo típico disto é o trabalho de Murray (1986), cujo mapeamento para as ressonâncias $2 / 1$ e 3/2 estava baseado num desenvolvimento da função perturbadora não válido para excentricidades altas. Contrariamente, temos como exemplo o mapeamento de Hadjidemetriou (1993) da ressonância 3/1, onde o autor adiciona "ad-hoc", a partir de considerações empíricas, alguns termos corretivos na função perturbadora que tornam o mapeamento adequado para estudar o comportamento nas muito altas excentricidades.

Tanto o esquema aplicado por Wisdom (1982) na ressonância 3/1 quanto o aplicado por Hadjidemetriou (1993), baseados ambos no desenvolvimento Laplaciano da função perturbadora, fornecem resultados similares sobre o comportamento dos objetos nas baixas excentricidades. Num trabalho recente, Ferraz-Mello e Roig (1996) mostram que de fato, os dois esquemas são algebricamente idênticos até a primeira ordem no pequeno parâmetro $\mu$ da perturbação. Diferenças aparecem nos termos $\mathcal{O}\left(\mu^{2}\right)$ que não são importantes já que, na Hamiltoniana utilizada, a média é feita só até primeira ordem em $\mu$ e, além disso, ela é truncada em $\mathcal{O}\left(e^{2}\right) \sim \mathcal{O}(\mu)$.

Os mapeamentos simpléticos resultam ser ferramentas muito úteis para a modelagem aproximada de sistemas dinâmicos por intervalos de tempo muito compridos. Eles se comportam, não tanto como integradores, mas como simuladores que conseguem reproduzir muito bem características dinâmicas globais de um dado sistema: pontos de equilíbrio, regiões de regularidade e caoticidade no espaço de fase, escalas de tempo de difusão e de instabilidade global, etc. É possível mostrar que eles conservam exa- 
tamente as integrais primeiras vinculadas às simetrias do problema, como o momento angular e o movimento do centro de massas, e ainda mais, os integradores simpléticos de ordem par preservam também as propriedades de reversibilidade temporal.

A grande diferença com os integradores numéricos comuns é que, no mapeamento, podemos escolher um passo de integração $\tau$ grande sem que isso introduza um crescimento secular no erro da solução. No entanto, devemos levar em conta que o mapeamento descreve o comportamento da Hamiltoniana $\overline{\mathcal{H}}$ que depende de $\tau$, portanto, a solução do mapeamento estará mais longe do comportamento real do sistema quanto maior for o passo.

No capítulo seguinte estudaremos como aplicar um destes esquemas simpléticos ao problema que desejamos estudar. 


\section{O MODELO APLICADO}

Vamos apresentar neste capítulo os detalhes do modelo utilizado na pesquisa desenvolvida, especificando a forma em que foi construído o mapeamento e os detalhes técnicos que foi necessário levar em conta na hora da sua aplicação.

\subsection{As variáveis do problema}

Começamos com a definição das variáveis do problema ressonante médio. Sejam $a, e$, $I, \ell, \omega, \Omega$ os elementos orbitais na notação clássica ( $\ell$ é a anomalia média), seja $n$ o movimento médio, e sejam $\varpi=\omega+\Omega$ e $\lambda=\ell+\varpi$. Vamos nos referir ao corpo perturbador utilizando variáveis linha. Definimos, então, as variáveis modificadas de Delaunay

$$
\begin{array}{lll}
\lambda & , & L=\sqrt{\mu a} \\
\varpi \quad & G-L=-L\left(1-\sqrt{1-e^{2}}\right) \\
\Omega \quad & H-G=-G(1-\cos I)=-2 G \sin ^{2} \frac{I}{2} \\
t & , & \Lambda
\end{array}
$$

onde $\mu$ é a constante de gravitação, e $\Lambda$ é o momento associado ao tempo. Logo, a Hamiltoniana do problema restrito de três corpos é

$$
\mathcal{H}=-\frac{\mu^{2}}{2 L^{2}}+\Lambda-\mu m^{\prime} R(L, G, H, \lambda, \varpi, \Omega, t),
$$

sendo $m^{\prime}$ a massa do corpo perturbador e $R$ a função perturbadora. 
Consideremos uma ressonância de movimentos médios entre o corpo perturbado e o perturbador da forma $(p+q) / p$. Definimos então as variáveis angulares ressonantes na forma

$$
\begin{aligned}
\psi & =\frac{p+q}{q} \lambda^{\prime}-\frac{p}{q} \lambda \\
\sigma & =\frac{p+q}{q} \lambda^{\prime}-\frac{p}{q} \lambda-\varpi \\
\sigma_{z} & =\frac{p+q}{q} \lambda^{\prime}-\frac{p}{q} \lambda-\Omega
\end{aligned}
$$

e também definimos a variável sinódica

$$
Q=\frac{\lambda-\lambda^{\prime}}{q}
$$

Queremos achar as ações $P, J, J_{z}$ e $\Phi$, conjugadas a estes quatro ângulos, e para isto, fazemos uma transformação canônica

$$
L d \lambda+(G-L) d \varpi+(H-G) d \Omega+\Lambda d t=P d \psi+J d \sigma+J_{z} d \sigma_{z}+\Phi d Q
$$

Levando em conta que $d \lambda^{\prime}=n^{\prime} d t$, chegamos ao seguinte resultado

$$
\begin{aligned}
L & =-\frac{p}{q}\left(P+J+J_{z}\right)+\frac{\Phi}{q} \\
G & =-J+L \\
H & =-J_{z}+G \\
\Lambda & =\frac{p+q}{q} n^{\prime}\left(P+J+J_{z}\right)-n^{\prime} \frac{\Phi}{q} .
\end{aligned}
$$

Assim, podemos escrever a Hamiltoniana $\mathcal{H}$ em função das ações e ângulos ressonantes. Como a variável sinódica $Q$ tem uma variação rápida em comparação com as outras, definimos a Hamiltoniana do problema médio de primeira ordem em $m^{\prime}$, como sendo

$$
\mathcal{H}^{*}=\frac{1}{2 \pi} \int_{0}^{2 \pi} \mathcal{H} d Q
$$

Logo, $\mathcal{H}^{*}$ não depende de $Q$ e o momento $\Phi=$ cte.. Redefinindo a origem de $L$ e da energia na forma

$$
L \rightarrow L-\frac{\Phi}{q} ; \quad \mathcal{H}^{*} \rightarrow \mathcal{H}^{*}+n^{\prime} \frac{\Phi}{q},
$$

o momento $\Phi$ desaparece de $\mathcal{H}^{*}$. Assim, conseguimos escrever a Hamiltoniana do problema médio como sendo

$$
\mathcal{H}^{*}=-\frac{\mu^{2}}{2 L^{2}}-\frac{p+q}{p} n^{\prime} L-\mu m^{\prime} R^{*},
$$


onde

$$
L=-\frac{p}{q}\left(P+J+J_{z}\right)
$$

e temos

$$
R^{*}=\frac{1}{2 \pi} \int_{0}^{2 \pi} R d Q
$$

que é a função perturbadora média. Finalmente, a partir das Eqs. (3.6), as ações do problema ressonante médio ficam definidas na forma

$$
\begin{aligned}
P & =-\frac{p+q}{p} L+H=-\sqrt{\mu a}\left[\frac{p+q}{p}-\sqrt{1-e^{2}} \cos I\right] \\
J & =L-G=\sqrt{\mu a}\left[1-\sqrt{1-e^{2}}\right] \\
J_{z} & =G-H=\sqrt{\mu a} \sqrt{1-e^{2}}(1-\cos I) .
\end{aligned}
$$

Introduzindo as variáveis regulares

$$
\begin{aligned}
k+i h & =e \exp i \sigma \\
v+i u & =\sin \frac{I}{2} \exp i \sigma_{z}, \quad(i=\sqrt{-1})
\end{aligned}
$$

é possível pensar em $R^{*}$ como sendo uma função da forma $R^{*}(\alpha, \psi, k, h, v, u)$, onde $\alpha=a / a^{\prime}$. Podemos logo estabelecer as seguintes relações:

$$
\begin{aligned}
\alpha & =\frac{L^{2}}{\mu a^{\prime}} \\
e & =B \sqrt{2 J} \\
\sin \frac{I}{2} & =D \sqrt{2 J_{z}}
\end{aligned}
$$

onde

$$
B=\sqrt{\frac{2 L-J}{2 L^{2}}}, \quad D=\sqrt{\frac{1}{4(L-J)}} .
$$

Uma propriedade importante da ação $P$ é que, no caso do problema restrito circular $\left(e^{\prime}=0\right)$, a função perturbadora média não depende do ângulo $\psi$; portanto $P$ tornase uma integral do movimento. A evolução do sistema acontece ao longo de uma certa superfície $P=$ cte, cujo valor depende das condições iniciais (cf. Apêndice A, Seção A.1). No problema elíptico $\left(e^{\prime} \neq 0\right) P$ não se conserva, mas se $e^{\prime}$ é pequena, o movimento do sistema acontece numa faixa mais ou menos estreita em torno de uma certa superfície $P=$ cte. Veremos daqui a pouco como fazer uso desta propriedade em nosso modelo. 


\subsubsection{Unidades}

Aos efeitos de montar um código com o mapeamento, é importante definir adequadamente as unidades a serem utilizadas. Como unidade de massa utilizaremos a massa solar $M_{\odot}$. A unidade de tempo será o ano juliano de 365.25 dias. Finalmente a unidade de distância será

$$
\frac{\mathrm{UA}}{(2 \pi)^{2 / 3}}
$$

Com estas unidades, a constante de gravitação fica definida como $\mu=1,{ }^{1}$ e a Hamiltoniana $\mathcal{H}^{*}$ escreve-se como sendo

$$
\mathcal{H}^{*}=\left[-\frac{1}{2 L^{2}}+\frac{p+q}{p} n^{\prime} L-m^{\prime} R^{*}\right](2 \pi)^{4 / 3}
$$

$\operatorname{com} L=\sqrt{a}$.

\subsection{A função perturbadora}

O principal problema na construção do nosso modelo consiste em achar uma representação adequada da Hamiltoniana para descrever as librações de alta excentricidade nas ressonâncias 2/1 e 3/2, no âmbito do problema não planar. Como sabemos, não é possível utilizar o desenvolvimento Laplaciano da função perturbadora, já que ele apresenta sérios problemas de convergência nessas ressonâncias (Ferraz-Mello, 1994a).

Um modelo certamente adequado para este tipo de estudo, é o desenvolvimento assimétrico para alta excentricidade da função perturbadora média de Ferraz-Mello (1987), e particularmente, a extensão ao caso do problema espacial, de Roig et al. (1997). Este desenvolvimento é uma expansão da função perturbadora $R$ em série de Taylor, em torno do ponto

$$
a=a_{0}, \quad k=k_{0}, \quad h=h_{0}, \quad I=0, \quad e^{\prime}=0, \quad I^{\prime}=0 .
$$

A expansão é truncada nas ordens adequadas em cada variável de forma a se ter um resto $\mathcal{O}\left(e^{\prime 3}\right)$, utilizando para isto as seguintes hipóteses

$\frac{\Delta a}{a^{\prime}} \sim \mathcal{O}\left(e^{\prime}\right), \quad \Delta k=\Delta h \sim \mathcal{O}\left(e^{\prime}\right), \quad \sin \frac{I}{2} \sim \mathcal{O}\left(\sqrt{e^{\prime}}\right), \quad \sin \frac{I^{\prime}}{2} \sim \mathcal{O}\left(e^{\prime}\right)$.

\footnotetext{
${ }^{1}$ Lembremos que $\mu=k^{2}$, sendo $k=0.01720209895 \frac{\mathrm{UA}^{3 / 2}}{\operatorname{dia} M_{\odot}^{1 / 2}}$.
} 
No final, cada coeficiente da expansão é mediado sobre a variável $Q$ resolvendo numericamente a integral da Eq. $(3.11)^{2}$. O resultado final é um polinômio com coeficientes constantes dependentes dos valores de $a_{0}, k_{0}, h_{0}$. Não nos estenderemos sobre os detalhes da obtenção deste desenvolvimento, remetendo o leitor ao Anexo I.

É importante dizer que no âmbito do problema planar, o desenvolvimento assimétrico não é o único modelo adequado para o estudo das librações de alta excentricidade, e, se quisermos, poderíamos utilizar outros tipos de desenvolvimentos. Como exemplos, temos o desenvolvimento de Yokoyama (1994) para altas excentricidades e amplitudes de libração grandes, e o desenvolvimento globalmente convergente de Beaugé (1996). Infelizmente, estes desenvolvimentos não foram ainda estendidos ao caso do problema não plano. Uma outra opção que poderia ser explorada é o cálculo exato da função perturbadora média em cada ponto do espaço de fase (Moons, 1993). Porém, aos efeitos de levar adiante uma integração numérica, este método resulta muito mais lento que a utilização de uma expansão polinomial onde os coeficientes médios são calculados só uma vez ao começo da integração.

A partir daqui, centraremos nossa atenção nas ressonâncias de primeira ordem, isto é, quando $q=1$. Nesses casos, sabemos que o argumento crítico $\sigma$ libra em torno de $0^{\circ}$ enquanto que a excentricidade libra em torno de um certo valor $e_{0}$. Portanto, para aplicar o desenvolvimento assimétrico neste tipo de ressonância, podemos escolher centros de desenvolvimento ao longo do eixo $k$, isto é, com $h_{0}=0$. A escolha deste ponto $\left(k_{0}, 0\right)$ não pode ser arbitrária e deve estar vinculada de alguma forma com a existência da lei de estrutura da ressonância (Ferraz-Mello, 1988) ${ }^{3}$. Em outras palavras, os centros de desenvolvimento mais adequados serão aqueles pontos sobre o eixo $k$ que se acham sobre o ramo pericêntrico da ressonância ou muito perto dele.

Para estimar a posição deste centro podemos fazer uso da propriedade da ação $P$ mencionada na seção anterior. A partir das condições iniciais $a_{\mathrm{ini}}, e_{\mathrm{ini}}$ calculamos

$$
P_{\text {ini }}=-\sqrt{\mu a_{\text {ini }}}\left[(p+1) / p-\sqrt{1-e_{\text {ini }}^{2}}\right],
$$

\footnotetext{
${ }^{2}$ Nos usamos em particular uma quadratura de Gauss de 10 pontos.

${ }^{3}$ Lembremos que a lei de estrutura diz que as soluções com amplitude de libração de $\sigma$ nula (os centros de libração), constituem uma família contínua de pontos no espaço de fase. No plano $a, e$ esta família de soluções estacionárias forma uma curva contínua que recebe o nome de ramo pericêntrico (cf. Apêndice A).
} 
então obtemos

$$
e_{0}=\left[1-\left((p+1) / p-P_{\mathrm{ini}} / \sqrt{\mu a_{0}}\right)^{2}\right]^{1 / 2}
$$

sendo $a_{0}=a_{\mathrm{c}}$ o semieixo crítico da ressonância

$$
a_{\mathrm{c}}=\mu^{1 / 3}\left(\frac{p}{p+1} \frac{1}{n^{\prime}}\right)^{2 / 3} .
$$

O ponto $\left(a_{0}, e_{0}\right)$ se acha muito perto do ramo pericêntrico, pelo menos para valores $e_{0}>0.1$. Assim, nosso centro de desenvolvimento é o ponto $a_{0}, k_{0}=e_{0}, h_{0}=0$. No entanto, como nosso modelo possui vários graus de liberdade, poderia acontecer que este centro de libração estimado a partir de uma integral do movimento do modelo plano circular fosse muito diferente do centro de libração real do sistema. Para isto podemos fazer uma ajuste iterativo do centro de desenvolvimento. Começando com a estimação calculada acima, integramos o sistema durante um certo intervalo de tempo $T$ e calculamos os valores médios das variáveis $a, k, h$ nesse intervalo. Estes valores médios $\langle a\rangle,\langle k\rangle,\langle h\rangle$ são utilizados como novo centro de desenvolvimento, os coeficientes do desenvolvimento assimétrico são recalculados, e inicia-se novamente a integração pelo tempo $T$. Este processo é repetido até conseguir uma certa convergência nos valores de $\langle a\rangle,\langle k\rangle,\langle h\rangle$. Em nossa aplicação, escolhemos $T=10^{5}$ anos e observamos que entre 4 e 6 iterações são suficientes para obter um resultado com precisão $<10^{-10}$. Em particular, verificamos que $\langle h\rangle \simeq 0$.

A expressão final do desenvolvimento assimétrico médio $R^{*}$ em torno de um ponto $\left(k_{0}, 0\right)$, aparece no Anexo I (eq. 53). Para uniformizar à notação, primeiro introduzimos as definições $\sigma^{\prime}=\psi-\varpi^{\prime}$ e $\sigma_{z}^{\prime}=\psi-\Omega^{\prime}$, e definimos variáveis regulares para o perturbador na mesma forma que nas Eqs. (3.13):

$$
\begin{aligned}
k^{\prime}+i h^{\prime} & =e^{\prime} \exp i \sigma^{\prime} \\
v^{\prime}+i u^{\prime} & =\sin \frac{I^{\prime}}{2} \exp i \sigma_{z}^{\prime}
\end{aligned}
$$


Logo, podemos re-escrever a função perturbadora média $\mathcal{R}^{*}$, na forma

$$
\begin{aligned}
R^{*}=\frac{1}{a^{\prime}}[ & \mathcal{R}_{0}^{*}+\mathcal{R}_{0 k}^{*}\left(k-k_{0}\right)+\mathcal{R}_{0 \alpha}^{*}\left(\alpha-\alpha_{0}\right) \\
& +\mathcal{R}_{1}^{*} k^{\prime}+\mathcal{R}_{3}^{*} \frac{1}{2}\left(v^{2}+u^{2}\right)+\mathcal{R}_{4}^{*} \frac{1}{2}\left(v^{2}-u^{2}\right) \\
& +\mathcal{R}_{0 h h}^{*} \frac{1}{2} h^{2}+\mathcal{R}_{0 k k}^{*} \frac{1}{2}\left(k-k_{0}\right)^{2} \\
& +\mathcal{R}_{0 \alpha \alpha}^{*} \frac{1}{2}\left(\alpha-\alpha_{0}\right)^{2}+\mathcal{R}_{0 k \alpha}^{*}\left(k-k_{0}\right)\left(\alpha-\alpha_{0}\right) \\
& +\mathcal{R}_{1 k}^{*} k^{\prime}\left(k-k_{0}\right)+\mathcal{R}_{1 \alpha}^{*} k^{\prime}\left(\alpha-\alpha_{0}\right)+\mathcal{R}_{2 h}^{*} h^{\prime} h \\
& +\left[\mathcal{R}_{3 k}^{*} \frac{1}{2}\left(v^{2}+u^{2}\right)+\mathcal{R}_{4 k}^{*} \frac{1}{2}\left(v^{2}-u^{2}\right)\right]\left(k-k_{0}\right) \\
& +\left[\mathcal{R}_{3 \alpha}^{*} \frac{1}{2}\left(v^{2}+u^{2}\right)+\mathcal{R}_{4 \alpha}^{*} \frac{1}{2}\left(v^{2}-u^{2}\right)\right]\left(\alpha-\alpha_{0}\right) \\
& +\mathcal{R}_{5 h}^{*} v u h+\mathcal{R}_{3}^{*} \frac{1}{2}\left(v^{2}+u^{\prime 2}\right)+\mathcal{R}_{4}^{*} \frac{1}{2}\left(v^{\prime 2}-u^{2}\right) \\
& +\mathcal{R}_{6}^{*} \frac{1}{2}\left(k^{\prime 2}+h^{\prime 2}\right)+\mathcal{R}_{7}^{*} \frac{1}{2}\left(k^{\prime 2}-h^{\prime 2}\right) \\
& +\mathcal{R}_{9}^{*}\left[\frac{1}{24}\left(v^{4}+u^{4}\right)+\frac{1}{12} v^{2} u^{2}\right]+\mathcal{R}_{10}^{*} \frac{1}{24}\left(v^{4}-u^{4}\right) \\
& +\mathcal{R}_{12}^{*}\left[\frac{1}{24}\left(v^{4}+u^{4}\right)-\frac{3}{12} v^{2} u^{2}\right] \\
& +\left[\mathcal{R}_{14}^{*} \frac{1}{2}\left(v^{2}+u^{2}\right)+\mathcal{R}_{15}^{*} \frac{1}{2}\left(v^{2}-u^{2}\right)\right] k^{\prime} \\
& \left.+\mathcal{R}_{19}^{*} v u h^{\prime}+\mathcal{R}_{21}^{*} u u^{\prime}+\mathcal{R}_{22}^{*} v v^{\prime}\right]
\end{aligned}
$$

onde os coeficientes $\mathcal{R}_{i}^{*}$ são os mesmos definidos no Anexo I (seção 6.1). Esta expressão em variáveis regulares resulta muito útil na hora de calcular as derivadas da função perturbadora.

Um dos principais inconvenientes do desenvolvimento assimétrico é o fato de possuir uma parte estritamente planar, que é conseqüência de fazer-se a expansão em torno de inclinações nulas. Isto significa que ele preserva todas as características morfológicas do desenvolvimento planar entre as quais, encontra-se a chamada "curva de colisão", que corresponde aos pontos do espaço $(k, h)$ onde a posição do perturbado coincide com a do perturbador, fazendo com que a função perturbadora tenha uma singularidade em $\mathbb{R}^{n}$. A Fig. 3.1 mostra a posição das curvas de colisão das ressonâncias $2 / 1$ e $3 / 2^{4}$.

${ }^{4} \mathrm{O}$ calculo da curva de colisão para uma ressonância $(p+q) / p$ é simples. Seja $a_{\mathrm{c}}$ o semieixo crítico da ressonância, $r, v$ o raio vetor e a anomalia verdadeira:

$$
r=\frac{a_{\mathrm{c}}\left(1-e^{2}\right)}{1+e \cos v} ; \quad \frac{r}{a_{\mathrm{c}}} \cos v=\cos u-e,
$$

onde $e$ a excentricidade, $u$ a anomalia excêntrica. Sejam $a^{\prime}, \ell^{\prime}$ o semieixo e a anomalia média do 


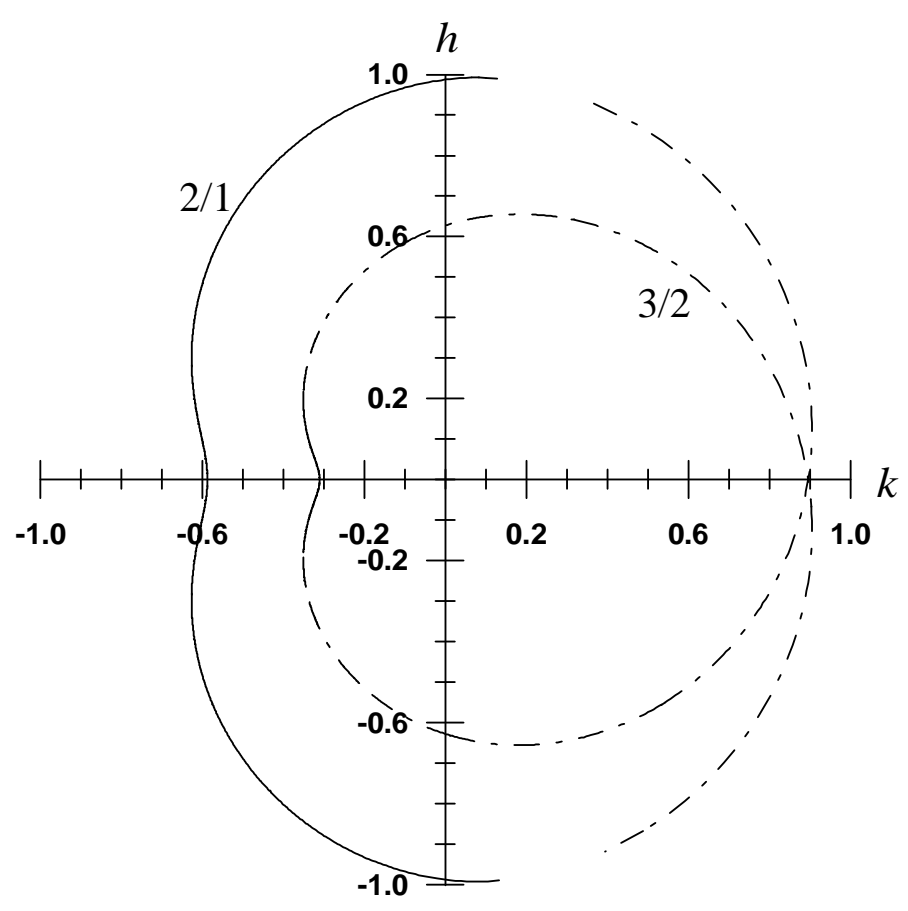

Figura 3.1: Curvas de colisão do problema planar.

É claro que num problema tridimensional real, onde as órbitas deixam de ser coplanares, a curva de colisão só aparece quando os objetos cruzam o nodo mútuo das órbitas. Porém, nosso desenvolvimento, mesmo sendo não planar, não consegue reproduzir este fato. Portanto, na hora da aplicação a um problema concreto, devemos levar em conta isto e estudar só problemas onde possamos garantir que os objetos manter-seão longe da curva de colisão. No caso das ressonâncias $2 / 1$ e $3 / 2$ isto pode ser garantido para amplitudes moderadas de oscilação do argumento ressonante $\left(|\sigma|<60^{\circ} \sim 90^{\circ}\right)$ e excentricidades moderadas ( $e \lesssim 0.5$ ), como podemos apreciar na Fig. 3.1.

Uma outra limitação do desenvolvimento assimétrico é que ele só é válido para perturbador, cuja órbita supomos como sendo circular. As condições para existir colisão são $r=a^{\prime} \mathrm{e}$ $v=\ell^{\prime}$, ou então, após alguns cálculos elementares

$$
\begin{aligned}
e \cos u & =1-\frac{a^{\prime}}{a_{\mathrm{c}}} \\
\sigma & =\frac{p+q}{q} v-\frac{p}{q}(u-e \sin u) .
\end{aligned}
$$

Assim, para um dado valor de $e$, obtemos $u$ com a primeira equação e $\sigma$ com a segunda, levando em conta que

$$
\tan \frac{v}{2}=\sqrt{\frac{1+e}{1-e}} \tan \frac{u}{2}
$$

Os valores de $k, h$ correspondentes à colisão são imediatos. 
inclinações moderadas. A hipótese $\sin I / 2 \sim \mathcal{O}\left(\sqrt{e^{\prime}}\right)$ significa que a validade do desenvolvimento não vai além de $I \sim 30^{\circ}$ (cf. fig. 3 no Anexo I).

\subsection{O mapeamento}

Uma vez que escolhemos o modelo, o passo seguinte é escolher o esquema simplético que vamos a utilizar para montar as equações do mapeamento. Em nosso caso, a função perturbadora depende dos ângulos e das ações, como é simples verificar das Eqs. (3.14) e (3.24). Portanto, o formalismo explícito em séries de Lie ou o mapeamento "leap-frog" resultam inadequados, já que as equações ficam expressadas em termos de séries infinitas. Contrariamente, o esquema implícito de primeira ordem é mais simples de implementar, e o único inconveniente está relacionado à solução simultânea das equações implícitas nas ações.

Começamos incorporando o desenvolvimento assimétrico da Eq. (3.24) na Hamiltoniana da Eq. (3.9). Chamando $\theta_{i}$ aos ângulos (Eqs. 3.3) e $J_{i}$ às ações (Eqs. 3.12), montamos a função geratriz

$$
S\left(\boldsymbol{\theta}^{n}, \mathbf{J}^{n+1}\right)=\sum_{i=1}^{3} \theta_{i}^{n} J_{i}^{n+1}+\tau \mathcal{H}^{*}\left(\boldsymbol{\theta}^{n}, \mathbf{J}^{n+1}\right),
$$

e o mapeamento fica definido como a transformação canônica

$$
\left(\boldsymbol{\theta}^{n}, \mathbf{J}^{n}\right) \rightarrow\left(\boldsymbol{\theta}^{n+1}, \mathbf{J}^{n+1}\right)
$$

através das equações implícitas

$$
\begin{aligned}
J_{i}^{n+1} & =J_{i}^{n}-\tau \frac{\partial \mathcal{H}^{*}\left(\boldsymbol{\theta}^{n}, \mathbf{J}^{n+1}\right)}{\partial \theta_{i}^{n}} \\
\theta_{i}^{n+1} & =\theta_{i}^{n}+\tau \frac{\partial \mathcal{H}^{*}\left(\theta^{n}, \mathbf{J}^{n+1}\right)}{\partial J_{i}^{n+1}}, \quad i=1,2,3 .
\end{aligned}
$$

Em função do que comentamos no Capítulo 2, o passo $\tau$ é escolhido como sendo o período da variável sinódica $Q$, isto é

$$
\tau=\frac{2 \pi q}{n-n^{\prime}}
$$

(em particular $q=1)^{5}$. É claro que o conjunto de equações implícitas (Eqs. 3.27) deve ser resolvido antes que as equações explícitas (Eqs. 3.28). Para isto, utilizamos

\footnotetext{
${ }^{5}$ Isto representa, aproximadamente, 11.8 anos.
} 
o método de aproximações sucessivas, tomando como valor inicial $\mathbf{J}^{n}$ e iterando as equações (3.27). Observou-se através de diferentes provas que o valor de $\mathbf{J}^{n+1}$ converge rapidamente; portanto, 10 ou 12 iterações são suficientes para conseguir uma precisão menor que $10^{-10}$ no valor final de $\mathbf{J}^{n+1}$.

No caso particular em que a Hamiltoniana depende explicitamente do tempo, o mapeamento pode ser estendido adicionando duas equações a mais. Em particular, podemos considerar o tempo $t$ como sendo um ângulo que possui associada uma certa ação $\mathcal{T}$. Então, definimos a nova Hamiltoniana $\mathcal{H}^{\prime}=\mathcal{H}^{*}+\mathcal{T}$ e obtemos as equações

$$
\begin{aligned}
\mathcal{T}^{n+1} & =\mathcal{T}^{n}-\tau \frac{\partial \mathcal{H}^{*}\left(t^{n}, \boldsymbol{\theta}^{n}, \mathbf{J}^{n+1}\right)}{\partial t^{n}} \\
t^{n+1} & =t^{n}+\tau .
\end{aligned}
$$

A primeira desta equações não fornece informação imprescindível para o mapeamento e pode ser ignorada ${ }^{6}$. A segunda equação é trivial e só devemos levar em conta que as equações (3.27) e (3.28) devem ser avaliadas a cada passo em $t^{n}$.

\subsection{As derivadas da Hamiltoniana}

Um último problema é calcular as derivadas da Hamiltoniana com respeito a $\psi, \sigma, \sigma_{z}$, $P, J, J_{z}$, que aparecem nos segundos membros das equações do mapeamento.

Por um lado, as derivadas da parte não perturbada da Hamiltoniana média são triviais. A partir das Eqs. (3.9) e (3.10), e chamando $r=\frac{p}{q}$, obtemos

$$
\frac{\partial \mathcal{H}_{0}}{\partial J_{i}}=-r \frac{\mu^{2}}{L^{3}}+(r+1) n^{\prime}
$$

onde utilizamos $J_{i}$ para designar genericamente às variáveis $P, J, J_{z}$.

Por outro lado, como o desenvolvimento assimétrico está escrito em função de um conjunto de variáveis que não são as variáveis ângulo e ação do mapeamento, as derivadas devem ser calculadas aplicando a regra da cadeia. Assim, obtemos

$$
\begin{aligned}
& \frac{\partial R}{\partial \psi}=\frac{\partial R}{\partial k^{\prime}} \frac{d k^{\prime}}{d \psi}+\frac{\partial R}{\partial h^{\prime}} \frac{d h^{\prime}}{d \psi}+\frac{\partial R}{\partial v^{\prime}} \frac{d v^{\prime}}{d \psi}+\frac{\partial R}{\partial u^{\prime}} \frac{d u^{\prime}}{d \psi} \\
& \frac{\partial R}{\partial \sigma}=\frac{\partial R}{\partial k} \frac{d k}{d \sigma}+\frac{\partial R}{\partial h} \frac{d h}{d \sigma}
\end{aligned}
$$

\footnotetext{
${ }^{6}$ Ela diz basicamente que a variação $\mathcal{T}^{n+1}-\mathcal{T}^{n}$ após um passo $\tau$ é aproximadamente igual à variação $\mathcal{H}\left(t^{n+1}\right)-\mathcal{H}\left(t^{n}\right)$. Portanto, o mapeamento preserva aproximadamente a hamiltoniana $\mathcal{H}^{\prime}=0$.
} 


$$
\begin{aligned}
\frac{\partial R}{\partial \sigma_{z}} & =\frac{\partial R}{\partial v} \frac{d v}{d \sigma_{z}}+\frac{\partial R}{\partial u} \frac{d u}{d \sigma_{z}} \\
\frac{\partial R}{\partial J_{i}} & =\frac{\partial R}{\partial \alpha} \frac{d \alpha}{d J_{i}}+\frac{\partial R}{\partial k} \frac{d k}{d J_{i}}+\frac{\partial R}{\partial h} \frac{d h}{d J_{i}}+\frac{\partial R}{\partial v} \frac{d v}{d J_{i}}+\frac{\partial R}{\partial u} \frac{d u}{d J_{i}}
\end{aligned}
$$

Apresentaremos a seguir as expressões explícitas destas derivadas.

\subsubsection{Derivadas do desenvolvimento assimétrico médio}

A partir da expressão Eq. (3.24), e suprimindo o uso do símbolo $\left(^{*}\right)$ para representar a média, as derivadas do desenvolvimento assimétrico resultam ser:

$$
\begin{aligned}
\frac{\partial R}{\partial \alpha}=\frac{1}{a^{\prime}}[ & \mathcal{R}_{0 \alpha}+\mathcal{R}_{0 k \alpha}\left(k-k_{0}\right)+\mathcal{R}_{0 \alpha \alpha}\left(\alpha-\alpha_{0}\right) \\
& \left.+\mathcal{R}_{1 \alpha} k^{\prime}+\left(\mathcal{R}_{3 \alpha}+\mathcal{R}_{4 \alpha}\right) \frac{1}{2} v^{2}+\left(\mathcal{R}_{3 \alpha}-\mathcal{R}_{4 \alpha}\right) \frac{1}{2} u^{2}\right]
\end{aligned}
$$

$$
\begin{aligned}
\frac{\partial R}{\partial k}=\frac{1}{a^{\prime}}[ & \mathcal{R}_{0 k}+\mathcal{R}_{0 k \alpha}\left(\alpha-\alpha_{0}\right)+\mathcal{R}_{0 k k}\left(k-k_{0}\right) \\
& \left.+\mathcal{R}_{1 k} k^{\prime}+\left(\mathcal{R}_{3 k}+\mathcal{R}_{4 k}\right) \frac{1}{2} v^{2}+\left(\mathcal{R}_{3 k}-\mathcal{R}_{4 k}\right) \frac{1}{2} u^{2}\right]
\end{aligned}
$$

$$
\begin{aligned}
\frac{\partial R}{\partial h}=\frac{1}{a^{\prime}}[ & \left.\mathcal{R}_{0 h h} h+\mathcal{R}_{2 h} h^{\prime}+\mathcal{R}_{5 h} v u\right] \\
\frac{\partial R}{\partial v}=\frac{1}{a^{\prime}}[ & \left(\mathcal{R}_{3}+\mathcal{R}_{4}\right) v+\mathcal{R}_{5 h} u h+\left(\mathcal{R}_{3 k}+\mathcal{R}_{4 k}\right) v\left(k-k_{0}\right) \\
& +\left(\mathcal{R}_{3 \alpha}+\mathcal{R}_{4 \alpha}\right) v\left(\alpha-\alpha_{0}\right)+\left(\mathcal{R}_{9}+\mathcal{R}_{10}+\mathcal{R}_{12}\right) \frac{1}{6} v^{3} \\
& +\left(\mathcal{R}_{9}-3 \mathcal{R}_{12}\right) \frac{1}{6} v u^{2}+\left(\mathcal{R}_{14}+\mathcal{R}_{15}\right) v k^{\prime} \\
& \left.+\mathcal{R}_{19} u h^{\prime}+\mathcal{R}_{22} v^{\prime}\right]
\end{aligned}
$$

$$
\begin{aligned}
\frac{\partial R}{\partial u}=\frac{1}{a^{\prime}}[ & \left(\mathcal{R}_{3}-\mathcal{R}_{4}\right) u+\mathcal{R}_{5 h} v h+\left(\mathcal{R}_{3 k}-\mathcal{R}_{4 k}\right) u\left(k-k_{0}\right) \\
& +\left(\mathcal{R}_{3 \alpha}-\mathcal{R}_{4 \alpha}\right) u\left(\alpha-\alpha_{0}\right)+\left(\mathcal{R}_{9}-\mathcal{R}_{10}+\mathcal{R}_{12}\right) \frac{1}{6} u^{3} \\
& +\left(\mathcal{R}_{9}-3 \mathcal{R}_{12}\right) \frac{1}{6} v^{2} u+\left(\mathcal{R}_{14}-\mathcal{R}_{15}\right) u k^{\prime} \\
& \left.+\mathcal{R}_{19} v h^{\prime}+\mathcal{R}_{21} u^{\prime}\right]
\end{aligned}
$$

$$
\begin{aligned}
\frac{\partial R}{\partial k^{\prime}}=\frac{1}{a^{\prime}}[ & \mathcal{R}_{1}+\mathcal{R}_{1 k}\left(k-k_{0}\right)+\mathcal{R}_{1 \alpha}\left(\alpha-\alpha_{0}\right)+\left(\mathcal{R}_{6}+\mathcal{R}_{7}\right) k^{\prime} \\
& \left.+\left(\mathcal{R}_{14}+\mathcal{R}_{15}\right) \frac{1}{2} v^{2}+\left(\mathcal{R}_{14}-\mathcal{R}_{15}\right) \frac{1}{2} u^{2}\right]
\end{aligned}
$$




$$
\begin{aligned}
\frac{\partial R}{\partial h^{\prime}} & =\frac{1}{a^{\prime}}\left[\mathcal{R}_{2 h} h+\left(\mathcal{R}_{6}-\mathcal{R}_{7}\right) h^{\prime}+\mathcal{R}_{19} v u\right] \\
\frac{\partial R}{\partial v^{\prime}} & =\frac{1}{a^{\prime}}\left[\left(\mathcal{R}_{3}+\mathcal{R}_{4}\right) v^{\prime}+\mathcal{R}_{22} v\right] \\
\frac{\partial R}{\partial u^{\prime}} & =\frac{1}{a^{\prime}}\left[\left(\mathcal{R}_{3}-\mathcal{R}_{4}\right) u^{\prime}+\mathcal{R}_{21} u\right]
\end{aligned}
$$

\subsubsection{Derivadas das variáveis do desenvolvimento}

As relações entre as variáveis $\alpha, k, h, v, u, \ldots$ etc., e as variáveis ação e ângulo do problema ressonante, são dadas pelas Eqs. (3.10), (3.13), (3.14), (3.15) e (3.23). Após alguns cálculos simples, obtemos as seguintes expressões para as derivadas:

Derivadas de $B$ e $D$ :

$$
\begin{aligned}
& \frac{d B}{d P}=r \frac{L-J}{2 B L^{3}}=r\left(\frac{B}{L}-\frac{1}{2 B L^{2}}\right)=\frac{d B}{d J_{z}} \\
& \frac{d B}{d J}=\left(\frac{d B}{d P}-\frac{1}{4 B L^{2}}\right) \\
& \frac{d D}{d P}=\frac{r}{8 D(L-J)^{2}}=2 r D^{3}=\frac{d D}{d J_{z}} \\
& \frac{d D}{d J}=\left(\frac{d D}{d P}+2 D^{3}\right) .
\end{aligned}
$$

Derivadas de $\alpha$ :

$$
\frac{d \alpha}{d P}=-r \frac{2 L}{\mu a^{\prime}}=\frac{d \alpha}{d J}=\frac{d \alpha}{d J_{z}}
$$

Derivadas de $k$ e $h$ :

$$
\begin{aligned}
\frac{d k}{d \sigma}+i \frac{d h}{d \sigma} & =i k-h \\
\frac{d k}{d P}+i \frac{d h}{d P} & =\frac{d B}{d P} \sqrt{2 J} \exp i \sigma=\frac{d k}{d J_{z}}+i \frac{d h}{d J_{z}} \\
\frac{d k}{d J}+i \frac{d h}{d J} & =\left(\frac{d B}{d J} \sqrt{2 J}+\frac{B}{\sqrt{2 J}}\right) \exp i \sigma .
\end{aligned}
$$

Derivadas de $v$ e $u$ :

$$
\begin{aligned}
\frac{d v}{d \sigma_{z}}+i \frac{d u}{d \sigma_{z}} & =i v-u \\
\frac{d v}{d P}+i \frac{d u}{d P} & =\frac{d D}{d P} \sqrt{2 J_{z}} \exp i \sigma_{z}
\end{aligned}
$$




$$
\begin{aligned}
\frac{d v}{d J}+i \frac{d u}{d J} & =\frac{d D}{d J} \sqrt{2 J_{z}} \exp i \sigma_{z} \\
\frac{d v}{d J_{z}}+i \frac{d u}{d J_{z}} & =\left(\frac{d D}{d J_{z}} \sqrt{2 J_{z}}+\frac{D}{\sqrt{2 J_{z}}}\right) \exp i \sigma_{z} .
\end{aligned}
$$

Derivadas de $k^{\prime}, h^{\prime}, v^{\prime}$ e $u^{\prime}$ :

$$
\begin{aligned}
& \frac{d k^{\prime}}{d \psi}+i \frac{d h^{\prime}}{d \psi}=i k^{\prime}-h^{\prime} \\
& \frac{d v^{\prime}}{d \psi}+i \frac{d u^{\prime}}{d \psi}=i v^{\prime}-u^{\prime}
\end{aligned}
$$

É importante destacar que algumas destas derivadas (Eqs. 3.45 e 3.46) tornam-se singulares quando $e=0$ ou $I=0$, isto é quando $J=0$ ou $J_{z}=0$. Esta singularidade está vinculada ao fato de que o próprio conjunto de variáveis ação e ângulo é singular na origem ( $\sigma$ e $\sigma_{z}$ não estão definidos). Portanto, uma forma de evitar esta singularidade seria trabalhar com as variáveis regulares

$$
\begin{aligned}
K+i H & =\sqrt{2 J} \exp i \sigma \\
V+i U & =\sqrt{2 J_{z}} \exp i \sigma_{z} .
\end{aligned}
$$

No entanto, como já foi discutido, o esquema simplético que utilizamos para construir o mapeamento exige o uso de variáveis ação e ângulo. Assim, a única forma em que podemos evitar a singularidade das equações é impedir que $J$ ou $J_{z}$ possam se anular em algum momento da integração. Para isto, podemos monitorar a cada passo do mapeamento os valores de $J$ e $J_{z}$ e interromper a integração quando

$$
J<J_{\min }, \quad J_{z}<J_{z \min },
$$

onde estes valores mínimos são escolhidos "ad-hoc" segundo algum critério razoável.

Isto conclui com a obtenção das derivadas de $R^{*}$ e com a definição de nosso modelo. No próximo capítulo, discutiremos a forma de melhorar o modelo para que seja mais realístico, através da inclusão de variações temporais nos elementos orbitais do corpo perturbador. 


\section{VARIAÇÕES DE LONGO PERÍODO DA ÓRBITA DO PERTURBADOR}

A inclusão de variações na órbita do corpo perturbador não só fornece um modelo mais realista do problema, mas também acrescenta graus de liberdade ao problema, fato que contribui para gerar novos fenômenos de difusão caótica na ressonância e para acelerar os que, eventualmente, já existem. Há dois tipos de variações temporais que afetam significativamente à órbita de Júpiter: as variações seculares devidas aos planetas externos e as variações de curto período devidas à comensurabilidade 5/2 entre os movimentos médios de Júpiter e Saturno, chamada comumente de grande desigualdade. Neste capítulo apresentaremos as perturbações que são consideradas no trabalho e explicaremos por que são consideradas.

\subsection{Teorias seculares sintéticas}

Sabemos a partir da teoria linear clássica que, num sistema de $N$-corpos, as variações seculares das órbitas dos corpos envolvidos podem ser expressadas como uma superposição de harmônicos da forma

$$
\begin{aligned}
e_{j} \exp i \varpi_{j} & =\sum_{l=1}^{N} G_{j l} \exp \left[i\left(g_{l} t+\gamma_{l}\right)\right] \\
\sin \frac{I_{j}}{2} \exp i \Omega_{j} & =\sum_{l=1}^{N} S_{j l} \exp \left[i\left(s_{l} t+\sigma_{l}\right)\right], \quad(j=1, \ldots, N)
\end{aligned}
$$


onde as amplitudes $G_{j l}, S_{j l}$ e as freqüências $g_{l}, s_{l}$ determinam os modos de oscilação própria do sistema.

Uma forma de se obter uma teoria secular deste tipo consiste em sintetizar-la a partir de simulações numéricas, ajustando os parâmetros do modelo a um conjunto de dados "observacionais". Para fazer isto, foi criado o programa LONGSTOP (Milani et al., 1987; Carpino et al., 1987; Nobili et al., 1989) cuja idéia foi fazer uma integração numérica do sistema solar exterior (de Júpiter a Plutão) por 100 milhões de anos e utilizar esses resultados como a base para sintetizar uma teoria do movimento secular destes planetas.

O programa foi dividido em duas partes. A primeira (LONGSTOP 1A), consistiu numa integração por 9.3 milhões de anos, que visava experimentar a metodologia que seria aplicada, para conhecer as limitações do processo e as possíveis melhoras. A segunda parte (LONGSTOP 1B) foi propriamente uma integração por 100 milhões de anos (50 milhões para a frente e 50 milhões para trás) que visava levar em conta também os efeitos dos planetas internos e correções relativistas. O processamento dos resultados envolveu três etapas principais: primeiro, aplicar à saída dos dados uma técnica de filtragem digital de freqüências, visando eliminar aquelas freqüências de curto período. Em segundo lugar, aplicar a análise de Fourier aos resultados filtrados de forma a obter as linhas espectrais, junto com as amplitudes e as fases, devidas aos termos de longo período. Finalmente, a terceira etapa consistiu em ajustar os parâmetros de um dado modelo aos resultados obtidos, para descrever a evolução secular dos semieixos e das variáveis $k, h$ e $v, u$. O modelo que foi ajustado possui a forma

$$
\sum_{j} M_{j} \exp i\left[\sum_{i=1}^{n} m_{i j}\left(\mu_{i} t+\nu_{i}\right)\right],
$$

onde os parâmetros inteiros $m_{i j}$ e a quantidade $n$ destes parâmetros que aparecem em cada argumento são propostos "a priori" a partir de considerações teóricas, e os restantes parâmetros $M_{j}, \mu_{i}$ e $\nu_{i}$ (amplitudes, freqüências e fases respectivamente) são determinados através de um ajuste por mínimos quadrados a partir dos dados numéricos.

O resultado deste projeto é um conjunto de tabelas onde são listadas as principais combinações de freqüências dos diferentes planetas e as amplitudes desses termos para cada planeta, juntamente com a fases iniciais (Nobili et al., 1989). 


\begin{tabular}{|c|c|c|c|c|}
\hline$l$ & Freqüência & Fase & Amplitude & Amplitude \\
\hline & $g_{l}[" / \mathrm{ano}]$ & $\gamma_{l}\left[{ }^{\circ}\right]$ & Júpiter & Saturno \\
\hline 5 & 4.25749319 & 27.0005 & $4.4187210^{-2}$ & $3.2958310^{-2}$ \\
\hline 6 & 28.24552984 & 124.1994 & $-1.5700210^{-2}$ & $4.8209310^{-2}$ \\
\hline 7 & 3.08675577 & 117.0516 & $1.813910^{-3}$ & $1.511310^{-3}$ \\
\hline 8 & 0.67255084 & 70.7508 & $5.8010^{-5}$ & $5.7510^{-5}$ \\
\hline$l$ & $s_{l}[" /$ ano $]$ & $\sigma_{l}\left[^{\circ}\right]$ & Júpiter & \\
\hline 5 & -0.01000400 & 182.7100 & $1.71110^{-5}$ & - \\
\hline 6 & -26.34496354 & 303.9699 & $3.1532910^{-3}$ & - \\
\hline 7 & -2.99266093 & 316.7946 & $-4.847810^{-4}$ & - \\
\hline 8 & -0.69251368 & 200.3717 & $-5.844810^{-4}$ & - \\
\hline
\end{tabular}

Tabela 4.1: Freqüências fundamentais, fases e amplitudes da variação secular (JD 2440400.5). Por convenção, o subíndice $l=5$ corresponde a Júpiter, $l=6$ a Saturno, etc.

\subsection{Perturbações seculares consideradas no trabalho}

As variações seculares nos elementos orbitais de Júpiter consideradas no trabalho foram tiradas da teoria secular sintética mencionada acima. As amplitudes $G_{j l}, S_{j l}$, as freqüências $g_{l}, s_{l}$ e as fases iniciais $\gamma_{l}, \sigma_{l}$ utilizadas são apresentadas nas Tabelas 4.1 e 4.2. Em particular, só levamos em conta aqueles termos com amplitude $G_{j l} \geq 10^{-4} \mathrm{e}$ $S_{j l} \geq 10^{-5}$, respectivamente. No entanto, percebamos que as freqüências $g_{8}$ e $2 g_{5}-g_{6}$ possuem amplitudes ainda menores; mesmo assim, elas são consideradas já que, $g_{8}$ é uma das freqüências fundamentais do sistema (a do periélio de Netuno), e $2 g_{5}-g_{6}$ é a freqüência retrógrada de maior período do periélio de Júpiter, que ocorre na grande desigualdade do sistema Júpiter-Saturno. Os valores apresentados são dados para JD 2440400.5. Um detalhe interessante é que a freqüência $s_{5}$, correspondente ao movimento do nodo de Júpiter, não é nula já que o modelo considera as perturbações devidas aos planetas interiores ${ }^{1}$. Nas Tabelas 4.1 e 4.2, apresentamos também as amplitudes da variação na excentricidade e periélio de Saturno, que utilizaremos mais adiante.

\footnotetext{
${ }^{1}$ Se as perturbações dos planetas internos não fossem consideradas, a freqüência $s_{5}$ seria nula devido à conservação do momento angular total do sistema dos planetas externos
} 


\begin{tabular}{|c|c|c|c|}
\hline & Período [anos] & Amplitude & Amplitude \\
\hline & & Júpiter & Saturno \\
\hline \multicolumn{3}{|l|}{ Termos em $e_{j} \exp i \varpi_{j}$} & $5.98210^{-4}$ \\
\hline$-g_{5}+g_{6}+g_{7}$ & $4.786740310^{4}$ & $1.98210^{-4}$ & $-6.05210^{-4}$ \\
\hline$-g_{5}+2 g_{6}$ & $2.481163110^{4}$ & $-5.73510^{-4}$ & $1.919410^{-3}$ \\
\hline $2 g_{5}-g_{6}$ & $-6.568496210^{4}$ & $1.4210^{-5}$ & $-1.41410^{-4}$ \\
\hline$g_{5}-s_{6}+s_{7}$ & $4.693986210^{4}$ & $1.10410^{-4}$ & $-3.36810^{-4}$ \\
\hline$-g_{5}+2 g_{6}+s_{6}-s_{7}$ & $4.487338210^{4}$ & $-1.22610^{-4}$ & $3.79210^{-4}$ \\
\hline \multicolumn{4}{|c|}{ Termos em $\sin \left(I_{j} / 2\right) \exp i \Omega_{j}$} \\
\hline$s_{6}-g_{5}+g_{6}$ & $-5.498692210^{5}$ & $1.78910^{-5}$ & - \\
\hline$s_{7}+g_{5}-g_{6}$ & $-4.803433510^{4}$ & $2.38510^{-5}$ & - \\
\hline$s_{7}+g_{5}-g_{7}$ & $-7.113357210^{5}$ & $-1.16610^{-5}$ & - \\
\hline$-s_{6}+2 g_{6}$ & $1.564536610^{4}$ & $1.42810^{-5}$ & - \\
\hline
\end{tabular}

Tabela 4.2: Termos mistos da variação secular.

Digamos para finalizar, que as variações seculares no semieixo podem ser desprezadas já que elas são por volta de seis ordens de magnitude menores que nos restantes elementos (Milani et al., 1987). Por sua vez, as variações seculares na longitude média da época, $\varepsilon$, são de 20 a 100 vezes menores que as respectivas variações em $e$ e $\sin I / 2$, e portanto também podemos desprezá-las

\subsection{Perturbações críticas de curto período}

Até agora só falamos das variações seculares, sem levar em conta que certas variações periódicas de curto período podem tornar-se críticas em função da proximidade a uma comensurabilidade de movimentos médios. Esta situação é responsável pela aparição de termos na função perturbadora cujo período é muito maior que o período dos termos não críticos, porém bem menor que o período dos termos seculares. Ainda mais, a ocorrência de um destes termos críticos faz com que nas soluções de primeira ordem das equações de Lagrange, apareça um pequeno divisor no coeficiente que acompanha a esse argumento crítico. Assim, estes termos podem ter também um efeito significativo no comportamento ao longo prazo do sistema.

No caso Júpiter-Saturno, a já mencionada "grande desigualdade" faz com que o 


\begin{tabular}{ccc}
\hline \hline Freqüência & Fase & Período \\
\hline$-1467.2852^{\prime \prime} /$ ano & $178.3158^{\circ}$ & 883.26 anos \\
& & \\
\hline \hline
\end{tabular}

Tabela 4.3: Freqüência, fase inicial e período do argumento $\varphi=2 \lambda_{\mathrm{J}}-5 \lambda_{\mathrm{S}}$ (JD 2451545).

argumento $2 \lambda_{\mathrm{J}}-5 \lambda_{\mathrm{S}}$ apresente uma circulação lenta, com um período de aproximadamente 880 anos. Nesse caso, a presença do pequeno divisor $2 n_{\mathrm{J}}-5 n_{\mathrm{S}}$ não chega a afetar as propriedades de convergência das soluções das equações de Lagrange e o tratamento clássico do problema continua sendo válido. Para obter a expressão analítica destas variações de curto período, começamos considerando só os principais termos críticos da função perturbadora devidos a esta comensurabilidade, e escrevemos

$$
\begin{gathered}
R_{\text {crit }}=m_{\mathrm{S}}\left[f_{1} e_{\mathrm{J}} e_{\mathrm{S}}^{2} \cos \left(\varphi+\varpi_{\mathrm{J}}+2 \varpi_{\mathrm{S}}\right)+f_{2} e_{\mathrm{J}}^{2} e_{\mathrm{S}} \cos \left(\varphi+2 \varpi_{\mathrm{J}}+\varpi_{\mathrm{S}}\right)\right. \\
\left.+f_{3} e_{\mathrm{J}}^{3} \cos \left(\varphi+3 \varpi_{\mathrm{J}}\right)+f_{4} e_{\mathrm{S}}^{3} \cos \left(\varphi+3 \varpi_{\mathrm{S}}\right)+\cdots\right]
\end{gathered}
$$

onde $m_{\mathrm{S}}$ é a massa de Saturno, $f_{k}$ são coeficientes que dependem de $a_{\mathrm{J}} / a_{\mathrm{S}}$, e

$$
\varphi=2 \lambda_{\mathrm{J}}-5 \lambda_{\mathrm{S}}=\nu_{\varphi} t+\varphi_{0}
$$

Os valores da freqüência $\nu_{\varphi}$ e da fase $\varphi_{0}$ para J2000.0 aparecem na Tabela 4.3.

A seguir, escrevemos as equações de Lagrange para a excentricidade e o periélio:

$$
\frac{d e_{\mathrm{J}}}{d t}=-\frac{\sqrt{1-e_{\mathrm{J}}^{2}}}{n_{\mathrm{J}} a_{\mathrm{J}}^{2} e_{\mathrm{J}}} \frac{\partial R_{\mathrm{crit}}}{\partial \varpi_{\mathrm{J}}}, \quad \frac{d \varpi_{\mathrm{J}}}{d t}=\frac{\sqrt{1-e_{\mathrm{J}}^{2}}}{n_{\mathrm{J}} a_{\mathrm{J}}^{2} e_{\mathrm{J}}} \frac{\partial R_{\text {crit }}}{\partial e_{\mathrm{J}}} .
$$

A integração aproximada destas equações fornece uma solução que possui a forma

$$
\begin{aligned}
\delta e_{\mathrm{J}}= & {\left[A_{1} e_{\mathrm{S}}^{2} \cos \left(\varphi+\varpi_{\mathrm{J}}+2 \varpi_{\mathrm{S}}\right)+A_{2} e_{\mathrm{J}} e_{\mathrm{S}} \cos \left(\varphi+2 \varpi_{\mathrm{J}}+\varpi_{\mathrm{S}}\right)\right.} \\
& \left.+A_{3} e_{\mathrm{J}}^{2} \cos \left(\varphi+3 \varpi_{\mathrm{J}}\right)\right] \\
e_{\mathrm{J}} \delta \varpi_{\mathrm{J}}= & {\left[B_{1} e_{\mathrm{S}}^{2} \sin \left(\varphi+\varpi_{\mathrm{J}}+2 \varpi_{\mathrm{S}}\right)+B_{2} e_{\mathrm{J}} e_{\mathrm{S}} \sin \left(\varphi+2 \varpi_{\mathrm{J}}+\varpi_{\mathrm{S}}\right)\right.} \\
& \left.+B_{3} e_{\mathrm{J}}^{2} \sin \left(\varphi+3 \varpi_{\mathrm{J}}\right)\right]
\end{aligned}
$$

Os coeficientes $A_{i}, B_{i}$ são apresentados na Tabela 4.4, e foram fornecidos por Simon (comunicação pessoal). 


\begin{tabular}{cccc}
\hline \hline$i$ & $A_{i}$ & $B_{i}$ & $C_{i}$ \\
\hline & & & \\
1 & $-22418.797^{\prime \prime}$ & $22260.089^{\prime \prime}$ & $9848888.254^{\prime \prime}$ \\
2 & $26745.689^{\prime \prime}$ & $-26646.324^{\prime \prime}$ & $-5862200.658^{\prime \prime}$ \\
3 & $-7782.128^{\prime \prime}$ & $7762.464^{\prime \prime}$ & $1133729.695^{\prime \prime}$ \\
4 & - & - & $-5434322.245^{\prime \prime}$ \\
& & & \\
\hline \hline
\end{tabular}

Tabela 4.4: Amplitudes das variações periódicas em $e_{\mathrm{J}}, \varpi_{\mathrm{J}}$ e $\lambda_{\mathrm{J}}$ devidas à comensurabilidade $5 / 2$.

As variações periódicas em inclinação e nodo $\left(\delta \sin \left(I_{\mathrm{J}} / 2\right)\right.$ e $\sin \left(I_{\mathrm{J}} / 2\right) \delta \Omega_{\mathrm{J}}$ respectivamente) devidas à comensurabilidade $5 / 2$ são 100 vezes menores que as variações em excentricidade e periélio (Simon e Bretagnon, 1975), e portanto podemos desprezalas. O mesmo acontece com as variações periódicas no semieixo $\left(\delta a_{\mathrm{J}} /\left\langle a_{\mathrm{J}}\right\rangle\right)$ que são umas 10 vezes menores (Michtchenko, comunicação pessoal). No entanto a variação em longitude média $\left(\delta \lambda_{\mathrm{J}}\right)$ é umas 10 vezes maior e portanto deve ser levada em conta.

Classicamente, costuma-se introduzir a longitude média escrevendo

$$
\lambda=\rho+\varepsilon
$$

onde $\rho=\int n d t$ e $\varepsilon$ é a longitude média da época. Logo, as equações de Lagrange são escritas na forma

$$
\frac{d^{2} \rho_{\mathrm{J}}}{d t^{2}}=-\frac{3}{a_{\mathrm{J}}^{2}} \frac{\partial R_{\text {crit }}}{\partial \lambda_{\mathrm{J}}}, \quad \frac{d \varepsilon_{\mathrm{J}}}{d t}=\frac{2}{n_{\mathrm{J}} a_{\mathrm{J}}} \frac{\partial R_{\text {crit }}}{\partial a_{\mathrm{J}}}
$$

e a integração aproximada fornece uma solução $\delta \lambda_{\mathrm{J}}=\delta \rho_{\mathrm{J}}+\delta \varepsilon_{\mathrm{J}}$, que podemos escrever como sendo

$$
\begin{gathered}
\delta \lambda_{\mathrm{J}}=\left[C_{1} e_{\mathrm{J}} e_{\mathrm{S}}^{2} \sin \left(\varphi+\varpi_{\mathrm{J}}+2 \varpi_{\mathrm{S}}\right)+C_{2} e_{\mathrm{J}}^{2} e_{\mathrm{S}} \sin \left(\varphi+2 \varpi_{\mathrm{J}}+\varpi_{\mathrm{S}}\right)\right. \\
\left.+C_{3} e_{\mathrm{J}}^{3} \sin \left(\varphi+3 \varpi_{\mathrm{J}}\right)+C_{4} e_{\mathrm{S}}^{3} \sin \left(\varphi+3 \varpi_{\mathrm{S}}\right)\right]
\end{gathered}
$$

Os coeficientes $C_{i}$ também foram fornecidos por Simon e aparecem na Tabela 4.4.

Para poder utilizar as Eqs (4.6) e (4.9) precisamos conhecer os valores de $e_{\mathrm{J}}, e_{\mathrm{S}}$ e de $\varpi_{\mathrm{J}}, \varpi_{\mathrm{S}}$. Estes valores para J2000.0 são apresentados na Tabela 4.5; no entanto, eles podem ser calculados para um instante de tempo qualquer a partir das Eqs. (4.1) da teoria secular.

Um detalhe importante é que os coeficientes $A_{i}, B_{i}$ e $C_{i}$ contém embutido o pequeno divisor $\nu_{\varphi}=2 n_{\mathrm{J}}-5 n_{\mathrm{S}}$ (Tabela 4.3). No caso das Eqs. (4.6) os coeficientes são inversa- 


\begin{tabular}{rrr}
\hline \hline & \multicolumn{1}{c}{ Júpiter } & \multicolumn{1}{c}{ Saturno } \\
\hline & & \\
$e$ & 0.048498 & 0.055548 \\
$\varpi$ & $14.331206^{\circ}$ & $93.057224^{\circ}$ \\
& & \\
\hline \hline
\end{tabular}

Tabela 4.5: Excentricidade e periélio médios em J2000.0.

mente proporcionais ao pequeno divisor; podemos introduzir então novos coeficientes $A_{i}^{\prime}, B_{i}^{\prime}$ tais que

$$
A_{i}=\frac{A_{i}^{\prime}}{\nu_{\varphi}}, \quad B_{i}=\frac{B_{i}^{\prime}}{\nu_{\varphi}}
$$

No entanto, no caso da Eq. (4.9) esta dependência é um pouco mais complicada, devido a que os coeficientes da expressão para $\delta \rho$ são proporcionais a $\nu_{\varphi}^{-2}$ (a equação de Lagrange para $\rho$ é de segunda ordem), enquanto que os coeficientes de $\delta \varepsilon$ são proporcionais a $\nu_{\varphi}^{-1}$. Porém, é simples ver que $\nu_{\varphi}^{-2}$ domina o comportamento destes coeficientes já que é umas 200 vezes maior que $\nu_{\varphi}^{-1}$. Assim, podemos introduzir novos coeficientes $C_{i}^{\prime}$ e escrever em forma aproximada

$$
C_{i} \simeq \frac{C_{i}^{\prime}}{\nu_{\varphi}^{2}}
$$

Com isto, obtemos expressões analíticas muito bem aproximadas das principais variações periódicas devidas à grande desigualdade.

\subsubsection{Precisão das expressões para as variações de curto período}

A precisão das expressões para $\delta e_{\mathrm{J}}, \delta \varpi_{\mathrm{J}}$ e $\delta \lambda_{\mathrm{J}}$ pode ser avaliada fazendo-se uma comparação com as perturbações devidas à grande desigualdade que são fornecidas por teorias do movimento planetário mais completas.

Substituindo os valores da Tabela 4.5 nas Eqs. (4.6) e (4.9) obtemos as seguintes expressões, válidas para um intervalo de tempo não maior do que $\sim 2000$ anos em torno de J2000.0:

$$
\begin{aligned}
\delta e_{\mathrm{J}} & =-72.972^{\prime \prime} \sin \varphi+13.547^{\prime \prime} \cos \varphi \\
e_{\mathrm{J}} \delta \varpi_{\mathrm{J}} & =-13.263^{\prime \prime} \sin \varphi-72.605^{\prime \prime} \cos \varphi \\
\delta \lambda_{\mathrm{J}} & =-1032.172^{\prime \prime} \sin \varphi-158.631^{\prime \prime} \cos \varphi
\end{aligned}
$$

No entanto, utilizando uma teoria secular de segunda ordem completa, como por 
exemplo, Simon e Bretagnon $(1975,1984)$, as expressões anteriores são

$$
\begin{aligned}
\delta e_{\mathrm{J}} & =-73.13^{\prime \prime} \sin \varphi+17.30^{\prime \prime} \cos \varphi \\
e_{\mathrm{J}} \delta \varpi_{\mathrm{J}} & =-16.85^{\prime \prime} \sin \varphi-73.03^{\prime \prime} \cos \varphi \\
\delta \lambda_{\mathrm{J}} & =-1168.463^{\prime \prime} \sin \varphi-184.051^{\prime \prime} \cos \varphi
\end{aligned}
$$

Fazendo-se a comparação podemos ver que, em média, as Eqs. (4.6) e (4.9) representam às variações periódicas do perturbador devidas à grande desigualdade com um grau de aproximação da ordem de $10 \%$.

Não devemos esquecer que, além do argumento $\varphi$ já analisado, a grande desigualdade introduz também termos periódicos correspondentes a outras combinações das longitudes médias. Dentre elas, a mais importante é a combinação $\varphi^{\prime}=\lambda_{\mathrm{J}}-2 \lambda_{\mathrm{S}}$, cuja freqüência é $21264.41^{\prime \prime}$ /ano. As variações periódicas devidas a este argumento, segundo Simon e Bretagnon $(1975,1984)$, são

$$
\begin{aligned}
\delta e_{\mathrm{J}} & =-30.62^{\prime \prime} \sin \varphi^{\prime}+130.30^{\prime \prime} \cos \varphi^{\prime} \\
e_{\mathrm{J}} \delta \varpi_{\mathrm{J}} & =-129.22^{\prime \prime} \sin \varphi^{\prime}-29.87^{\prime \prime} \cos \varphi^{\prime} \\
\delta \lambda_{\mathrm{J}} & =127.164^{\prime \prime} \sin \varphi^{\prime}-18.612^{\prime \prime} \cos \varphi^{\prime}
\end{aligned}
$$

Percebamos que as amplitudes em $\delta e_{\mathrm{J}}$ e $\delta \varpi_{\mathrm{J}}$ são quase duas vezes maiores que no caso das Eqs. (4.13); por sua vez, a amplitude em $\delta \lambda_{\mathrm{J}}$ é superior a $100^{\prime \prime}$. No entanto, estes termos podem não ser levados em conta sob duas argumentações: por um lado, seu período é muito pequeno (apenas 60.95 anos) em comparação com o período do argumento $\varphi$, e por outro lado, experimentos feitos por Ferraz-Mello (1997) utilizando o mapeamento plano mostraram que a inclusão destes termos não afeta em nada os resultados finais.

\subsection{Comentários}

As Eqs. (4.1), e as Eqs. (4.6) e (4.9), junto com as definições dos coeficientes das Eqs. (4.10) e (4.11), constituem o conjunto de variações da órbita de Júpiter consideradas no trabalho. É importante destacar que o valor da freqüência $\nu_{\varphi}$ pode ser modificado "ad-hoc" sem que isto altere a forma das relações Eqs. (4.6) e (4.9). Assim, nosso modelo pode ser utilizado muito facilmente como laboratório para experimentar como influi o valor desta freqüência no comportamento dos objetos sob estudo. 
Dois detalhes devem ser levados em conta na hora da utilização destas fórmulas. O primeiro é que as expressões para $\delta e, \delta \varpi$ fornecidas nas Eqs. (4.6) só podem ser utilizadas se pudermos garantir que $e$ nunca vá anular-se (devido à forma das Eqs. 4.5). No caso de Júpiter, sabemos (a partir da teoria secular) que $e_{\mathrm{J}}>0.03$ sempre; portanto o uso das citadas expressões não oferece problema nenhum. No entanto, se a condição $e_{\mathrm{J}} \neq 0$ não pudesse ser garantida, as Eqs. (4.6) devem ser substituídas por suas análogas nas variáveis regulares $k, h$.

O segundo detalhe é que as fases iniciais da teoria secular (Eqs. 4.1) são dadas para uma data juliana diferente da data da fase inicial das variações periódicas (Tabela 4.3). A diferença entre as duas datas é 11145 dias ( 30.5 anos). A máxima variação secular nos elementos de Júpiter durante esse intervalo de tempo acontece na excentricidade, e não é maior que 3\%, o que fica dentro da precisão das Eqs. (4.6) como já foi discutido. Portanto, aos efeitos práticos, podemos desprezar a diferença nas datas iniciais, e utilizar como origem comum de todas as expressões uma data genérica $t=0$.

\subsection{Incorporação das variações ao mapeamento}

A inclusão no mapeamento das variações na órbita de Júpiter é feita em forma muito simples através das variáveis $k^{\prime}, h^{\prime}, v^{\prime}, u^{\prime}$ do desenvolvimento assimétrico (cf. Eqs. 3.23 e 3.24 ) e suas derivadas.

Por um lado, a partir das Eqs. (4.1) escrevemos

$$
\begin{aligned}
& \bar{k}+i \bar{h}=e_{\mathrm{J}} \exp i \varpi_{\mathrm{J}} \\
& \bar{v}+i \bar{u}=\sin \frac{I_{\mathrm{J}}}{2} \exp i \Omega_{\mathrm{J}}
\end{aligned}
$$

e a partir das Eqs. (4.6) escrevemos

$$
\delta \bar{k}+i \delta \bar{h}=\left(\delta e_{\mathrm{J}}+i e_{\mathrm{J}} \delta \varpi_{\mathrm{J}}\right) \exp i \varpi_{\mathrm{J}}
$$

Por outro lado, a partir da Eq. (4.9), e lembrando que $\psi=\frac{p+q}{q} \lambda_{\mathrm{J}}-\frac{p}{q} \lambda$ (cf. Eqs. 3.3), escrevemos

$$
\delta \psi=\frac{p+q}{q} \delta \lambda_{\mathrm{J}}
$$

Então, temos as relações a seguir

$$
\begin{aligned}
k^{\prime}+i h^{\prime} & =[(\bar{k}+i \bar{h})+(\delta \bar{k}+i \delta \bar{h})] \exp i(\psi+\delta \psi) \\
v^{\prime}+i u^{\prime} & =(\bar{v}+i \bar{u}) \exp i(\psi+\delta \psi)
\end{aligned}
$$


Com a introdução desta variações temporais, a Hamiltoniana do problema passa a depender explicitamente do tempo e vale o que já foi discutido na Seção 3.3. Percebamos, no entanto, que a Eq. (4.17) não só representa uma variação nas variáveis $k^{\prime}, h^{\prime}, v^{\prime}, u^{\prime}$, mas também representa uma variação $\delta \sigma$ e $\delta \sigma_{z}$ nas próprias variáveis do mapeamento (cf. Eqs. 3.3). Estas variações também devem ser introduzidas no desenvolvimento assimétrico e suas derivadas através das variáveis $k, h, v, u$ :

$$
\begin{aligned}
k+i h & =e \exp i(\sigma+\delta \sigma) \\
v+i u & =\sin \frac{I}{2} \exp i\left(\sigma_{z}+\delta \sigma_{z}\right)
\end{aligned}
$$

Portanto, formalmente, as equações do mapeamento (cf. Eqs. 3.27 e 3.28) devem ser escritas na forma

$$
\begin{aligned}
J_{i}^{n+1} & =J_{i}^{n}-\tau \frac{\partial \mathcal{H}^{*}\left(t^{n}, \boldsymbol{\theta}^{n}+\delta \boldsymbol{\theta}^{n}, \mathbf{J}^{n+1}\right)}{\partial \theta_{i}^{n}} \\
\theta_{i}^{n+1} & =\theta_{i}^{n}+\tau \frac{\partial \mathcal{H}^{*}\left(t^{n}, \boldsymbol{\theta}^{n}+\delta \boldsymbol{\theta}^{n}, \mathbf{J}^{n+1}\right)}{\partial J_{i}^{n+1}}, \quad i=1,2,3 . \\
t^{n+1} & =t^{n}+\tau
\end{aligned}
$$

onde $\delta \boldsymbol{\theta}^{n}$ é a variação Eq. (4.17) calculada no instante $t^{n}$. Rigorosamente, a relação certa para a segunda equação é

$$
\left(\theta_{i}^{n+1}+\delta \theta_{i}^{n+1}\right)=\left(\theta_{i}^{n}+\delta \theta_{i}^{n}\right)+\tau \frac{\partial \mathcal{H}^{*}\left(t^{n}, \boldsymbol{\theta}^{n}+\delta \boldsymbol{\theta}^{n}, \mathbf{J}^{n+1}\right)}{\partial J_{i}^{n+1}}
$$

No entanto, como o período da variação $\delta \boldsymbol{\theta}$ é 880 anos, e $\tau \ll 880$ anos, podemos supor que $\delta \theta_{i}^{n+1} \sim \delta \theta_{i}^{n}$ e cancelar em ambos membros estas variações.

Com isto completam-se todos os detalhes relacionados com a construção do mapeamento. Nos capítulos restantes apresentaremos os resultados da nossa pesquisa. 


\section{Resultados}

Este capítulo é dedicado a apresentar os principais resultados da pesquisa desenvolvida. Discutiremos também alguns critérios a serem levados em conta para analisar estes resultados, de forma a se obter a maior quantidade de informação possível sobre o seu comportamento, e visando que essa informação seja suficientemente representativa e completa. Vários conceitos utilizados freqüentemente ao longo deste capítulo são apresentados com maior detalhe no Apêndice A.

\subsection{Análise dos resultados}

É importante esclarecer que, na análise que faremos, devemos ter sempre presente que os resultados que fornece o mapeamento são de caráter qualitativo, e não devemos esperar que eles reproduzam quantitativamente os resultados que são obtidos a partir de integrações precisas. O mapeamento fornece uma primeira aproximação à fenomenologia do problema, e é por isso que o número de testes que devem ser feitos deve ser muito grande para que os resultados possam ser avaliados em função da sua significância estatística.

O mapeamento utilizado fornece a evolução temporal das variáveis $\psi, \sigma, \sigma_{z}$ (Eqs. 3.3) e $P, J, J_{z}$ (Eqs. 3.12). No entanto, para efeitos práticos, resulta mais interessante analisar a evolução temporal de certas grandezas que estão vinculadas mais diretamente com a fenomenologia do problema ressonante. Estas grandezas são: (1) o argumento crítico $\sigma$, cujo comportamento (libração ou circulação) é fornecido diretamente pelo 
mapeamento; (2) o argumento secular $\varpi-\varpi^{\prime}=\psi-\sigma-\varpi^{\prime}$, cujo comportamento está associado à existência de ressonâncias seculares (regiões de corrotação); e (3) o argumento do periélio $\omega=\sigma_{z}-\sigma$, cujo comportamento está vinculado à existência da ressonância de Kozai. Por sua vez, é claro que resulta mais simples interpretar o comportamento dos elementos orbitais $a, e, I$ em função do tempo, do que analisar as variáveis $P, J, J_{z}$. Uma última grandeza cuja evolução temporal é interessante analisar é o valor da própria hamiltoniana média $\mathcal{H}^{*}$.

Como estamos estudando um sistema com muitos graus de liberdade, não é possível fazer-se um análise da sua evolução temporal em todo o espaço de fase. Podemos então utilizar superfícies de seção que permitam visualizar o comportamento do sistema em gráficos bidimensionais, onde a interpretação dos resultados pode ser feita em forma simples e não ambígua. A escolha desta superfície de seção pode ser arbitrária. Porém, aquelas superfícies vinculadas às características dinâmicas do problema, apresentam maior interesse que as outras. No caso de uma ressonância de primeira ordem, uma destas superfícies de seção pode ser definida pelas condições simultâneas

$$
\begin{array}{lll}
\sigma=0 & , & \dot{\sigma}>0 \\
\varpi-\varpi^{\prime}=0, & \dot{\varpi}-\dot{\varpi}^{\prime}>0 \\
\omega=\pi / 2 & , \quad & \dot{\omega}>0 .
\end{array}
$$

A primeira condição está vinculada aos pontos de equilíbrio estável da ressonância; a segunda está vinculada aos pontos de corrotação na ressonância; e a terceira, aos pontos de equilíbrio estável da ressonância de Kozai. É importante mencionar que esta superfície de seção não constitui um mapa de Poincaré do sistema, já que na sua definição não fazemos uso de nenhuma integral primeira.

$\mathrm{Na}$ construção desta superfície de seção devemos levar em conta três coisas. A primeira é que, para que as condições Eqs. (5.1) se repitam periodicamente, em forma exata e simultânea, os períodos de libração ou circulação de $\sigma, \varpi-\varpi^{\prime}, \omega$ devem ser comensuráveis ${ }^{1}$. Mesmo assim, poderia acontecer que o intervalo de tempo para que as condições se repitam seja da ordem ou maior que o intervalo total de integração, em cujo caso obteríamos uma superfície de seção com muito poucos pontos. Em segundo lugar, devemos levar em conta que nossos resultados são discretos e portanto, as condições Eqs. (5.1) cumprir-se-ão só em forma aproximada. Finalmente, devemos levar em conta

\footnotetext{
${ }^{1}$ Caso contrário, as três condições não se repetiriam nunca en forma simultânea.
} 
que no caso em que $\varpi-\varpi^{\prime}$ e $\omega$ circulam, o sinal das freqüências instantâneas $\dot{\varpi}-\dot{\varpi}^{\prime} \mathrm{e}$ $\dot{\omega}$ dependerá do tipo de circulação: direta ou retrógrada.

$\mathrm{Na}$ nossa aplicação, fizemos várias provas e achamos que uma superfície de seção adequada para representar os resultados do mapeamento é definida por

$$
|\sigma|<1^{\circ}, \quad\left|\omega-90^{\circ}\right|<1^{\circ} .
$$

Os sinais das freqüências são fixados em função dos sinais das freqüências iniciais $\dot{\sigma}_{\text {ini }}, \dot{\varpi}_{\text {ini }}$ e $\dot{\omega}_{\text {ini }}$, e estas condições são monitoradas a cada passo do mapeamento. Na maior parte dos casos estudados, esta superfície de seção fornece um número de resultados suficiente para obter uma boa representação do comportamento do sistema. Quase todas a figuras mostradas neste capítulo são representadas nesta superfície.

\subsection{A ressonância $2 / 1$}

Na Fig. 5.1 os pontos cinzas representam a grade das 165 condições iniciais fictícias no espaço $a, e$ que foram testadas com o mapeamento na ressonância 2/1. Para todas estas condições a inclinação inicial foi $I=5^{\circ}$ (lembremos que a inclinação não pode ser nula pela singularidade das equações do mapeamento). Os valores dos ângulos iniciais foram escolhidos sobre a superfície de seção $\sigma=0^{\circ}, \varpi-\varpi^{\prime}=0^{\circ}$ e $\omega=90^{\circ}$. A figura apresenta também a posição do ramo pericêntrico a das separatrizes da ressonância, junto com a posição de algumas ressonâncias seculares $\left(\nu_{5}, \nu_{16}\right)$, de algumas ressonâncias secundárias $\left(f_{\sigma} / f_{\varpi}\right)$, e da borda inferior $(L)$ do complexo das ressonâncias pericêntricas (ver Apêndice A, Seção A.2). Finalmente, a Fig. 5.1 mostra também a posição, sobre a mesma superfície de seção, dos asteróides com boas observações na ressonância, isto é, levando em conta só os objetos numerados e multi-oposicionais da base de dados de Bowell et al. (1994) (atualizada a 1997, cf. Tabela 5.1). Estes objetos são representados pelos pontos mais escuros, e são caracterizados segundo o tipo de comportamento que possuem (muito regular ou caótico, respectivamente).

Esta grade de condições iniciais foi integrada por um intervalo de tempo de $10^{8}$ anos. A integração de uma dada condição era interrompida quando o objeto escapava. Para avaliar a ocorrência de um escape, foram realizados controles sobre os valores dos elementos orbitais a cada passo do mapeamento. Estes controles consistiram em:

1. avaliar se a excentricidade do objeto atinge $e>0.65$. É claro que se objeto atinge 


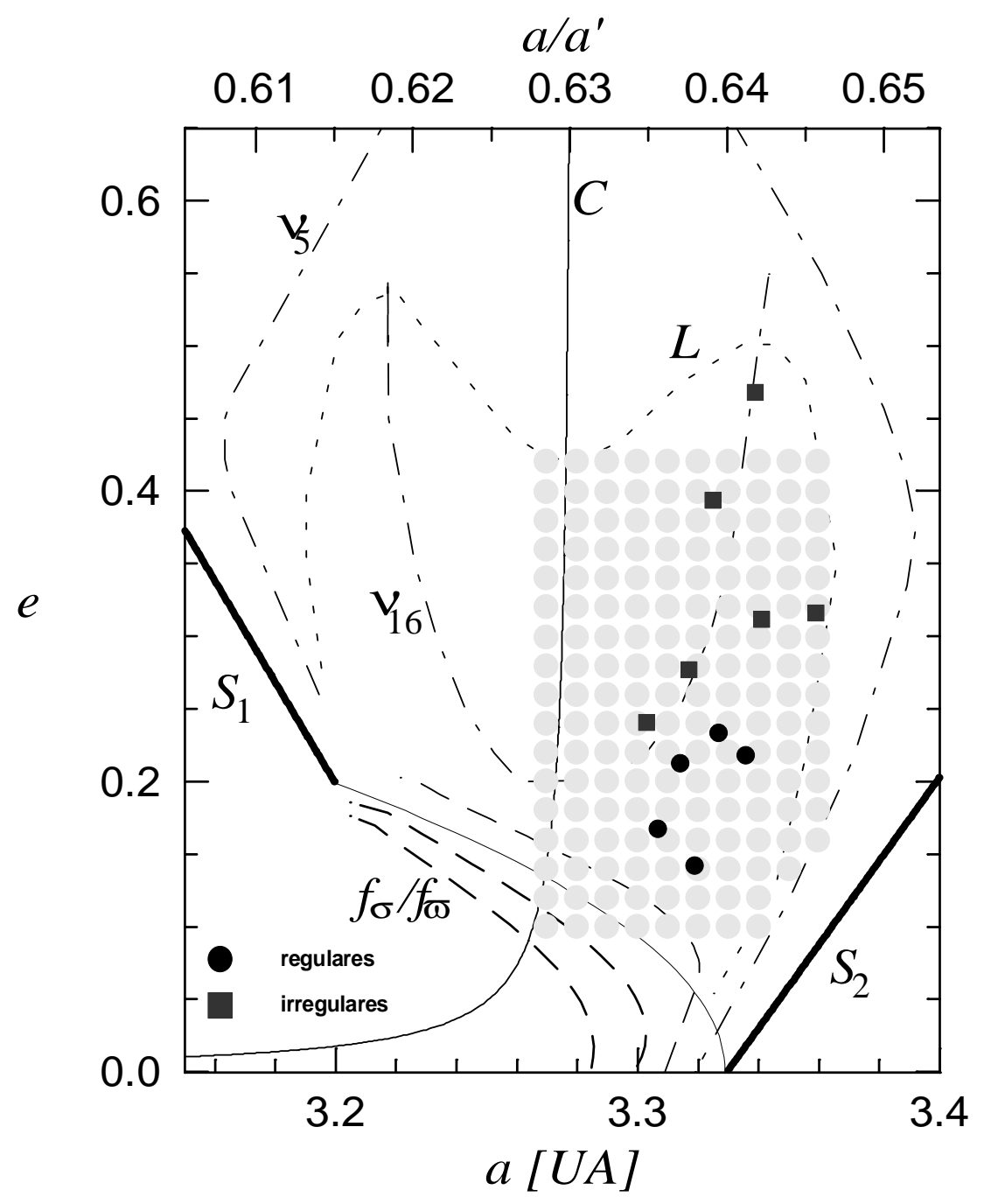

Figura 5.1: Ressonância 2/1. Condições iniciais testadas com o mapeamento por $10^{8}$ anos (pontos cinzas). Em todos os casos $I=5^{\circ}, \sigma=0^{\circ}, \varpi-\varpi^{\prime}=0^{\circ}$ e $\omega=90^{\circ}$. Os pontos mais escuros representam as posições dos asteróides bem observados (numerados e multi-oposicionais) na ressonância: círculos representam os que são muito estáveis e quadros representam os que são caóticos (a inclinação destes objetos é variada e pode ser conferida na Tabela 5.1). As linhas de ressonâncias seculares e secundárias apresentadas nesta figura foram calculadas para a superfície $I=0, \sigma=0, \varpi-\varpi^{\prime}=0$ e $\Omega-\Omega^{\prime}=0$ (Michtchenko e Ferraz-Mello, 1997). 


\begin{tabular}{rlccc}
\hline \hline & Nome & $a[\mathrm{UA}]$ & $e$ & $I$ \\
\hline \multirow{2}{*}{ Irregulares } & & & & \\
1362 & Griqua & 3.359 & 0.316 & $24^{\circ}$ \\
1921 & Pala & 3.341 & 0.312 & $19^{\circ}$ \\
1922 & Zulu & 3.325 & 0.394 & $39^{\circ}$ \\
3688 & Navajo & 3.339 & 0.468 & $3^{\circ}$ \\
4177 & 1987 SS1 & 3.317 & 0.277 & $18^{\circ}$ \\
& 1981 EX11 & 3.303 & 0.241 & $18^{\circ}$ \\
& & & & \\
Regulares & & & & \\
3789 & Zhongguo & 3.307 & 0.167 & $2^{\circ}$ \\
& 1975 SX & 3.319 & 0.142 & $3^{\circ}$ \\
& 1990 TH7 & 3.336 & 0.218 & $1^{\circ}$ \\
& 1993 SK3 & 3.327 & 0.233 & $2^{\circ}$ \\
& 1994 UD1 & 3.314 & 0.213 & $3^{\circ}$ \\
\hline \hline
\end{tabular}

Tabela 5.1: Asteróides bem observados na ressonância 2/1 (numerados e multioposicionais), apresentados na Fig. 5.1. Quase todos os objetos com inclinações altas se encontram sobre a ressonância secular $\nu_{16}$.

essa excentricidade fica bem no meio da região altamente caótica do complexo de ressonâncias pericêntricas, e será ejetado da ressonância em pouco tempo.

2. avaliar se $|\sigma|>90^{\circ}$ e $e>0.5$ simultaneamente. Nesse caso o objeto encontra-se muito perto da curva de colisão do problema plano. Além de existir a possibilidade de um encontro próximo ("close approach") com Júpiter, sabemos que sob essas condições, o desenvolvimento assimétrico fornece uma representação ruim da função perturbadora.

3. avaliar se a inclinação $I>40^{\circ}$. Novamente o nosso modelo não é adequado para ser utilizado com inclinações tão altas. O leitor pode argumentar que esta condição não representa na verdade um escape. Porém, podemos contestar dizendo que se o objeto atinge uma inclinação tão alta em intervalos de tempo curtos (como foi observado em todos estes tipos de "escapes"), isto deve-se à existência de uma difusão importante na terceira dimensão, que sem dúvida está vinculada a um fenômeno estocástico.

Os primeiros resultados foram obtidos considerando-se só a existência de variações seculares na órbita de Júpiter, isto é, não levando em conta os termos da comensura- 
bilidade 5/2 Júpiter-Saturno. Estes resultados são apresentados na Fig. 5.2a,b. A Fig. 5.2a mostra as condições iniciais que escaparam durante a integração, utilizando pontos pretos, cinzas e brancos para distinguir entre os três tipos de escape mencionados acima, respectivamente. Os resultados mostram que a maior parte das condições não escapam, exceto aquelas que se acham: (1) sobre a teia de ressonâncias secundárias; (2) perto da borda inferior do complexo pericêntrico (curva $L$ ); e (3) na região mais próxima da separatriz. Isto está em total acordo com o que é conhecido a partir de outros estudos analíticos e numéricos (cf. Apêndice A). A Fig. 5.2b mostra o tempo de escape dos objetos como função do semieixo inicial. Os objetos estão diferenciados em função da sua excentricidade inicial: triângulos para objetos com $e_{0}<0.2$, cruzes para $0.2 \leq e_{0}<0.3$, e rombos para $e_{0} \geq 0.3$. Percebamos que na região do ramo pericêntrico, os tempos de escape estão bem diferenciados dependendo da excentricidade inicial: os objetos sobre as ressonâncias secundárias demoram muito mais em escapar que os que se acham sobre a borda $L$, cujo tempo de sobrevivência é apenas 10000 anos. Novamente isto está em bom acordo com o que é conhecido através de outros estudos numéricos.

O passo seguinte consistiu em integrar a mesma grade de condições (por $10^{8}$ anos), mas agora levando em conta as variações seculares de Júpiter junto com as variações periódicas da comensurabilidade $5 / 2$. Lembremos que o valor real da freqüência $5 n_{\mathrm{S}}-$ $2 n_{\mathrm{J}}$ destas variações periódicas corresponde a um período de $\sim 880$ anos. A Fig. 5.3a,b apresenta os resultados obtidos. Comparando com a Fig. 5.2a, é notória a diferença no número de objetos que escapam, particularmente na região de excentricidades intermédias em torno do ramo pericêntrico. Este tipo de comportamento já fora observado com o mapa plano de Ferraz-Mello (1997). Apesar de que integrações numéricas mais realísticas não refletem a mesma sensibilidade às perturbações da comensurabilidade $5 / 2$, elas indicam que a difusão nessa região do espaço de fase existe mas é mais lenta. Insistimos em que os resultados do mapeamento possuem um caráter só qualitativo, e que o que nos observamos é uma indicação de que a comensurabilidade $5 / 2$ cumpre um papel importante na evolução dinâmica do problema. É precisamente este tipo de informação a que orientou-nos no rumo que demos à pesquisa no que resta desta tese.

O que decidimos fazer foi utilizar nosso mapeamento como "toy-model" para estudar 


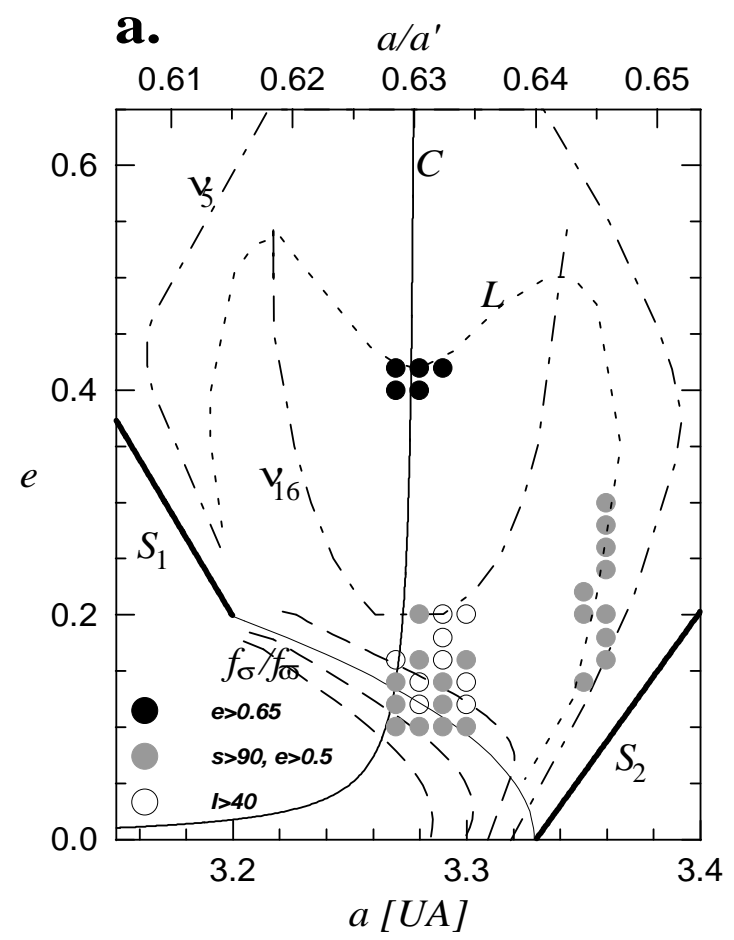

b.

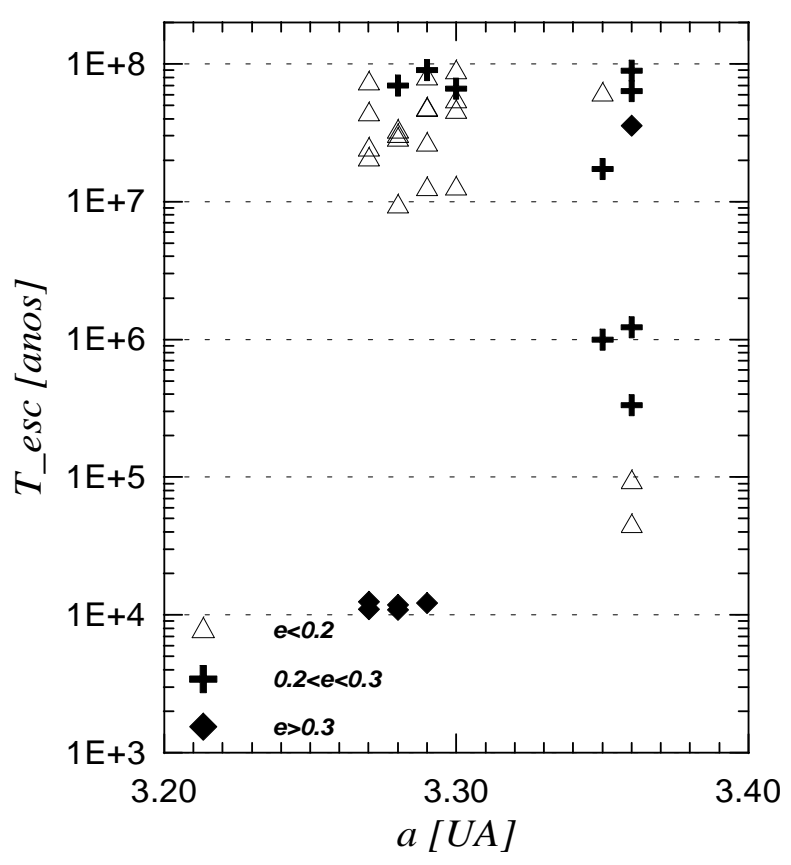

Figura 5.2: Ressonância 2/1. Condições iniciais que escaparam durante a integração com o mapeamento levando em conta só as perturbações seculares na órbita de Júpiter. (a) Distribuição no plano $a, e$ : os círculos pretos representam escapes com $e>0.65$; os círculos cinzas são escapes com $|\sigma|>90^{\circ}$ e $e>0.5$; os círculos brancos são escapes com $I>40^{\circ}$. (b) Tempo de escape em função do semieixo inicial: os triângulos são objetos inicialmente com $e<0.2$; as cruzes são objetos inicialmente com $0.2 \leq e<0.3$; os rombos são objetos inicialmente com $e \geq 0.3$. 


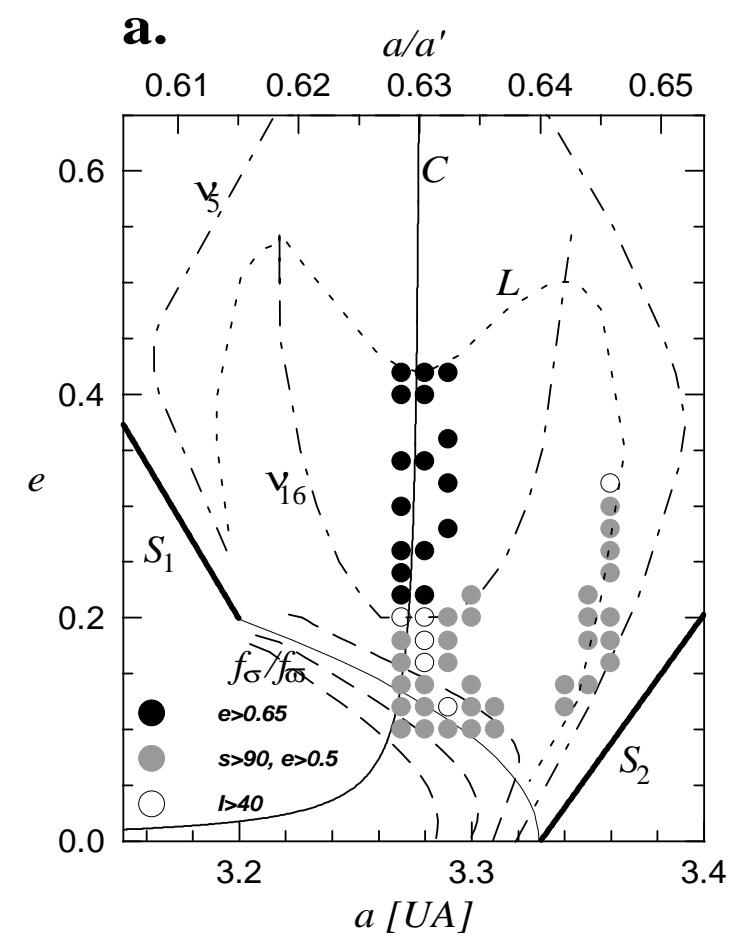

b.

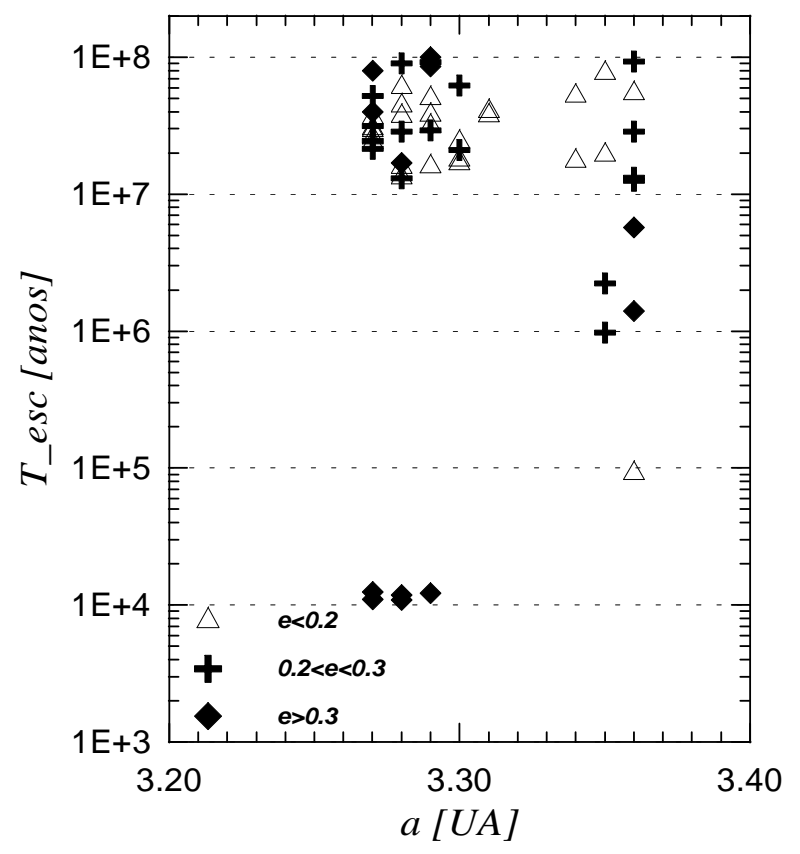

Figura 5.3: Igual que a Fig. 5.2, mas agora adicionando as perturbações de curto período na órbita de Júpiter devidas à comensurabilidade $5 / 2$ entre este planeta e Saturno. A freqüência destas perturbações é $5 n_{\mathrm{S}}-2 n_{\mathrm{J}} \simeq 1470^{\prime \prime} /$ ano e seu período é aproximadamente 880 anos. 
com mais detalhe a mudança da dinâmica da ressonância $2 / 1$ frente às mudanças na freqüência $5 n_{\mathrm{S}}-2 n_{\mathrm{J}}$. A integração das 165 condições iniciais foi repetida seis vezes mais, mudando-se cada vez o valor da citada freqüência. Os resultados são apresentados nas Figs. 5.4 - 5.9.

A Fig. 5.4a,b corresponde ao caso em que a freqüência utilizada foi 7 vezes maior que a freqüência real, o que representa um período de $\sim 126$ anos. Este caso é equivalente a afastar Júpiter e Saturno da condição de ressonância $5 / 2$ exata. O que podemos observar nos resultados é que eles não são muito diferentes do caso em que as perturbações da ressonância 5/2 não são levadas em conta (Fig. 5.2).

Na Fig. 5.5a,b o valor de freqüência utilizado foi 5 vezes maior que o valor real (período 176 anos). Agora estamos começando a aproximar Júpiter e Saturno da condição de ressonância exata. Observamos que não existem diferenças com as Figs. 5.2 e 5.4, exceto por algumas condições iniciais perto e sobre a ressonância secular $\nu_{16}$ que escapam. Mais adiante, analisaremos com mais detalhe o comportamento destas condições.

Na Fig. 5.6a,b o valor da freqüência foi 3 vezes maior que o real $(\sim 293$ anos de período). A diferença mais notória com os casos anteriores é o grande número de objetos que escapam na região de $0.3<e<0.4$ compreendida entre a ressonância secular $\nu_{16} \mathrm{e}$ a borda $L$. O escape destes objetos ocorre em intervalos de tempo relativamente curtos ( 1000000 de anos) e em geral apresentam uma difusão importante em inclinação. Uma característica interessante dos objetos nessa região é que o período de libração do argumento crítico $\sigma$ é aproximadamente 300 anos (Nesvorný e Ferraz-Mello, 1997b). Este valor é muito parecido com o período da freqüência $5 n_{\mathrm{S}}-2 n_{\mathrm{J}}$ que foi utilizada. A questão é: será que o batimento entre as duas freqüências contribui para a aceleração dos processos difusivos? Infelizmente, o mapeamento não permite responder a esta pergunta, mas novamente indica o caminho a seguir em futuras pesquisas com modelos mais precisos.

$\mathrm{O}$ caso apresentado na Fig. 5.7a,b é o mais notório de todos. A frequëncia $5 n_{\mathrm{S}}-2 n_{\mathrm{J}}$ é neste caso duas vezes maior que seu valor real, o que representa um período de $\sim 440$ anos. Esta configuração foi analisada por Michtchenko e Ferraz-Mello (1997) através de integrações numéricas precisas. Comparando esta figura com a Fig. 5.3 vemos que a região central em torno do ramo pericêntrico é fortemente esvaziada em intervalos 


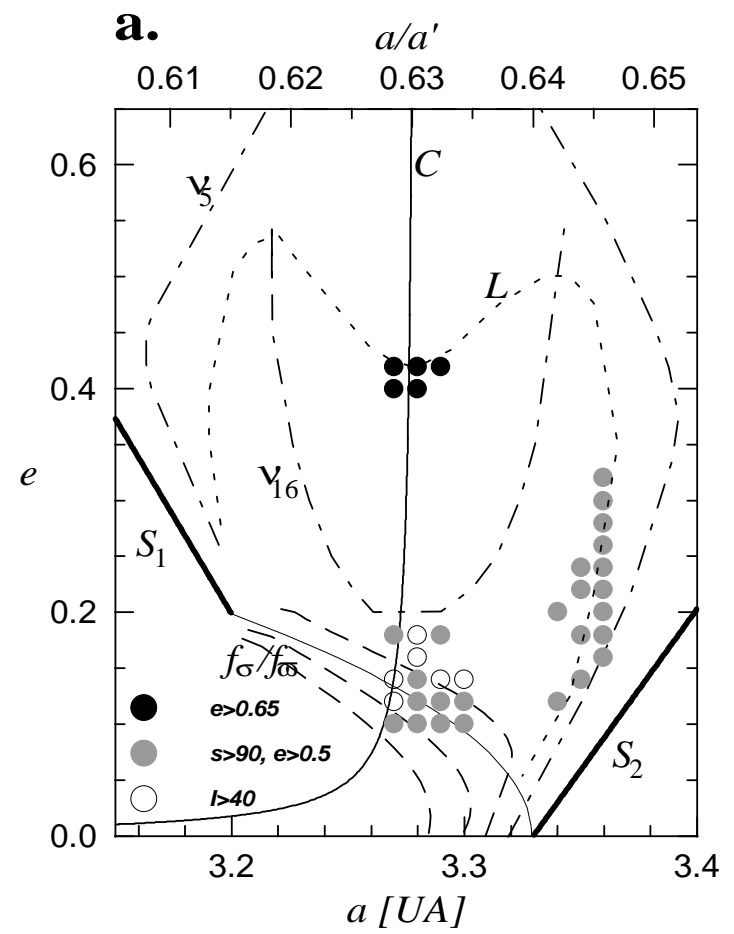

b.

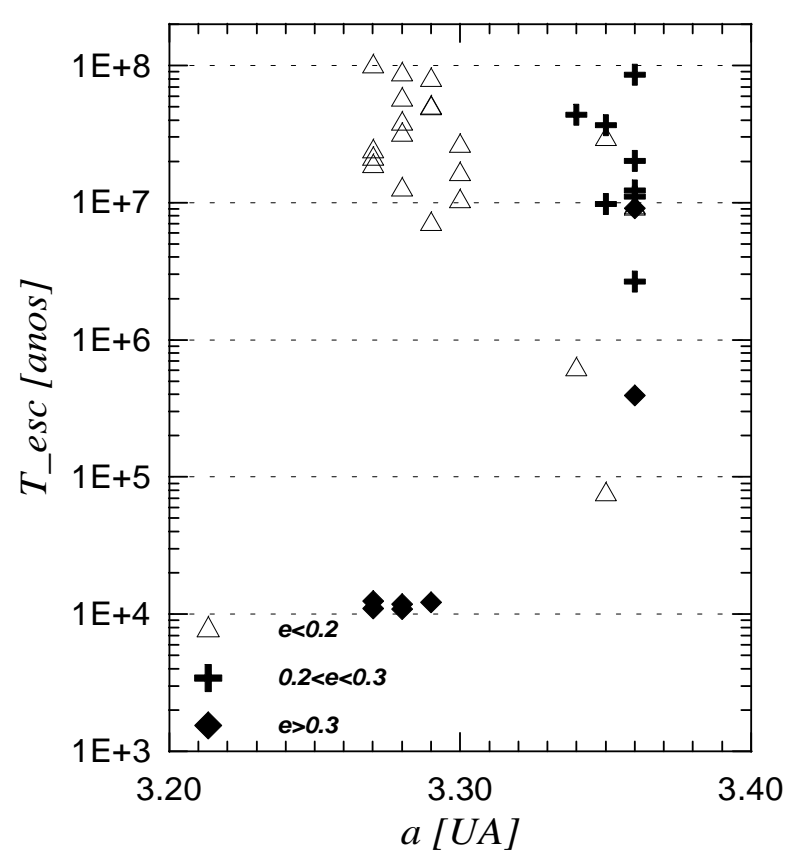

Figura 5.4: Igual que a Fig. 5.3, considerando um valor de $5 n_{\mathrm{S}}-2 n_{\mathrm{J}}=10290^{\prime \prime} /$ ano (aproximadamente 126 anos de período). 


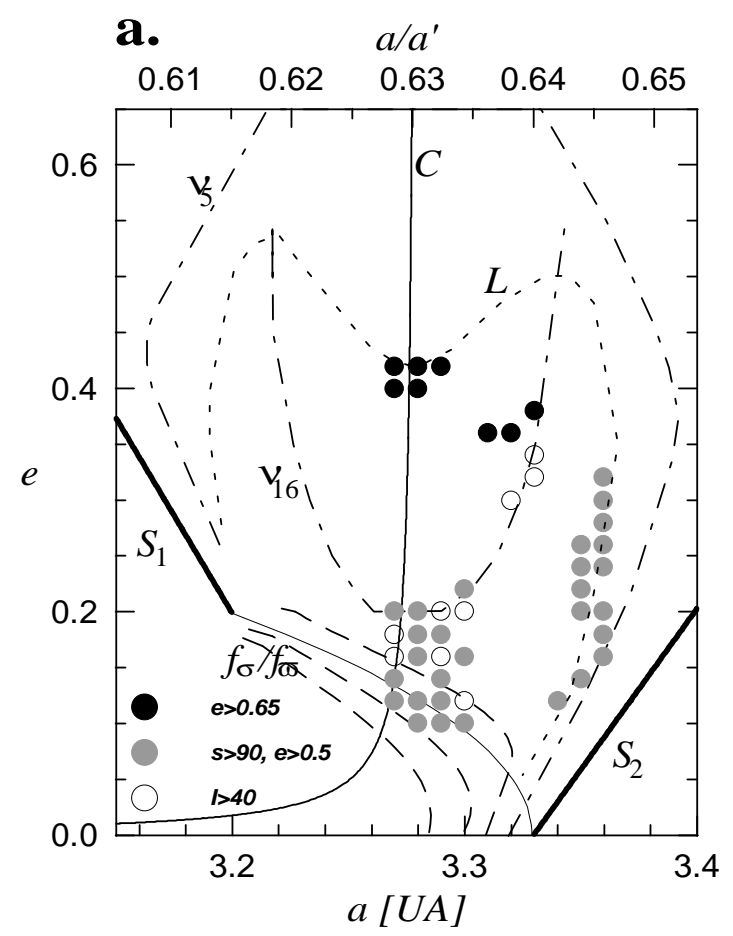

b.

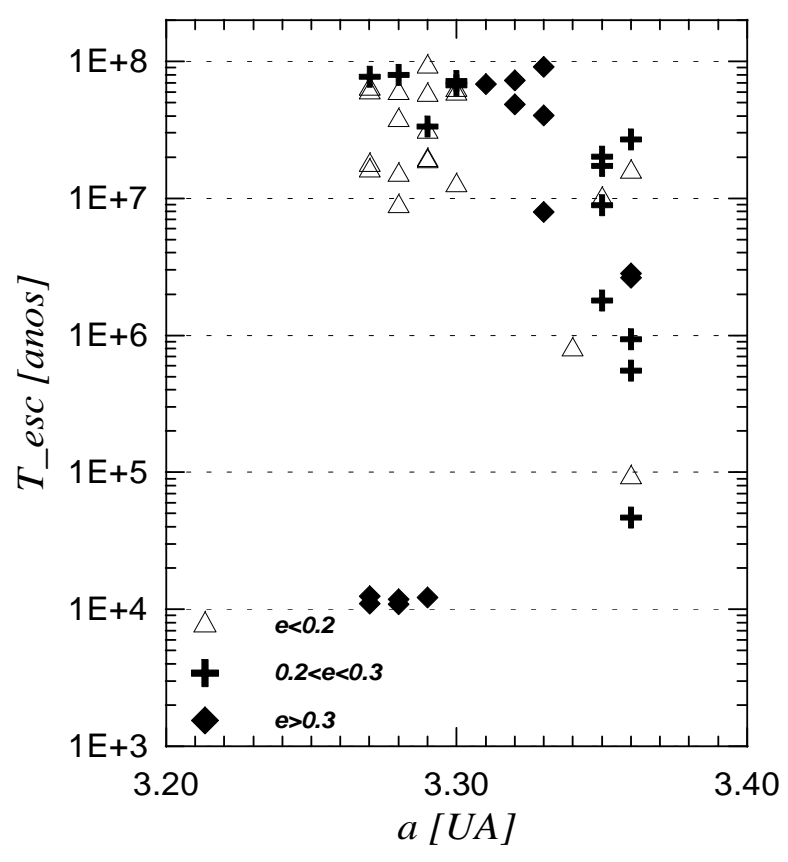

Figura 5.5: Igual que a Fig. 5.3, considerando um valor de $5 n_{\mathrm{S}}-2 n_{\mathrm{J}}=7350^{\prime \prime} /$ ano (aproximadamente 176 anos de período). 


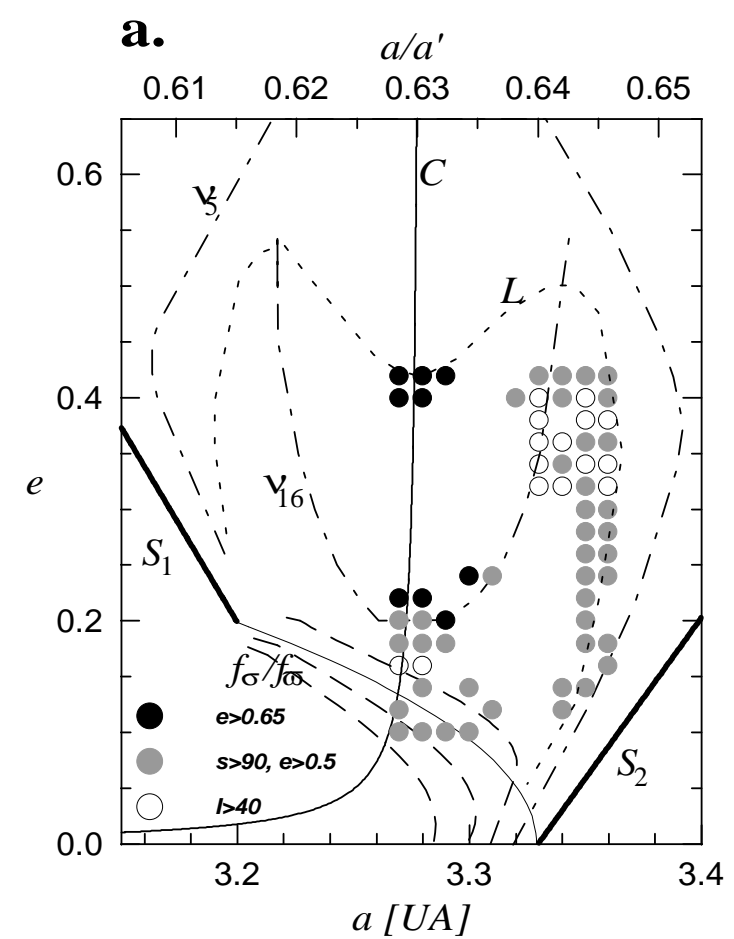

b.

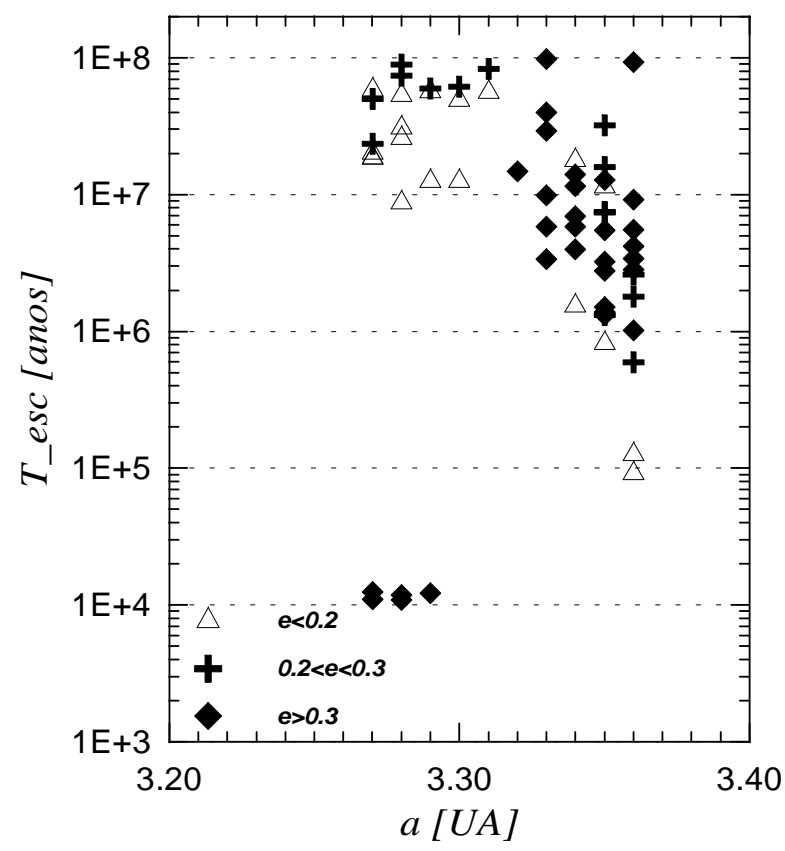

Figura 5.6: Igual que a Fig. 5.3, considerando um valor de $5 n_{\mathrm{S}}-2 n_{\mathrm{J}}=4410^{\prime \prime} /$ ano (aproximadamente 293 anos de período). 
de tempo de $\sim 10^{7}$ anos. Precisamente nessa região, o período de libração de $\sigma$ é aproximadamente 440 anos, o que nos leva novamente a hipótese do batimento entre freqüências como responsável pela aceleração da difusão. O comportamento é similar quando utilizamos um valor de $5 n_{\mathrm{S}}-2 n_{\mathrm{J}}$ igual a 1.5 vezes seu valor real ( $\sim 587$ anos de período). Este caso é apresentado na Fig. 5.8a,b. Comparando-o com o caso anterior, o esvaziamento da região central não é tão notório, mas continua sendo maior que no caso da Fig. 5.3.

A última série de testes foi realizada utilizando um valor de $5 n_{\mathrm{S}}-2 n_{\mathrm{J}}$ igual a 0.75 vezes o valor real, que equivale a uns 1173 anos de período. Este valor de freqüência corresponde a uma circulação que está muito próxima da separatriz da ressonância $5 / 2$ Júpiter-Saturno, ou seja que nos achamos no caso em que os dois planetas estão muito próximos da condição ressonante. Os resultados são apresentados na Fig. 5.9a,b. O interessante é que o número de objetos que escapa é menor que no caso em que consideramos a freqüência real (Fig. 5.3), particularmente na região central.

Algumas características globais do comportamento observado em todos estes testes com diferentes valores da freqüência da comensurabilidade $5 / 2$, são as seguintes:

- existem três regiões que se apresentam como sendo muito robustas, no sentido de que elas não são praticamente afetadas pelas variações na freqüência $5 n_{\mathrm{S}}-2 n_{\mathrm{J}}$. Duas delas, a região das ressonâncias secundárias e a região próxima da separatriz, apresentam um comportamento irregular sempre. A terceira é a região de $e \lesssim 0.3$ limitada pelas duas regiões anteriores, que se apresenta como sendo extremamente estável em todos os casos. Esta última corresponde a amplitudes de libração moderadas $\left(\sigma \sim 80^{\circ}\right)$. Observando a Fig. 5.1, percebemos que precisamente nessa região de estabilidade é onde se encontra o grupo de asteróides com comportamento regular observados na ressonância (!)

- existem duas regiões que são muito sensíveis às variações da freqüência $5 n_{\mathrm{S}}-2 n_{\mathrm{J}}$ : a região em torno do ramo pericêntrico para $e \gtrsim 0.2$ (ver Fig. 5.7a) e a região entre as curvas $L$ e $\nu_{16}$ para $e \gtrsim 0.3$ (ver Fig. 5.6a)

- em geral, o tempo de escape é maior para os objetos que se acham inicialmente perto do ramo pericêntrico do que para aqueles que se acham longe e, portanto, possuem amplitudes de libração grandes. 


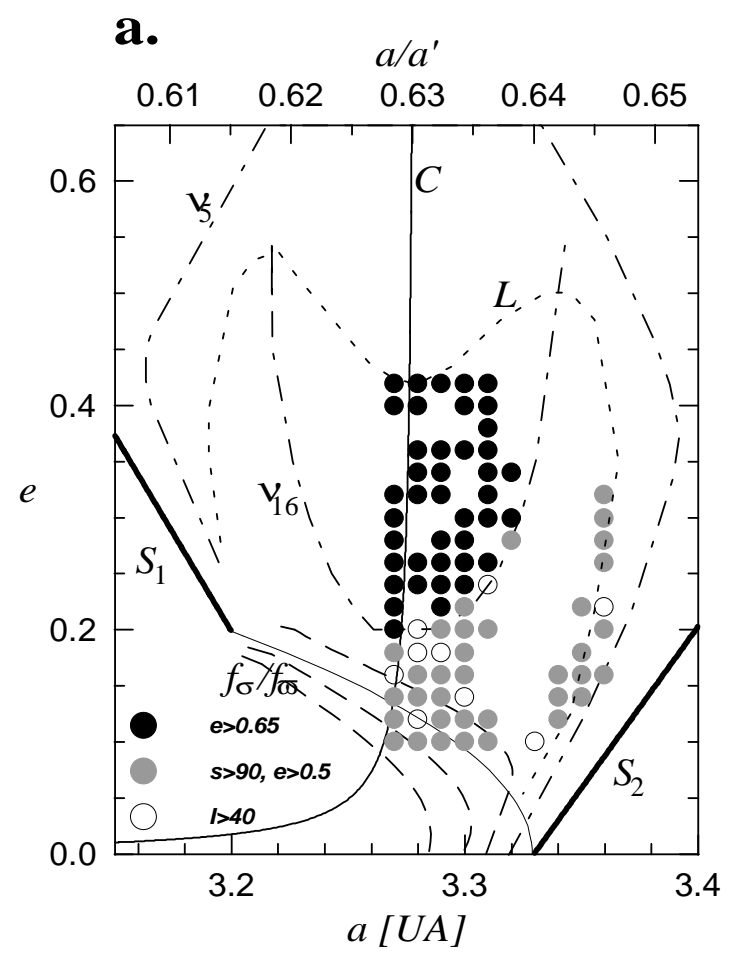

b.

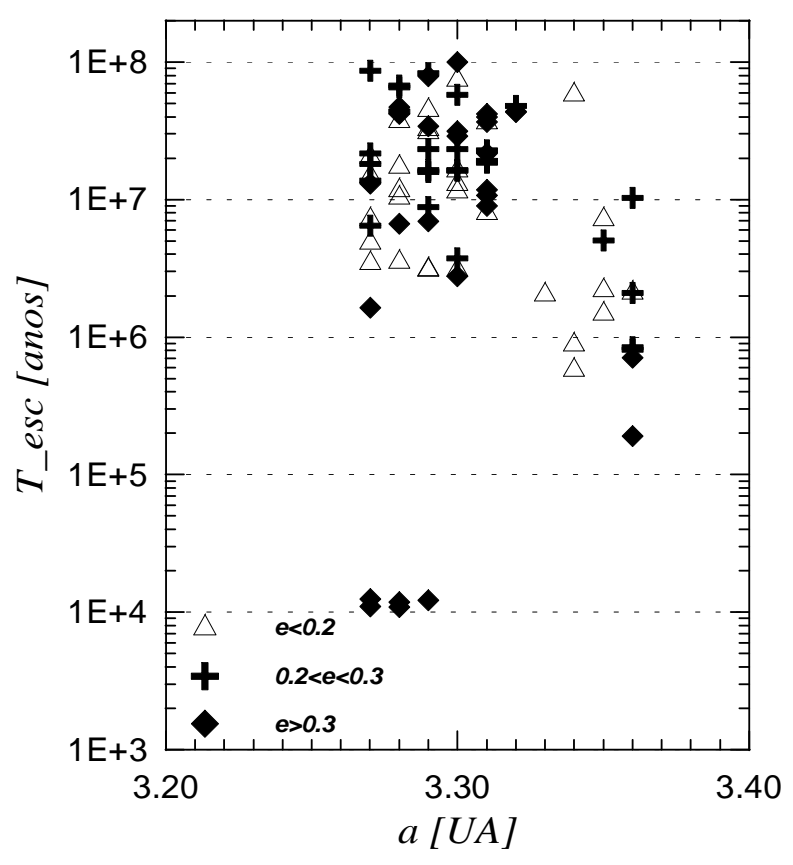

Figura 5.7: Igual que a Fig. 5.3, considerando um valor de $5 n_{\mathrm{S}}-2 n_{\mathrm{J}}=2940^{\prime \prime} /$ ano (aproximadamente 440 anos de período). 


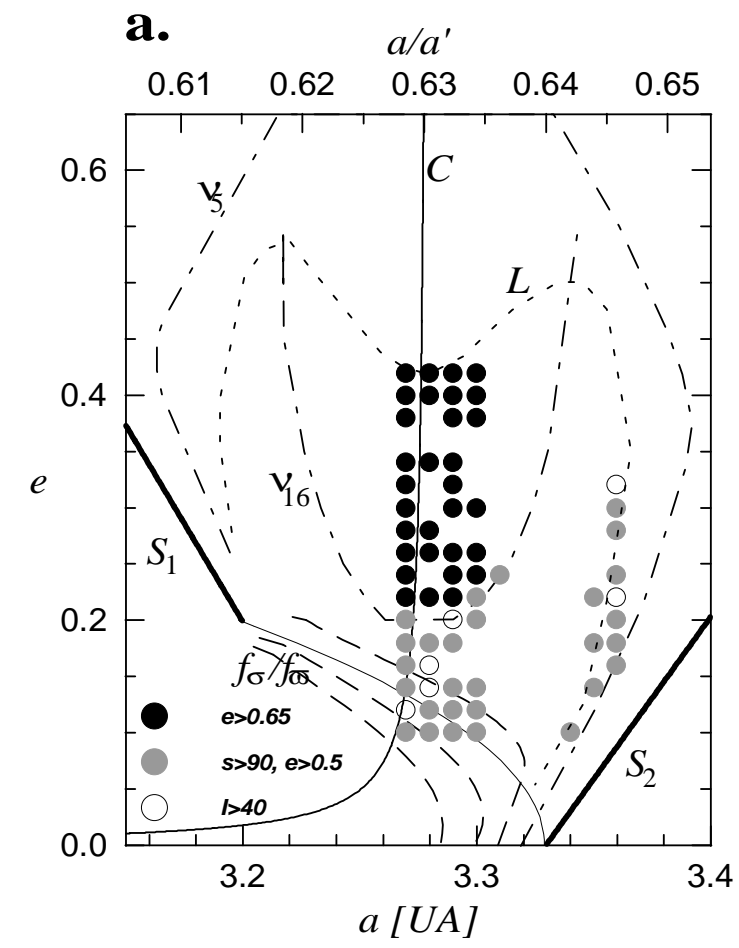

b.

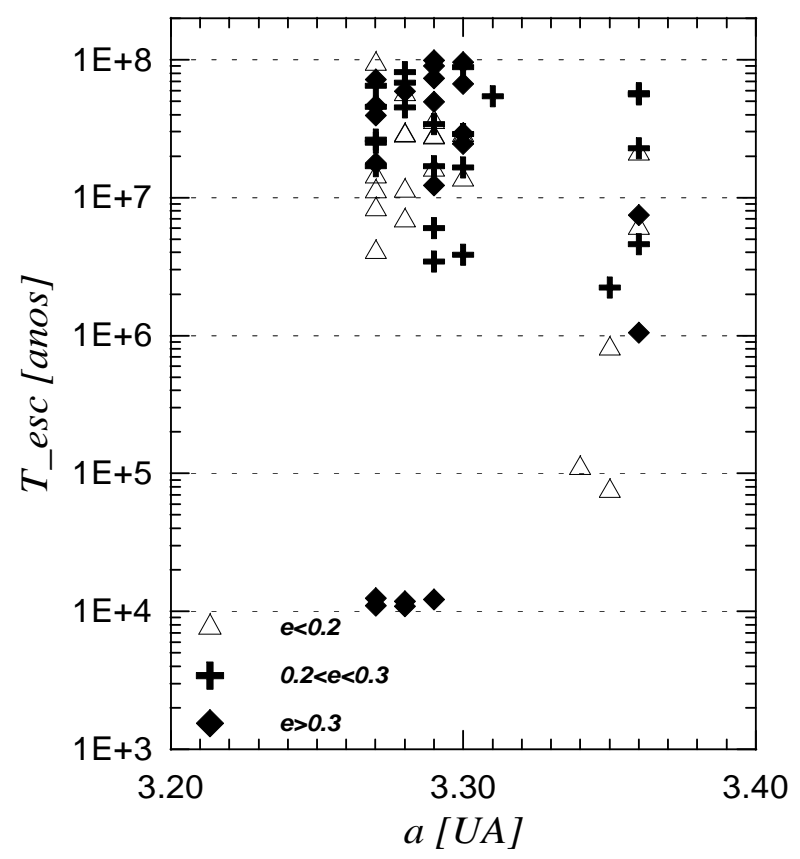

Figura 5.8: Igual que a Fig. 5.3, considerando um valor de $5 n_{\mathrm{S}}-2 n_{\mathrm{J}}=2205^{\prime \prime} /$ ano (aproximadamente 587 anos de período). 

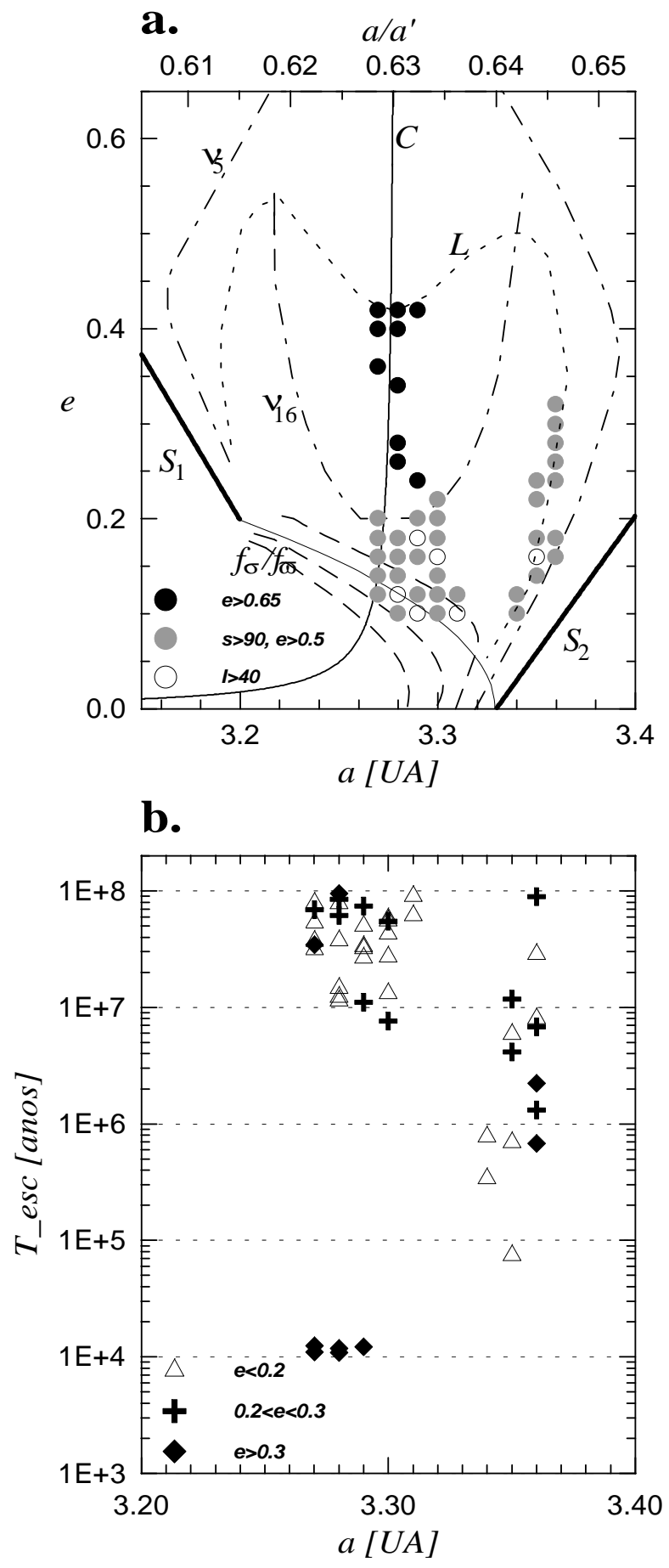

Figura 5.9: Igual que a Fig. 5.3, considerando um valor de $5 n_{\mathrm{S}}-2 n_{\mathrm{J}}=1102^{\prime \prime} /$ ano (aproximadamente 1173 anos de período). 
Até aqui, os resultados obtidos podem ser resumidos no histograma da Fig. 5.10 que apresenta o número de objetos que escapam (diferenciando os três tipos possíveis de escape) em função do período da perturbação da comensurabilidade $5 / 2$. É particularmente notório o máximo que se apresenta quando este período coincide com o da libração de $\sigma$. Devido ao grande número de testes feitos, isto constitui um resultado estatisticamente significativo, e serve para sustentar a hipótese do batimento de freqüências como um mecanismo de esvaziamento importante da ressonância 2/1.

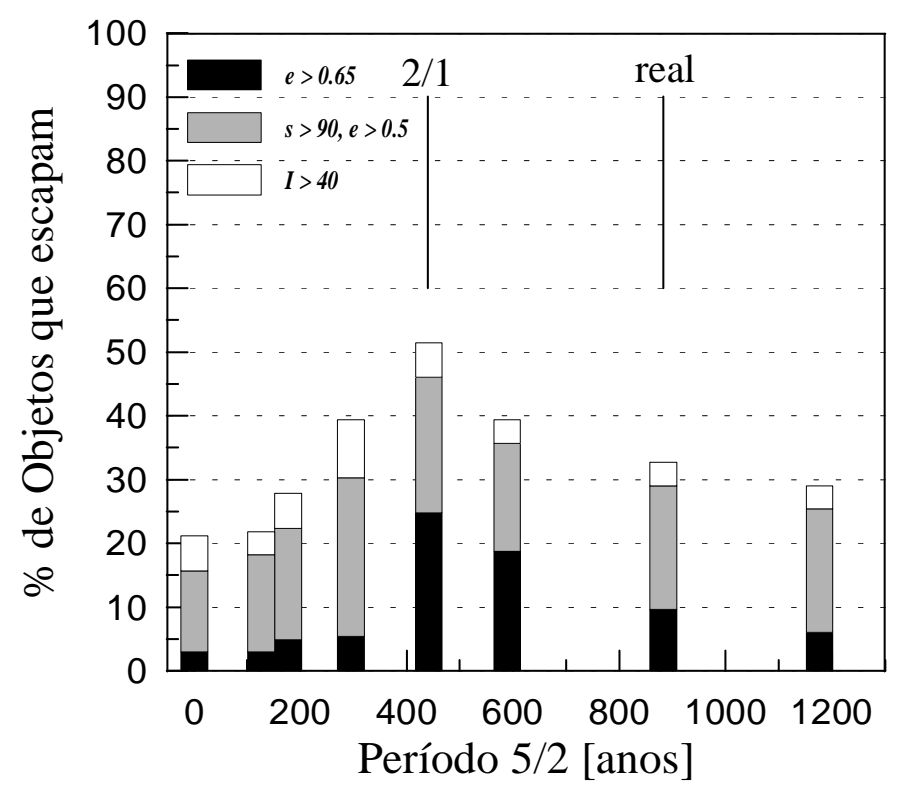

Figura 5.10: Ressonância 2/1. Porcentagem de objetos que escapam (sobre o total das 165 condições testadas) em $T<10^{8}$ anos, como função do valor do período $2 \pi /\left(5 n_{\mathrm{S}}-2 n_{\mathrm{J}}\right)$ das perturbações devidas à comensurabilidade $5 / 2$ entre Júpiter e Saturno. Os diferentes tons de cinza permitem distinguir entre os diferentes tipos de escape. Aparecem indicados os valores do período real da comensurabilidade $5 / 2$ e do período de libração de $\sigma$ na ressonância $2 / 1$.

A questão remanente refere-se às condições dinâmicas sob as quais pode ocorrer uma variação suficientemente importante da freqüência $5 n_{\mathrm{S}}-2 n_{\mathrm{J}}$. Isto fica além da pesquisa desenvolvida nesta tese. Porem, podemos fazer algumas estimações. A Fig. 5.11 apresenta a variação porcentual no semieixo de Saturno (considerando o de Júpiter fixo) como função do período da comensurabilidade $5 / 2$, calculado diretamente usandose a lei de Kepler. Observamos que para varrer um intervalo significativo de freqüências (entre as linhas tracejadas) é preciso uma variação em $a_{\mathrm{S}}$ bem menor que $1 \%$, o que é certamente muito pouco. 


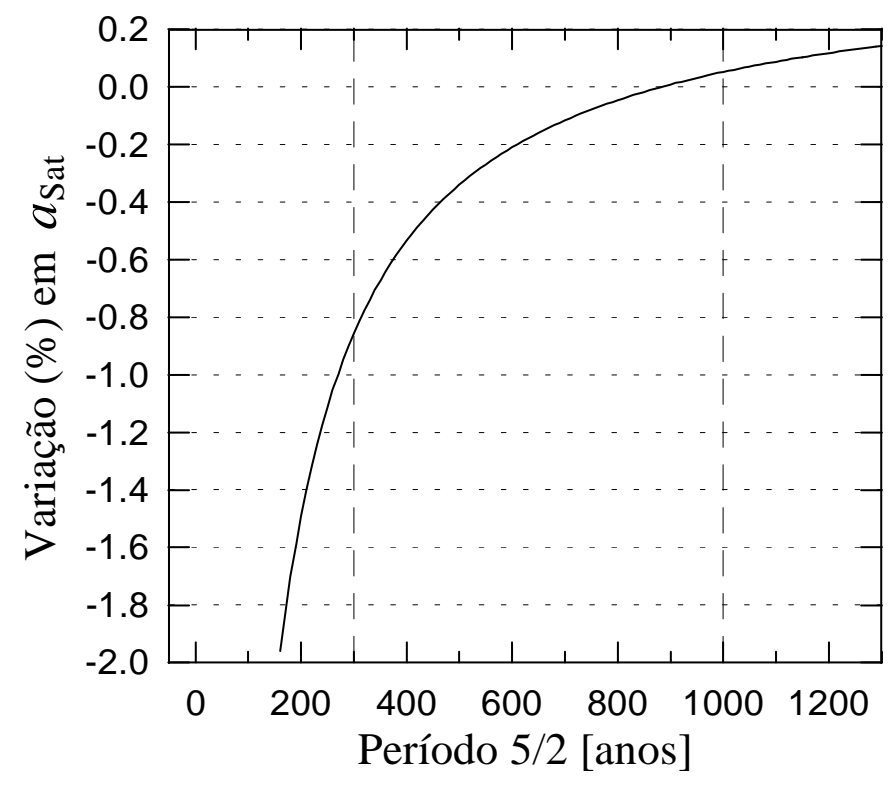

Figura 5.11: Variação porcentual no semieixo de Saturno em função do período da freqüência $5 n_{\mathrm{S}}-2 n_{\mathrm{J}}$. A estimação é feita a partir da lei de Kepler considerando o semieixo de Júpiter fixo.

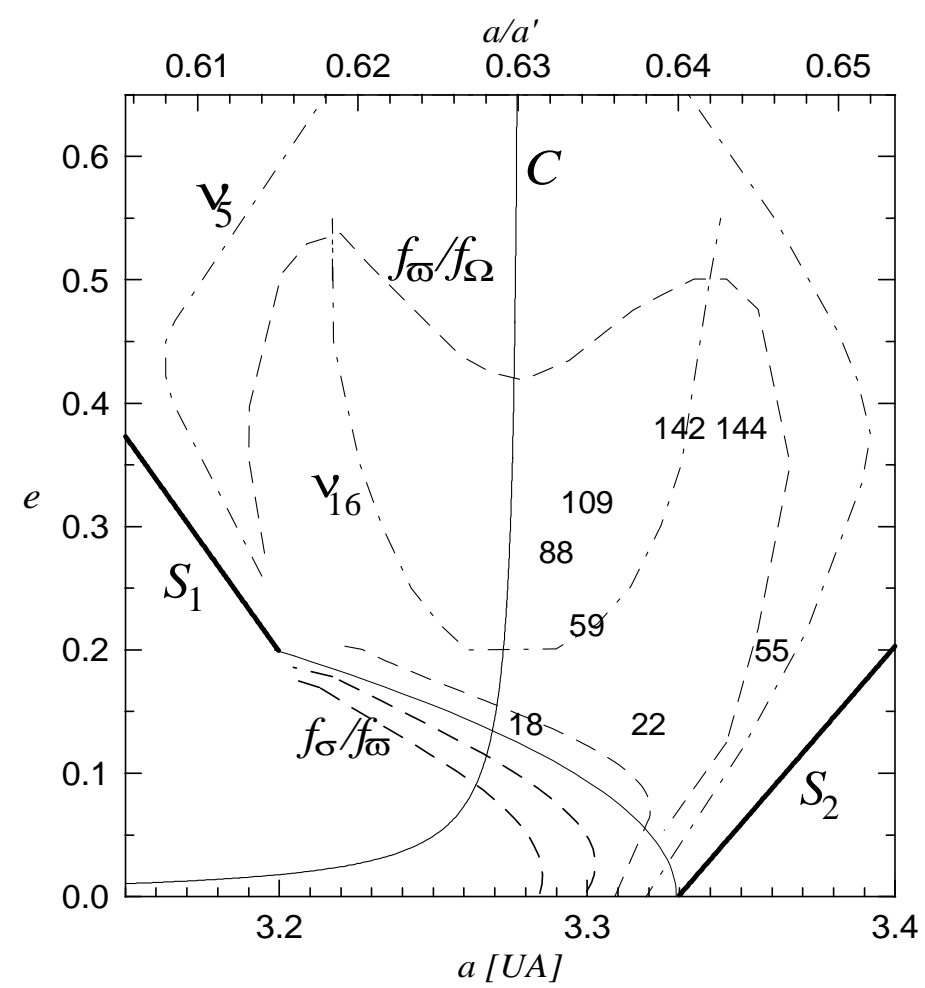

Figura 5.12: Posição das condições iniciais cujo comportamento é apresentado nas Figs. $5.13-5.26$. 
Visando exemplificar os diferentes comportamentos observados nos testes, apresentamos a seguir alguns resultados da evolução dinâmica de objetos, selecionados de forma a serem o mais representativos possível. Cada exemplo é identificado com um número, cuja posição inicial no espaço $a, e$ aparece na Fig. 5.12. Em todos os casos, mostramos 4 figuras (a,b,c,d) correspondentes às projeções da superfície de seção $\sigma=0^{\circ}, \omega=90^{\circ}$ sobre diferentes planos. Estas 4 projeções fornecem uma idéia bastante completa do comportamento do objeto. Nas figuras (a) as librações do ângulo crítico $\sigma$ podem ser avaliadas em função da posição das ressonâncias seculares e secundárias. As figuras (b) caracterizam muito bem o comportamento na terceira dimensão, vinculado em todos os casos estudados à circulação de $\omega$. As figuras (c) são similares às (a), mas mostram simultaneamente um conjunto de linhas que correspondem a diferentes níveis da energia do problema restrito médio. Estes níveis são dados pela interseção no plano $a, e$ entre a superfície de seção que definimos e as superfícies de Hamiltoniana $\mathcal{H}^{*}=$ cte. (cf. Eq. 3.17). Estas figuras substituem com muita vantagem aos gráficos de energia vs. tempo. Finalmente, as figuras (d) permitem caracterizar as variações da excentricidade em função do argumento $\varpi-\varpi^{\prime}$.

Na região das ressonâncias secundárias, o comportamento caracteriza-se por uma difusão importante na inclinação até $\sim 30^{\circ}$, e o posterior escape dos objetos quando $|\sigma|>90^{\circ}$ e $e>0.5$. As Figs. 5.13 e 5.14 mostram este comportamento. Isto está em muito bom acordo com os resultados já conhecidos sobre esta região. Na região próxima da separatriz os objetos também apresentam uma difusão importante em inclinação, provavelmente vinculada à presença da ressonância de Kozai. As Figs. 5.15 e 5.16 mostram este comportamento. Nestes casos o argumento $\varpi-\varpi^{\prime}$ libra em torno de zero, o que também está em bom acordo com o que conhecemos dessa região através de outros estudos.

Os objetos da região em torno do ramo pericêntrico para $e>0.2$ apresentam um comportamento curioso. Quando só levamos em conta as variações seculares, os asteróides mostram uma libração bastante regular de $\sigma$ e $e$ (Fig. 5.17). Quando adicionamos a freqüência $5 n_{\mathrm{S}}-2 n_{\mathrm{J}}$, a excentricidade começa a difundir até que o objeto escapa quando $e>0.65$ (Fig. 5.18).

A região de extrema regularidade para $\sigma \sim 80^{\circ}$ não mostra um comportamento muito interessante (Fig. 5.19). O espalhamento que acontece ao longo da linha de e- 
a.

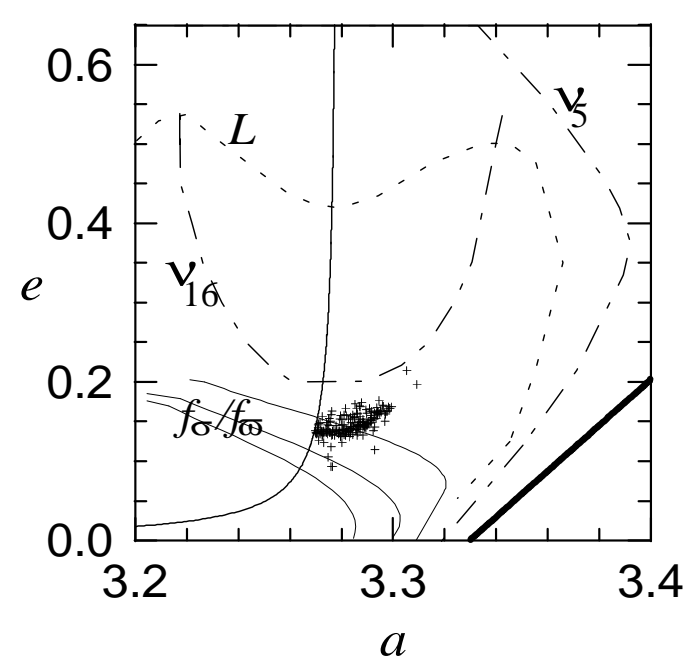

C.

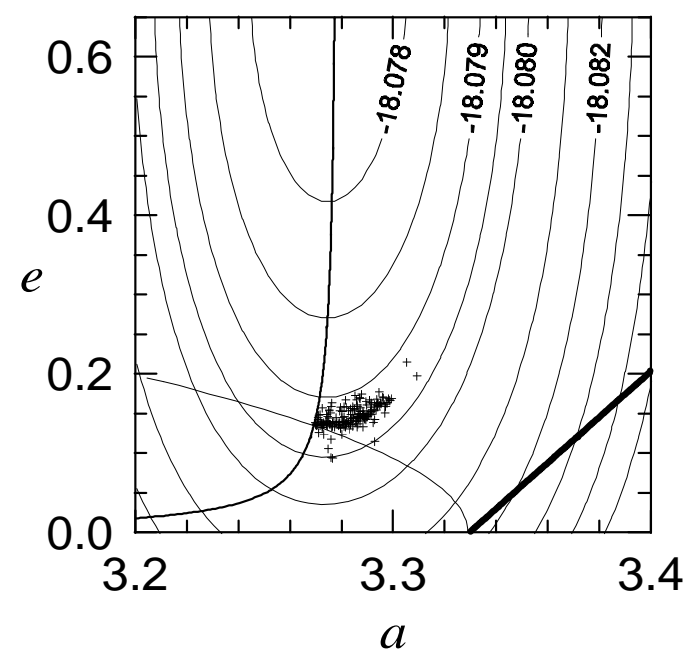

b.

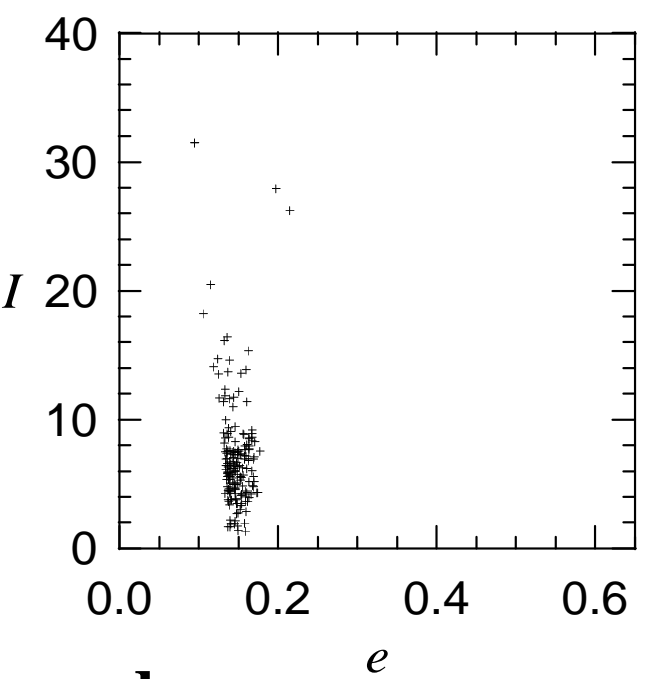

d.

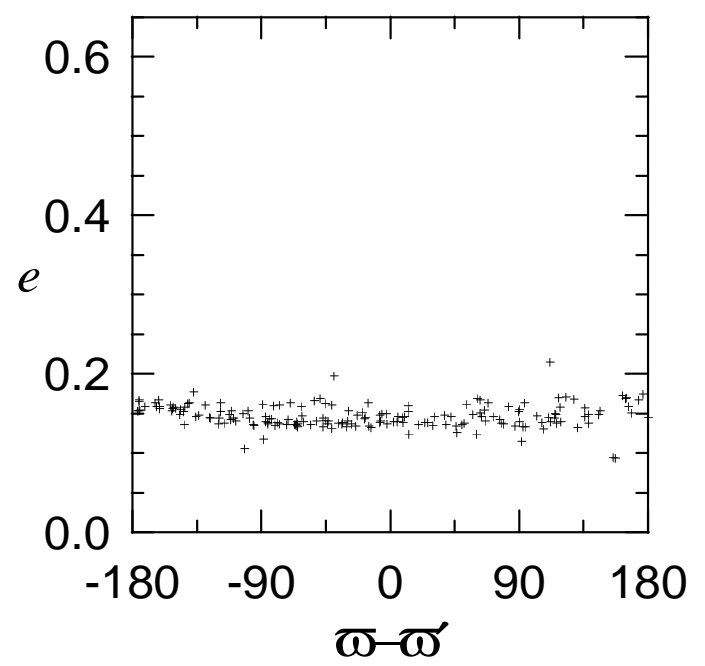

Figura 5.13: Condição inicial $\mathrm{N}^{\circ} 18$, no caso em que não levamos em conta a perturbação da comensurabilidade 5/2 entre Júpiter e Saturno. Os gráficos correspondem à superfície de seção $|\sigma|<1^{\circ},\left|\omega-90^{\circ}\right|<1^{\circ}$ : (a) Projeção no plano $a, e$ junto com algumas ressonâncias secundárias e seculares; (b) Projeção no plano e, $I$; (c) Igual que (a) mas agora mostrando diferentes níveis de $\mathcal{H}^{*}=$ cte.; (d) Projeção no plano $e, \varpi$. 

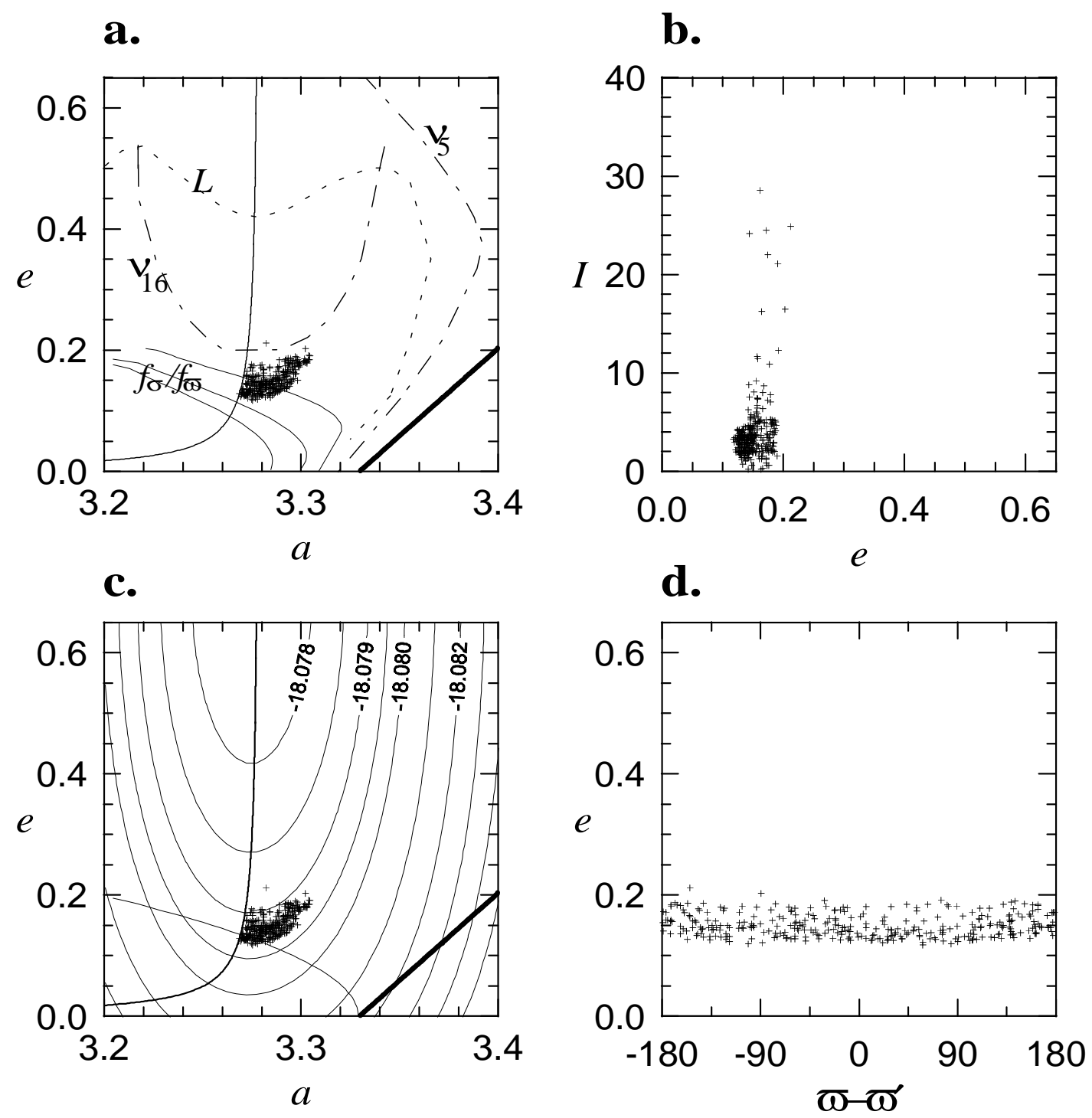

Figura 5.14: Condição inicial $N^{\circ} 18$, no caso em que $5 n_{\mathrm{S}}-2 n_{\mathrm{J}} \simeq 1470^{\prime \prime} /$ ano (valor real). 

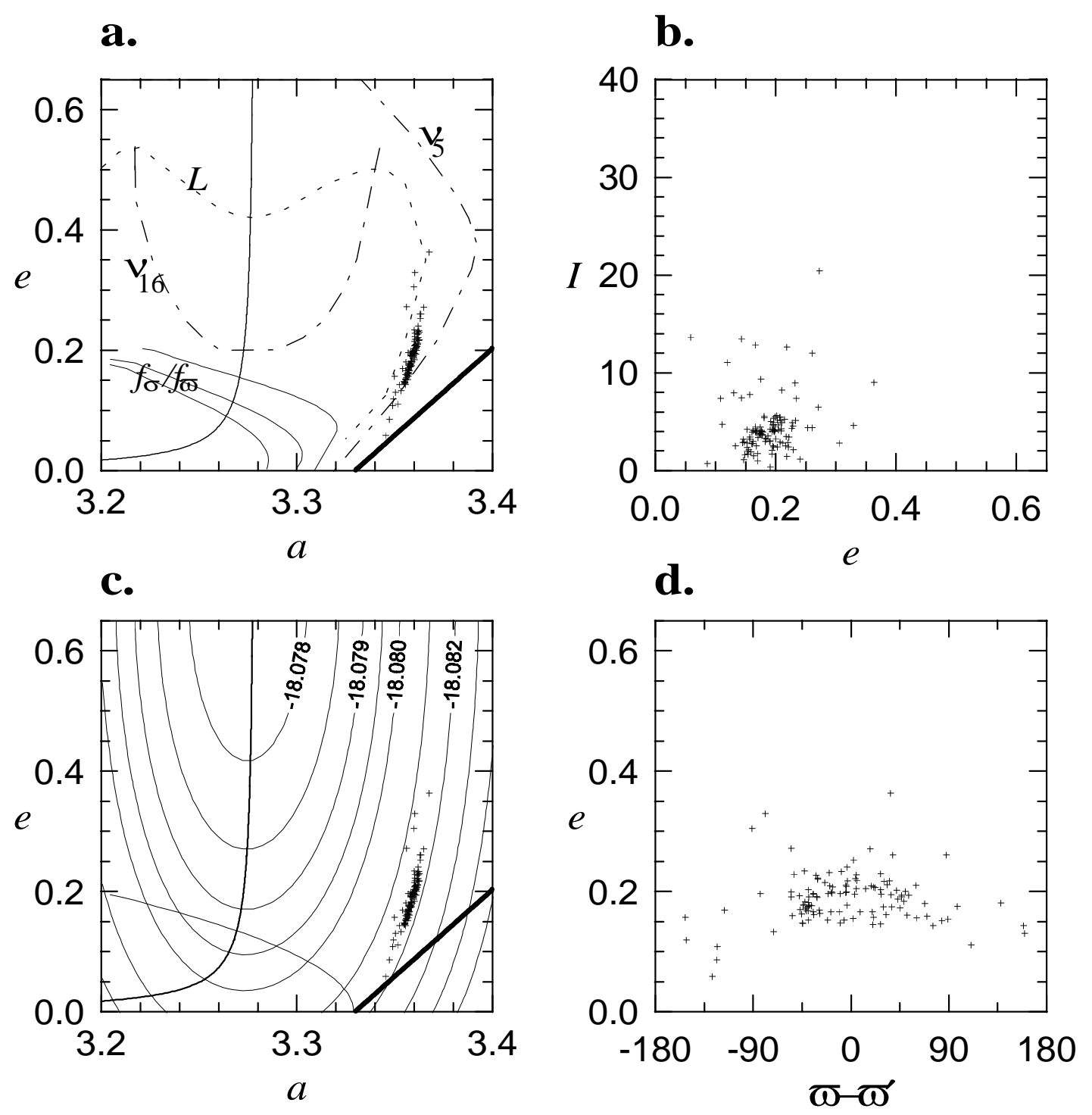

Figura 5.15: Condição inicial $N^{\circ} 55$, sem levar em conta a comensurabilidade $5 / 2$ entre Júpiter e Saturno. 

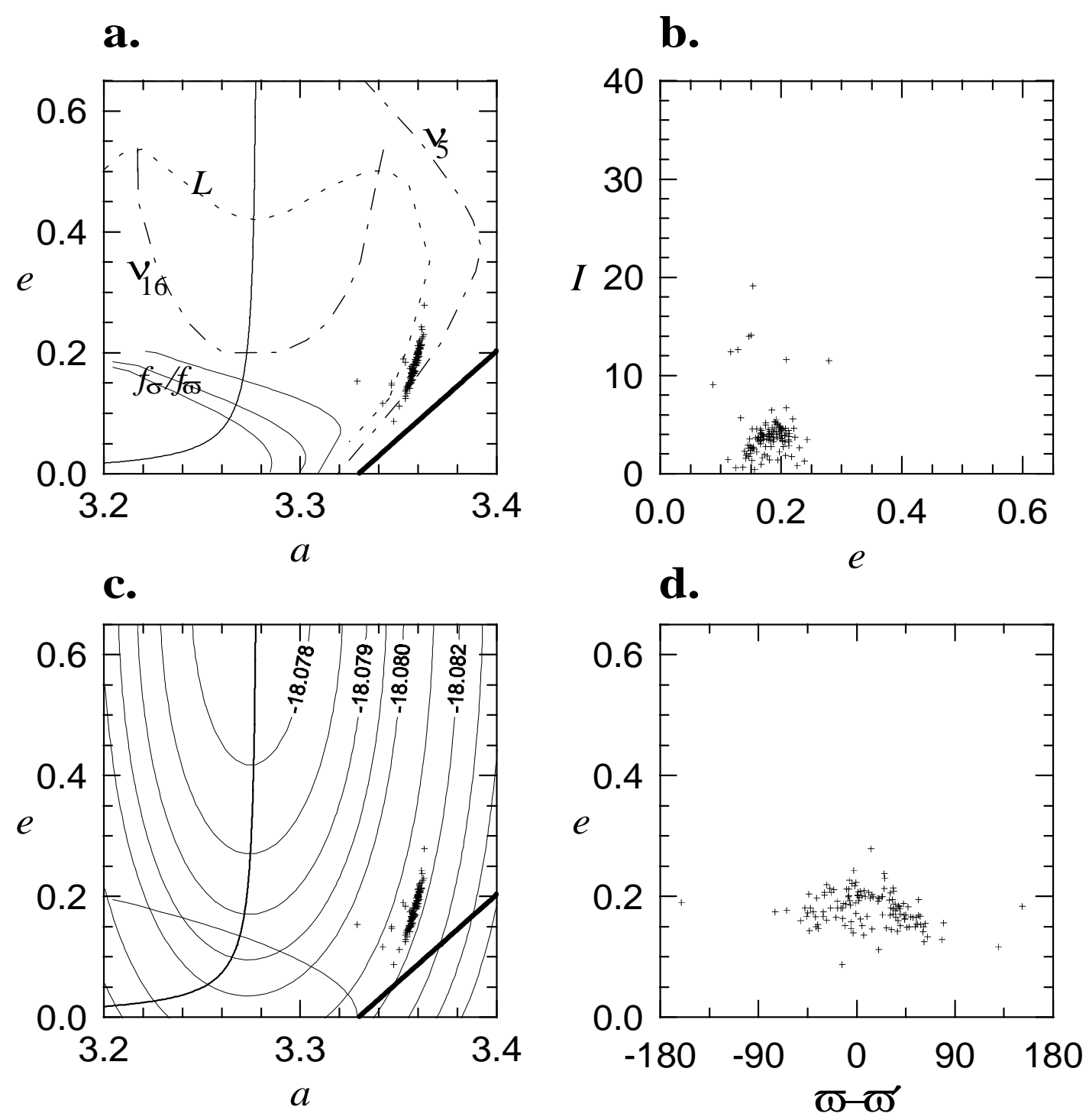

Figura 5.16: Condição inicial $N^{\circ} 55$, no caso em que $5 n_{\mathrm{S}}-2 n_{\mathrm{J}} \simeq 1470^{\prime \prime} /$ ano (valor real). 

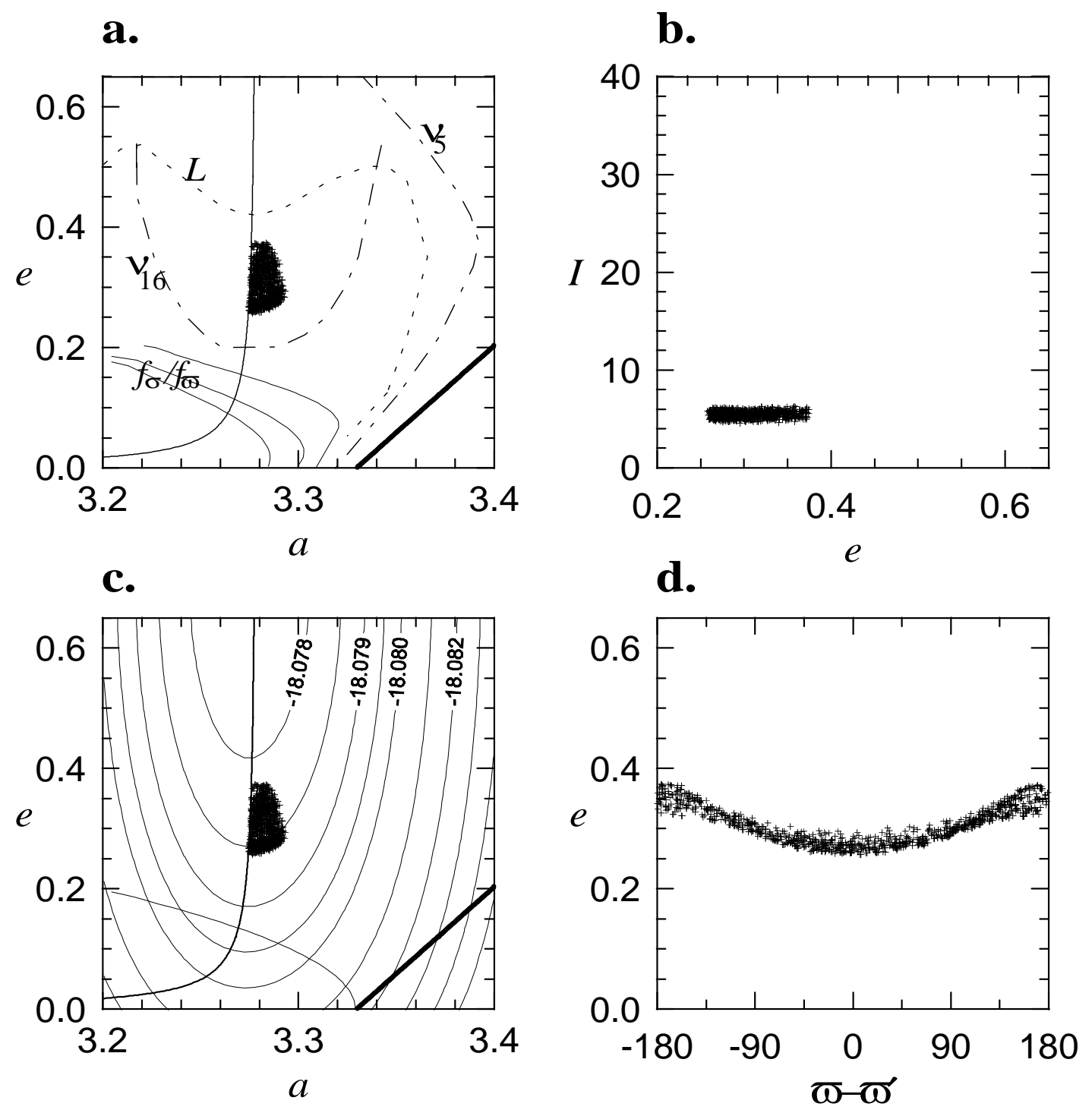

Figura 5.17: Condição inicial $N^{\circ} 88$, sem levar em conta a comensurabilidade $5 / 2$ entre Júpiter e Saturno. 

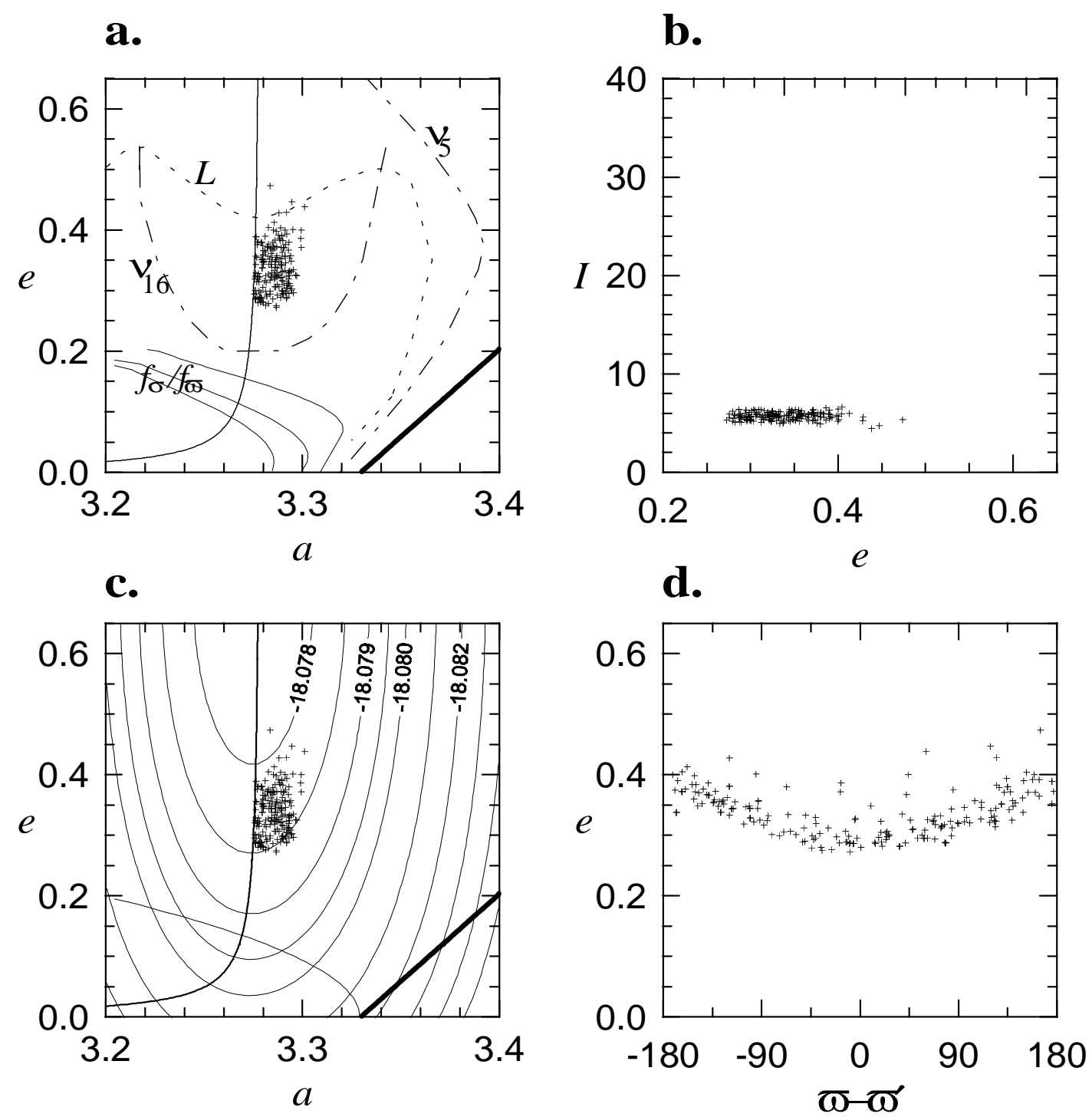

Figura 5.18: Condição inicial $N^{\circ} 88$, no caso em que $5 n_{\mathrm{S}}-2 n_{\mathrm{J}} \simeq 1470^{\prime \prime}$ /ano (valor real). 
nergia na Fig. 5.19c não é uma difusão, o que pode ser claramente verificado observando a Fig. 5.19d.

a.

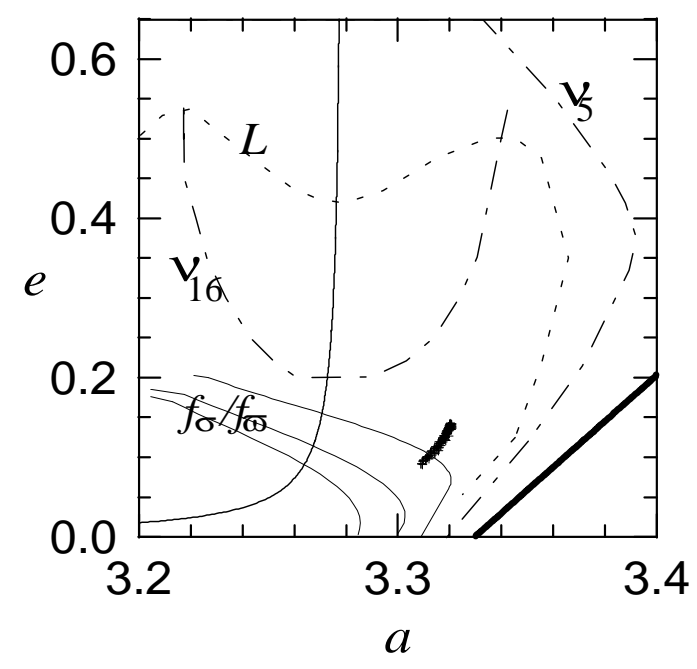

c.

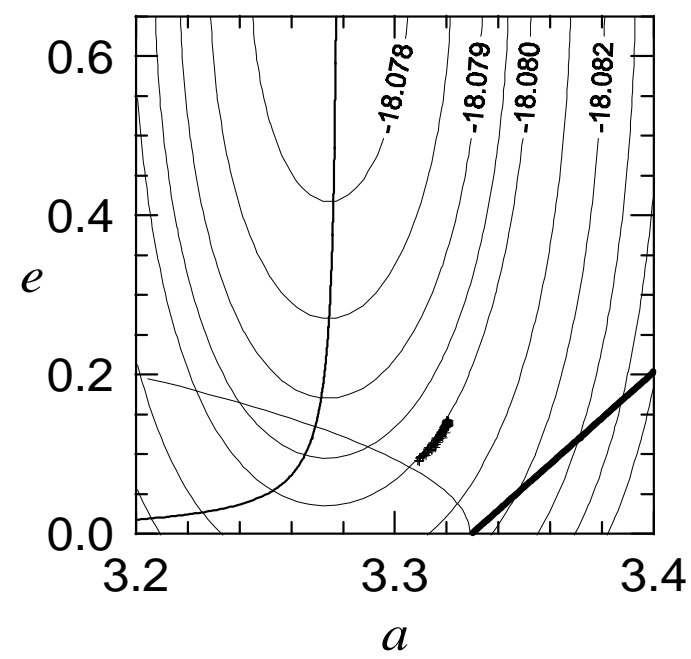

b.

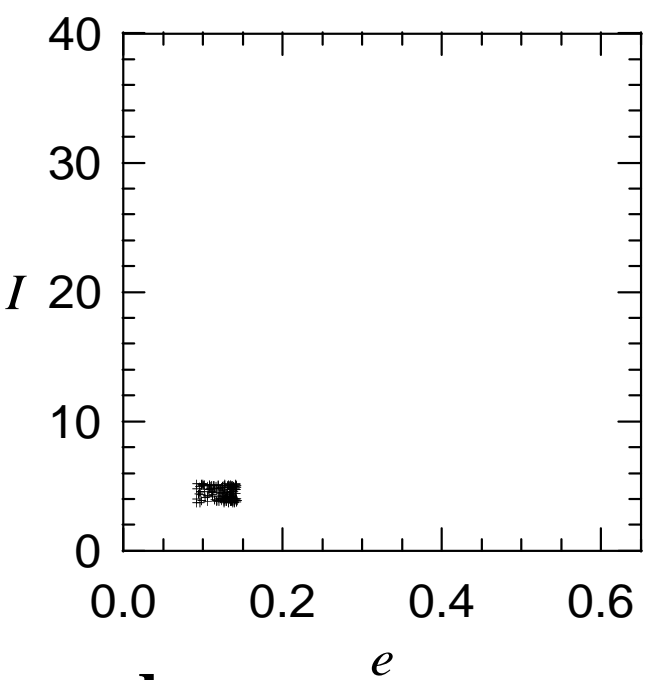

d.

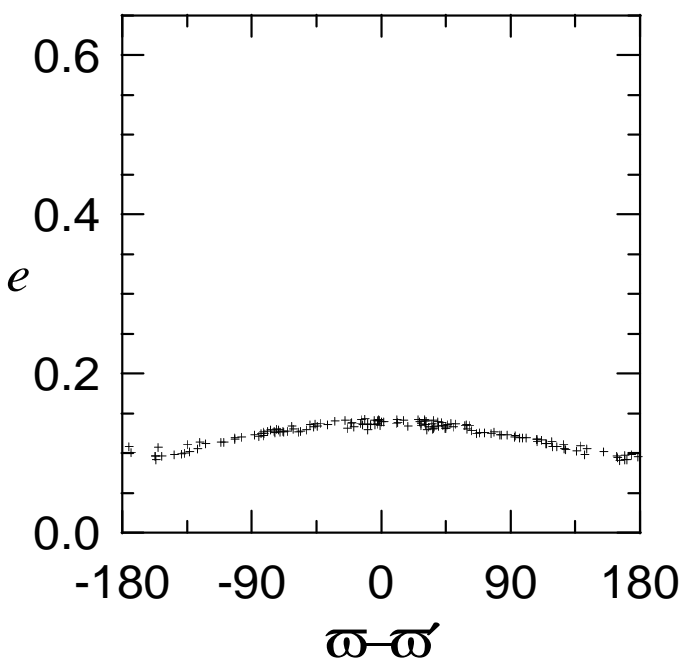

Figura 5.19: Condição inicial $N^{\circ} 22$, no caso em que $5 n_{\mathrm{S}}-2 n_{\mathrm{J}} \simeq 1470^{\prime \prime} /$ ano (valor real).

Ao contrario, objetos com comportamento muito interessante encontram-se sobre a ressonância secular $\nu_{16}$. Para $e<0.3$ (Fig. 5.20) os objetos mostram uma difusão notória na inclinação até que, em algum momento, a excentricidade cresce muito rapidamente (tanto que não dá para que o fenômeno chegue a ser percebido na superfície de seção) até valores de $e>0.65$; logo, o objeto escapa. Para $e>0.3$ (Fig. 5.21) a evolução é muito sensível à freqüência $5 n_{\mathrm{S}}-2 n_{\mathrm{J}}$, particularmente quando o valor desta 
é 5 vezes o valor real (Fig. 5.22). Neste último caso os objetos escapam, pois em algum momento $e>0.65$.

a.

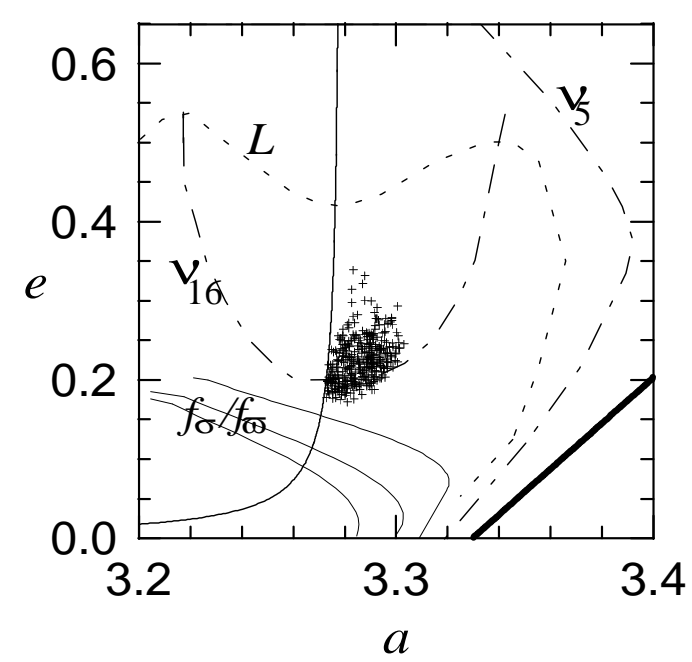

c.

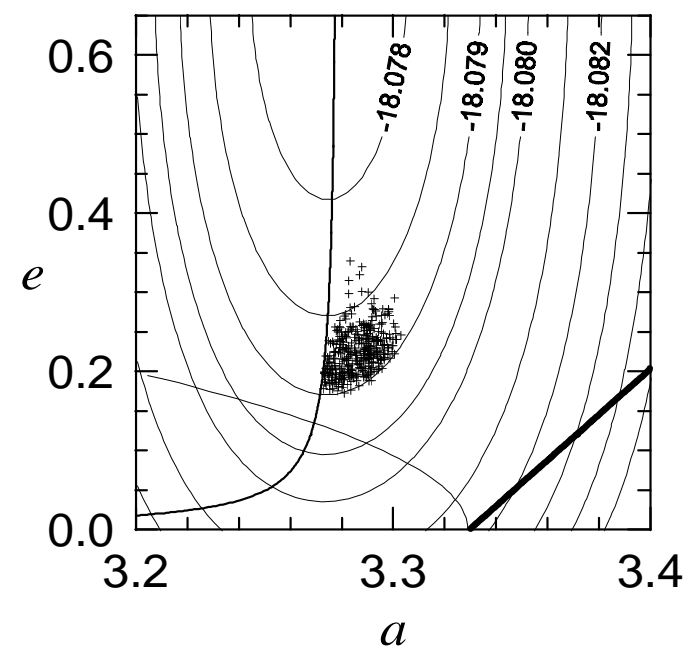

b.

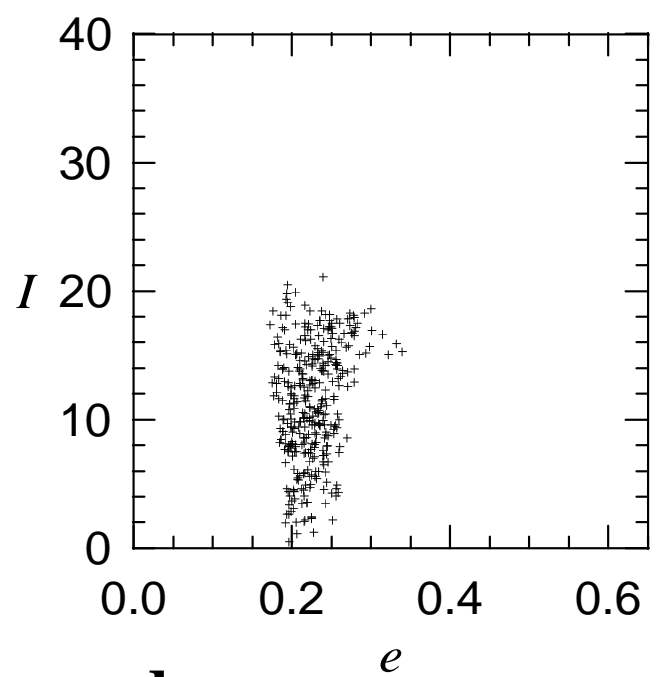

d.

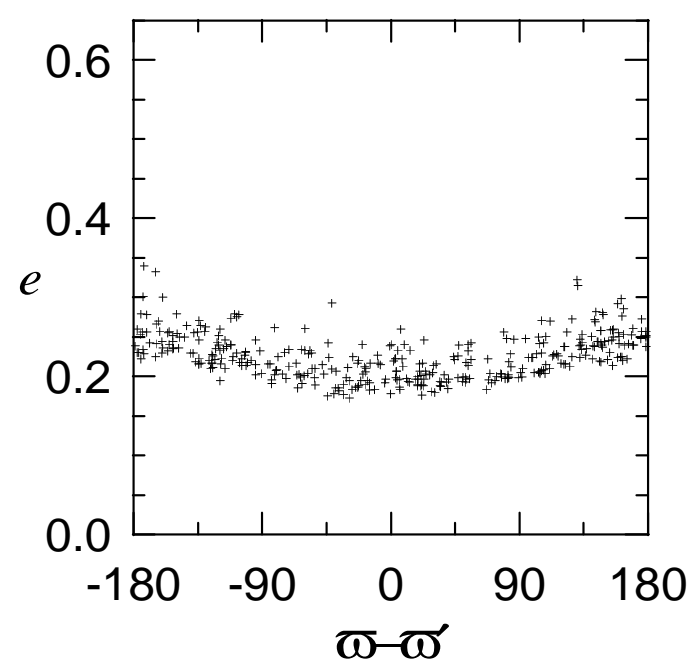

Figura 5.20: Condição inicial $N^{\circ} 59$, no caso em que $5 n_{\mathrm{S}}-2 n_{\mathrm{J}} \simeq 1470^{\prime \prime} /$ ano (valor real).

$\mathrm{Na}$ região compreendida entre o ramo pericêntrico e a $\nu_{16}$, mas longe do ramo pericêntrico, o comportamento é fortemente caótico quando o valor da freqüência $5 n_{\mathrm{S}}-$ $2 n_{\mathrm{J}}$ é 2 vezes o valor real. Isto pode ser claramente observado nas Figs. 5.23 e 5.24.

A outra região que é afetada notoriamente quando o valor de $5 n_{\mathrm{S}}-2 n_{\mathrm{J}}$ é 3 vezes o valor real, é a compreendida entre a $\nu_{16}$ e a borda $L$, para $e>0.3$. Nesse caso, o comportamento muito regular da Fig. 5.25 torna-se totalmente caótico na Fig. 5.26. 

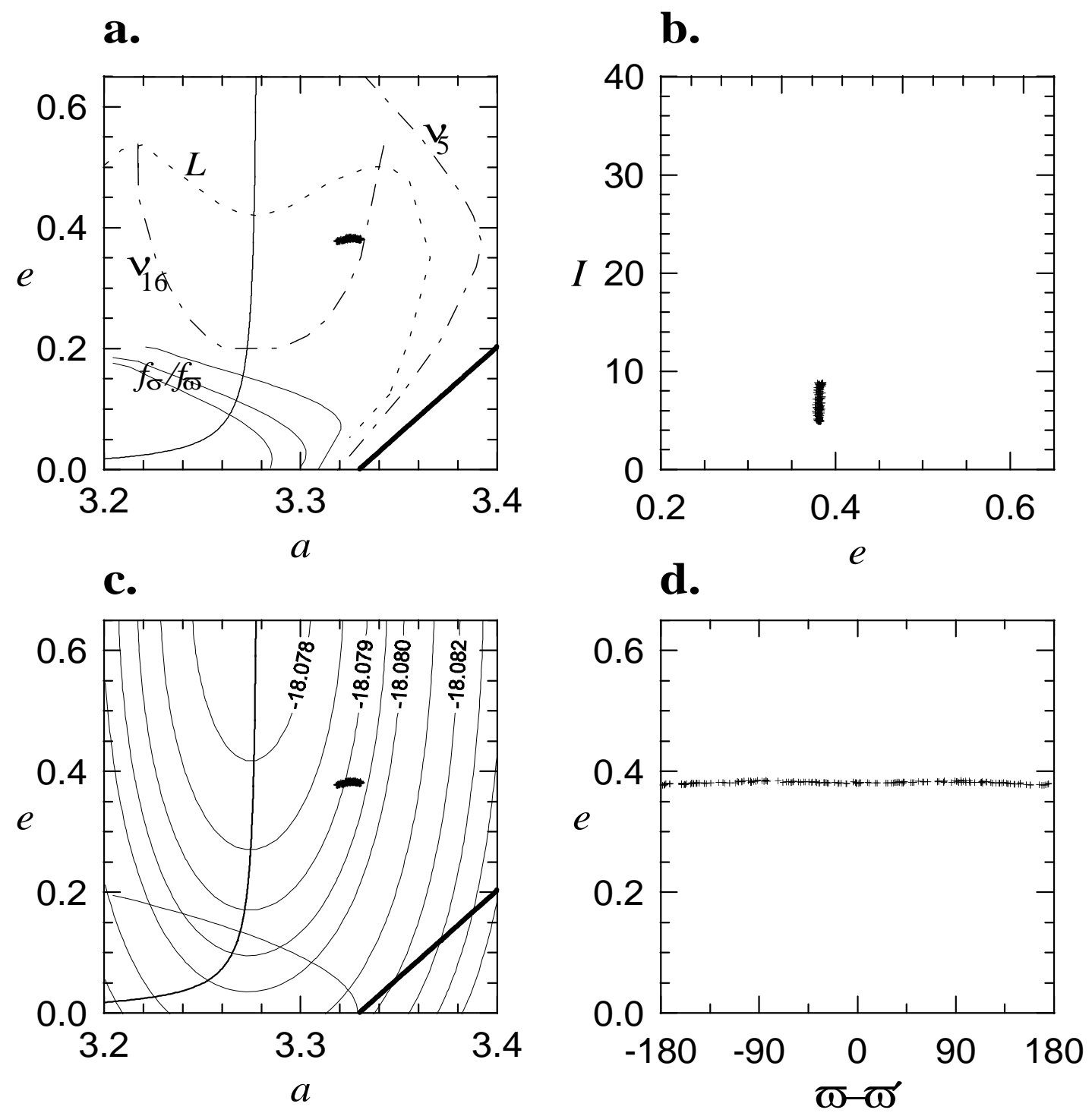

Figura 5.21: Condição inicial $N^{\circ} 142$, no caso em que $5 n_{\mathrm{S}}-2 n_{\mathrm{J}} \simeq 1470^{\prime \prime} /$ ano (valor real). 

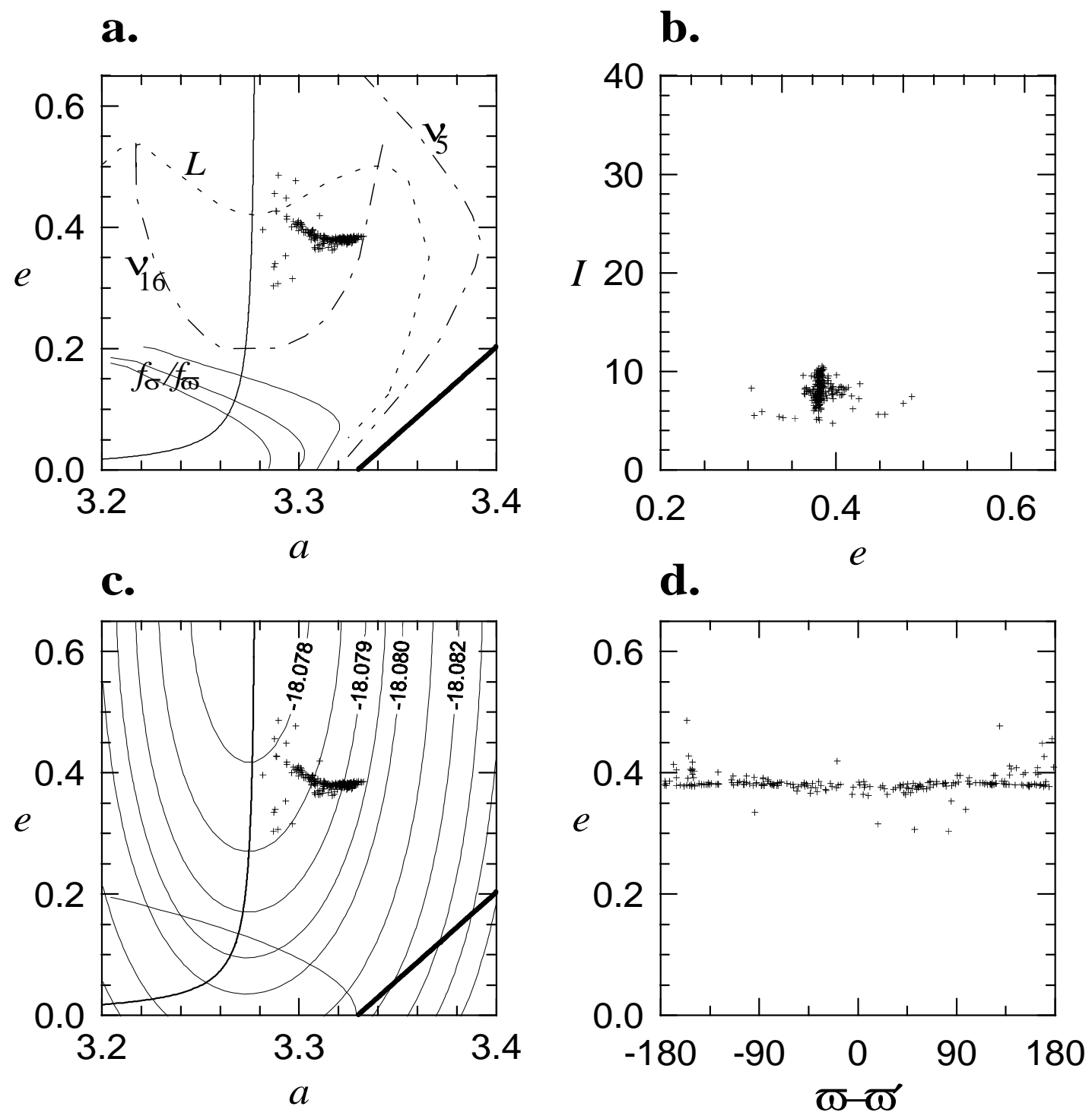

Figura 5.22: Condição inicial $N^{\circ} 142$, no caso em que $5 n_{\mathrm{S}}-2 n_{\mathrm{J}} \simeq 7350^{\prime \prime} /$ ano $(176$ anos de período). 
a.

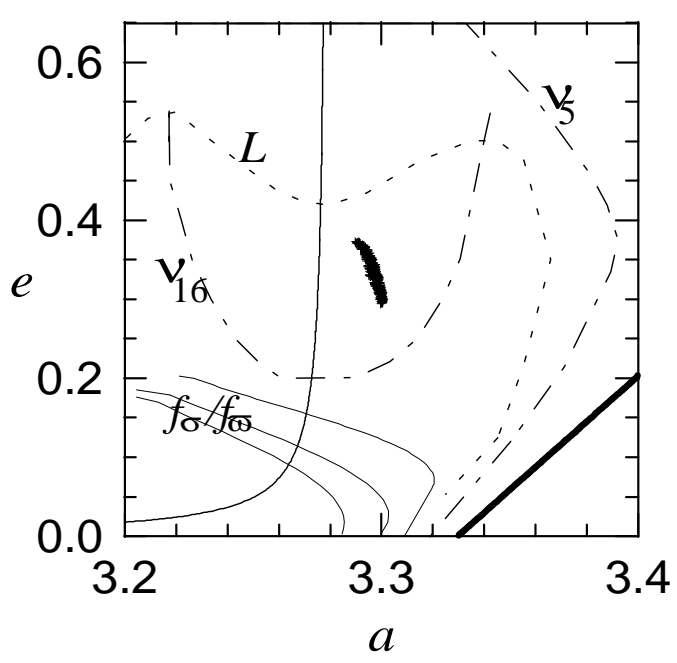

c.

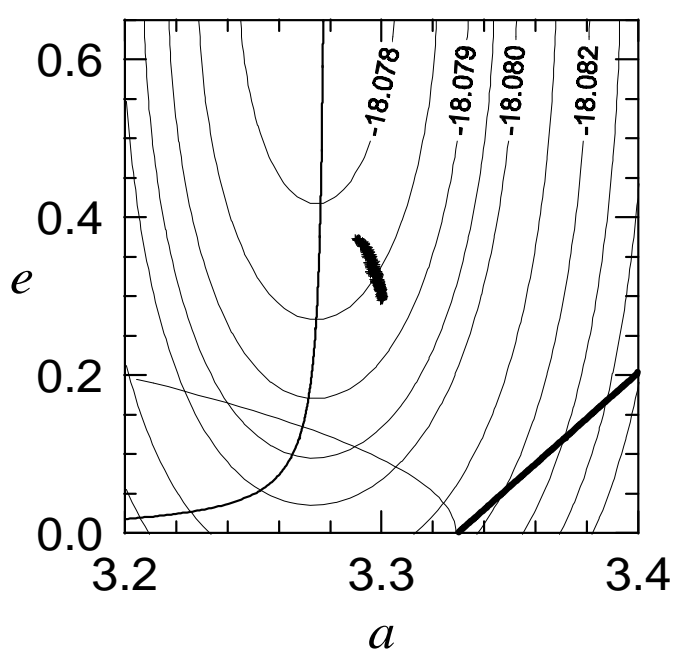

b.

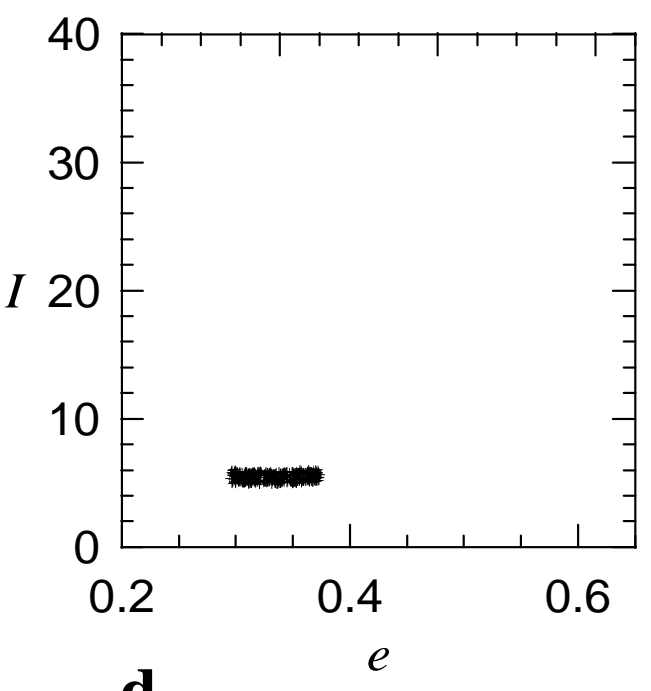

d.

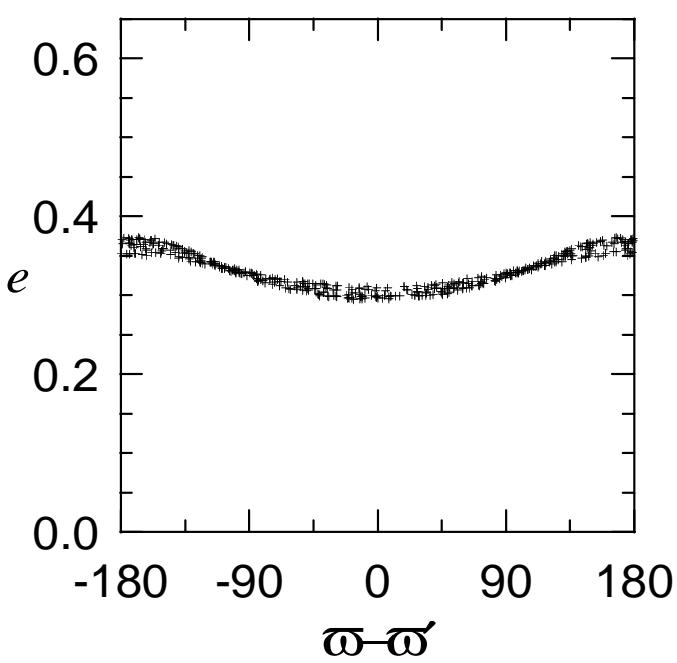

Figura 5.23: Condição inicial $N^{\circ} 109$, no caso em que $5 n_{\mathrm{S}}-2 n_{\mathrm{J}} \simeq 1470^{\prime \prime}$ /ano (valor real). 

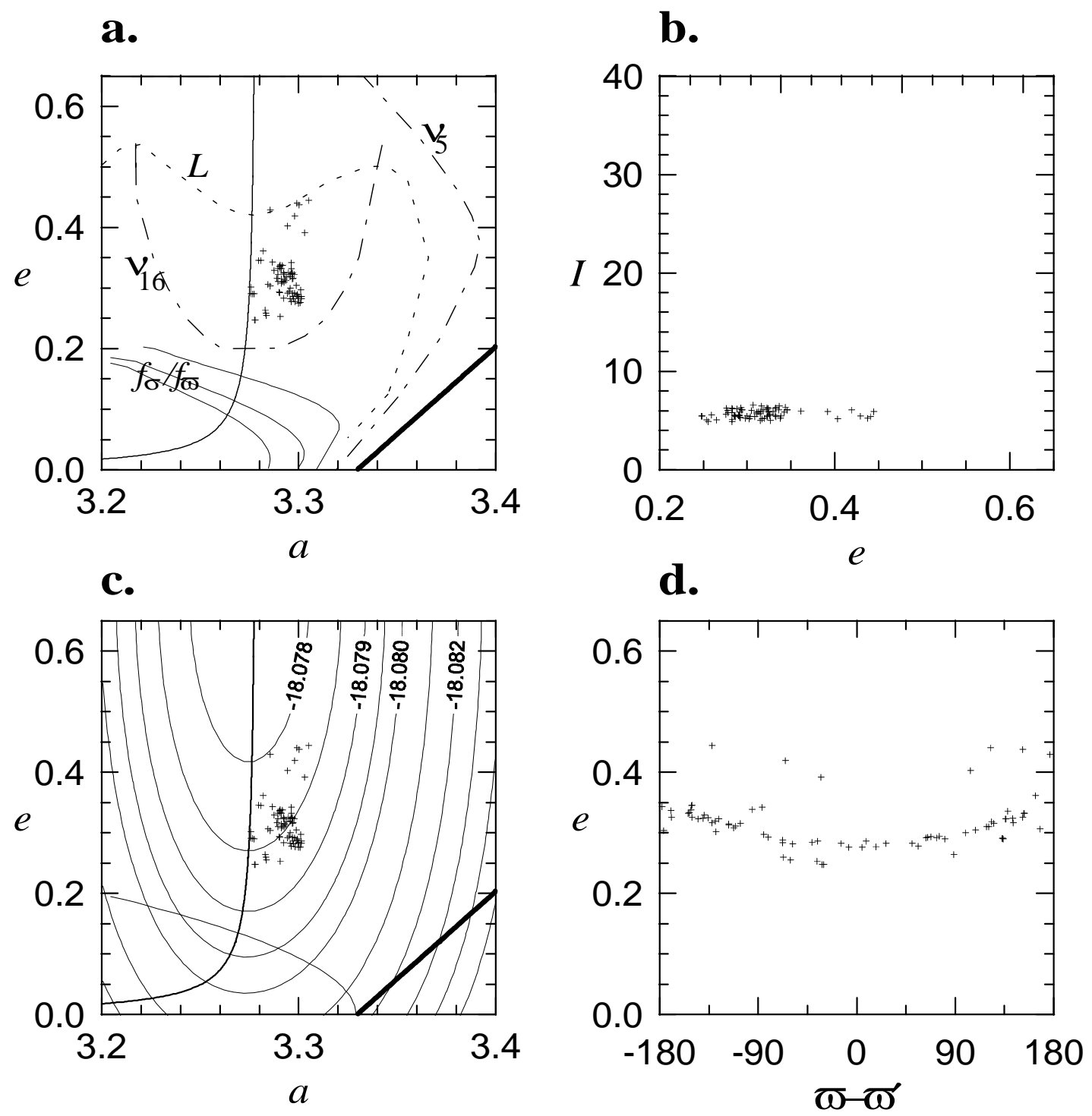

Figura 5.24: Condição inicial $\mathrm{N}^{\circ} 109$, no caso em que $5 n_{\mathrm{S}}-2 n_{\mathrm{J}} \simeq 2940$ " /ano (440 anos de período). 
O interessante é a rápida difusão na inclinação que leva a estes objetos de uma região de excentricidades altas até a região das circulações pericêntricas nas muito baixas excentricidades.

a.

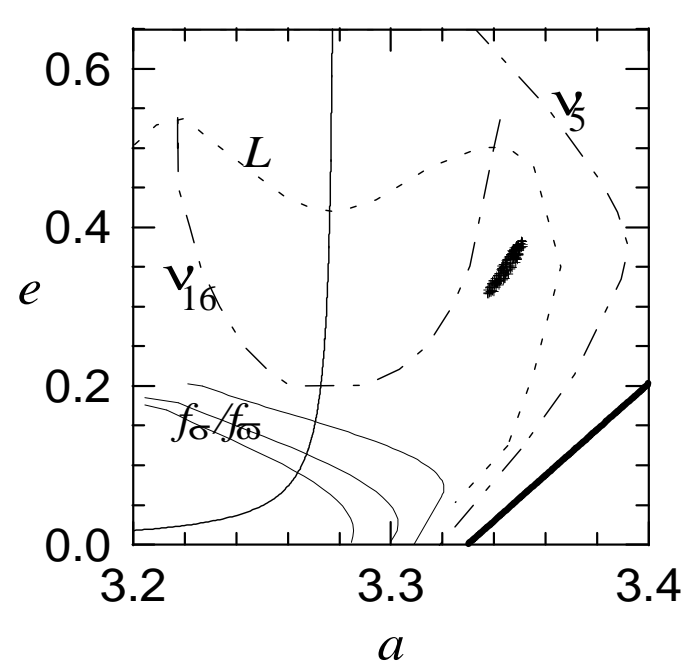

c.

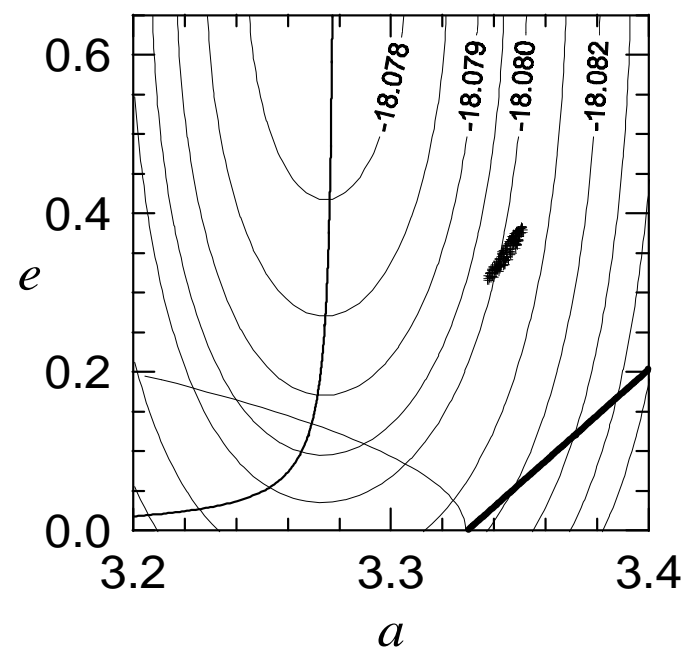

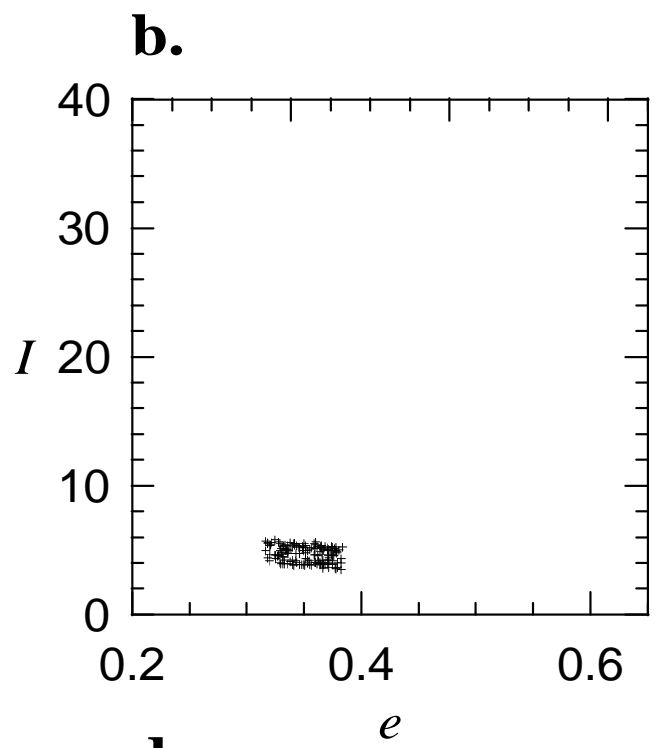

d.

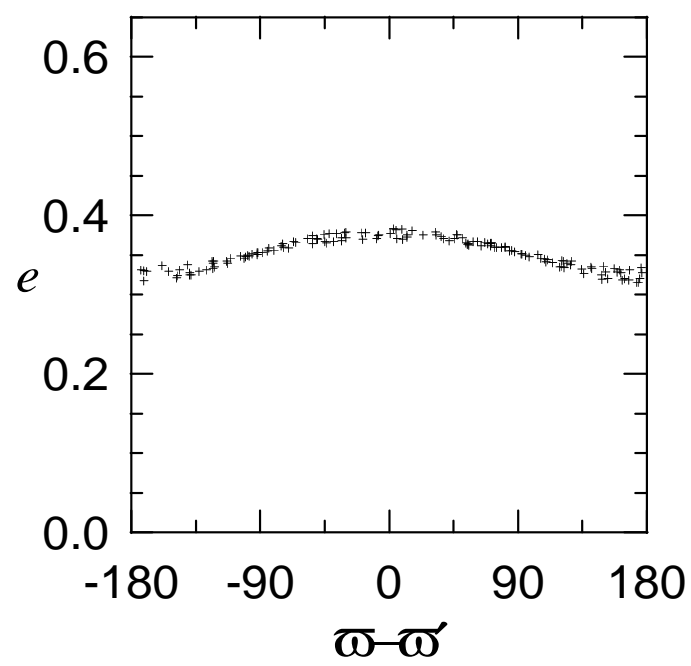

Figura 5.25: Condição inicial $\mathrm{N}^{\circ} 144$, no caso em que $5 n_{\mathrm{S}}-2 n_{\mathrm{J}} \simeq 1470^{\prime \prime} /$ ano (valor real).

Em termos gerais, podemos dizer que o mapeamento reproduz muito bem (qualitativamente) as principais características dinâmicas das diferentes regiões da ressonância 2/1. Em particular, é interessante fazer uma comparação visual entre as figuras (d) de $e$ vs. $\varpi-\varpi^{\prime}$ (nos casos em que consideramos o valor real de $5 n_{\mathrm{S}}-2 n_{\mathrm{J}}$ ), e as figuras que aparecem nos artigos de Lemaitre e Henrard (1990) e Morbidelli e Moons (1993), 
a.

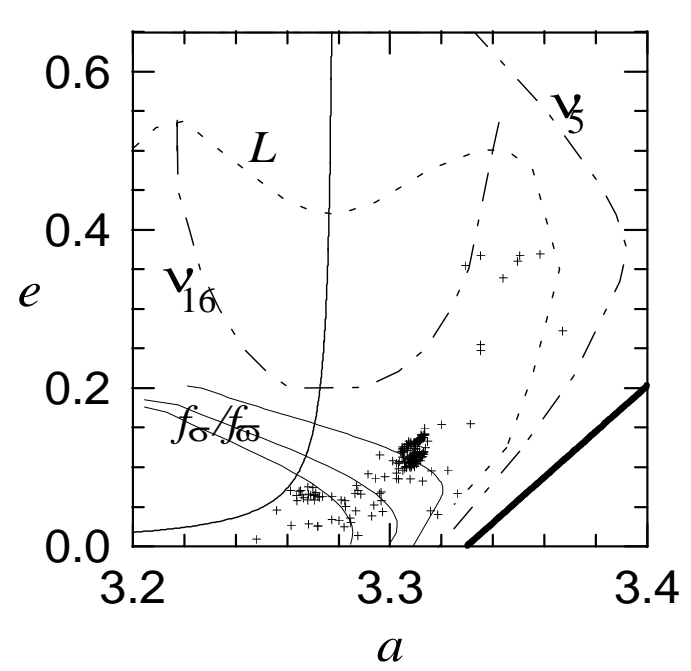

c.

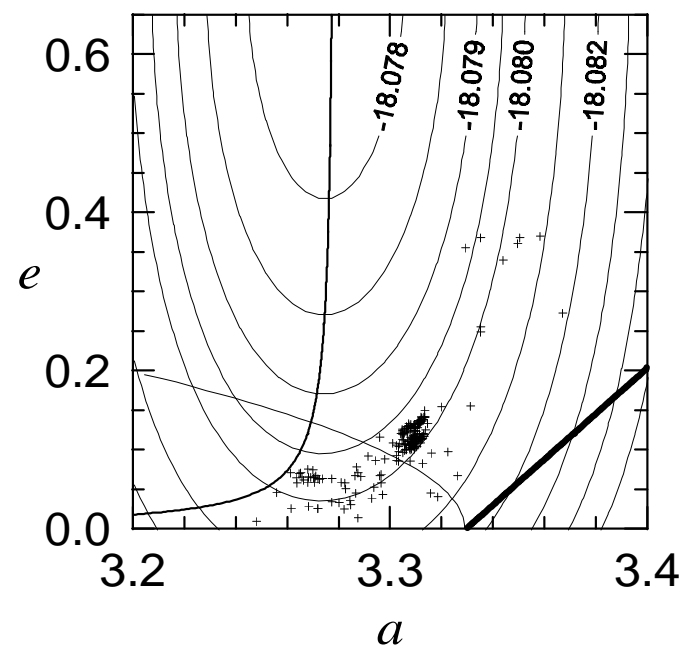

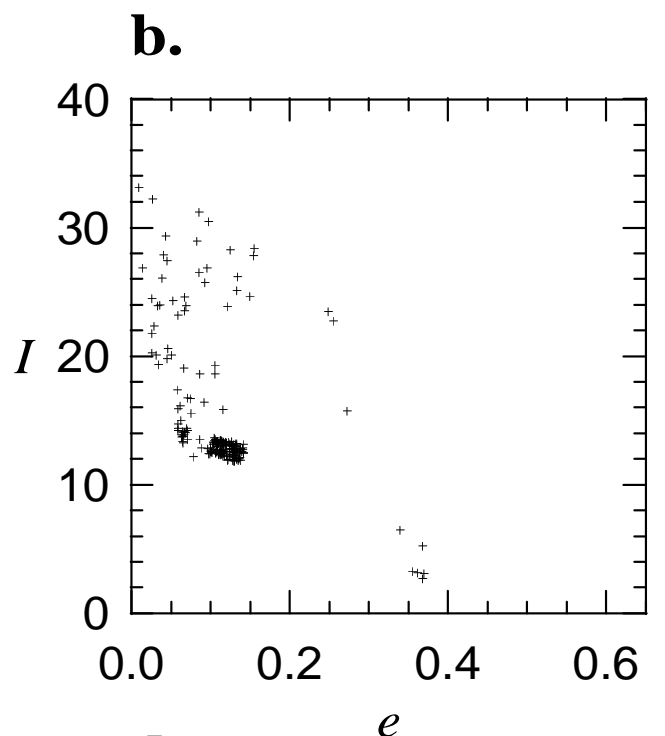

d.

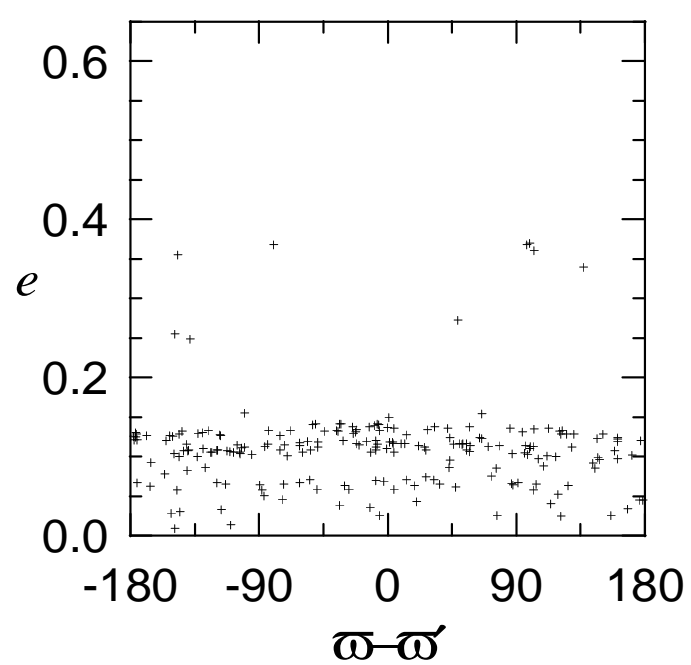

Figura 5.26: Condição inicial $\mathrm{N}^{\circ} 144$, no caso em que $5 n_{\mathrm{S}}-2 n_{\mathrm{J}} \simeq 4410^{\prime \prime} /$ ano $(293$ anos de período). 
e descobrir que existe uma grande similaridade entre elas.

\subsection{A ressonância $3 / 2$}

O estudo que fizemos da ressonância $3 / 2$ utilizando o mapeamento teve dois objetivos: por um lado, testar a confiabilidade do mapeamento verificando que os asteróides reais da ressonância não escapassem em intervalos de tempo da mesma ordem que os testados na ressonância 2/1. Por outro lado, tentar avaliar se a variação da freqüência $5 n_{\mathrm{S}}-2 n_{\mathrm{J}}$ afeta sensivelmente a dinâmica destes objetos reais.

A Fig. 5.27 mostra a distribuição nos planos $a, e$ e $e, I$ de 82 asteróides reais na ressonância 3/2. Estes asteróides foram escolhidos da base de dados de Bowell et al. (1994), e são listados no Apêndice B. Para montar a Fig. 5.27, as condições iniciais dos objetos fornecidas pela base de dados foram integradas utilizando o código RA15 (Everhart, 1985) com um passo constante de 10 dias, até a condição simultânea

$$
\left|3 \lambda^{\prime}-2 \lambda-\varpi\right| \leq 4^{\circ}, \quad\left|\varpi-\varpi^{\prime}\right| \leq 4^{\circ}, \quad\left|\omega-90^{\circ}\right| \leq 4^{\circ}
$$

Como podemos observar, a maior parte dos objetos agrupam-se numa região bem definida em torno do ramo pericêntrico, abaixo da borda inferior das separatrizes das ressonâncias seculares (curva $L$ ), e com amplitudes de libração moderadas. Só alguns poucos objetos, ocupam a região das circulações pericêntricas nas excentricidades muito baixas, e a região das ressonâncias seculares por fora da borda $L$.

As condições iniciais destes asteróides são apresentadas no Apêndice A, e foram integradas com o mapeamento por um intervalo de tempo de $10^{8}$ anos. Estudamos o que acontecia levando-se em conta ou não as perturbações da comensurabilidade $5 / 2$ na órbita de Júpiter. Também fizemos testes utilizando 7 valores diferentes da freqüência $5 n_{\mathrm{S}}-2 n_{\mathrm{J}}$.

O resultado principal foi que quase todos os objetos permaneceram na ressonância durante todo o tempo de integração e só uns poucos escaparam. Este comportamento repetiu-se em todos testes que fizemos, sendo que os objetos que escaparam em cada teste foram quase sempre os mesmos. Os escapes foram avaliados de forma similar à aplicada na ressonância 2/1, controlando que:

1. a excentricidade fosse $e>0.45$ 

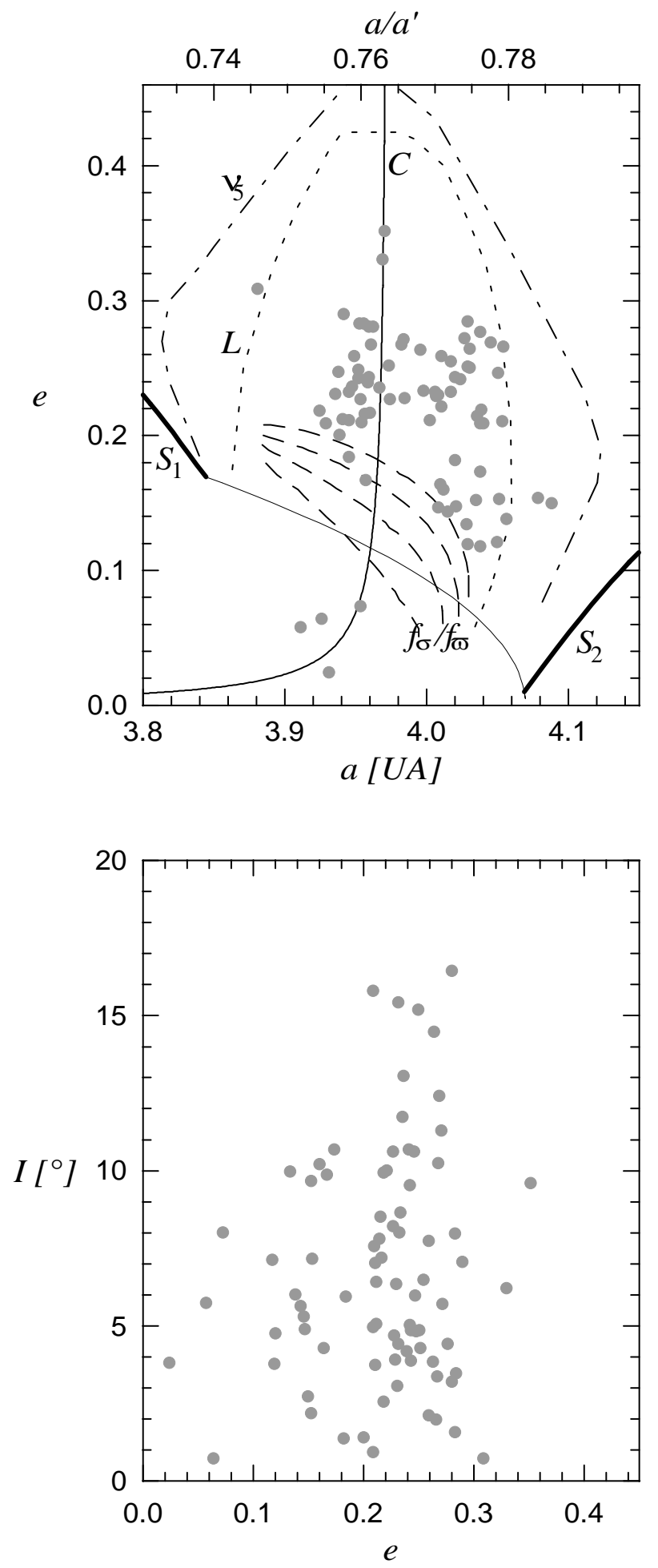

Figura 5.27: Ressonância 3/2. Posição sobre a superfície de seção Eq. (5.3) dos 82 asteróides numerados e multi-oposicionais listados na Tabela I do Apêndice A. Projeções nos planos $a, e$ e $e, I$. 
2. $|\sigma|>120^{\circ}$ e $e>0.35$ em forma simultânea, ficando assim perto da curva de colisão

3. a inclinação fosse $I>40^{\circ}$

Os objetos que escaparam em cada um dos testes são listados na Tabela 5.2, onde também indicamos se o escape aconteceu antes ou depois de $10^{6}$ anos. Destaquemos que, dentre todos estes escapes, muito poucos (apenas 6\%) corresponderam à condição $e>0.45$, e particularmente, nenhum deles correspondeu à condição $I>40^{\circ}$, que não foi observada nos testes realizados. De fato, alguns destes objetos possuem órbitas regulares, porém sua amplitude de libração é muito grande e não podem ser integrados com o nosso mapeamento. A palavra "escape" utilizada neste contexto refere-se às três condições estabelecidas acima, e nem sempre significa que o objeto escapa como conseqüência de um comportamento caótico.

Nas Figs. 5.28 e 5.29 indicamos a posição inicial dos objetos que escaparam, utilizando círculos e cruzes para distinguir se o escape aconteceu antes ou depois de $10^{6}$ anos, respectivamente. A Fig. 5.28 corresponde ao caso em que consideramos o valor real de $5 n_{\mathrm{S}}-2 n_{\mathrm{J}}$, e a Fig. 5.29 ao caso em que $5 n_{\mathrm{S}}-2 n_{\mathrm{J}}$ é igual a 3.2 vezes o valor real. Neste último caso, o período da comensurabilidade $5 / 2$ fica por volta de 273 anos. Este valor coincide, aproximadamente, com o período de libração do argumento crítico $\sigma$, que pode flutuar entre 270 anos (para amplitudes de libração pequenas) e 220 anos (para amplitudes de libração moderadas). É interessante mencionar que, tanto na Fig. 5.28 quanto na Fig. 5.29, os 4 objetos na região de circulação pericêntrica (grupo de 334 Chicago) "escaparam" muito rapidamente (menos de 10000 anos) e não foi possível nesses casos construir as superfícies de seção ${ }^{2}$. Por sua vez, vários objetos na região em torno do ramo pericêntrico são sensivelmente afetados pela mudança no valor da freqüência $5 n_{\mathrm{S}}-2 n_{\mathrm{J}}$.

O histograma da Fig. 5.30 é similar ao da Fig. 5.10. Podemos ver que o número de escapes aumenta subitamente quando há batimento entre $5 n_{\mathrm{S}}-2 n_{\mathrm{J}}$ e a freqüência de libração de $\sigma$. Porém, o número de escapes observados é muito pequeno, e este resultado não é tão estatisticamente significativo quanto no caso da ressonância $2 / 1$. Talvez, o

\footnotetext{
${ }^{2}$ Em particular, estes objetos possuem excentricidades muito baixas que fazem com que em algum momento da integração, as equações do mapeamento se tornem singulares (cumpre-se a condição estabelecida pela Eq. (3.49)).
} 


\begin{tabular}{|c|c|c|c|c|c|c|c|c|c|}
\hline \multicolumn{2}{|r|}{ Asteróide } & \multicolumn{7}{|c|}{ Período ressonância 5/2 [anos] } & \multirow{2}{*}{ Sem $5 / 2$} \\
\hline & & 1173 & 880 & 440 & 273 & 220 & 176 & 126 & \\
\hline 334 & Chicago & $\mathrm{x}$ & $\mathrm{x}$ & $\mathrm{x}$ & $\mathrm{x}$ & $\mathrm{x}$ & $\mathrm{x}$ & $\mathrm{x}$ & $\mathrm{x}$ \\
\hline 361 & Bononia & & & & $\mathrm{xx}$ & & & & \\
\hline 1038 & Tuckia & $\mathrm{xx}$ & & & $\mathrm{xx}$ & & & & \\
\hline 1202 & Marina & & & & $\mathrm{x}$ & & & & \\
\hline 1256 & Normania & $\mathrm{x}$ & $\mathrm{x}$ & $\mathrm{x}$ & $\mathrm{x}$ & $\mathrm{x}$ & $\mathrm{x}$ & $\mathrm{x}$ & $\mathrm{x}$ \\
\hline 1269 & Rollandia & $\mathrm{x}$ & $\mathrm{x}$ & $\mathrm{x}$ & $\mathrm{x}$ & $\mathrm{x}$ & $\mathrm{x}$ & $\mathrm{x}$ & $\mathrm{x}$ \\
\hline 1529 & Oterma & $\mathrm{xx}$ & $\mathrm{xx}$ & & $\mathrm{xx}$ & & & $\mathrm{xx}$ & $\mathrm{xx}$ \\
\hline 1754 & Cunningham & & & & $\mathrm{xx}$ & & & $\mathrm{xx}$ & $\mathrm{xx}$ \\
\hline 1877 & Marsden & & & & $\mathrm{xx}$ & & & & \\
\hline 2760 & Kacha & $\mathrm{xx}$ & $\mathrm{xx}$ & & & $\mathrm{xx}$ & & $\mathrm{xx}$ & $\mathrm{xx}$ \\
\hline 3202 & Graff & & & $\mathrm{xx}$ & $\mathrm{xx}$ & & $\mathrm{xx}$ & $\mathrm{xx}$ & $\mathrm{xx}$ \\
\hline 3557 & Sokolsky & $\mathrm{xx}$ & $\mathrm{xx}$ & $\mathrm{xx}$ & $\mathrm{xx}$ & $\mathrm{xx}$ & $\mathrm{xx}$ & $\mathrm{xx}$ & $\mathrm{xx}$ \\
\hline 3571 & Milanstefanik & & & & $\mathrm{xx}$ & & & & \\
\hline 3655 & Eupraksia & $\mathrm{x}$ & $\mathrm{x}$ & $\mathrm{x}$ & $\mathrm{x}$ & $\mathrm{x}$ & $\mathrm{x}$ & $\mathrm{x}$ & $\mathrm{x}$ \\
\hline 4196 & Shuya & $\mathrm{x}$ & $\mathrm{x}$ & $\mathrm{x}$ & $\mathrm{x}$ & $\mathrm{x}$ & $\mathrm{x}$ & $\mathrm{x}$ & $\mathrm{x}$ \\
\hline 5439 & $1990 \mathrm{RW}$ & & & & & & $\mathrm{x}$ & & \\
\hline 6984 & $1994 \mathrm{AO}$ & & & & $\mathrm{xx}$ & & & & \\
\hline 7174 & $1988 \mathrm{SQ}$ & $\mathrm{xx}$ & $\mathrm{xx}$ & $\mathrm{xx}$ & $\mathrm{xx}$ & $\mathrm{xx}$ & $\mathrm{xx}$ & $\mathrm{xx}$ & $\mathrm{xx}$ \\
\hline 7394 & 1985 QX4 & $\mathrm{x}$ & $\mathrm{x}$ & $\mathrm{x}$ & $\mathrm{x}$ & $\mathrm{x}$ & $\mathrm{x}$ & $\mathrm{x}$ & $\mathrm{x}$ \\
\hline \multirow[t]{3}{*}{7458} & 1984 DE1 & $\mathrm{x}$ & $\mathrm{x}$ & $\mathrm{x}$ & $\mathrm{x}$ & $\mathrm{x}$ & $\mathrm{x}$ & $\mathrm{x}$ & $\mathrm{x}$ \\
\hline & 1995 YK3 & & $\mathrm{x}$ & $\mathrm{x}$ & $\mathrm{x}$ & $\mathrm{x}$ & & $\mathrm{x}$ & $\mathrm{x}$ \\
\hline & 4216 T-2 & & & & & & $\mathrm{xx}$ & & \\
\hline
\end{tabular}

Tabela 5.2: Lista dos asteróides da ressonância $3 / 2$ que "escaparam" durante a integração, em função do valor do período do termo $5 \lambda_{\mathrm{S}}-2 \lambda_{\mathrm{J}}$. Aqueles indicados com (x) têm um tempo de escape $T<10^{6}$ anos e os indicados com (xx) têm um tempo de escape $10^{6}<T<10^{8}$. 

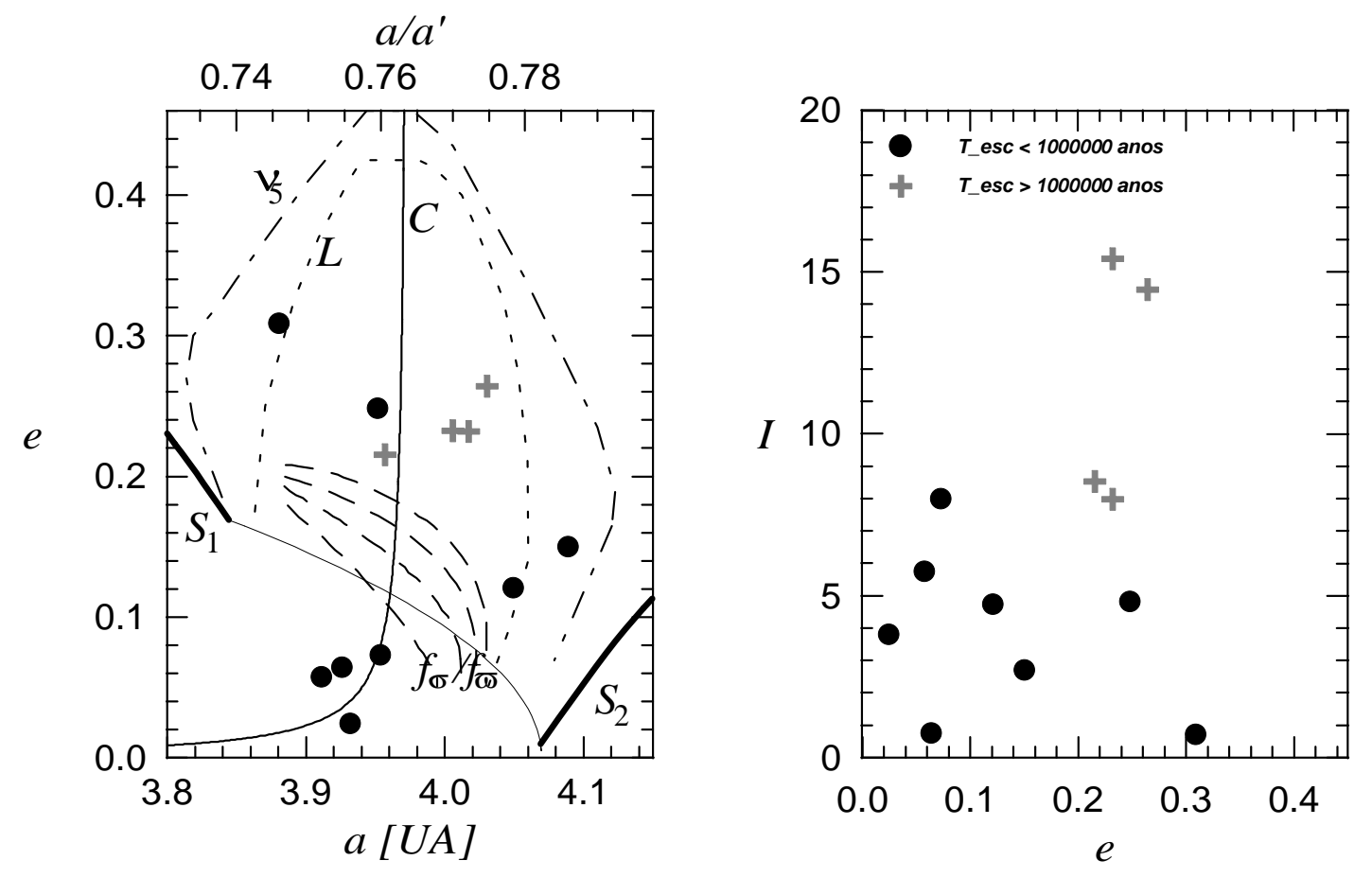

Figura 5.28: Posição inicial dos objetos que escaparam durante a integração, no caso em que consideramos $5 n_{\mathrm{S}}-2 n_{\mathrm{J}} \simeq 1470^{\prime \prime} /$ ano (valor real). Os círculos pretos representam objetos que escapam em $T<10^{6}$ anos. As cruzes são objetos que escapam em $T>10^{6}$ anos. Os asteróides que aparecem na região das circulações pericêntricas são Chicago, Normania, Shuya e 1985QX4. 

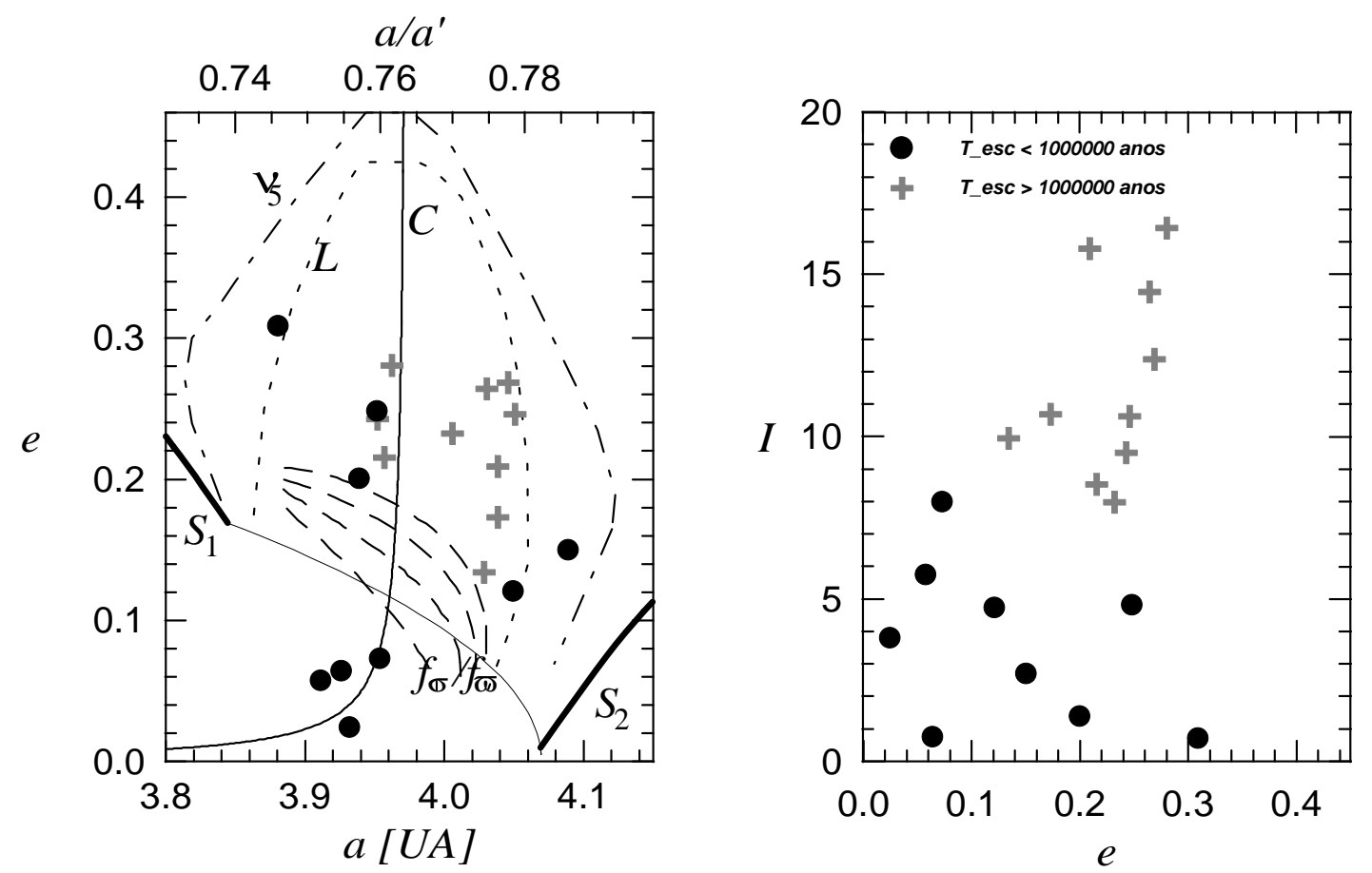

Figura 5.29: Posição inicial dos objetos que escaparam durante a integração, no caso em que consideramos $5 n_{\mathrm{S}}-2 n_{\mathrm{J}} \simeq 4704^{\prime \prime}$ /ano (aproximadamente 273 anos de período).

que acontece é que seja difícil conseguir um batimento exato entre as freqüências devido a que o espectro de freqüências de $\sigma$ apresenta poucas linhas e todas muito isoladas (ver Apêndice A, Seção A.2). A única forma de verificar esta conjetura com os nossos resultados, seria fazer uma análise de Fourier das soluções fornecidas pelo mapeamento. Isto seria perfeitamente possível com os dados de que dispomos, mas, a implementação desta análise fica além dos objetivos propostos para esta tese.

Para mostrar o comportamento dos objetos nesta ressonância apresentamos alguns exemplos nas Figs. 5.31 - 5.34. Todos os objetos que não escapam se comportam de forma muito regular, como a apresentada na Fig. 5.31. Novamente, o espalhamento que aparece nas Figs. 5.31a,c é causado por um efeito de projeção sobre a superfície de seção, e a Fig. 5.31d demonstra que o que observamos é, na verdade, uma libração de grande amplitude em $e$.

Além dos asteróides do grupo de Chicago, em quase todos os testes feitos aparecem alguns poucos objetos que escapam em tempos de $\sim 10^{7}$ anos. O principal exemplo é o asteróide 2760 Kacha, cujo comportamento e mostrado na Fig. 5.32. 


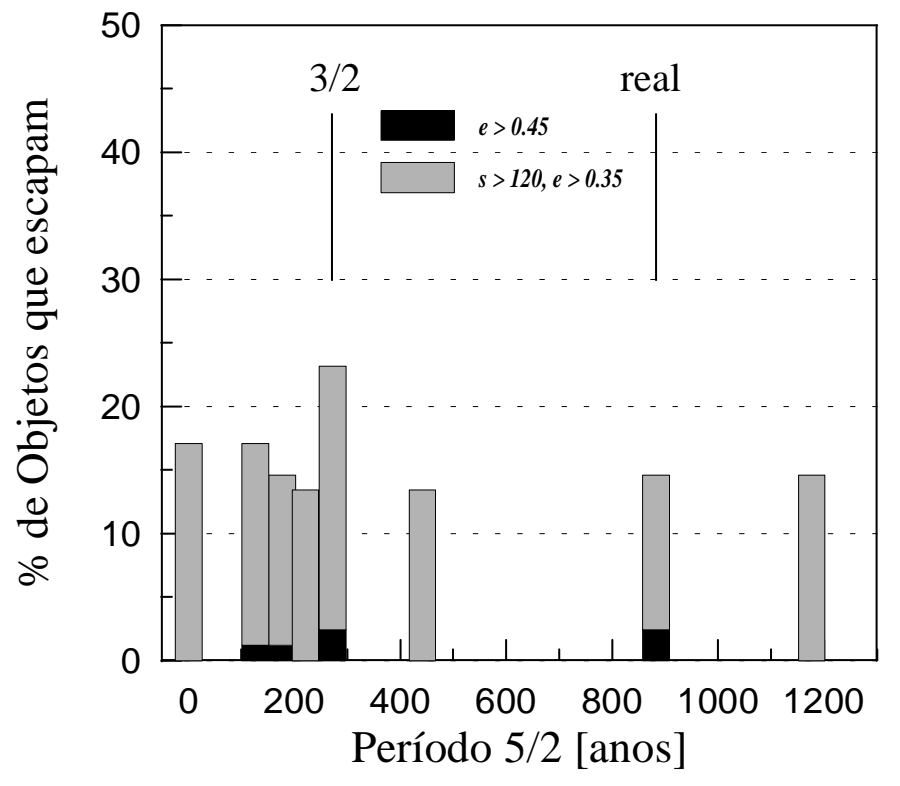

Figura 5.30: Ressonância 3/2. Porcentagem de objetos que escapam (sobre o total das 82 condições testadas) em $T<10^{8}$ anos, como função do valor do período $2 \pi /\left(5 n_{\mathrm{S}}-\right.$ $2 n_{\mathrm{J}}$ ) das perturbações devidas à comensurabilidade $5 / 2$ entre Júpiter e Saturno. Os tons preto e cinza permitem distinguir entre os diferentes tipos de escape. Aparecem indicados os valores do período real da comensurabilidade $5 / 2$ e do período de libração de $\sigma$ na ressonância $3 / 2$.

Os objetos mais interessantes são aqueles cujo comportamento é afetado pela mudança no valor de $5 n_{\mathrm{S}}-2 n_{\mathrm{J}}$. Um bom exemplo é o asteróide 361 Bononia, que mostra um comportamento muito regular quando consideramos o valor real de $5 n_{\mathrm{S}}-2 n_{\mathrm{J}}$ (Fig. 5.33), mas torna-se muito irregular quando o período desta freqüência é $\sim 273$ anos (Fig. 5.34).

No capítulo seguinte, discutiremos nossos resultados e analisaremos algumas perspectivas de pesquisas futuras sobre o tema. 
a.

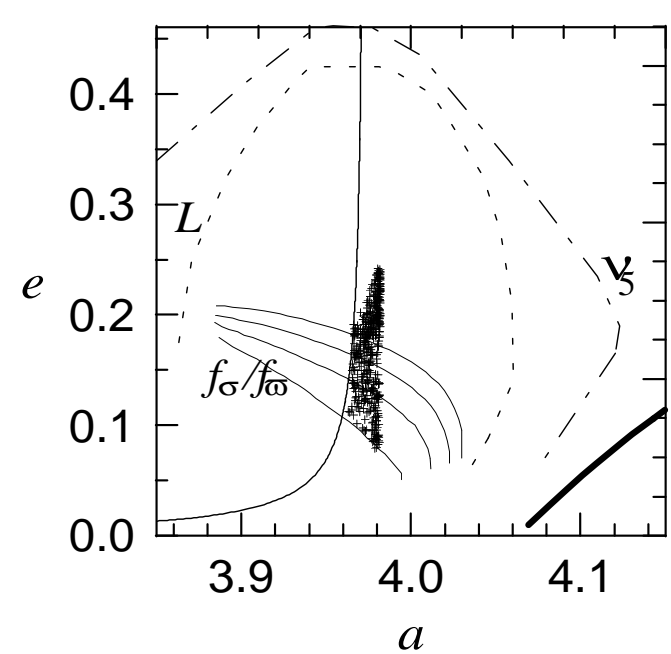

c.

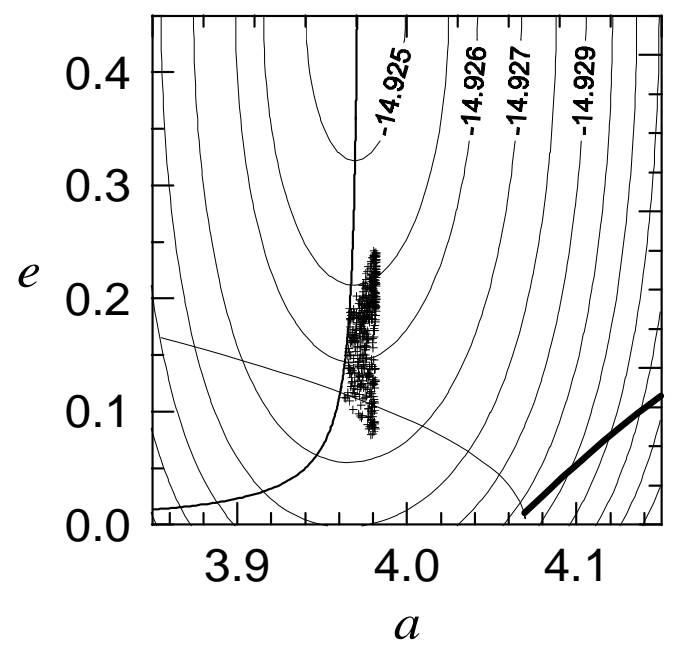

b.

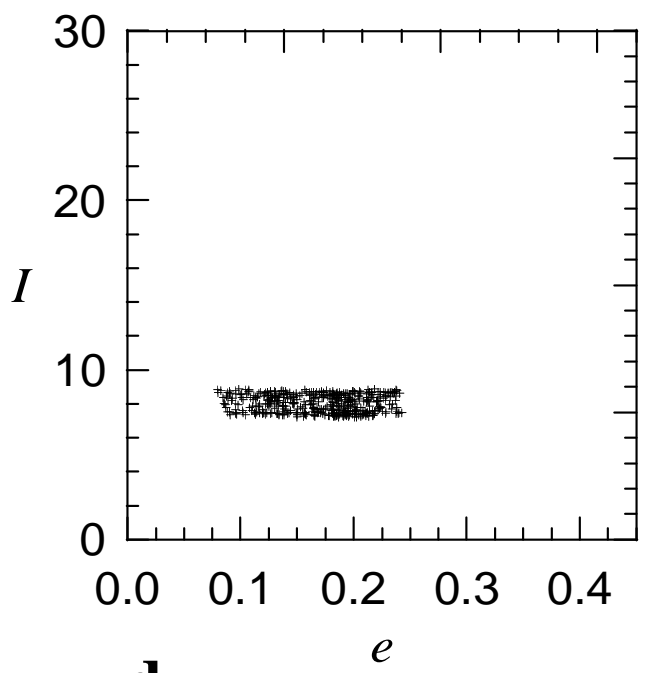

d.

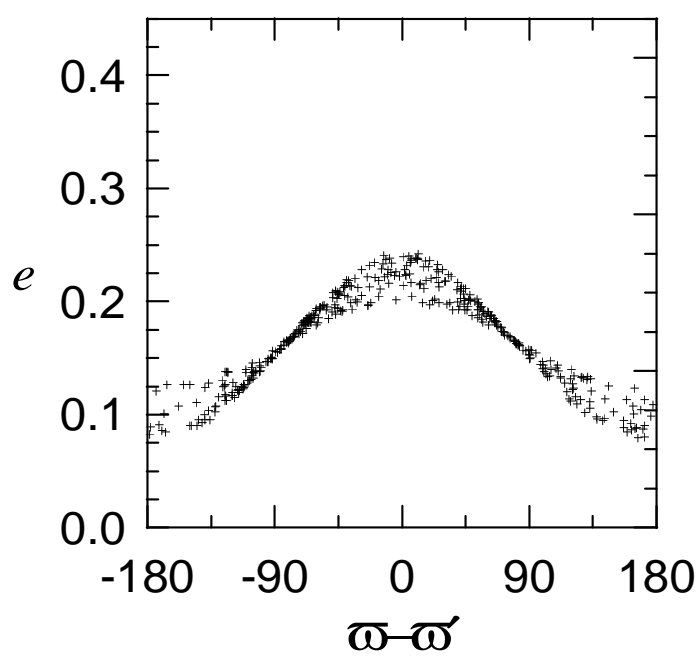

Figura 5.31: Comportamento do asteróide 153 Hilda, considerando o valor real da freqüência da comensurabilidade 5/2 entre Júpiter e Saturno. A superfície de seção é a mesma utilizada em exemplos anteriores. 
a.

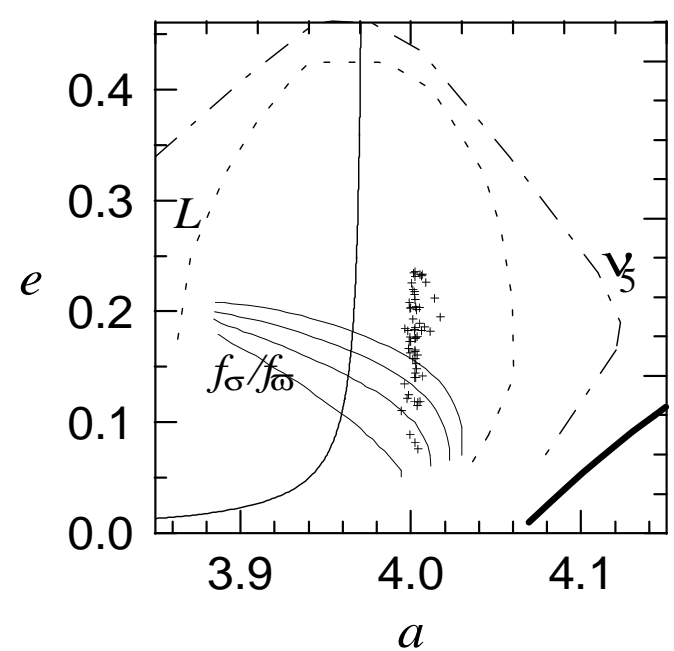

c.

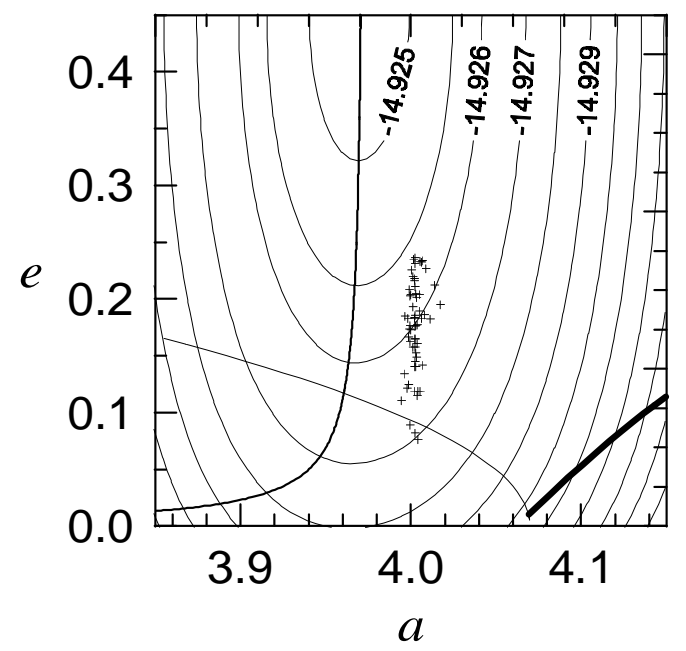

b.

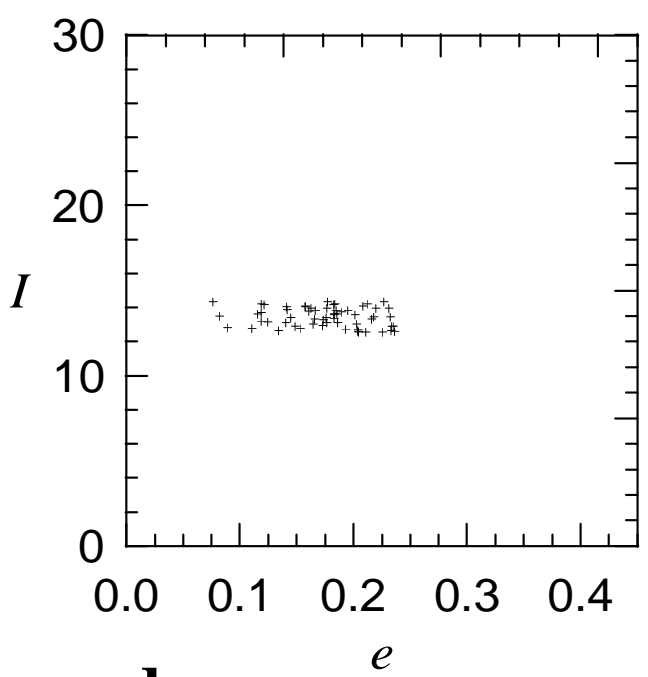

d.

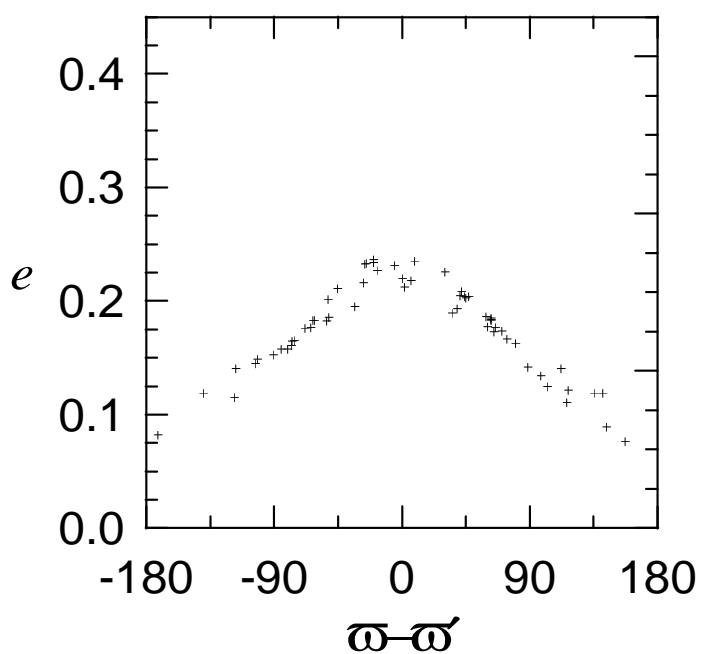

Figura 5.32: Comportamento do asteróide 2760 Kacha, considerando o valor real da freqüência da ressonância $5 / 2$ entre Júpiter e Saturno. Este objeto escapa quando $|\sigma|>120^{\circ}$ e $e>0.35$. 
a.

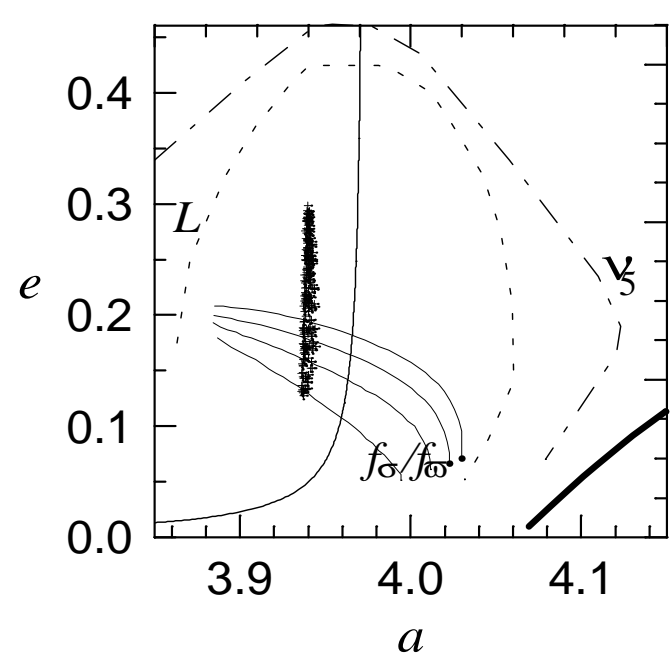

c.

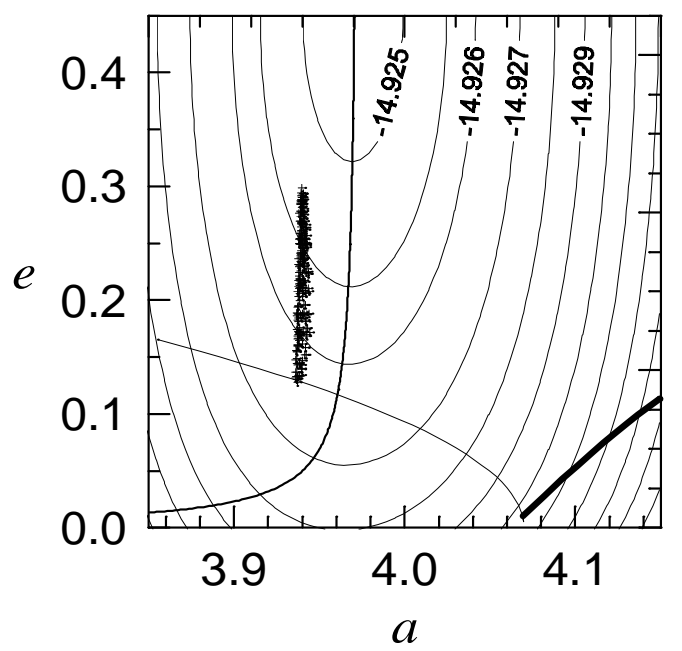

b.

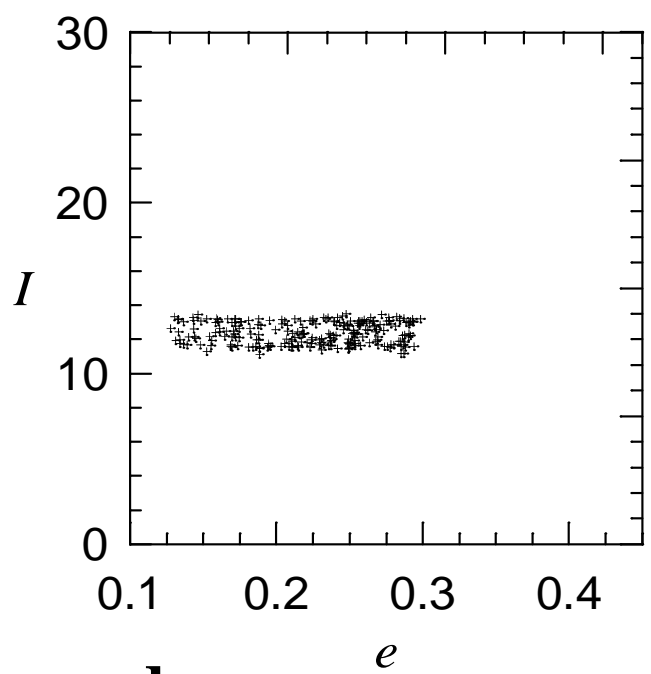

d.

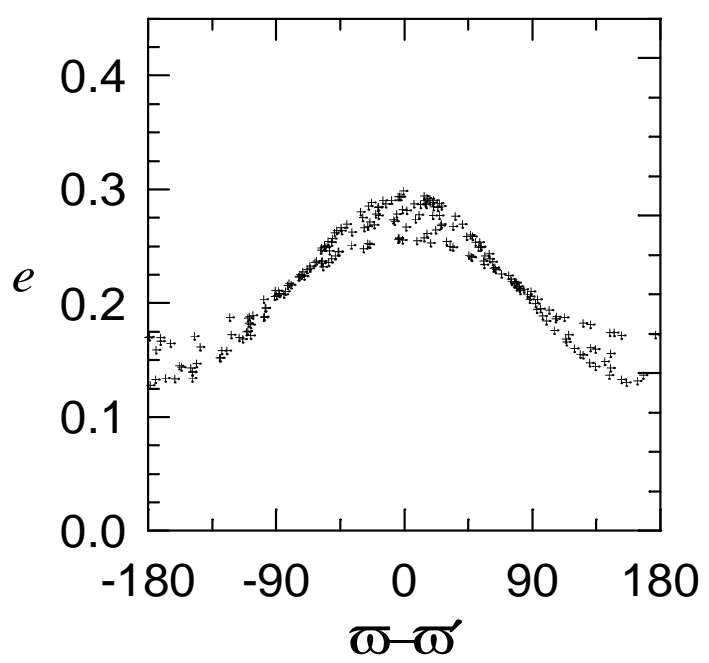

Figura 5.33: Comportamento do asteróide 361 Bononia, considerando o valor real da freqüência da comensurabilidade $5 / 2$ entre Júpiter e Saturno. 
a.

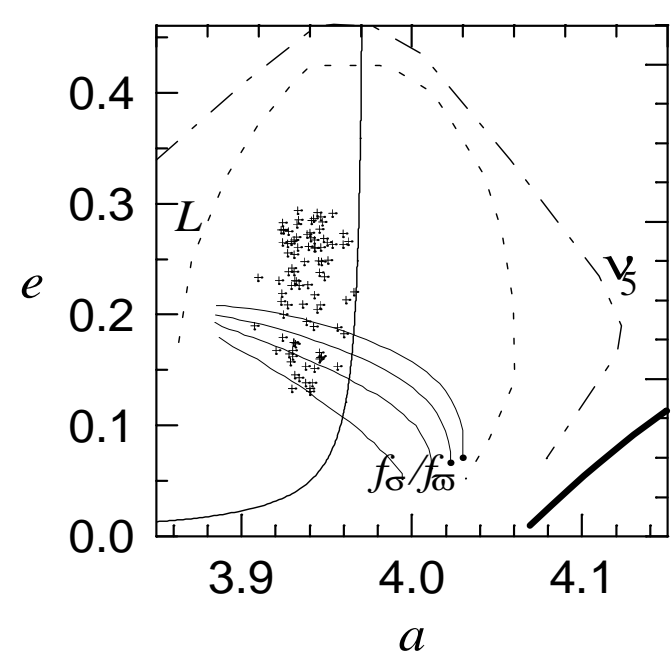

c.

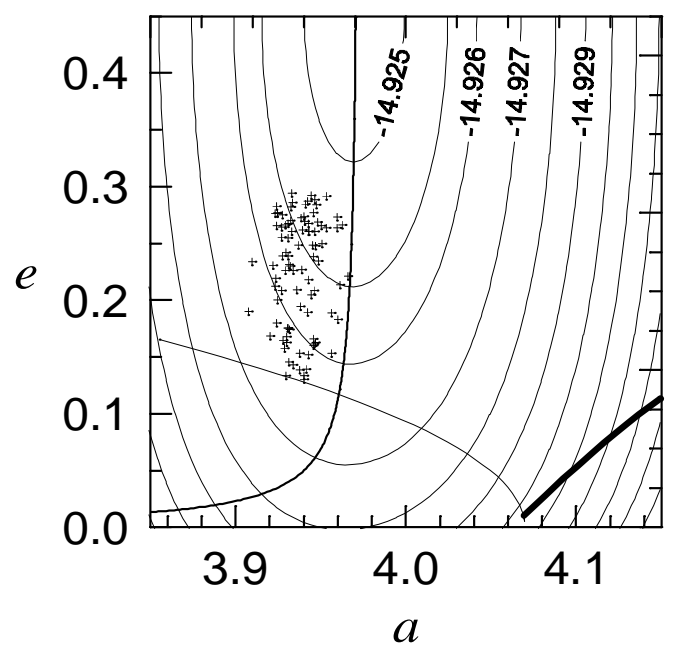

b.

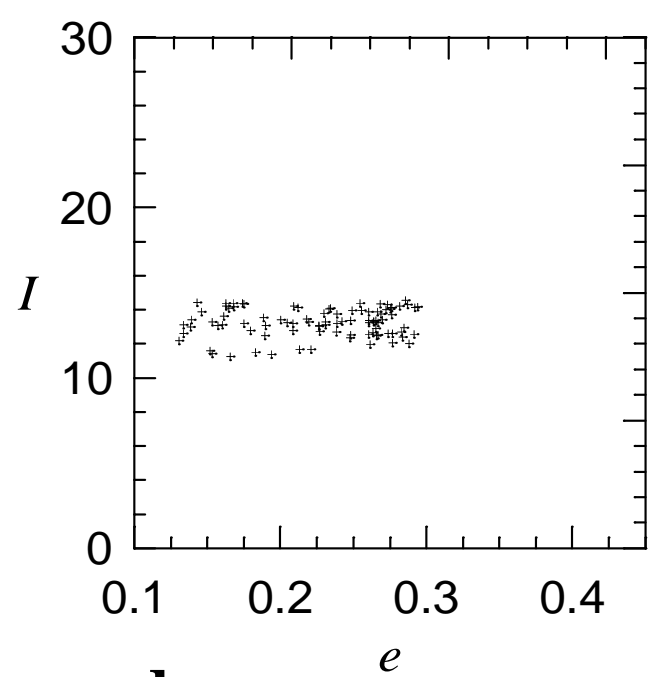

d.

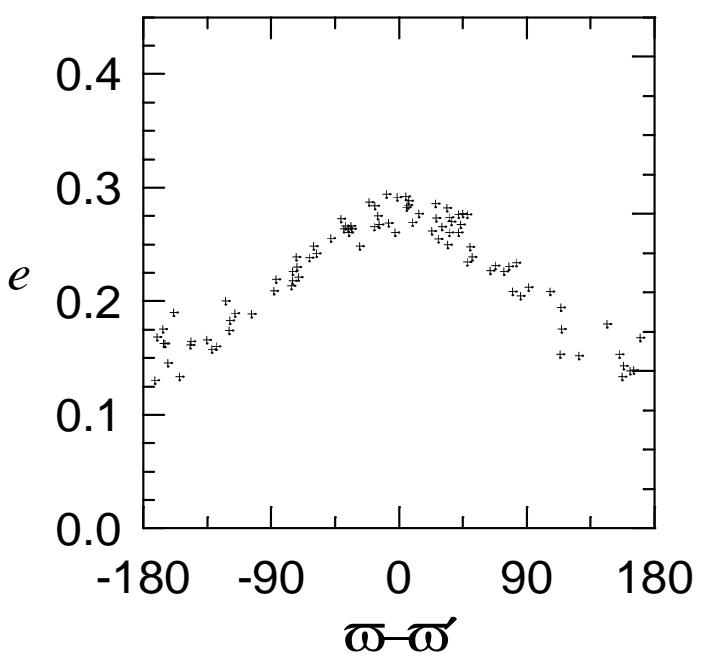

Figura 5.34: Comportamento do asteróide 361 Bononia, considerando a freqüência $5 n_{S}-2 n_{J} \simeq 4704^{\prime \prime} /$ ano (período de 273 anos). É notória a diferença com a Fig. 5.33. 


\section{Conclusões e Perspectivas futuras}

O objetivo principal desta tese foi estender os resultados do mapeamento simplético do modelo plano (Ferraz-Mello, 1997) para o caso espacial. Isto envolveu duas etapas principais: por um lado, a formulação de um desenvolvimento assimétrico da função perturbadora adequado para o problema que queríamos estudar; por outro lado, a montagem do mapeamento e a obtenção dos resultados. Podemos dizer que ambas etapas foram bem sucedidas, e que cumprimos satisfatoriamente com o objetivo proposto.

Não há dúvidas de que a combinação mapeamento simplético + desenvolvimento assimétrico, constitui um modelo ótimo para realizar estudos qualitativos de caráter exploratório nas ressonâncias asteroidais. Mesmo sendo um modelo muito aproximado, ele mostra seu poder ao conseguir reproduzir bem as principais características dinâmicas conhecidas das diferentes regiões das ressonâncias $2 / 1$ e $3 / 2$. Isto, somado à velocidade de cálculo, torna-o uma ferramenta extremamente útil para analisar o comportamento ressonante em intervalos de tempo muito longos, da ordem da idade do Sistema Solar.

Esta última afirmação pode parecer contraditória se levamos em conta que o tempo de integração dos testes apresentados neste trabalho não foi além de $10^{8}$ anos, o que certamente não é uma escala cosmogônica. No entanto, o escasso intervalo de tempo analisado é compensado com o grande número de condições iniciais estudadas (por volta de 1300 condições na ressonância $2 / 1$ e 650 na ressonância 3/2), o que fornece aos resultados obtidos uma significância estatística importante.

Nossos resultados permitem confirmar, sem nenhum tipo de dúvidas, o fato de que 
as variações de curto período da órbita de Júpiter que ocorrem na comensurabilidade $5 / 2$ entre este planeta e Saturno (grande desigualdade), jogam um papel fundamental na depleção das ressonâncias $2 / 1$ e 3/2. Particularmente, quando o período destas variações é muito próximo do período de libração de $\sigma$, a difusão caótica é sensivelmente intensificada. No caso da ressonância $2 / 1$, isto contribui ao rápido esvaziamento da região com excentricidades moderadas $(0.2<e<0.4)$ em torno do ramo pericêntrico, e também da região com $0.3<e<0.4$ e amplitudes de libração moderadas $\left(50^{\circ}<\right.$ $\sigma<90^{\circ}$ ). No caso da ressonância $3 / 2$, o efeito das variações de curto período da grande desigualdade sobre o movimento dos Hildas não é tão evidente, mas ainda existe. Acreditamos que um estudo detalhado das freqüências próprias do movimento dos Hildas poderia ajudar a compreender melhor a forma em que a "grande desigualdade" atua neste caso.

No entanto, há ainda alguns interrogantes a serem respondidos, relacionados às regiões de aparente estabilidade observadas nestas duas ressonâncias. No caso da ressonância $2 / 1$, uma das regiões de interesse é aquela com $0.15<e<0.3$ e amplitudes de libração $60^{\circ}<\sigma<90^{\circ}$, onde localiza-se o grupo de Zhongguo. No caso da ressonância $3 / 2$, é a região em torno do ramo pericêntrico ocupada pela maioria dos Hildas. A dinâmica destas regiões não pode ser desvendada em intervalos de tempo curtos, e precisa-se de simulações por tempos da ordem da idade do Sistema Solar, acompanhadas de metodologias que permitam quantificar o grau de instabilidade destas regiões (indicadores de caoticidade confiáveis). Estas simulações podem ser realizadas utilizando o mapeamento simplético apresentado neste trabalho, como também utilizando modelos mais precisos, tanto numéricos quanto analíticos, e abrem todo um vasto panorama para pesquisas futuras. 


\section{BIBLIOGRAFIA}

Beaugé C., 1994, "Asymmetric librations in exterior resonances", Cel. Mech. Dyn. Astr. 60, 225-248

Beaugé C., 1996, "On a global expansion of the disturbing function in the planar elliptic restricted three-body problem", Cel. Mech. Dyn. Astr. 64, 313-350

Bowell E., Muinonen K. e Wasserman L., 1994, "A public-domain asteroid orbit database", em Asteroids, Comets and Meteors (IAU Symp. 160), A. Milani et al. (eds.), Kluwer Academic Publishers, pp. 477-481

Candy J. e Rozmus W., 1991, "A symplectic integration algorithm for separable Hamiltonian functions", J. Comp. Phys. 92, 230-256

Carpino M., Milani A. e Nobili A.M., 1987, "Long-term numerical integrations and synthetic theories for the motion of the outer planets", Astron. Astrophys. 181, 182-194

Channell P.J. e Scovel C., 1990, "Symplectic integration for Hamiltonian systems", Nonlinearity 3, 231-259

Chirikov B.V., 1979, "A universal instability of many-dimensional oscillator systems", Physics Reports 52, 264-379

Cordeiro R.R., Gomes R.S. e Martins R.V., 1996, "A mapping for non-conservative systems", preprint

Danby J.M.A., 1988, "Fundamentals of Celestial Mechanics", Willmann-Bell

Duncan M., Quinn T. e Tremaine S., 1989, "The long-term dynamical evolution of 
orbits in the Solar System: a mapping approach", Icarus 82, 402-418

Everhart E., 1985, "An efficient integrator that uses Gauss-Radau spacings", em IAU Colloquium 83, A. Carusi e G. Valsecchi (eds.), Reidel Pub., pp. 185-202

Ferraz-Mello S., 1987, "Expansion of the disturbing force-function for the study of high-eccentricity librations", Astron. Astrophys. 183, 397-402

Ferraz-Mello S., 1988, "The high-eccentricity libration of the Hildas", Astron. J. 96, $400-408$

Ferraz-Mello S., 1989, "A semi-numerical expansion of the averaged disturbing function for some very-high-eccentricity orbits", Celest. Mech. 45, 65-68

Ferraz-Mello S., 1994a, "The convergence domain of the Laplacian expansion of the disturbing function", Cel. Mech. Dyn. Astr. 58, 37-52

Ferraz-Mello S., 1994b, "Dynamics of the $2 / 1$ asteroidal resonance", Astron. J. 108, $2330-2337$

Ferraz-Mello S., 1997, "A symplectic mapping approach to the study of the stochasticity of asteroidal resonances", Cel. Mech, Dyn. Astr. 65, 421-437

Ferraz-Mello S. e Klafke J.C., 1991, "A model for the study of very-high-eccentricity asteroidal motion. The 3/1 resonance", em Predictability, Stability and Chaos in $N$-body Dynamical Systems, A.E. Roy (ed.), Plenum Press, pp. 177-184

Ferraz-Mello S. e Michtchenko T., 1996, "Orbital evolution of asteroids in the Hecuba gap", em The Dynamical Behaviour of our Planetary System, J. Henrard et al. (eds.), Kluwer Academic Publishers

Ferraz-Mello S. e Roig F., 1996, "Comparative study of symplectic mappings in resonant systems", Rev. Mex. Astron. Astrofis., Serie de conferencias, vol. 4 (resumo)

Ferraz-Mello S. e Sato M., 1989, "The very-high-eccentricity asymmetric expansion of the disturbing function near resonances of any order", Astron. Astrophys. 225, $541-547$

Forest E. e Ruth R.D., 1990, "Fourth-order symplectic integration", Physica D 43, $105-117$

Forest E., Bengtsson J. e Reusch M., 1991, "Application of the Yoshida-Ruth techniques to implicit integration and multi-map explicit integration", Phys. Lett. A 158, 99101

Froeschlé Cl., 1992, "Mappings in astrodynamics", em Chaos, Resonance and Collective 
Dynamical Phenomena in the Solar System, S. Ferraz-Mello (ed.), Kluwer Academic Publishers, pp. 375-390

Froeschlé Cl. e Petit J.M., 1990, "Polynomial approximation of Poincaré maps for Hamiltonian systems", Astron. Astrophys. 238, 413-423

Giffen R., 1973, "A study of commensurable motion in the asteroid belt", Astron. Astrophys. 23, 387-403

Gladman B., Duncan M. e Candy J., 1991, "Symplectic integrators for long-term integrations in celestial mechanics", Cel. Mech. Dyn. Astr. 52, 221-240

Hadjidemetriou J.D., 1986, "A hyperbolic twist mapping model for the study of asteroid orbits near the $3 / 1$ resonance", J. Appl. Math. Phys. 37, 776-796

Hadjidemetriou J.D., 1988, "Algebraic mappings near a resonance with an application to asteroid motion", em Long Term Dynamical Behaviour of Natural and Artificial N-body Systems, A.E. Roy (ed.), Kluwer Academic Publishers, pp. 257-276

Hadjidemetriou J.D., 1991, "Mapping models for Hamiltonian systems with application to ressonant asteroidal motion", em Predictability, Stability and Chaos in N-body Dynamical Systems, A.E. Roy (ed.), Plenum Press, pp. 157-175

Hadjidemetriou J.D., 1993, "Asteroid motion near the 3/1 resonance", Cel. Mech. Dyn. Astr. 56, 563-599

Hadjidemetriou J.D., 1996, "Symplectic mappings", preprint

Henrard J., 1997, "Symplectic integrators", em Analysis and Modelling the Discrete Dynamical Systems, with Application to Dynamical Astronomy, D. Benest e C. Froeschlé (eds.), Gordon \& Breach

Henrard J. e Lemaitre A., 1983, " A second fundamental model for resonance", Celest. Mech. 30, 197-281

Henrard J. e Lemaitre A., 1986, "A perturbation method for problems with two critical arguments", Celest. Mech. 39, 213-238

Henrard J., Watanabe N. e Moons M., 1995, “A bridge between secondary and secular resonances inside the Hecuba gap", Icarus 115, 336-346

Kinoshita H. e Nakai H., 1992, "New methods for long-time numerical integration of planetary orbits", em Chaos, Resonance and Collective Dynamical Phenomena in the Solar System, S. Ferraz-Mello (ed.), Kluwer Academic Publishers, pp. 395-406

Kinoshita H., Yoshida H. e Nakai H., 1991, "Symplectic integrators and their applica- 
tion to dynamical astronomy", Cel. Mech. Dyn. Astr. 50, 59-71

Klafke J.C., Ferraz-Mello S. e Michtchenko T., 1992, "Very-high-eccentricity librations at some higher order resonance", em Chaos, Resonance and Collective Dynamical Phenomena in the Solar System, S. Ferraz-Mello (ed.), Kluwer Academic Publishers, pp. $153-158$

Lanczos C., 1974, "The Variational Principles of Mechanics", University of Toronto Press

Laskar J., Froeschlé Cl. e Celletti A., 1992, "The mesure of chaos by the numerical analysis of the fundamental frequencies. Application to the standard mapping", Physica D 56, 253-269

Lemaitre A. e Henrard J., 1990, "On the origin of chaotic behaviour in the 2/1 Kirkwood gap", Icarus 83, 391-409

Levinson H. e Duncan M., 1994, "The long-term dynamical behaviour of short-period comets", Icarus 108, 18-36

Malhotra R., 1994, "A mapping method for the gravitational few-body problem with dissipation", Cel. Mech. Dyn. Astr. 60, 373-385

Mersman W., 1971, "Explicit recursive algorithms for the construction of equivalent canonical transformations", Celest. Mech. 3, 384-389

Michtchenko T. e Ferraz-Mello S., 1995, "Comparative study of the asteroidal motion in the $3 / 2$ and $2 / 1$ resonances with Jupiter I. Planar model", Astron. Astrophys. 303, $945-963$

Michtchenko T. e Ferraz-Mello S., 1996, "Comparative study of the asteroidal motion in the $3 / 2$ and $2 / 1$ resonances with Jupiter II. Three-dimensional model", Astron. Astrophys. 310, 1021-1035

Michtchenko T. e Ferraz-Mello S., 1997, "Escape of asteroids from the Hecuba gap", em publicação em Planetary and Space Science

Milani A., Nobili A.M. e Carpino M., 1987, "Secular variations of semimajor axes: theory and experiments", Astron. Astrphys. 172, 265-279

Moons M., 1993, "On the resonant Hamiltonian in the restricted three-body problem", Dept. of Math. Report 19, FUNDP, Namur

Moons M. e Morbidelli A., 1995, "Secular resonances in mean motion commensurabilities: the $4 / 1,3 / 1,5 / 2$ and $7 / 3$ cases", Icarus $114,33-50$ 
Morbidelli A., 1996, "The Kirkwood gap at the 2/1 commensurability with Jupiter: new numerical results", Astron. J. 111, 2453-2461

Morbidelli A. e Moons M., 1993, "Secular resonances in mean motion commensurabilities: the $2 / 1$ and $3 / 2$ cases", Icarus $102,316-332$

Morbidelli A., Zappalà V., Moons M., Cellino A. e Gonczi R., 1995, "Asteroid families close to mean motion resonances: dynamical effects and physical implications", Icarus 118, 132-154

Murray C.D., 1986, "Structure of the 2/1 and 3/2 Jovian resonances", Icarus 65, 70-82

Nesvorný D. e Ferraz-Mello S., 1997a, "Chaotic diffusion in the 2/1 asteroidal resonance. An application of the frequency map analysis", Astron. Astrophys. 320, $672-680$

Nesvorný D. e Ferraz-Mello S., 1997b, "On the asteroidal population of the first-order Jovian resonances", em publicação em Icarus

Nobili A.M., Milani A. e Carpino M., 1989, "Fundamental frequencies and small divisors in the orbits of the outer planets", Astron. Astrophys. 210, 313-336

Petrosky T.V. e Broucke R., 1988, "Area-preserving mappings and deterministic chaos for nearly parabolic motions", Celest. Mech. 42, 53-79

Roig F., Simula A., Ferraz-Mello S. e Tsuchida M., 1997, "The high-eccentricity asymmetric expansion of the disturbing function for non-planar resonant problems", em publicação em Astron. Astrophys. (cf. Anexo I)

Ruth R.D., 1983, "A canonical integration technique", IEEE Trans. Nucl. Sci. NS-30, $2669-2671$

Saha P. e Tremaine S., 1992, "Symplectic integrators for Solar System dynamics", Astron. J. 104, 1633-1640

Šidlichovský M., 1992, "Mapping for the asteroidal resonances", Astron. Astrophys. 259, 341-348

Šidlichovský M., 1993, "Chaotic behaviour of trajectories for the asteroidal resonances", Cel. Mech. Dyn. Astr. 56, 143-152

Šidlichovský M., 1996, "Mapping and dynamical systems", em The Dynamical Behaviour of our Planetary System, J. Henrard et al. (eds.), Kluwer Academic Publishers

Šidlichovský M. e Melendo B., 1986, "Mapping for 5/2 asteroidal commensurability", 
Bull. Astron. Inst. Czechoslov. 37, 65-80

Šidlichovský M. e Nesvorný D., 1997, "Frequency modified Fourier transform and its application to asteroids", Cel. Mech. Dyn. Astr. 65, 137-148

Simon J.L. e Bretagnon P., 1975, "Résultats des perturbations du premier ordre des quatre grosses planètes. Variations littérales", Astron. Astrphys. Suppl. Ser. 22, $107-160$

Simon J.L. e Bretagnon P., 1984, "Théorie du mouvement de Jupiter et Saturne sur un intervalle de temps de 6000 ans. Solution JASON84", Astron. Astrophys. 138, $169-178$

Varadarajan V.S., 1974, "Lie groups, Lie algebras and their representation", PrenticeHall

Whittaker E.T. e Watson G.N., 1950, “A Course on Modern Analysis", Cambridge University Press

Wintner A., 1941, "The Analytical Foundations of Celestial Mechanics", Princeton University Press

Wisdom J., 1980, "The resonance overlap criterion and the onset of stochastic behavior in the restricted three-body problem", Astron. J. 85, 1122-1133

Wisdom J., 1982, "The origin of the Kirkwood gaps: a mapping for asteroidal motion near the $3 / 1$ commensurability", Astron. J. 87, 577-593

Wisdom J., 1983, "Chaotic behavior and the origin of the 3/1 Kirkwood gap", Icarus 56, $51-74$

Wisdom J., 1987, "Urey Prize Lecture: Chaotic dynamics in the Solar System", Icarus 72, 241-275

Wisdom J. e Holman M., 1992a, "Symplectic maps for the $n$-body problem", Astron. J. 102, 1528-1538

Wisdom J. e Holman M., 1992b, "Symplectic maps for the $n$-body problem: stability analysis", Astron. J. 104, 2022-2029

Wisdom J., Holman M. e Touma J., 1994, "Symplectic correctors", preprint

Yokoyama T., 1994, "Expansion of the disturbing function for high-eccentricity and large amplitude of libration", Cel. Mech. Dyn. Astr. 60, 387-400

Yoshida H., 1990, "Construction of higher order symplectic integrators", Phys. Lett. A 150, 262-268 
Yoshida H., 1992, "Symplectic integrators for Hamiltonian systems: basic theory", em Chaos, Resonance and Collective Dynamical Phenomena in the Solar System, S. Ferraz-Mello (ed.), Kluwer Academic Publishers, pp. 407-411

Yoshida H., 1993, "Recent progress in the theory and application of symplectic integrators", Cel. Mech. Dyn. Astr. 56, 27-43

Yoshikawa M., 1990, "Motion of asteroids at the Kirkwood gaps I. On the 3/1 resonance with Jupiter", Icarus 87, 78-102

Yoshikawa M., 1991, "Motion of asteroids at the Kirkwood gaps II. On the 5/2, 7/3 and 2/1 resonances with Jupiter", Icarus 92, 94-117 


\section{APÊNDICE A}

\section{CONCEITOS BÁSICOS SOBRE RESSONÂNCIAS} ASTEROIDAIS

Apresentamos neste apêndice uma breve introdução ao estudo das ressonâncias de primeira ordem no cinturão de asteróides, pondo especial ênfase no que é conhecido atualmente sobre a dinâmica das ressonâncias $2 / 1$ e $3 / 2$. Este apêndice serve como complemento ao que já foi discutido ao longo da tese.

\section{A.1. A dinâmica do problema ressonante}

O fenômeno de ressonância está associado à libração de alguma das variáveis angulares que descreve a evolução dinâmica do sistema ressonante. Esta variável recebe o nome de argumento crítico da ressonância e, em geral, sua variação temporal é muito lenta em comparação com os restantes ângulos. Assim, é possível reduzir os graus de liberdade do problema através da aplicação adequada do método de média; a dinâmica da ressonância fica então descrita por uma Hamiltoniana média que descreve a evolução do sistema no longo prazo.

No caso particular do problema restrito dos três corpos, a existência de uma ressonância entre os movimentos médios do perturbado $(n)$ e do perturbador $\left(n^{\prime}\right)$ da forma

$$
\frac{n}{n^{\prime}} \simeq \frac{p+q}{p}
$$


( $p, q$ inteiros), traduz-se na libração do argumento crítico

$$
\sigma=\frac{p+q}{q} \lambda^{\prime}-\frac{p}{q} \lambda-\varpi
$$

( $\lambda$ e $\varpi$ são as longitudes média e do periélio, respectivamente) em torno de um certo valor $\sigma_{0}$ que depende da ordem da ressonância $q$. Quando consideramos o problema restrito circular $\left(e^{\prime}=0\right)$ e plano, a aplicação do citado método de média permite reduzir a Hamiltoniana à forma

$$
\langle\mathcal{H}(\sigma, J)\rangle=c_{1} J+\frac{c_{2}}{2} J^{2}+\varepsilon(2 J)^{q / 2} \cos q \sigma
$$

onde o símbolo $\langle\cdots\rangle$ representa a média, $\varepsilon$ é o pequeno parâmetro da perturbação (a massa do perturbador), $J$ é ação conjugada a $\sigma$, e $c_{1}$ e $c_{2}$ são constantes vinculadas à freqüência fundamental do problema não perturbado. Esta Hamiltoniana recebe o nome de Hamiltoniana de Andoyer ou Segundo Modelo Fundamental de Ressonância (Henrard e Lemaitre, 1983) ${ }^{1}$. O sistema possui só um grau de liberdade e, portanto, é integrável. Ainda mais, o problema circular possui uma integral do movimento:

$$
P=\sqrt{a}\left[(p+1) / p-\sqrt{1-e^{2}}\right]
$$

( $a, e$ são semieixo e excentricidade).

Os pontos de equilíbrio da Hamiltoniana de Andoyer são dados pelas equações

$$
\frac{d J}{d t}=0, \quad \frac{d \sigma}{d t}=0
$$

enquanto que a estabilidade destes pontos fica definida pelo Hessiano de $\mathcal{H}$ calculado nos pontos de equilíbrio. Se hess $(\mathcal{H})>0$, o ponto de equilíbrio é estável, enquanto que se $\operatorname{hess}(\mathcal{H})<0$, ele é instável. No caso não perturbado $(\varepsilon=0)$, as soluções da Hamiltoniana de Andoyer, representadas em coordenadas polares $\sqrt{2 J} \cos \sigma, \sqrt{2 J} \sin \sigma$, são círculos concêntricos em torno da origem com raio $J=$ cte, $J>0$, e freqüências constantes

$$
\nu=c_{1}+c_{2} J
$$

Se $c_{1}$ e $c_{2}$ possuem o mesmo sinal, as trajetórias têm o mesmo sentido de rotação. No entanto, se $c_{1}$ e $c_{2}$ têm sinais diferentes, então existe um certo valor $J=-c_{1} / c_{2}$ tal

\footnotetext{
${ }^{1} \mathrm{~A}$ representação de algumas ressonâncias externas $(p<0)$ precisa levar em conta um termo a mais nesta Hamiltoniana (Beaugé, 1994).
} 
que a freqüência é nula e o ângulo $\sigma$ torna-se ressonante. Ao passar dos valores de $J<-c_{1} / c_{2}$ aos valores de $J>-c_{1} / c_{2}$ temos uma inversão no sentido de rotação das soluções.

Quando perturbamos o problema, a topologia das soluções muda. Se $c_{1}$ e $c_{2}$ têm o mesmo sinal não pode existir ressonância e as soluções continuam sendo círculos, mas agora deslocados com relação à origem. Se o sinal de $c_{1}$ e $c_{2}$ é diferente, então pode existir ressonância no caso não perturbado. No caso de uma ressonância de primeira ordem $(q=1)$, podemos substituir $c_{1}=-c_{2} J$ na Hamiltoniana (A.3); logo obtemos

$$
\mathcal{H}=-\frac{c_{2}}{2} J^{2}+\varepsilon \sqrt{2 J} \cos \sigma
$$

ou seja, a Hamiltoniana de Andoyer se reduz àquela de um pêndulo. O sistema possui um ponto de equilíbrio estável em $\sigma_{0}=0$ e instável em $\sigma_{0}=\pi$. Para um valor fixo da integral $P$, as soluções para diferentes condições iniciais podem ser representadas no espaço de variáveis

$$
\begin{aligned}
& k=e \cos \sigma \\
& h=e \sin \sigma
\end{aligned}
$$

como na Fig. A.1a. Nessa figura, a região L corresponde à região das librações, a região $\mathrm{C}$ às circulações externas ou pericêntricas, e a região $\mathrm{A}$ às circulações internas ou apocêntricas. A separação entre estas três regiões é definida pela separatriz $\mathrm{S}_{\mathrm{x}}{ }^{2}$

Alternativamente, o movimento pode ser estudado no espaço $a, e$ analisando-se a projeção do eixo $k$ nesse espaço como na Fig. A.1b. Esta projeção constitui uma curva $P=$ cte. ao longo da qual acontece o movimento. O conjunto de pontos de equilíbrio estável para diferentes valores de $P$ forma uma curva contínua $C$ no espaço $a, e$ que recebe o nome de ramo pericêntrico e que é definida pela lei de estrutura da ressonância; a região de libração fica limitada pelos dois ramos da separatriz $S_{1}$ e $S_{2}$. Para valores de $P$ menores que um certo $P_{\text {lim }}$ a região de libração desaparece transformada numa região só de circulação em torno do ramo pericêntrico, que pode ser visualizada na Fig. A.1b para as excentricidades mais baixas onde não tem separatrizes. As projeções no espaço $a, e$ permitem visualizar a dinâmica do problema ressonante plano e os fenômenos difu-

\footnotetext{
${ }^{2}$ Perto da separatriz podem acontecer librações de amplitude muito grande que incluem a origem e, portanto, são semelhantes às circulações. Estas librações são as vezes chamadas de paradoxais.
} 


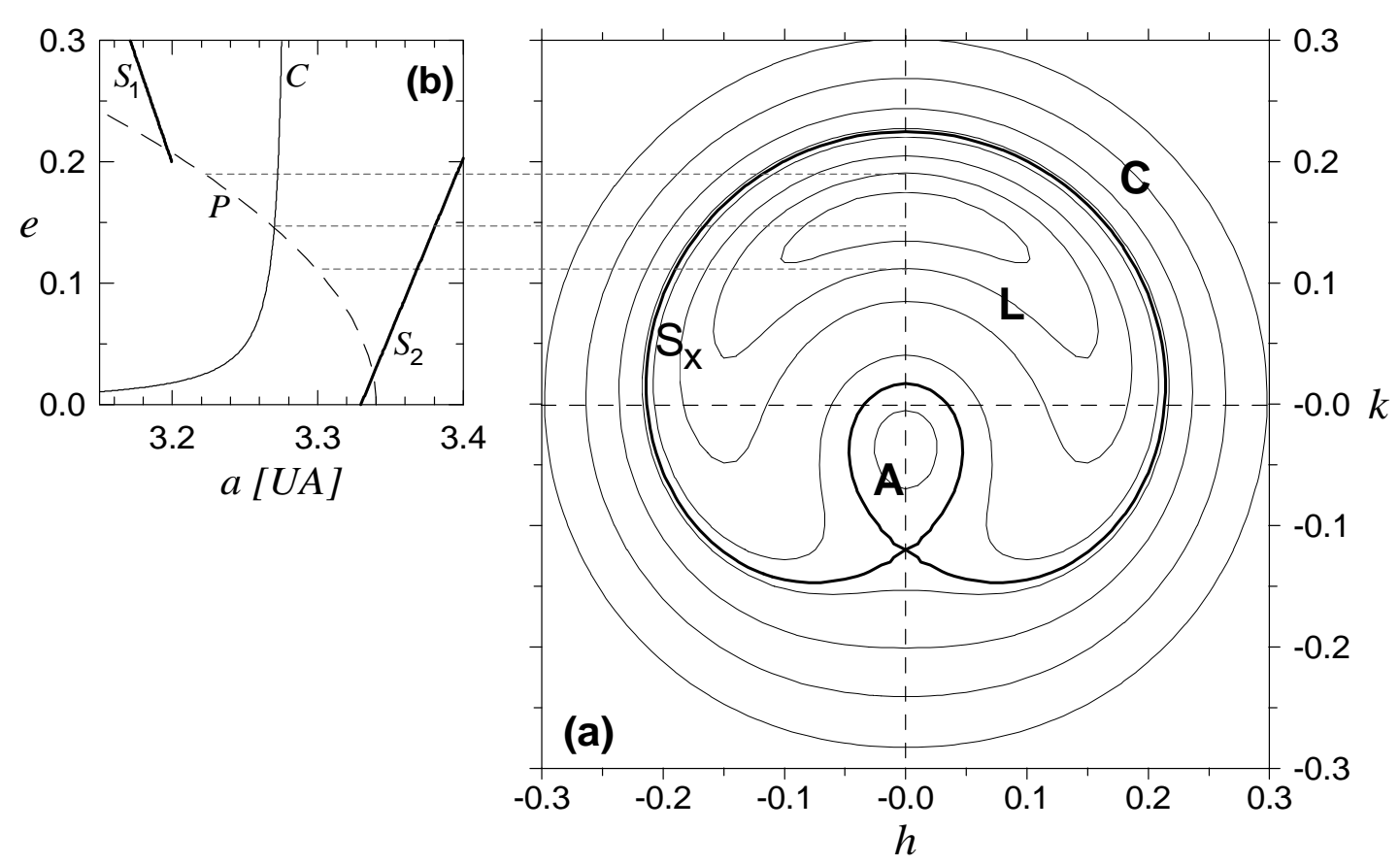

Figura A.1: A topologia do espaço de fase para uma ressonância de primeira ordem. (a) Soluções no espaço $k, h$ para $P=$ cte. (b) Projeção no espaço $a, e$.

sivos numa forma simples e bem caracterizada e, de forma análoga, existem projeções adequadas no espaço $e, I$ que permitem caracterizar a dinâmica do problema espacial.

Quando consideramos o problema elíptico, $P$ deixa de ser uma integral do movimento mas a topologia básica do espaço de fases é mantida. O movimento não acontece mais ao longo de uma curva $P=$ cte, mas ainda existe uma região de libração (L) em torno do ramo pericêntrico. A região de circulação por fora dos ramos da separatriz (C), e a região de circulação para excentricidades baixas (A), também são mantidas (teorema KAM). Porém, as soluções que se acham perto da separatriz podem difundir através desta, fazendo com que o sistema mude de regime, passando de libração para circulação, ou vice-versa, e gerando assim uma faixa estreita de movimentos caóticos. Quando o sistema possui vários graus de liberdade, mecanismos de difusão similares a este podem constituir vias de escape para que objetos que se acham originariamente numa certa região do espaço de fase possam migrar para outras regiões com condições dinâmicas diferentes: librações de alta excentricidade, "overlap" de ressonâncias seculares, etc. Esta migração poderia facilitar o escape de objetos da ressonância por colisões com os planetas interiores, e foi precisamente isto o que Wisdom (1982) e Ferraz-Mello 
e Klafke (1991) descobriram que acontecia na ressonância 3/1.

\section{A.2. As ressonâncias $2 / 1$ e $3 / 2$}

Estas duas ressonâncias, junto com a 4/3, são as ressonâncias de primeira ordem mais importantes no cinturão de asteróides. Por serem de primeira ordem, a sua topologia no espaço de fases é basicamente a mesma; no entanto elas estão associadas a fenômenos observacionais totalmente diferentes. A Fig. A.2 mostra a distribuição dos asteróides entre 2.5 e 5.2 UA junto com a localização das principais ressonâncias de movimentos médios. Podemos ver que a ressonância $2 / 1$ está associada a um vazio ou falha na distribuição: a falha de Hecuba; enquanto que a $3 / 2$ está associada a um grupo de asteróides: o grupo de Hilda. A ressonância 2/1 aparece limitada a direita por um grupo muito numeroso de objetos conhecido com família de Themis e a esquerda por um grupo menor cujo principal representante é o asteróide 65 Cybelle. Por sua vez, o grupo de Hilda está notoriamente isolado. Os avanços nas técnicas observacionais permitiram descobrir, recentemente, que a ressonância $2 / 1$ não está totalmente vazia e que existem uns 10 objetos com observações multi-oposicionais dentro da falha. No entanto, as novas descobertas levaram também a aumentar o número de objetos na ressonância $3 / 2$ de pouco menos de 60 a mais de 80 . A pergunta óbvia é: por que a ressonância 2/1 está praticamente despovoada em comparação com a ressonância $3 / 2$, sendo que as duas são de primeira ordem?

Em termos gerais, para $a<3.8$ UA as ressonâncias de baixa ordem estão vinculadas a falhas na distribuição (por exemplo, a $5 / 3$, a $7 / 3$, a $5 / 2$ ou a $3 / 1$ que não é mostrada na Fig. 2), e para $a>3.8 \mathrm{UA}$, as ressonâncias estão associadas a grupos (a $4 / 3$ possui o asteróide Thule, a $1 / 1$ os Trojanos). Como já mencionamos, após o trabalho pioneiro de Wisdom (1982), sabe-se que as falhas das ressonâncias 3/1, 5/2 e outras de alta ordem para $a<3.2$ UA podem ser conseqüência da difusão caótica das órbitas (através das separatrizes do problema circular) no espaço de fase (Šidlichovský e Melendo, 1986; Yoshikawa, 1990, 1991; Ferraz-Mello e Klafke, 1991; Moons e Morbidelli, 1995). Esta difusão seria causada pela superposição dos modos de oscilação forçados pela excentricidade de Júpiter, e induziria uma série de pulos na excentricidade do asteróide, levando-o a possíveis colisões com os planetas internos. Por sua vez, a notória ausência de asteróides para $a>4.2$ UA pode ser explicada através do "overlap" (critério de 


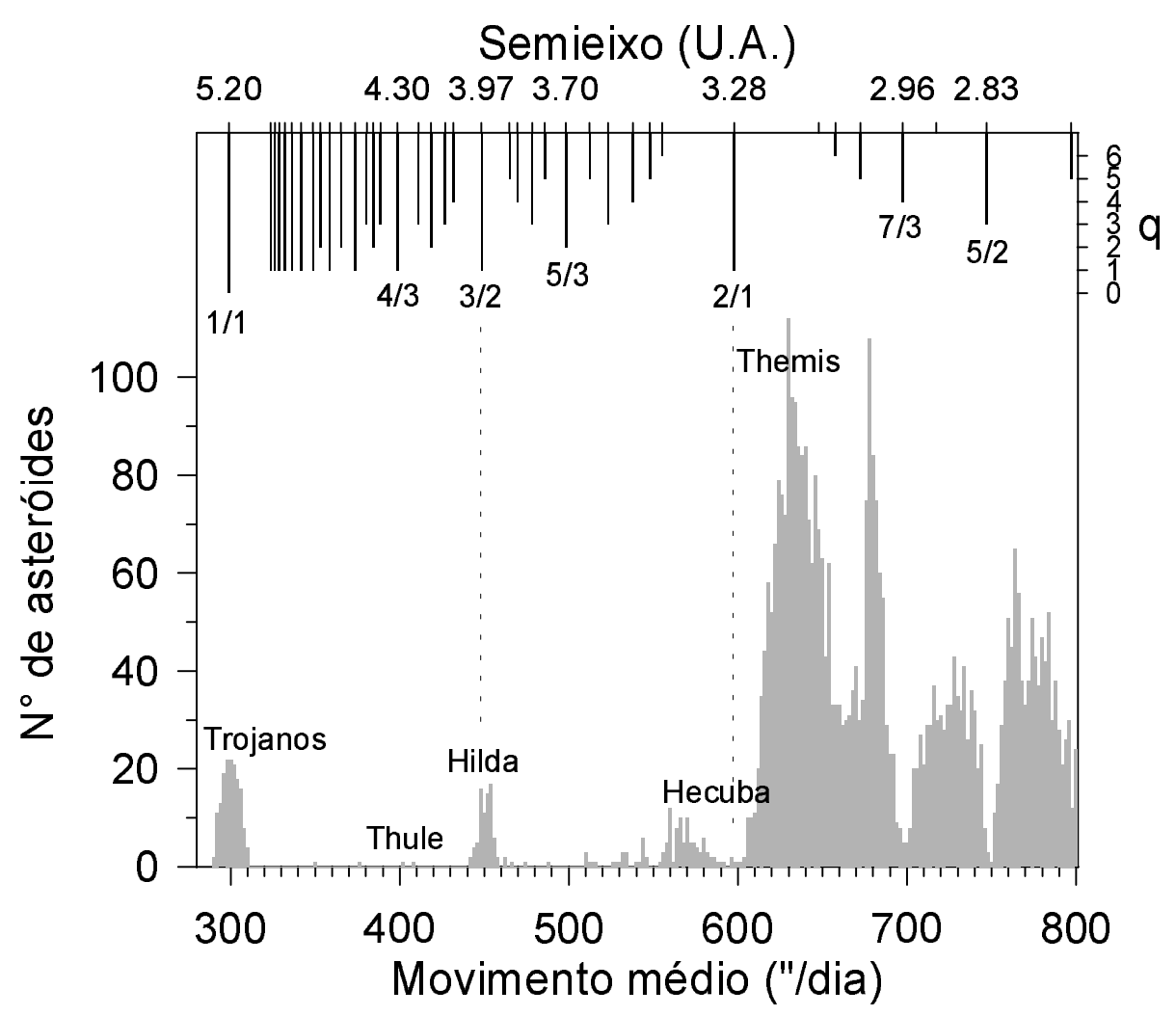

Figura A.2: Embaixo: distribuição dos asteróides entre 2.5 e 5.2 U.A. Acima: distribuição das ressonâncias de ordem $\leq 6$ no mesmo intervalo.

Chirikov) de ressonâncias de primeira ordem (Wisdom, 1980). O grupo dos Trojanos na ressonância $1 / 1$ seria mantido pela estabilidade dos pontos Lagrangeanos de Júpiter.

No entanto, entre 3.2 UA $<a<4.2$ UA o mecanismo dinâmico de depleção parece ser muito complexo e a questão ainda não está resolvida. Sabe-se que as ressonâncias $2 / 1$ e $3 / 2$ possuem uma estrutura dinâmica muito complexa, estreitamente vinculada à teia de ressonâncias seculares e secundárias na região. Aparentemente, o comportamento dos objetos dentro destas ressonâncias não pode ser explicado através de um único mecanismo dinâmico.

\section{A.2.1. A ressonância $2 / 1$}

A Fig. A.3 apresenta o ramo pericêntrico $(C)$ de soluções estacionárias estáveis, e as separatrizes $\left(S_{1}, S_{2}\right)$ da ressonância $2 / 1$, conjuntamente com a posição de algumas das 
ressonâncias seculares $\left(\nu_{5}, \nu_{16}\right)$ e das principais ressonâncias secundárias $\left(f_{\sigma} / f_{\varpi}\right)^{3}$. A linha pontilhada indicada como $L$ é o limite inferior das separatrizes das ressonâncias seculares $\nu_{5}, \nu_{6}$ e da ressonância de Kozai $f_{\varpi} / f_{\Omega} \approx 1 / 1$. A linha contínua indicada como $P$ é o limite inferior da região de libração no problema restrito circular.

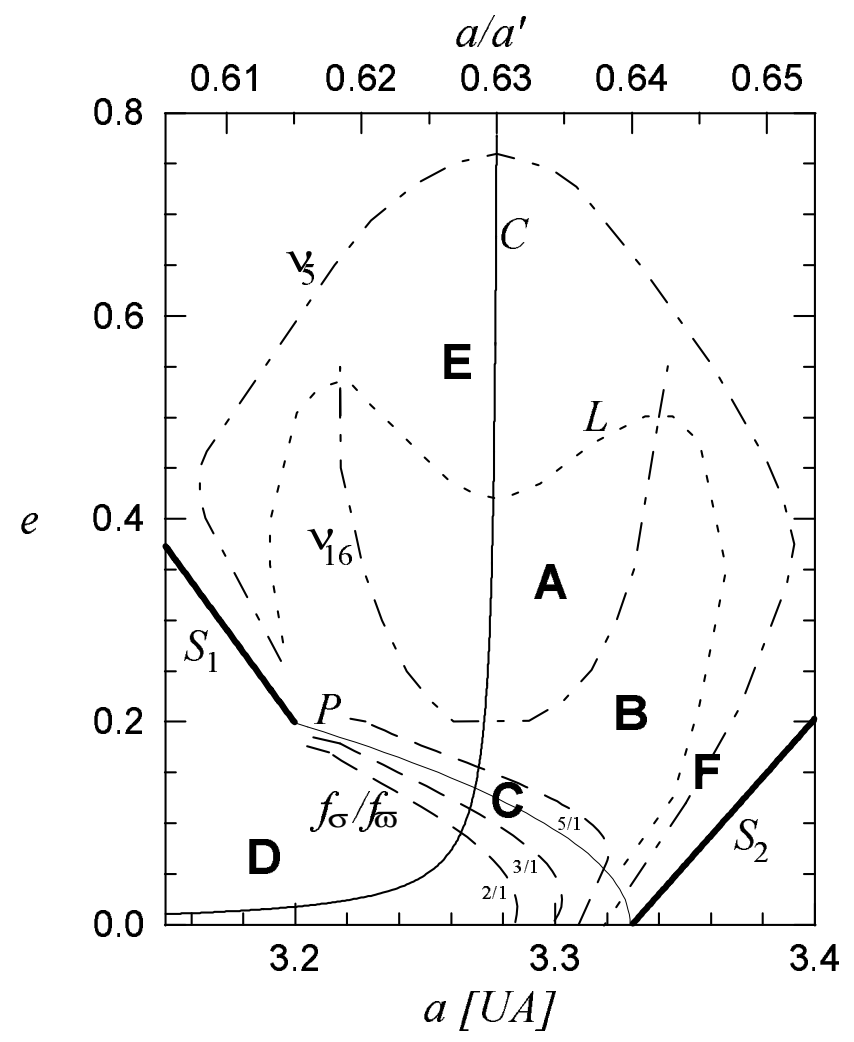

Figura A.3: Ressonância 2/1.

A região de libração para baixas excentricidades (região C) apresenta um comportamento caótico no âmbito do problema restrito elíptico plano. Esta caoticidade, que já foi observada por Giffen (1973), está vinculada ao "overlap" de ressonâncias secundárias na região (Henrard e Lemaitre, 1986; Lemaitre e Henrard 1990; Michtchenko e FerrazMello, 1995). No entanto, esta região fica isolada da região de altas excentricidade por uma faixa muito robusta de movimentos regulares (Ferraz-Mello, 1994b), e um mecanismo similar ao que esvazia a ressonância $3 / 1$ por difusão através de separatrizes não pode ocorrer na ressonância 2/1 no âmbito do problema elíptico plano.

A região de altas excentricidades (região E na Fig. A.3) está dominada pela exis${ }^{3} f_{\sigma}$ é a freqüência de libração do argumento crítico; $f_{\varpi}, f_{\Omega}$ são as freqüências de circulação do periélio e do nodo do asteróide. 
tência de ressonâncias seculares (pontos de corrotação) e foi estudada por Morbidelli e Moons (1993). Eles acharam que, no caso plano, o "overlap" das ressonâncias $\nu_{5}$ e $\nu_{6}$ gera uma região de movimentos caóticos muito grande. Quando a terceira dimensão é levada em conta, a dinâmica é dominada pelas librações do argumento pericêntrico $\omega$, isto é, a ressonância de Kozai. O "overlap" desta ressonância com $\nu_{5}$ e $\nu_{6}$ gera uma vasta região de movimentos caóticos onde o asteróide é alternadamente capturado pelas ressonâncias seculares e as librações de $\omega$. Henrard et al. (1995) chamam esta região de complexo de ressonâncias pericêntricas. Em outras ressonâncias, como a 3/1 e a 5/2, esta região abrange uma boa parte do espaço, e a difusão caótica gerada pelo "overlap" das ressonâncias seculares resulta ser um mecanismo de esvaziamento muito eficiente em escalas de tempo curtas (Klafke et al., 1992; Moons e Morbidelli, 1995).

A região em torno das separatrizes da ressonância (região F na Fig. A.3) apresenta certas particularidades. Michtchenko e Ferraz-Mello (1995) mostram que os objetos nessa região têm uma dinâmica muito complexa. Existe uma faixa de movimentos caóticos vinculada ao "overlap" da ressonância $\nu_{5}$ e $f_{\varpi} / f_{\Omega}$, que é a extensão do complexo pericêntrico nas baixas excentricidades. Aparece também uma faixa de movimentos regulares para excentricidades intermédias, onde $\sigma$ e o argumento $\varpi-\varpi$ Jup libram simultaneamente em torno de $0^{\circ}$. Este comportamento já fora identificado por Morbidelli e Moons (1993) usando um modelo semi-analítico. Finalmente, aqueles objetos situados inicialmente muito perto da separatriz ficam alternando seu movimento entre librações pericêntricas e circulações apocêntricas.

É bem conhecido o fato de que as barreiras de movimentos regulares que separam a região das ressonâncias secundárias da região das ressonâncias seculares sobrevivem no problema plano, mesmo levando em conta as perturbações de longo período na órbita de Júpiter. Foi Wisdom (1987) quem propôs pela primeira vez que um possível mecanismo de esvaziamento da região das ressonâncias secundárias (C) na ressonância $2 / 1$ podia estar relacionado à difusão na terceira dimensão. Estudos analíticos mostraram mais tarde (Henrard et al., 1995) que, efetivamente, os objetos desta região podem difundir caoticamente na inclinação até atingir uma "ponte" para $I \sim 30^{\circ}$, que lhes permite passar à região caótica das ressonâncias pericêntricas (E), onde os objetos são rapidamente ejetados. No plano $a, e$, este mecanismo pode ser visualizado como uma difusão rápida através das ressonâncias secundárias e uma difusão lenta ao longo destas ressonâncias, 
acompanhada de um aumento na inclinação. Desta forma, os objetos atingem a região F (que se fecha sobre o ramo pericêntrico nas altas inclinações) e a partir dali são levados à região das altas excentricidades.

A região de excentricidades intermédias em torno do ramo pericêntrico, entre a curva $L$ e a ressonância $\nu_{16}$ (região A na Fig. A.3), apresenta um comportamento totalmente diferente. Esta região está associada a uma teia de ressonâncias seculares de muito alta ordem e comporta-se como uma região extremamente estável. Integrações numéricas de Morbidelli (1996) e de Ferraz-Mello e Michtchenko (1996) por intervalos de tempo de $\sim 10^{8}-10^{9}$ anos, mostram um comportamento muito regular e não parece possível que estes objetos possam ser levados a colidir com outros planetas, exceto que um mecanismo de difusão lenta atue em escalas de tempo extremamente grandes. Em particular, Ferraz-Mello (1994b) determinou a existência de uma estocasticidade global dentro da ressonância $2 / 1$ que pode contribuir fortemente para esvaziar essa região em escalas de tempo da ordem da idade do Sistema Solar. Esta escala de tempo seria muito maior no caso da ressonância $3 / 2$, razão pela qual ainda existe um grande número de objetos nessa ressonância. Uma pesquisa recente e muito detalhada realizada por Nesvorný e Ferraz-Mello (1997a,b) utilizando o mapa freqüencial (Laskar et al., 1992; Šidlichovský e Nesvorný, 1997) mostrou que efetivamente, as taxas de difusão são, em média, uma ordem de magnitude maiores na ressonância $2 / 1$ do que nas ressonâncias $3 / 2$ e $4 / 3$. Destaquemos o fato de que estes resultados baseados no mapa freqüencial são muito mais precisos e confiáveis do que os resultados de outros estudos similares baseados no cálculo de expoentes de Lyapunov.

O limite inferior da região A é definido pela ressonância $\nu_{16}$. Ao longo desta ressonância, as taxas de difusão obtidas com o mapa freqüencial são muito altas. Os objetos sobre a $\nu_{16}$ apresentam, em geral, um aumento significativo na inclinação e uma difusão ao longo desta ressonância que os leva até a região do complexo pericêntrico nas altas excentricidades. Michtchenko e Ferraz-Mello (1997) apresentam resultados numéricos que mostram que existe uma teia de ressonâncias seculares de alta ordem na região $A$, que pode ser responsável pela difusão na região (através e ao longo destas ressonâncias), e pelo aumento da amplitude de libração, que leva os objetos a atingirem a ressonância $\nu_{16}$, causando o subseqüente escape dos mesmos.

A partir dos resultados do mapeamento simplético plano de Ferraz-Mello (1997), e 
de experimentos numéricos detalhados de Michtchenko e Ferraz-Mello (1997), sabe-se que a região $A$ é muito sensível às variações da freqüência do ângulo $5 \lambda_{\text {Sat }}-2 \lambda_{\text {Jup }}$, associado à perturbação de longo período introduzida pela quase-ressonância $5 / 2$ entre Júpiter e Saturno. Quando o valor desta freqüência bate com o valor da freqüência de libração na ressonância $\left(f_{\sigma}\right)$, os processos difusivos na região são acelerados significativamente, podendo levar aos objetos a escapar em escalas de tempo de $\sim 10^{8}$ anos ou menores. Na verdade, o valor real da freqüência do termo $5 / 2$ é aproximadamente metade do valor de $f_{\sigma}$ (880 anos de período e 440 anos, respectivamente), mas uma variação de só $0.1 \%$ no semieixo de Saturno é suficiente para duplicar esse valor. Podemos então supor que a evolução secular de Júpiter e Saturno em tempos passados poderia ter levado a uma configuração temporária na qual $f_{\sigma} \approx 5 n_{\text {Sat }}-2 n_{\text {Jup }}$, contribuindo ao rápido esvaziamento da região A. Uma característica importante do comportamento dos objetos nesta região é que as librações apresentam uma estrutura muito complexa, com um espectro de freqüências próprias que possui um grande número de linhas de amplitude similar e que correspondem a harmônicos da forma $f_{\sigma}+k f_{\varpi}(k$ inteiro). (Michtchenko e Ferraz-Mello, 1995, 1996). Portanto, mesmo que a freqüência $5 n_{\text {Sat }}-2 n_{\text {Jup }}$ não bata exatamente com $f_{\sigma}$, ela pode vir a bater facilmente com algum destes harmônicos, gerando um efeito similar.

Outra região que apresenta um comportamento bastante regular é a região de excentricidades intermédias e amplitudes de libração de $\sigma$ grandes $\left(\sim 60^{\circ}-90^{\circ}\right)$, indicada como região B na Fig. A.3. É nessa região onde acha-se a maior parte dos objetos reais conhecidos na ressonância. Os resultados do mapa freqüencial (Nesvorný e Ferraz-Mello, 1997a,b) mostram que esta é a região da ressonância onde a velocidade de difusão é mais baixa, e isto acontece particularmente para $e<0.3$. Quando olhamos a terceira dimensão, percebemos que esta região de estabilidade em excentricidades mais baixas começa a fechar-se, e praticamente desaparece para $I \sim 30^{\circ}$. Para inclinações maiores existe um "overlap" generalizado entre as ressonâncias secundárias, a $\nu_{16}$, e o complexo pericêntrico. Por outro lado, a velocidade de difusão cresce um pouco para $e>0.3$. Nesta região de excentricidades mais altas, existem uns poucos asteróides com comportamento caótico, entre os quais podemos mencionar 1362 Griqua e 3688 Navajo, este último muito próximo à ressonância $\nu_{16}$.

Bem no meio da região $\mathrm{B}$, entre a ressonância $\nu_{16}$ e a região das ressonâncias se- 
cundárias, ou seja, para $e<0.3$, a regularidade é notória. Nessa região encontra-se um pequeno grupo de asteróides com boas observações (numerados e multi-oposicionais), cujo principal exemplo é 3789 Zhongguo (cf. Fig. 5.1, Tabela 5.1). Estudos numéricos mostram que o comportamento destes objetos é muito regular e que podem ficar na região por intervalos de tempo muito longos. Segundo Morbidelli et al. (1995), eles poderiam ser um resíduo do processo colisional que dera origem à família de Themis. Na periferia desta região estável, o comportamento das órbitas é caótico, porém vários asteróides reais, em geral com órbitas determinadas provisoriamente, podem ser achados ali (cf. Michtchenko e Ferraz-Mello, 1997).

Finalmente, uma última região de interesse é a região das circulações pericêntricas para muito baixas excentricidades, indicada como região D na Fig. A.3. Os resultados do mapa freqüencial mostram esta região como sendo muito estável (significativamente mais estável do que a região B); no entanto ela está bastante despovoada de objetos. Sobre esta região não existem pesquisas detalhadas. Não possui associada uma teia de ressonâncias conhecida e não parece estar conectada com nenhuma das outras regiões da ressonância. O que acontece nesta região é ainda um problema a ser estudado.

\section{A.2.2. A ressonância $3 / 2$}

A Fig. A.4 apresenta o ramo pericêntrico $(C)$ e as separatrizes $\left(S_{1}, S_{2}\right)$ da ressonância $3 / 2$, junto com algumas das ressonâncias seculares e secundárias. Novamente, a curva contínua $P$ representa o limite inferior da região de libração e a curva pontilhada $L$ é o limite inferior das separatrizes das ressonâncias seculares. É interessante perceber que as ressonâncias secundárias (região C) ocupam aproximadamente a mesma região que no caso da ressonância 2/1, enquanto que as ressonâncias seculares (região E) situam-se em excentricidades menores que no caso da citada ressonância. Também é interessante destacar que a ressonância $\nu_{16}$ (não mostrada na Fig. A.4) fica por acima da curva $L$. Assim, as regiões A e B que apareciam diferenciadas na ressonância 2/1, ficam, no caso da 3/2, reduzidas a só uma região, indicada na Fig. A.4 como AB.

As órbitas caóticas na região das ressonâncias secundárias (C) são menos numerosas do que no caso da ressonância 2/1. Michtchenko e Ferraz-Mello (1995) explicam esta diferença a partir do fato de que, na ressonância $3 / 2$, as ressonâncias secundárias não apresentam "overlap" entre elas nas baixas inclinações como acontecia na 2/1. Por 


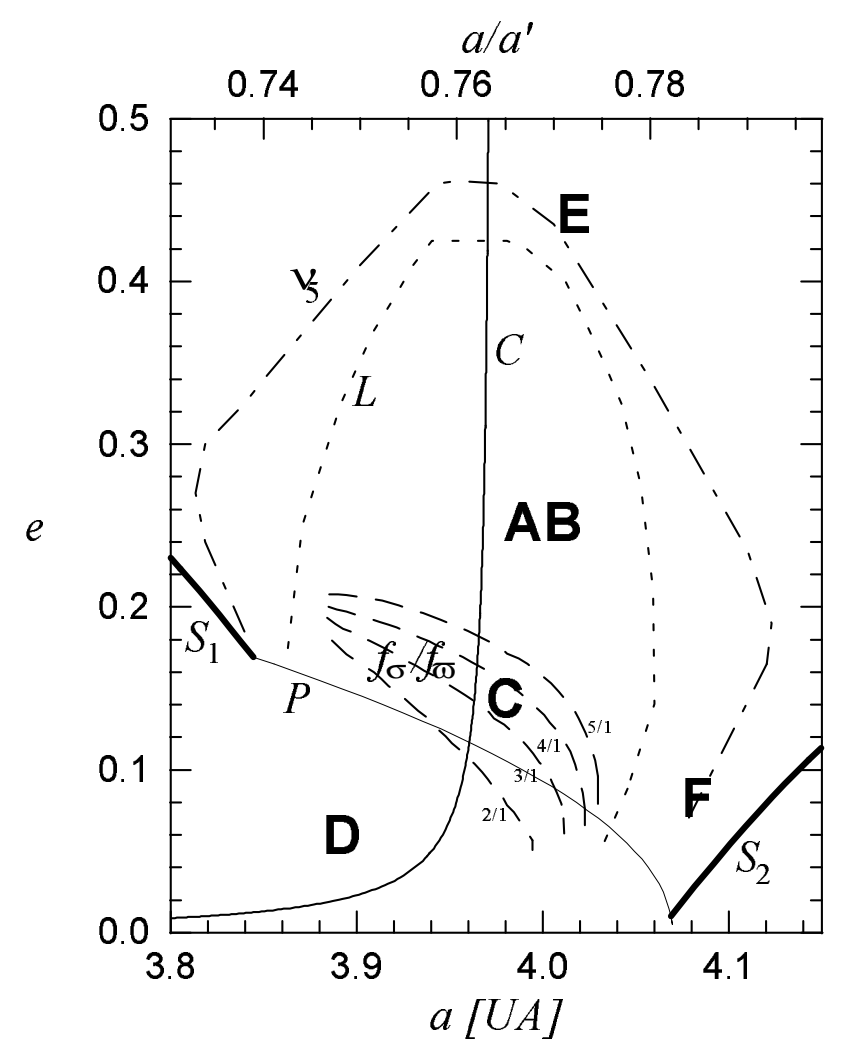

Figura A.4: Ressonância 3/2.

outro lado, quando aumenta a inclinação, este "overlap" começa a acontecer e para valores $I \sim 20^{\circ}$ toda a região C torna-se fortemente caótica (Michtchenko e FerrazMello, 1996).

A região do complexo pericêntrico (E) apresenta as mesmas características que no caso da ressonância 2/1. O "overlap" das ressonâncias seculares gera uma vasta região de movimentos fortemente caóticos para $e>0.4$, e com grandes variações na excentricidade.

A região perto das separatrizes (região F) está associada a uma região fortemente caótica, como no caso da ressonância 2/1. Isto evidencia-se claramente através do mapa freqüencial (Nesvorný e Ferraz-Mello, 1997a,b) e dos experimentos numéricos de Michtchenko e Ferraz-Mello (1995), que mostram que os objetos na região são rapidamente ejetados do cinturão de asteróides. As librações em torno de $0^{\circ}$ do argumento $\varpi-\varpi_{\text {Jup }}$ ficam cercadas por uma vasta região de movimentos caóticos (Morbidelli e Moons, 1993). 
$A$ região $A B$ comporta-se como sendo extremamente regular, particularmente em torno do ramo pericêntrico para amplitudes de libração $<90^{\circ}$. É precisamente nessa região que se encontram a maioria dos asteróides do grupo Hilda (uns 80 objetos). O cálculo de tempos de Lyapunov mostra que esses objetos devem sobreviver à fraca estocasticidade da região por intervalos de tempo maiores do que a idade do Sistema Solar. O fato de que a ressonância secular $\nu_{16}$ não esteja presente nesta região faz com que não apareçam os comportamentos difusivos rápidos na inclinação que eram observados na mesma região na ressonância 2/1. Se olhamos na terceira dimensão, esta região de estabilidade fecha-se nas altas inclinações formando um triângulo cujo vértice superior acha-se em $I>30^{\circ}$. Acima deste limite aparece uma vasta região de movimentos estocásticos que se une à já mencionada região caótica das ressonâncias secundárias nas altas inclinações. Isto explica o fato de que exista um limite na distribuição em inclinação dos Hildas (aproximadamente $20^{\circ}$ ).

Ao contrario do que acontecia na ressonância 2/1, as librações dos objetos nesta região são extremamente quase-periódicas, e o espectro de freqüências próprias dos elementos orbitais apresenta muito poucas linhas; em geral, só uma linha de grande amplitude bem isolada e alguns harmônicos superiores de amplitude desprezível (Michtchenko e Ferraz-Mello, 1995, 1996). Isto faz com que seja muito mais difícil ocorrer um batimento exato entre a freqüência de libração e a freqüência $5 n_{\text {Sat }}-2 n_{\text {Jup }}$. Note-se que $f_{\sigma}$ é umas três vezes maior do que $5 n_{\text {Sat }}-2 n_{\text {Jup }}$ (270 anos de período contra 880 anos, respectivamente).

Finalmente, a região das circulações pericêntricas (região D) possui características diferentes da sua análoga na ressonância 2/1. Esta região apresenta uma caoticidade moderada, que se manifesta claramente nas taxas de difusão fornecidas pelo mapa freqüencial. Existe um pequeno grupo de aproximadamente 5 objetos na região que alternam entre circulação e libração de $\sigma$, e cujo principal representante é 334 Chicago. Resultados obtidos com o mapeamento simplético plano de Ferraz-Mello (1997) mostram que estes objetos são expulsos rapidamente da ressonância.

Com isto completamos o panorama geral do que é conhecido na atualidade sobre a dinâmica das ressonâncias $2 / 1$ e $3 / 2$. 


\section{APÊNDICE B}

\section{LISTA DOS ASTERÓIDES REAIS UTILIZADOS}

Apresentamos neste apêndice as tabelas contendo a informação sobre os objetos reais que foram testados na ressonância 3/2. Estes objetos foram tomados da base de dados de Bowell et al. (1994). Levando em conta só aqueles asteróides numerados e os não numerados mas com pelo menos 20 observações em múltipla oposição, achamos um total de 82 objetos no intervalo 3.80-4.15 UA. A Tabela I apresenta os valores do semieixo $a$, excentricidade $e$, e inclinação $I$, respeito da eclíptica, como também os valores dos ângulos ressonantes $\psi=3 \lambda_{\mathrm{J}}-2 \lambda$ e $\sigma=\psi-\varpi$, e do argumento de periélio $\omega=\sigma_{z}-\sigma$ destes objetos. Estes dados estão calculados para JD 2450500.5, e foram utilizados como condições iniciais para as iterações com o mapeamento. A seguir fornecemos os valores dos elementos orbitais de Júpiter e da obliqüidade da eclíptica para a mesma data:

$$
\begin{aligned}
a & =5.2313885 \mathrm{UA} \\
e & =0.0495813 \\
I & =1.3045084^{\circ} \\
\lambda & =307.6000110^{\circ} \\
\varpi & =11.4273413^{\circ} \\
\Omega & =100.3677313^{\circ} \\
\epsilon & =23.4371928^{\circ}
\end{aligned}
$$


TABELA I

Condições iniciais dos asteróides na ressonância $3 / 2$

\begin{tabular}{|c|c|c|c|c|c|c|c|}
\hline $\mathrm{N}^{\circ}$ & Nome & $a$ [U.A.] & $e$ & $I\left[^{\circ}\right]$ & $\psi\left[^{\circ}\right]$ & $\sigma\left[^{\circ}\right]$ & $\omega\left[^{\circ}\right]$ \\
\hline 153 & Hilda & 3.9802206 & 0.1436683 & 7.8325310 & -108.3610800 & -19.5839250 & 42.7657780 \\
\hline 190 & Ismene & 3.9798445 & 0.1658309 & 6.1641640 & 77.6193470 & -12.6592090 & 274.1984700 \\
\hline 334 & Chicago & 3.8734271 & 0.0427414 & 4.6580650 & 170.0730100 & -97.7883520 & 137.1185900 \\
\hline 361 & Bononia & 3.9448908 & 0.2137855 & 12.6612120 & 67.3221010 & -22.1906480 & 70.2695880 \\
\hline 499 & Venusia & 4.0099159 & 0.2136125 & 2.0898180 & 62.8505910 & -10.5717450 & 176.7409500 \\
\hline 748 & Simeisa & 3.9352994 & 0.1889566 & 2.2577520 & 88.5080510 & 2.3643863 & 180.2470000 \\
\hline 958 & Asplinda & 3.9695746 & 0.1867761 & 5.6483370 & 51.0101010 & -28.5791500 & 95.7664880 \\
\hline 1038 & Tuckia & 3.9589083 & 0.2313045 & 9.2255810 & -53.5146790 & -58.3447910 & 306.4679500 \\
\hline 1162 & Larissa & 3.9292399 & 0.1098849 & 1.8895140 & -106.8214500 & 0.6487863 & 212.6775200 \\
\hline 1180 & Rita & 3.9960966 & 0.1593616 & 7.1983930 & -84.4742890 & -22.9968220 & 210.0525300 \\
\hline 1202 & Marina & 3.9773861 & 0.1807047 & 3.3621230 & -66.1935030 & -67.1199630 & 310.2449100 \\
\hline 1212 & Francette & 3.9515930 & 0.1875589 & 7.5869560 & 164.5549100 & 24.4839270 & 350.3341100 \\
\hline 1256 & Normannia & 3.8995767 & 0.0867009 & 4.1764810 & -4.2429987 & 5.3313653 & 111.8604700 \\
\hline 1268 & Libya & 3.9593027 & 0.1036716 & 4.4242550 & 96.1327950 & -21.9083140 & 126.1700700 \\
\hline 1269 & Rollandia & 3.8997472 & 0.0972988 & 2.7569380 & -179.7480100 & 19.5337410 & 25.8235460 \\
\hline 1345 & Potomac & 3.9822945 & 0.1783742 & 11.3908830 & 125.8827300 & 12.6420280 & 335.5899000 \\
\hline 1439 & Vogtia & 3.9934067 & 0.1135285 & 4.2017690 & 146.9392300 & 3.7930593 & 107.1212900 \\
\hline 1512 & Oulu & 3.9423732 & 0.1515686 & 6.4993840 & -158.4417700 & -49.4325920 & 240.6294000 \\
\hline 1529 & Oterma & 4.0041313 & 0.1916222 & 9.0306450 & 80.1204130 & 41.7655110 & 297.3346400 \\
\hline 1578 & Kirkwood & 3.9241518 & 0.2377630 & 0.8121990 & 118.9319300 & 41.6066530 & 3.3849150 \\
\hline 1746 & Brouwer & 3.9594876 & 0.2053468 & 8.3808350 & 36.9692270 & 24.4984110 & 49.9019350 \\
\hline 1748 & Mauderli & 3.9362147 & 0.2287656 & 3.2967720 & -79.3084910 & -45.3497880 & 200.0069700 \\
\hline 1754 & Cunningham & 3.9566094 & 0.1686509 & 12.1127050 & -60.2198470 & 23.9971150 & 112.4895500 \\
\hline 1877 & Marsden & 3.9484046 & 0.2106004 & 17.5459060 & -77.6601910 & -18.1428260 & 307.5235300 \\
\hline 1902 & Shaposhnikov & 3.9819880 & 0.2237336 & 12.4869490 & -35.8143410 & -4.5941407 & 269.0835900 \\
\hline 1911 & Schubart & 3.9779602 & 0.1656133 & 1.6508930 & 134.8471300 & 25.6114120 & 183.6385800 \\
\hline 1941 & Wild & 3.9773928 & 0.2833861 & 3.9532470 & 46.5900610 & 41.9188190 & 303.9112400 \\
\hline 2067 & Aksnes & 3.9538092 & 0.1830562 & 3.0697390 & 80.5033610 & -10.0017350 & 299.8192700 \\
\hline 2246 & Bowell & 3.9420061 & 0.0962507 & 6.4941040 & 162.3629000 & -22.5298990 & 28.9622310 \\
\hline 2312 & Duboshin & 3.9739145 & 0.1471311 & 5.1894350 & 88.3472710 & 40.5006560 & 345.7996400 \\
\hline 2483 & Guinevere & 3.9758277 & 0.2725694 & 4.4951160 & 110.4845600 & 34.0813980 & 184.0548400 \\
\hline 2624 & Samitchell & 3.9615739 & 0.1200194 & 2.7724850 & -50.9944970 & 1.7565063 & 145.0814000 \\
\hline 2760 & Kacha & 3.9778528 & 0.1206802 & 13.4660900 & 116.6999500 & -31.7048990 & 155.5074200 \\
\hline 2959 & Scholl & 3.9398700 & 0.2741980 & 5.2310510 & 36.5627370 & -11.0076310 & 286.0462200 \\
\hline 3134 & Kostinsky & 3.9740357 & 0.2201524 & 7.6275570 & 46.9712210 & -15.7474320 & 165.3924900 \\
\hline 3202 & Graff & 3.9493065 & 0.1014357 & 11.0405050 & -164.0544700 & 78.6148840 & 271.7552300 \\
\hline 3254 & Bus & 3.9701752 & 0.1715034 & 4.4419570 & -60.8168310 & -50.7934120 & 306.0486600 \\
\hline 3290 & Azabu & 3.9671843 & 0.1249151 & 2.7706430 & -137.6633300 & 34.1098460 & 112.9313900 \\
\hline
\end{tabular}


TABela I: (continuação)

\begin{tabular}{|c|c|c|c|c|c|c|c|}
\hline $\mathrm{N}^{\circ}$ & Nome & $a[\mathrm{U} . \mathrm{A}]$. & $e$ & $I\left[^{\circ}\right]$ & $\psi\left[^{\circ}\right]$ & $\sigma\left[^{\circ}\right]$ & $\omega\left[^{\circ}\right]$ \\
\hline 3415 & Danby & 3.9651498 & 0.2466262 & 1.3553840 & 41.3998610 & 9.0523103 & 137.7118900 \\
\hline 3514 & $1971 \mathrm{UJ}$ & 3.9400180 & 0.2017822 & 3.5155590 & -29.8931230 & -39.0101190 & 344.2148200 \\
\hline 3557 & Sokolsky & 4.0114503 & 0.1759717 & 6.0308810 & -74.8113490 & -26.4212570 & 125.1663900 \\
\hline 3561 & Devine & 3.9662036 & 0.1333186 & 9.6553940 & -72.3558510 & -11.8488930 & 181.3177700 \\
\hline 3571 & Milanstefanik & 3.9356793 & 0.1198222 & 7.8312510 & -116.9395800 & -31.5421680 & 25.2300790 \\
\hline 3577 & Putilin & 3.9597927 & 0.1870366 & 3.7412440 & 167.9799300 & 66.3253140 & 180.7530100 \\
\hline 3655 & Eupraksia & 4.0002548 & 0.2092608 & 3.8496900 & -36.5482350 & -57.3808600 & 42.2595850 \\
\hline 3694 & Sharon & 3.9475443 & 0.1957160 & 4.9542410 & 93.5193750 & 59.5063600 & 82.1592210 \\
\hline 3843 & OISCA & 4.0017559 & 0.1275964 & 3.9295590 & 114.0726800 & 57.9469880 & 26.6343290 \\
\hline 3923 & Radzievskij & 3.9524372 & 0.2204746 & 3.4854830 & 75.3278670 & -2.1334567 & 285.0151200 \\
\hline 3990 & Heimdal & 3.9415038 & 0.2409480 & 9.4998140 & 49.3149410 & 16.8133340 & 199.5847600 \\
\hline 4196 & Shuya & 3.9398868 & 0.0540817 & 1.4789050 & -62.0378390 & -67.8638950 & 198.4729100 \\
\hline 4230 & van den Bergh & 3.9468171 & 0.1322398 & 3.0965050 & -158.3022400 & 17.1710340 & 23.9118470 \\
\hline 4255 & Spacewatch & 3.9666312 & 0.1550923 & 2.6073130 & -142.6233500 & -42.6677130 & 76.0010860 \\
\hline 4317 & Garibaldi & 3.9902135 & 0.1580137 & 9.8086570 & 146.0824600 & 9.4922203 & 97.7415720 \\
\hline 4446 & Carolyn & 3.9814193 & 0.2847321 & 7.2410800 & -91.5702670 & -38.6513090 & 117.7106800 \\
\hline 4495 & $1988 \mathrm{VS}$ & 3.9246312 & 0.1587848 & 5.2606190 & 31.7945750 & -46.9697640 & 214.3267600 \\
\hline 4757 & Liselotte & 3.9392515 & 0.0810811 & 0.1765480 & -160.0538600 & 20.5146160 & 93.3785840 \\
\hline 5368 & 1984 SW5 & 3.9568429 & 0.0818915 & 6.2382600 & 136.4482800 & -11.7571020 & 348.2708700 \\
\hline 5439 & 1990 RW & 3.9815120 & 0.1470358 & 1.2607900 & 15.9372490 & 48.8226220 & 334.2532500 \\
\hline 5603 & Rausudake & 3.9591023 & 0.0660520 & 4.3297390 & 146.6153100 & -70.1246130 & 19.7956280 \\
\hline 5661 & Hildebrand & 3.9535540 & 0.2412253 & 13.3188730 & -87.2718350 & -47.3752730 & 2.6391310 \\
\hline 5711 & $1978 \mathrm{SO} 4$ & 3.9469013 & 0.1678561 & 6.3733880 & -52.1913150 & -5.7531307 & 298.7685100 \\
\hline 5928 & Pindarus & 3.9765680 & 0.1232601 & 9.2733510 & -107.0465100 & -21.1855870 & 109.6626300 \\
\hline 6124 & Mecklenburg & 3.9731583 & 0.2434268 & 9.3874330 & 21.0503390 & -23.9113040 & 244.8055800 \\
\hline 6237 & Chikushi & 3.9542390 & 0.0680312 & 5.3953450 & -81.3722250 & 34.1247940 & 218.1202000 \\
\hline 6984 & $1994 \mathrm{AO}$ & 3.9653275 & 0.1862866 & 16.8120180 & 93.5802090 & -3.1397237 & 250.1352800 \\
\hline 7027 & $1993 \mathrm{XT}$ & 3.9539176 & 0.1949148 & 12.4038200 & 80.8072130 & -11.9421420 & 15.3319430 \\
\hline 7174 & $1988 \mathrm{SQ}$ & 3.9802039 & 0.1833343 & 12.7012900 & -138.4973700 & -65.2424110 & 291.9123400 \\
\hline 7284 & $1989 \mathrm{VW}$ & 3.9925486 & 0.1550058 & 6.3521940 & 133.2913900 & 43.2444790 & 42.2605010 \\
\hline 7394 & $1985 \mathrm{QX} 4$ & 3.9276687 & 0.0514185 & 8.6814860 & -118.7317300 & -105.8264600 & 171.2168700 \\
\hline \multirow[t]{7}{*}{7458} & 1984 DE1 & 3.8271552 & 0.1369919 & 1.8081230 & 40.9813890 & -161.4031200 & 235.4920500 \\
\hline & 1976 DJ1 & 3.9852859 & 0.1357123 & 5.9758550 & 162.5113200 & -47.5060200 & 95.9999930 \\
\hline & 1987 RO5 & 3.9471817 & 0.2908974 & 6.8143700 & 44.3193370 & 28.9184640 & 216.8480300 \\
\hline & 1988 VS6 & 3.9502102 & 0.2382294 & 4.9036960 & 82.1316290 & 20.9747040 & 211.6771500 \\
\hline & 1992 OZ9 & 3.9768774 & 0.2053486 & 3.5299860 & -103.4630700 & -53.5242660 & 259.4542500 \\
\hline & 1992 SU21 & 3.9679387 & 0.2317600 & 6.8623140 & 126.4255400 & 58.7490200 & 50.3878580 \\
\hline & 1993 PZ2 & 3.9425963 & 0.2636015 & 4.8481300 & -58.7902130 & -39.7154090 & 119.9721100 \\
\hline
\end{tabular}


TABela I: (continuação)

\begin{tabular}{llrrrrrr}
\hline \hline $\mathrm{N}^{\circ}$ & Nome & $a$ [U.A.] & \multicolumn{1}{c}{$I\left[^{\circ}\right]$} & \multicolumn{1}{c}{$\psi\left[^{\circ}\right]$} & \multicolumn{1}{c}{$\sigma\left[^{\circ}\right]$} & $\omega\left[^{\circ}\right]$ \\
\hline & & & & & & \\
$1994 \mathrm{AJ} 3$ & 3.9460689 & 0.1360260 & 4.4106620 & 99.1993550 & -19.7645060 & 317.1420100 \\
& $1994 \mathrm{PV} 24$ & 3.9470772 & 0.2611645 & 2.8828470 & 5.4038553 & 3.9943923 & 337.6851400 \\
& & & & & & \\
$1995 \mathrm{YK} 3$ & 3.9360612 & 0.0551868 & 3.6545980 & -140.7271400 & -7.7696677 & 341.0369400 \\
$1996 \mathrm{UC} 3$ & 4.0008488 & 0.2292000 & 4.2967650 & 86.9514950 & 40.6880640 & 103.6654100 \\
$1996 \mathrm{XR} 1$ & 3.9681868 & 0.2159451 & 4.6251070 & 32.8102390 & 19.2312110 & 285.7285900 \\
$4216 \mathrm{~T}-2$ & 3.9596494 & 0.1527959 & 9.2996010 & -134.9355900 & -88.0271140 & 143.8051300 \\
& & & & & & \\
\hline \hline
\end{tabular}




\begin{abstract}
ANEXO I
THE HIGH-ECCENTRICITY ASYMMETRIC EXPANSION OF THE DISTURBING FUNCTION FOR NON-PLANAR RESONANT PROBLEMS
\end{abstract}

O manuscrito que apresentamos neste anexo contém uma série de Apêndices que, por razões de espaço, não foram incluídos na versão publicada em Astronomy and Astrophysics. Mesmo não sendo indispensáveis, estes Apêndices dão completitude ao trabalho e facilitam a programação de um código para o cômputo do desenvolvimento assimétrico. 



\title{
The high-eccentricity asymmetric expansion of the disturbing function for non-planar resonant problems
}

\author{
F. Roig ${ }^{1}$, A. Simula ${ }^{1}$, S. Ferraz-Mello ${ }^{1}$, and M. Tsuchida ${ }^{2}$ \\ 1 Instituto Astronômico e Geofísico. Universidade de São Paulo Av. Miguel Stéfano 4200, São Paulo, SP, Brasil \\ 2 Inst. de Biociências, Letras e Ciências Exatas. Universidade Estadual Paulista São José do Rio Preto, SP, Brasil
}

Received 21 May 1997 / Accepted 22 August 1997

\begin{abstract}
In this paper we present an extension to the nonplanar case of the asymmetric expansion of the averaged resonant disturbing function of Ferraz-Mello \& Sato (1989, A\&A 225, 541-547). Comparions with the exact averaged disturbing function are also presented. The expansion gives a good approximation of the exact function in a wide region around the center of expansion.
\end{abstract}

Key words: celestial mechanics - solar system: general - methods: analytical

\section{Introduction}

An important challenge in dynamical astronomy is to find a suitable expansion of the disturbing function $R$ for a given problem. As far as planetary theory is concerned, the Laplace's series are widely used. They basically represent an expansion around coplanar circular orbits. However, these series have generally a poor convergence for high eccentricities and inclinations (see Ferraz-Mello, 1994 for a discussion).

A typical example where the Laplacian expansion can not be used because of its lack of convergence is that of the first-order resonances in the asteroidal main belt (Murray, 1986). In the case of the $2 / 1$ and $3 / 2$ resonances, the radius of convergence in eccentricity of the Laplace's expansion is 0.2 and 0.09 , respectively. Recall that both the Griquas (at 2/1) and the Hildas (at 3/2) have eccentricities higher than these values.

It was Woltjer (1928) who first proposed to use a Taylor expansion of the disturbing function around a point other than the origin. There is no reason "a priori" to suppose that such an expansion will not have the same convergence problems of the Laplacian expansion when we are far away from the center of expansion. On the contrary, the larger the eccentricity of the center, the smaller the radius of convergence (see for example Wintner, 1941, §285-§299). However, it is clear that it will be

Send offprint requests to: F. Roig good enough to describe the dynamics of libration as far as the real motion remains close to the center of expansion.

The asymmetric expansion of Woltjer allowed him to make a good prediction of the libration period of the 3/4 resonance between Titan and Hyperion. Later on, Ferraz-Mello (1987) reformulated Woltjer's idea in the form of an analytic expansion in the framework of the restricted planar three-body problem for a first-order resonance. This work was extended for resonances of any order by Ferraz-Mello \& Sato (1989): We will refer hereafter to their article as FMS89.

The planar asymmetric expansion led to the high-eccentricity libration theory of the Hildas' motion (Ferraz-Mello, 1988; Gallardo \& Ferraz-Mello, 1995). It was also used to study the corotation solutions of first-order resonances (Ferraz-Mello, 1989; Ferraz-Mello et al., 1993) and, more recently, to formulate a formal analytic theory for the high-eccentricity smallamplitude librations at first-order resonances (Ferraz-Mello et al., 1997). Numerical applications of the asymmetric expansion also allowed Ferraz-Mello \& Klafke (1991) to discover the dynamics of very-high eccentricity libration in the asteroidal $3 / 1$ resonance, and Ferraz-Mello (1997), to construct a symplectic mapping for the $2 / 1$ and $3 / 2$ resonances.

However, it becomes more and more evident that planar models are not sufficient to explain all the properties observed in some resonant systems. The articles of Wisdom (1987) and Henrard et al. (1995) are good examples of this fact. Thus, in many cases, the inclusion of the third dimension in the models is necessary.

An important advance in this direction was already made by Moons (1993, 1994), who extended the basic equations of FMS89, and presented a method to calculate numerically the resonant averaged disturbing function at each point of the phase space for the non-planar model. This method allows us to have an exact representation of the disturbing function. Despite of the problem of the high computational cost of a mean at each point of the phase space, the method was successfully applied in many works (see for example Morbidelli \& Moons, 1993; Moons, 1994; Henrard et al., 1995). 
FMS89's equations were also extended by Tsuchida (see Oliveira, 1995) who derived an extension of the asymmetric expansion to the non-planar case in which the plane of motion of the disturbing body is kept fixed. The aim of this paper is to extend the equations of FMS89 to the construction of the spatial asymmetric expansion in terms of the inclinations of both the disturbed and the disturbing bodies with respect to a fixed reference plane. Since this expansion is a polynomial, and its coefficients are computed only once, it is much faster than the numerical calculation of the exact disturbing function. However, it approximates well the disturbing function only in a neighborhood of the center of expansion, and is only useful in the case of moderate amplitude librations. In the case of large amplitude librations, it should be better to use the expansion of Yokoyama (1994), which is an expansion in powers of $\left(e-e_{0}\right)$ combined with a Fourier expansion in the resonant angle $\sigma$. However, that expansion was only calculated in the frame of the planar problem.

In Sects. 2 to 6 we present the basic calculations to obtain the asymmetric expansion of the disturbing function. These calculations are extended in Sect. 7 to the case of an oblate potential of the primary body. Finally, in Sect. 8, we present a comparison with the exact averaged disturbing function calculated numerically. The last section is devoted to the conclusions.

\section{The disturbing function}

Let $a, e, I, \omega, \Omega$ be the orbital elements in their usual notation, and $v, u, \ell$ the true, eccentric and mean anomalies, respectively. We will use primed variables for the disturbing body and unprimed ones for the disturbed body. We define the resonant angular variables for a generic resonance $(p+q): p$ in the form

$$
\begin{aligned}
\sigma & =\frac{p+q}{q} \lambda^{\prime}-\frac{p}{q} \lambda-\varpi \\
\sigma^{\prime} & =\frac{p+q}{q} \lambda^{\prime}-\frac{p}{q} \lambda-\varpi^{\prime} \\
\sigma_{z} & =\frac{p+q}{q} \lambda^{\prime}-\frac{p}{q} \lambda-\Omega \\
\sigma_{z}^{\prime} & =\frac{p+q}{q} \lambda^{\prime}-\frac{p}{q} \lambda-\Omega^{\prime} \\
Q & =\frac{\lambda-\lambda^{\prime}}{q}
\end{aligned}
$$

where $\varpi=\omega+\Omega$ and $\lambda=\ell+\varpi$ (also $\varpi^{\prime}=\omega^{\prime}+\Omega^{\prime}$ and $\left.\lambda^{\prime}=\ell^{\prime}+\varpi^{\prime}\right)$. Recall that $Q$ is the so called synodic angle, which is a fast variable in comparison to the others.

Now, we write the disturbing function of the restricted 3body problem as

$R=\frac{\mathrm{G} m^{\prime}}{a^{\prime}}\left[f+f^{1}\right]$

where $\mathrm{G}$ is the gravitational constant, $m^{\prime}$ and $a^{\prime}$ are the perturber's mass and semimajor axis, and $f$ and $f^{1}$ are the direct and indirect parts, respectively, which we write as

$$
f=a^{\prime}\left(r^{2}+r^{\prime 2}-2 r r^{\prime} \cos S\right)^{-1 / 2}
$$

$f^{1}=-a^{\prime} \frac{r}{r^{\prime 2}} \cos S$

where $S$ is the angle between the position vectors $\mathbf{r}$ and $\mathbf{r}^{\prime}$. This disturbing function depends on the inclinations only through $\cos S$. Introducing the notation

$\eta=\sin \frac{I}{2}, \quad \eta^{\prime}=\sin \frac{I^{\prime}}{2}$

we can write

$$
\begin{aligned}
\cos S=( & \left.-\eta^{2}-\eta^{\prime 2}+\eta^{2} \eta^{\prime 2}\right) \\
& \times \cos \left((v-\sigma)-\left(v^{\prime}-\sigma^{\prime}\right)\right) \\
+ & 2 \eta \eta^{\prime} \sqrt{1-\eta^{2}} \sqrt{1-\eta^{\prime 2}} \\
& \times\left[\cos \left((v-\sigma)-\left(v^{\prime}-\sigma^{\prime}\right)+\sigma_{z}-\sigma_{z}^{\prime}\right)\right. \\
& \left.-\cos \left((v-\sigma)+\left(v^{\prime}-\sigma^{\prime}\right)+\sigma_{z}+\sigma_{z}^{\prime}\right)\right] \\
+ & \left(1-\eta^{2}\right) \eta^{\prime 2} \cos \left((v-\sigma)+\left(v^{\prime}-\sigma^{\prime}\right)+2 \sigma_{z}^{\prime}\right) \\
+ & \left(1-\eta^{\prime 2}\right) \eta^{2} \cos \left((v-\sigma)+\left(v^{\prime}-\sigma^{\prime}\right)+2 \sigma_{z}\right) \\
+ & \eta^{2} \eta^{\prime 2} \cos \left((v-\sigma)-\left(v^{\prime}-\sigma^{\prime}\right)+2 \sigma_{z}-2 \sigma_{z}^{\prime}\right)
\end{aligned}
$$

On the other hand, $R$ depends on the eccentricities through $r, r^{\prime}$ and $v, v^{\prime}$ which involves the solution of Kepler's equation. If we assume that the eccentricity of the perturber is small enough we can use the known elliptic expansions (Brouwer \& Clemence, 1961) and write

$$
\begin{aligned}
& r^{\prime}=a^{\prime}\left[1-e^{\prime} \cos \ell^{\prime}+\frac{1}{2} e^{2}\left(1-\cos 2 \ell^{\prime}\right)+\mathscr{O}\left(e^{\prime 3}\right)\right] \\
& v^{\prime}=\ell^{\prime}+2 e^{\prime} \sin \ell^{\prime}+\frac{5}{4} e^{\prime 2} \sin 2 \ell^{\prime}+\mathscr{O}\left(e^{\prime 3}\right)
\end{aligned}
$$

where, in terms of the variables of Eqs. (1), we have

$\ell^{\prime}=\sigma^{\prime}+p Q$

A similar assumption is not done in the case of the disturbed body and the dependence of $r, v$ on $e$ will be considered later (see Sect. 5).

Now, using Eqs. (5) and (6) we can write $R$ as a function of the form

$R=R\left(r, v, \sigma, \eta, \sigma_{z}, r^{\prime}\left(a^{\prime}, e^{\prime}, \ell^{\prime}\right), v^{\prime}\left(e^{\prime}, \ell^{\prime}\right), \sigma^{\prime}, \eta^{\prime}, \sigma_{z}^{\prime}\right)$

and expand it in Taylor series. We will do this in two steps, respectively discused in the next two sections.

\section{Expansion in $e^{\prime}, \eta^{\prime}$ and $\eta$}

Since $e^{\prime}$ is a small quantity we start with an expansion around $e^{\prime}=0$ up to $\odot\left(e^{\prime 2}\right)$ (this part of the expansion will lead to the same expressions that appear in FMS89). We stress the fact that such expansion is sufficient if we are looking for a first-order averaged expansion of $R$, on the hypothesis that $e^{\prime}<\mathscr{Q}\left(\sqrt{m^{\prime}}\right)$. We will also assume that $\eta^{\prime} \simeq \mathscr{Q}\left(e^{\prime}\right)$. Note that this assumption is valid for the inclinations of almost all the planets with respect 
to the invariant plane of the outer Solar System or with respect to the ecliptic. On the other hand, many asteroids have inclinations less than $30^{\circ}$ and we will assume $\eta \simeq \mathscr{Q}\left(\sqrt{e^{\prime}}\right)$. Thus, we will expand $R$ around $\eta^{\prime}=0$ and $\eta=0$ up to orders consistent with Q $\left(e^{2}\right)$.

Denoting $\mathscr{R}=f+f^{1}$ we write

$$
\begin{aligned}
R=\frac{\mathrm{G} m^{\prime}}{a^{\prime}}[ & \mathscr{R}_{0} \\
& +\frac{\partial \mathscr{R}}{\partial e^{\prime}} e^{\prime}+\frac{1}{2} \frac{\partial^{2} \mathscr{R}}{\partial e^{\prime 2}} e^{\prime 2}+\frac{\partial \mathscr{R}}{\partial \eta^{\prime}} \eta^{\prime}+\frac{1}{2} \frac{\partial^{2} \mathscr{R}}{\partial \eta^{\prime 2}} \eta^{\prime 2} \\
& +\frac{\partial \mathscr{R}}{\partial \eta} \eta+\frac{1}{2} \frac{\partial^{2} \mathscr{R}}{\partial \eta^{2}} \eta^{2}+\frac{1}{6} \frac{\partial^{3} \mathscr{R}}{\partial \eta^{3}} \eta^{3}+\frac{1}{24} \frac{\partial^{4} \mathscr{R}}{\partial \eta^{4}} \eta^{4} \\
& +\frac{\partial^{2} \mathscr{R}}{\partial e^{\prime} \partial \eta} e^{\prime} \eta+\frac{1}{2} \frac{\partial^{3} \mathscr{R}}{\partial e^{\prime} \partial \eta^{2}} e^{\prime} \eta^{2}+\frac{\partial^{2} \mathscr{R}}{\partial e^{\prime} \partial \eta^{\prime}} e^{\prime} \eta^{\prime} \\
& \left.+\frac{\partial^{2} \mathscr{R}}{\partial \eta^{\prime} \partial \eta} \eta^{\prime} \eta+\frac{1}{2} \frac{\partial^{3} \mathscr{R}}{\partial \eta^{\prime} \partial \eta^{2}} \eta^{\prime} \eta^{2}+\mathscr{O}\left(e^{\prime 3}\right)\right]
\end{aligned}
$$

and hereafter we assume that all the derivatives are evaluated at $e^{\prime}=\eta^{\prime}=\eta=0$. Since the expansion is symmetric with respect to the inclinations, the D'Alembert rule holds and, so, just the terms in the even powers of the inclinations should survive. Hence, we must have

$$
\frac{\partial \mathscr{R}}{\partial \eta^{\prime}}=\frac{\partial \mathscr{R}}{\partial \eta}=\frac{\partial^{3} \mathscr{R}}{\partial \eta^{3}}=\frac{\partial^{2} \mathscr{R}}{\partial e^{\prime} \partial \eta}=\frac{\partial^{2} \mathscr{R}}{\partial e^{\prime} \partial \eta^{\prime}}=\frac{\partial^{3} \mathscr{R}}{\partial \eta^{\prime} \partial \eta^{2}}=0 .
$$

The remaining derivatives are not null, and in order to calculate them we divide $\mathscr{R}$ in its direct and indirect parts.

First, we introduce the notation $c=\cos S$ and

$$
b=\frac{r^{2}+r^{\prime 2}-2 r r^{\prime} c}{a^{\prime 2}}
$$

Thus for the direct part we can write

$$
\begin{aligned}
f_{0} & =b_{0}^{-1 / 2} \\
\frac{\partial f}{\partial e^{\prime}} & =-b_{0}^{-3 / 2}\left[\frac{1}{a^{\prime}} \frac{\partial r^{\prime}}{\partial e^{\prime}}\left(1-\frac{r}{a^{\prime}} c_{0}\right)-\frac{r}{a^{\prime}} \frac{\partial c}{\partial v^{\prime}} \frac{\partial v^{\prime}}{d e^{\prime}}\right] \\
\frac{\partial^{2} f}{\partial e^{\prime 2}} & =-b_{0}^{-3 / 2}\left[\left(\frac{1}{a^{\prime}} \frac{\partial r^{\prime}}{\partial e^{\prime}}\right)^{2}+\frac{1}{a^{\prime}} \frac{\partial^{2} r^{\prime}}{\partial e^{\prime 2}}\left(1-\frac{r}{a^{\prime}} c_{0}\right)\right. \\
& -\frac{r}{a^{\prime}} \frac{\partial c}{\partial v^{\prime}} \frac{\partial^{2} v^{\prime}}{\partial e^{\prime 2}}-2 \frac{r}{a^{\prime}} \frac{\partial c}{\partial v^{\prime}} \frac{\partial v^{\prime}}{\partial e^{\prime}}\left(\frac{1}{a^{\prime}} \frac{\partial r^{\prime}}{\partial e^{\prime}}\right) \\
\frac{\partial^{2} f}{\partial \eta^{\prime 2}} & \left.=b_{0}^{-3 / 2} \frac{r}{a^{\prime}} \frac{\partial^{2} c}{\partial \eta^{\prime 2}} \frac{\partial^{2} c}{\partial v^{\prime 2}}\left(\frac{\partial v^{\prime}}{\partial e^{\prime}}\right)^{2}\right]+3 b_{0}^{1 / 2}\left(\frac{\partial f}{\partial e^{\prime}}\right)^{2} \\
\frac{\partial^{2} f}{\partial \eta^{2}} & =b_{0}^{-3 / 2} \frac{r}{a^{\prime}} \frac{\partial^{2} c}{\partial \eta^{2}} \\
\frac{\partial^{4} f}{\partial \eta^{4}} & =9 b_{0}^{1 / 2}\left(\frac{\partial^{2} f}{\partial \eta^{2}}\right)^{2} \\
\frac{\partial^{3} f}{\partial e^{\prime} \partial \eta^{2}} & =b_{0}^{-3 / 2}\left[\frac{r}{a^{\prime}} \frac{\partial^{2} c}{\partial \eta^{2}}\left(\frac{1}{a^{\prime}} \frac{\partial r^{\prime}}{\partial e^{\prime}}\right)+\frac{r}{a^{\prime}} \frac{\partial^{3} c}{\partial v^{\prime} \partial \eta^{2}} \frac{\partial v^{\prime}}{\partial e^{\prime}}\right]
\end{aligned}
$$

$$
\begin{array}{r}
+3 b_{0}^{1 / 2} \frac{\partial f}{\partial e^{\prime}} \frac{\partial^{2} f}{\partial \eta^{2}} \\
\frac{\partial^{2} f}{\partial \eta^{\prime} \partial \eta}=b_{0}^{-3 / 2} \frac{r}{a^{\prime}} \frac{\partial^{2} c}{\partial \eta^{\prime} \partial \eta}
\end{array}
$$

where $c_{0}=\left.\cos S\right|_{e^{\prime}=\eta^{\prime}=\eta=0}$ and

$b_{0}=1+\frac{r^{2}}{a^{\prime 2}}-2 \frac{r}{a^{\prime}} c_{0}$.

For the indirect part, after straightforward calculations, we obtain

$$
\begin{aligned}
f_{0}^{1}= & -\frac{r}{a^{\prime}} c_{0} \\
\frac{\partial f^{1}}{\partial e^{\prime}=} & -\frac{r}{a^{\prime}} \frac{\partial c}{\partial v^{\prime}} \frac{\partial v^{\prime}}{\partial e^{\prime}}+2 \frac{r}{a^{\prime}} c_{0}\left(\frac{1}{a^{\prime}} \frac{\partial r^{\prime}}{\partial e^{\prime}}\right) \\
\frac{\partial^{2} f^{1}}{\partial e^{\prime 2}}= & -6 \frac{r}{a^{\prime}} c_{0}\left(\frac{1}{a^{\prime}} \frac{\partial r^{\prime}}{\partial e^{\prime}}\right)^{2}+2 \frac{r}{a^{\prime}} c_{0}\left(\frac{1}{a^{\prime}} \frac{\partial^{2} r^{\prime}}{\partial e^{\prime 2}}\right) \\
& +4 \frac{r}{a^{\prime}} \frac{\partial c}{\partial v^{\prime}} \frac{\partial v^{\prime}}{\partial e^{\prime}}\left(\frac{1}{a^{\prime}} \frac{\partial r^{\prime}}{\partial e^{\prime}}\right)-\frac{r}{a^{\prime}} \frac{\partial c}{\partial v^{\prime}} \frac{\partial^{2} v^{\prime}}{\partial e^{\prime 2}} \\
\frac{\partial^{2} f^{1}}{\partial \eta^{\prime 2}}= & -\frac{r}{a^{\prime}} \frac{\partial^{2} c}{\partial v^{\prime 2}}\left(\frac{\partial v^{\prime}}{\partial e^{\prime}}\right)^{2} \frac{\partial^{2} c}{\partial \eta^{\prime 2}} \\
\frac{\partial^{2} f^{1}}{\partial \eta^{2}}= & -\frac{r}{a^{\prime}} \frac{\partial^{2} c}{\partial \eta^{2}} \\
\frac{\partial^{4} f^{1}}{\partial \eta^{4}}= & 0 \\
\frac{\partial^{3} f^{1}}{\partial e^{\prime} \partial \eta^{2}}= & -\frac{r}{a^{\prime}} \frac{\partial^{3} c}{\partial v^{\prime} \partial \eta^{2}} \frac{\partial v^{\prime}}{\partial e^{\prime}}+2 \frac{r}{a^{\prime}} \frac{\partial^{2} c}{\partial \eta^{2}}\left(\frac{1}{a^{\prime}} \frac{\partial r^{\prime}}{\partial e^{\prime}}\right) \\
\frac{\partial^{2} f^{1}}{\partial \eta^{\prime} \partial \eta}= & -\frac{r}{a^{\prime}} \frac{\partial^{2} c}{\partial \eta^{\prime} \partial \eta} .
\end{aligned}
$$

Now, we introduce the functions

$$
\begin{aligned}
& W_{1}=\frac{r}{a} \cos (v-\sigma) \\
& W_{2}=\frac{r}{a} \sin (v-\sigma)
\end{aligned}
$$

which are the same as defined in FMS89 (see Eq. 18 in that paper). These definitions together with Eq. (7) lead, after some calculations, to the following expressions for the derivatives at $e^{\prime}=\eta^{\prime}=\eta=0$

$$
\begin{aligned}
\frac{r^{2}}{a^{\prime 2}}= & \alpha^{2}\left(W_{1}^{2}+W_{2}^{2}\right) \\
\frac{r}{a^{\prime}} c_{0}= & \alpha\left(W_{1} \cos p Q+W_{2} \sin p Q\right) \\
\frac{r}{a^{\prime}} \frac{\partial c}{\partial v^{\prime}}= & -\alpha\left(W_{1} \sin p Q-W_{2} \cos p Q\right) \\
\frac{r}{a^{\prime}} \frac{\partial^{2} c}{\partial \eta^{2}}= & -4 \alpha\left(W_{1} \sin \sigma_{z}+W_{2} \cos \sigma_{z}\right) \\
& \times\left(\sin p Q \cos \sigma_{z}+\cos p Q \sin \sigma_{z}\right)
\end{aligned}
$$




$$
\begin{aligned}
\frac{r}{a^{\prime}} \frac{\partial^{2} c}{\partial \eta^{\prime} \partial \eta}= & 4 \alpha\left(W_{1} \sin \sigma_{z}+W_{2} \cos \sigma_{z}\right) \\
& \times\left(\sin p Q \cos \sigma_{z}^{\prime}+\cos p Q \sin \sigma_{z}^{\prime}\right) \\
\frac{r}{a^{\prime}} \frac{\partial^{3} c}{\partial v^{\prime} \partial \eta^{2}}= & -4 \alpha\left(W_{1} \sin \sigma_{z}+W_{2} \cos \sigma_{z}\right) \\
& \times\left(\cos p Q \cos \sigma_{z}-\sin p Q \sin \sigma_{z}\right) \\
\frac{r}{a^{\prime}} \frac{\partial^{2} c}{\partial v^{\prime 2}}= & -\alpha\left(W_{1} \cos p Q+W_{2} \sin p Q\right)
\end{aligned}
$$

where $\alpha=a / a^{\prime}$ (recall that $a^{\prime}$ is a constant). From Eqs. (6) we also have

$$
\begin{aligned}
\frac{1}{a^{\prime}} \frac{\partial r^{\prime}}{\partial e^{\prime}} & =-\cos p Q \cos \sigma^{\prime}+\sin p Q \sin \sigma^{\prime} \\
\frac{\partial v^{\prime}}{\partial e^{\prime}} & =2\left(\cos p Q \sin \sigma^{\prime}+\sin p Q \cos \sigma^{\prime}\right) \\
\frac{1}{a^{\prime}} \frac{\partial^{2} r^{\prime}}{\partial e^{\prime 2}} & =1-\cos 2 p Q \cos 2 \sigma^{\prime}+\sin 2 p Q \sin 2 \sigma^{\prime} \\
\frac{\partial^{2} v^{\prime}}{\partial e^{\prime 2}} & =\frac{5}{2}\left(\cos 2 p Q \sin 2 \sigma^{\prime}+\sin 2 p Q \cos 2 \sigma^{\prime}\right)
\end{aligned}
$$

and the computation of the derivatives is almost completed.

It is worth noting that Eqs. (15) can be written in the form

$$
\begin{aligned}
& W_{1}=g_{2} \cos (p+q) Q-g_{3} \sin (p+q) Q \\
& W_{2}=g_{2} \sin (p+q) Q+g_{3} \cos (p+q) Q
\end{aligned}
$$

where $g_{2}, g_{3}$ are the functions

$$
\begin{aligned}
& g_{2}=\frac{r}{a} \cos (\varphi-\lambda) \\
& g_{3}=\frac{r}{a} \sin (\varphi-\lambda)
\end{aligned}
$$

with $\varphi=v+\varpi$. Thus, using the notation of FMS89 (see their Eqs. 11, 12, 14 and 37) we introduce the definitions

$$
\begin{aligned}
g_{1} & =W_{1}^{2}+W_{2}^{2} \\
& =g_{2}^{2}+g_{3}^{2} \\
g_{4} & =W_{1} \cos p Q+W_{2} \sin p Q \\
& =g_{2} \cos q Q-g_{3} \sin q Q \\
g_{5} & =W_{2} \cos p Q-W_{1} \sin p Q \\
& =g_{2} \sin q Q+g_{3} \cos q Q .
\end{aligned}
$$

Recall that $g_{4}$ differs by a factor $\alpha$ and $g_{5}$ differs by a factor $2 \alpha$ to their counterparts in FMS89. In addition, we define the functions

$$
\begin{aligned}
g_{6} & =W_{1} \cos p Q-W_{2} \sin p Q \\
& =g_{2} \cos (q+2 p) Q-g_{3} \sin (q+2 p) Q \\
g_{7} & =W_{2} \cos p Q+W_{1} \sin p Q \\
& =g_{2} \sin (q+2 p) Q+g_{3} \cos (q+2 p) Q .
\end{aligned}
$$

Finally, after some algebra, we obtain the following results for both the direct and indirect parts.

\subsection{Term of zero order}

It is

$\mathscr{R}_{0}=\left(f_{0}+f_{0}^{1}\right)$

where

$$
\begin{aligned}
& f_{0}=\left(1+\alpha^{2} g_{1}-2 \alpha g_{4}\right)^{-1 / 2} \\
& f_{0}^{1}=-\alpha g_{4}
\end{aligned}
$$

(compare with Eqs. 7 and 13 of FMS89).

\subsection{Terms of $\odot\left(e^{\prime}\right)$}

The term in $e^{\prime}$ is:

$\frac{\partial \mathscr{R}}{\partial e^{\prime}}=\left(f_{1}+f_{1}^{1}\right) \cos \sigma^{\prime}+\left(f_{2}+f_{2}^{1}\right) \sin \sigma^{\prime}$

where

$f_{1}=f_{0}^{3}\left[\left(1-\alpha g_{4}\right) \cos p Q+2 \alpha g_{5} \sin p Q\right]$

$f_{2}=-f_{0}^{3}\left[\left(1-\alpha g_{4}\right) \sin p Q-2 \alpha g_{5} \cos p Q\right]$

$f_{1}^{1}=-2 \alpha\left(g_{4} \cos p Q+g_{5} \sin p Q\right)$

$f_{2}^{1}=2 \alpha\left(g_{4} \sin p Q-g_{5} \cos p Q\right)$

(compare with Eq. 36 of FMS89). The term in $\eta^{2}$ is:

$$
\begin{aligned}
\frac{\partial^{2} \mathscr{R}}{\partial \eta^{2}}= & \left(f_{3}+f_{3}^{1}\right)+\left(f_{4}+f_{4}^{1}\right) \cos 2 \sigma_{z} \\
& +\left(f_{5}+f_{5}^{1}\right) \sin 2 \sigma_{z}
\end{aligned}
$$

where

$f_{3}=-f_{0}^{3} 2 \alpha g_{4}$

$f_{4}=f_{0}^{3} 2 \alpha g_{6}$

$f_{5}=-f_{0}^{3} 2 \alpha g_{7}$

$f_{3}^{1}=2 \alpha g_{4}$

$f_{4}^{1}=-2 \alpha g_{6}$

$f_{5}^{1}=2 \alpha g_{7}$.

\subsection{Higher order terms}

The term in $e^{\prime 2}$ is:

$$
\begin{aligned}
\frac{\partial^{2} \mathscr{R}}{\partial e^{\prime 2}}= & \left(f_{6}+f_{6}^{1}\right)+\left(f_{7}+f_{7}^{1}\right) \cos 2 \sigma^{\prime} \\
& +\left(f_{8}+f_{8}^{1}\right) \sin 2 \sigma^{\prime}
\end{aligned}
$$

where

$f_{6}=-f_{0}^{3}\left(\frac{3}{2}+\alpha g_{4}\right)+\frac{3}{2} f_{0}^{5}\left[\left(1-\alpha g_{4}\right)^{2}+\left(2 \alpha g_{5}\right)^{2}\right]$ $f_{7}=f_{0}^{3}\left[\left(\frac{1}{2}+\alpha g_{4}\right) \cos 2 p Q+\frac{1}{2} \alpha g_{5} \sin 2 p Q\right]$ 


$$
\begin{aligned}
+ & \frac{3}{2} f_{0}^{5}\left[\left(\left(1-\alpha g_{4}\right)^{2}-\left(2 \alpha g_{5}\right)^{2}\right) \cos 2 p Q\right. \\
& \left.+4\left(1-\alpha g_{4}\right) \alpha g_{5} \sin 2 p Q\right] \\
f_{8}= & -f_{0}^{3}\left[\left(\frac{1}{2}+\alpha g_{4}\right) \sin 2 p Q-\frac{1}{2} \alpha g_{5} \cos 2 p Q\right] \\
& -\frac{3}{2} f_{0}^{5}\left[\left(\left(1-\alpha g_{4}\right)^{2}-\left(2 \alpha g_{5}\right)^{2}\right) \sin 2 p Q\right. \\
& \left.\quad-4\left(1-\alpha g_{4}\right) \alpha g_{5} \cos 2 p Q\right] \\
f_{6}^{1}= & \alpha g_{4} \\
f_{7}^{1}= & -7 \alpha g_{4} \cos 2 p Q-\frac{13}{2} \alpha g_{5} \sin 2 p Q \\
f_{8}^{1}= & 7 \alpha g_{4} \sin 2 p Q-\frac{13}{2} \alpha g_{5} \cos 2 p Q
\end{aligned}
$$

(compare with Eqs. 40, 41, 42 and 43 of FMS89). The term in $\eta^{\prime 2}$ is almost the same as Eq. (27):

$$
\begin{aligned}
\frac{\partial^{2} \mathscr{R}}{\partial \eta^{\prime 2}}= & \left(f_{3}+f_{3}^{1}\right)+\left(f_{4}+f_{4}^{1}\right) \cos 2 \sigma_{z}^{\prime} \\
& +\left(f_{5}+f_{5}^{1}\right) \sin 2 \sigma_{z}^{\prime} .
\end{aligned}
$$

The term in $\eta^{4}$ has no indirect part:

$$
\begin{aligned}
\frac{\partial^{4} \mathscr{R}}{\partial \eta^{4}}= & f_{9}+f_{10} \cos 2 \sigma_{z}+f_{11} \sin 2 \sigma_{z} \\
& +f_{12} \cos 4 \sigma_{z}+f_{13} \sin 4 \sigma_{z}
\end{aligned}
$$

where

$$
\begin{aligned}
f_{9} & =18 f_{0}^{5} \alpha^{2}\left(g_{1}+2 g_{4}^{2}\right) \\
f_{10} & =-72 f_{0}^{5} \alpha^{2} g_{4} g_{6} \\
f_{11} & =72 f_{0}^{5} \alpha^{2} g_{4} g_{7} \\
f_{12} & =18 f_{0}^{5} \alpha^{2}\left(g_{6}^{2}-g_{7}^{2}\right) \\
f_{13} & =-36 f_{0}^{5} \alpha^{2} g_{6} g_{7} .
\end{aligned}
$$

And finaly, the mixed term in $e^{\prime} \eta^{2}$ is:

$$
\begin{aligned}
\frac{\partial^{3} \mathscr{R}}{\partial e^{\prime} \partial \eta^{2}}=[ & \left(f_{14}+f_{14}^{1}\right)+\left(f_{15}+f_{15}^{1}\right) \cos 2 \sigma_{z} \\
& \left.+\left(f_{16}+f_{16}^{1}\right) \sin 2 \sigma_{z}\right] \cos \sigma^{\prime} \\
+ & {\left[\left(f_{17}+f_{17}^{1}\right)+\left(f_{18}+f_{18}^{1}\right) \cos 2 \sigma_{z}\right.} \\
& \left.+\left(f_{19}+f_{19}^{1}\right) \sin 2 \sigma_{z}\right] \sin \sigma^{\prime}
\end{aligned}
$$

where

$$
\begin{aligned}
& f_{14}=f_{0}^{3} \alpha\left(3 W_{1}-g_{4} \cos p Q-g_{5} \sin p Q\right)-6 f_{0}^{2} f_{1} \alpha g_{4} \\
& f_{15}=-f_{0}^{3} \alpha\left(3 W_{1}-g_{6} \cos p Q+g_{7} \sin p Q\right)+6 f_{0}^{2} f_{1} \alpha g_{6} \\
& f_{16}=f_{0}^{3} \alpha\left(3 W_{2}-g_{6} \sin p Q-g_{7} \cos p Q\right)-6 f_{0}^{2} f_{1} \alpha g_{7} \\
& f_{17}=-f_{0}^{3} \alpha\left(3 W_{2}-g_{4} \sin p Q+g_{5} \cos p Q\right)-6 f_{0}^{2} f_{2} \alpha g_{4} \\
& f_{18}=-f_{0}^{3} \alpha\left(3 W_{2}+g_{6} \sin p Q+g_{7} \cos p Q\right)+6 f_{0}^{2} f_{2} \alpha g_{6} \\
& f_{19}=-f_{0}^{3} \alpha\left(3 W_{1}+g_{6} \cos p Q-g_{7} \sin p Q\right)-6 f_{0}^{2} f_{2} \alpha g_{7}
\end{aligned}
$$

$f_{14}^{1}=-2 f_{1}^{1}$

$f_{15}^{1}=-f_{19}^{1}=-4 \alpha\left(g_{6} \cos p Q-g_{7} \sin p Q\right)$

$f_{16}^{1}=f_{18}^{1}=4 \alpha\left(g_{6} \sin p Q+g_{7} \cos p Q\right)$

$f_{17}^{1}=2 f_{2}^{1}$.

All these terms are of $\mathscr{G}\left(e^{\prime 2}\right)$. There is also a mixed term in $\eta^{\prime} \eta$ which is of $\mathscr{Q}\left(e^{13 / 2}\right)$; it is:

$$
\begin{aligned}
\frac{\partial^{2} \mathscr{R}}{\partial \eta^{\prime} \partial \eta}= & {\left[\left(f_{20}+f_{20}^{1}\right) \cos \sigma_{z}^{\prime}+\left(f_{21}+f_{21}^{1}\right) \sin \sigma_{z}^{\prime}\right] \sin \sigma_{z} } \\
& +\left[\left(f_{22}+f_{22}^{1}\right) \cos \sigma_{z}^{\prime}+\left(f_{23}+f_{23}^{1}\right) \sin \sigma_{z}^{\prime}\right] \cos \sigma_{z}
\end{aligned}
$$

where

$$
\begin{aligned}
& f_{20}=f_{0}^{3} 4 \alpha W_{1} \sin p Q=f_{0}^{3} 2 \alpha\left(g_{7}-g_{5}\right) \\
& f_{21}=f_{0}^{3} 4 \alpha W_{1} \cos p Q=f_{0}^{3} 2 \alpha\left(g_{4}+g_{6}\right) \\
& f_{22}=f_{0}^{3} 4 \alpha W_{2} \sin p Q=f_{0}^{3} 2 \alpha\left(g_{4}-g_{6}\right) \\
& f_{23}=f_{0}^{3} 4 \alpha W_{2} \cos p Q=f_{0}^{3} 2 \alpha\left(g_{7}+g_{5}\right) \\
& f_{20}^{1}=-4 \alpha W_{1} \sin p Q=-2 \alpha\left(g_{7}-g_{5}\right) \\
& f_{21}^{1}=-4 \alpha W_{1} \cos p Q=-2 \alpha\left(g_{4}+g_{6}\right) \\
& f_{22}^{1}=-4 \alpha W_{2} \sin p Q=-2 \alpha\left(g_{4}-g_{6}\right) \\
& f_{23}^{1}=-4 \alpha W_{2} \cos p Q .=-2 \alpha\left(g_{7}+g_{5}\right) .
\end{aligned}
$$

With these calculations we have completed the first part of the expansion.

\section{Expansion in $k, h$ and $\alpha$}

We know that, in the resonance, the critical argument $\sigma$ librates around a certain value $\sigma_{0}$, and both the eccentricity and the semimajor axis librate around $e_{0}$ and $a_{0}$ respectively. So we could make an expansion of the disturbing function around these points. Since $e$ and $\sigma$ are coupled through the D'Alembert rule, any term with the argument $n \sigma$ is proportional to $e^{n}$. Thus any expansion around a point $e_{0} \neq 0$ is singular at $e=0$. In order to avoid this non-analyticity at the origin it is useful to replace the pair of variables $e, \sigma$ by the regular variables

$$
\begin{aligned}
& k=e \cos \sigma \\
& h=e \sin \sigma
\end{aligned}
$$

and to expand $R$ around the point $\left(k_{0}, h_{0}\right)$.

It is worth noting that the expressions obtained in Sect. 3 depend on $k, h$ and $\alpha$ only through the coefficients $f_{i}$ and $f_{i}^{1}$, and more precisely, they depend on $k, h$ only through the functions $W_{1}$ and $W_{2}$ (or equivalently, the functions $g_{2}$ and $g_{3}$ ). Thus, the second part of the expansion consists in expanding each of these coefficients in powers of $\left(h-h_{0}\right),\left(k-k_{0}\right)$ and $\left(\alpha-\alpha_{0}\right)$.

We assume that all $\Delta k, \Delta h$ and $\Delta \alpha$ are of order $\mathscr{O}\left(e^{\prime}\right)$ (i.e., $\Delta e \simeq \mathscr{O}\left(e^{\prime}\right)$ and $\left.\Delta \alpha \simeq 0.05\right)$. So, we expand the term $\mathscr{R}_{0}$ up to second the order in $e^{\prime}$ :

$\mathscr{R}_{0}=\mathscr{R}_{00}+\frac{\partial \cdot \mathscr{R}_{0}}{\partial k}\left(k-k_{0}\right)+\frac{\partial \mathscr{R}_{0}}{\partial h}\left(h-h_{0}\right)$ 


$$
\begin{aligned}
& +\frac{\partial \mathscr{R}_{0}}{\partial \alpha}\left(\alpha-\alpha_{0}\right)+\frac{1}{2} \frac{\partial^{2} \mathscr{R}_{0}}{\partial k^{2}}\left(k-k_{0}\right)^{2} \\
& +\frac{1}{2} \frac{\partial^{2} \mathscr{R}_{0}}{\partial h^{2}}\left(h-h_{0}\right)^{2}+\frac{1}{2} \frac{\partial^{2} \cdot \mathscr{R}_{0}}{\partial \alpha^{2}}\left(\alpha-\alpha_{0}\right)^{2} \\
& +\frac{\partial^{2} \mathscr{R}_{0}}{\partial k \partial h}\left(k-k_{0}\right)\left(h-h_{0}\right)+\frac{\partial^{2} \cdot \mathscr{R}_{0}}{\partial h \partial \alpha}\left(h-h_{0}\right)\left(\alpha-\alpha_{0}\right) \\
& +\frac{\partial^{2} \mathscr{R}_{0}}{\partial k \partial \alpha}\left(k-k_{0}\right)\left(\alpha-\alpha_{0}\right) .
\end{aligned}
$$

The terms of $\mathscr{O}\left(e^{\prime}\right)$ are expanded only up to the first order in $e^{\prime}$ obtaining

$$
\begin{aligned}
\frac{\partial \mathscr{R}}{\partial e^{\prime}}= & \left(\frac{\partial \mathscr{R}}{\partial e^{\prime}}\right)_{0}+\frac{\partial}{\partial k} \frac{\partial \mathscr{R}}{\partial e^{\prime}}\left(k-k_{0}\right) \\
& +\frac{\partial}{\partial h} \frac{\partial \mathscr{R}}{\partial e^{\prime}}\left(h-h_{0}\right)+\frac{\partial}{\partial \alpha} \frac{\partial \mathscr{R}}{\partial e^{\prime}}\left(\alpha-\alpha_{0}\right) \\
\frac{\partial^{2} \mathscr{R}}{\partial \eta^{2}}= & \left(\frac{\partial^{2} \mathscr{R}}{\partial \eta^{2}}\right)_{0}+\frac{\partial}{\partial k} \frac{\partial^{2} \mathscr{R}}{\partial \eta^{2}}\left(k-k_{0}\right) \\
& +\frac{\partial}{\partial h} \frac{\partial^{2} \mathscr{R}}{\partial \eta^{2}}\left(h-h_{0}\right)+\frac{\partial}{\partial \alpha} \frac{\partial^{2} \mathscr{R}}{\partial \eta^{2}}\left(\alpha-\alpha_{0}\right) .
\end{aligned}
$$

The remaining terms are just evaluated at $k_{0}, h_{0}$ and $\alpha_{0}$.

The calculation of these derivatives is almost simple. We show below some of the most cumbersome derivations of the direct part. Denoting by $X, Y$ any of the variables $k, h$ or $\alpha$, we have

$$
\begin{aligned}
\frac{\partial f_{0}}{\partial X}= & -\frac{1}{2} f_{0}^{3}\left[\frac{\partial\left(\alpha^{2} g_{1}\right)}{\partial X}-2 \frac{\partial\left(\alpha g_{4}\right)}{\partial X}\right] \\
\frac{\partial^{2} f_{0}}{\partial X \partial Y}= & 3 f_{0}^{-1} \frac{\partial f_{0}}{\partial X} \frac{\partial f_{0}}{\partial Y} \\
& -\frac{1}{2} f_{0}^{3}\left[\frac{\partial^{2}\left(\alpha^{2} g_{1}\right)}{\partial X \partial Y}-2 \frac{\partial^{2}\left(\alpha g_{4}\right)}{\partial X \partial Y}\right] \\
\frac{\partial f_{1}}{\partial X}= & 3 f_{0}^{-1} \frac{\partial f_{0}}{\partial X} f_{1} \\
& +f_{0}^{3}\left[-\frac{\partial\left(\alpha g_{4}\right)}{\partial X} \cos p Q+2 \frac{\partial\left(\alpha g_{5}\right)}{\partial X} \sin p Q\right] \\
\frac{\partial f_{3}}{\partial X}= & -6 f_{0}^{2} \frac{\partial f_{0}}{\partial X} \alpha g_{4}-2 f_{0}^{3} \frac{\partial\left(\alpha g_{4}\right)}{\partial X} .
\end{aligned}
$$

The explicit form of these coefficients can be found in Roig (1997).

\section{The functions $W_{1}$ and $W_{2}$}

The evaluation of the functions $W_{1}$ and $W_{2}$ as well as of their derivatives at the point $k_{0}, h_{0}$ can be done in two different ways as already described in FMS89. We can use the relations (18) together with the classical elliptic expansions of the functions $g_{2}$ and $g_{3}$ (see Sect. 7 of FMS89), which leads to the so-called high-eccentricity (HE) asymmetric expansion, valid only for $e_{0}<0.6627$, i.e., inside the radius of convergence of the cited expansions of $g_{2}$ and $g_{3}$. Alternatively, we can write the functions $W_{1}$ and $W_{2}$ in the form

$$
\begin{aligned}
& W_{1}=\cos (u-\sigma)-k+h T \\
& W_{2}=\sin (u-\sigma)+h+k T
\end{aligned}
$$

where

$T=\frac{1}{e}\left(\sqrt{1-e^{2}}-1\right) \sin u$

and we can calculate the derivatives with the use of closed formulas which are not singular at $e=0$ (see Sects. 3.1, 3.2 and 3.3 of FMS89 and pay attention to some obvious misprints in Eqs. 28 and 32, and in the formula after Eq. 22). This later method leads to the so-called very-high-eccentricity (VHE) asymmetric expansion, valid for any eccentricity $e_{0}$.

It is worth noting that in Eq. (46), $W_{1}$ and $W_{2}$ depend on the eccentric anomaly $u$. Thus, we must use the relation

$\ell=\sigma+(p+q) Q$

and to solve Kepler's equation in order to obtain the dependence of $W_{1}, W_{2}$ on $Q$.

\section{Final expression and average}

Now, we can write the final expression of the expansion. Denoting $\mathscr{R}_{i}=f_{i}+f_{i}^{1}$ and $\mathscr{R}_{i X}=\frac{\partial \mathscr{R}}{\partial X}$, we have

$$
\begin{aligned}
& R=\frac{\mathrm{G} m^{\prime}}{a^{\prime}}\left[\mathscr{R}_{0}+\mathscr{R}_{0 h}\left(h-h_{0}\right)+\mathscr{R}_{0 k}\left(k-k_{0}\right)\right. \\
& +\mathscr{R}_{0 \alpha}\left(\alpha-\alpha_{0}\right)+\left[\mathscr{R}_{1} \cos \sigma^{\prime}+\mathscr{R}_{2} \sin \sigma^{\prime}\right] e^{\prime} \\
& +\left[\mathscr{R}_{3}+\mathscr{R}_{4} \cos 2 \sigma_{z}+\mathscr{R}_{5} \sin 2 \sigma_{z}\right] \frac{1}{2} \eta^{2} \\
& +\mathscr{R}_{0 h h} \frac{1}{2}\left(h-h_{0}\right)^{2}+\mathscr{R}_{0 k k} \frac{1}{2}\left(k-k_{0}\right)^{2} \\
& +\mathscr{R}_{0 \alpha \alpha} \frac{1}{2}\left(\alpha-\alpha_{0}\right)^{2}+\mathscr{R}_{0 h k}\left(h-h_{0}\right)\left(k-k_{0}\right) \\
& +\mathscr{R}_{0 h \alpha}\left(h-h_{0}\right)\left(\alpha-\alpha_{0}\right)+\mathscr{R}_{0 k \alpha}\left(k-k_{0}\right)\left(\alpha-\alpha_{0}\right) \\
& +\left[\mathscr{R}_{1 h} \cos \sigma^{\prime}+\mathscr{R}_{2 h} \sin \sigma^{\prime}\right] e^{\prime}\left(h-h_{0}\right) \\
& +\left[\mathscr{R}_{1 k} \cos \sigma^{\prime}+\mathscr{R}_{2 k} \sin \sigma^{\prime}\right] e^{\prime}\left(k-k_{0}\right) \\
& +\left[\mathscr{R}_{1 \alpha} \cos \sigma^{\prime}+\mathscr{R}_{2 \alpha} \sin \sigma^{\prime}\right] e^{\prime}\left(\alpha-\alpha_{0}\right) \\
& +\left[\mathscr{R}_{3 h}+\mathscr{R}_{4 h} \cos 2 \sigma_{z}+\mathscr{R}_{5 h} \sin 2 \sigma_{z}\right] \frac{1}{2} \eta^{2}\left(h-h_{0}\right) \\
& +\left[\mathscr{R}_{3 k}+\mathscr{R}_{4 k} \cos 2 \sigma_{z}+\mathscr{R}_{5 k} \sin 2 \sigma_{z}\right] \frac{1}{2} \eta^{2}\left(k-k_{0}\right) \\
& +\left[\mathscr{R}_{3 \alpha}+\mathscr{R}_{4 \alpha} \cos 2 \sigma_{z}+\mathscr{R}_{5 \alpha} \sin 2 \sigma_{z}\right] \frac{1}{2} \eta^{2}\left(\alpha-\alpha_{0}\right) \\
& +\left[\mathscr{R}_{6}+\mathscr{R}_{7} \cos 2 \sigma^{\prime}+\mathscr{R}_{8} \sin 2 \sigma^{\prime}\right] \frac{1}{2} e^{\prime 2} \\
& +\left[\mathscr{R}_{3}+\mathscr{R}_{4} \cos 2 \sigma_{z}^{\prime}+\mathscr{R}_{5} \sin 2 \sigma_{z}^{\prime}\right] \frac{1}{2} \eta^{\prime 2} \\
& +\left[\mathscr{R}_{9}+\mathscr{R}_{10} \cos 2 \sigma_{z}+\mathscr{R}_{11} \sin 2 \sigma_{z}\right] \frac{1}{24} \eta^{4} \\
& +\left[\mathscr{R}_{12} \cos 4 \sigma_{z}+\mathscr{R}_{13} \sin 4 \sigma_{z}\right] \frac{1}{24} \eta^{4} \\
& +\left[\mathscr{R}_{14}+\mathscr{R}_{15} \cos 2 \sigma_{z}+\mathscr{R}_{16} \sin 2 \sigma_{z}\right] \cos \sigma^{\prime} \frac{1}{2} \eta^{2} e^{\prime} \\
& +\left[\mathscr{R}_{17}+\mathscr{R}_{18} \cos 2 \sigma_{z}+\mathscr{R}_{19} \sin 2 \sigma_{z}\right] \sin \sigma^{\prime} \frac{1}{2} \eta^{2} e^{\prime}
\end{aligned}
$$




$$
\begin{aligned}
& +\left[\mathscr{R}_{20} \cos \sigma_{z}^{\prime}+\mathscr{R}_{21} \sin \sigma_{z}^{\prime}\right] \eta^{\prime} \eta \sin \sigma_{z} \\
& \left.+\left[\mathscr{R}_{22} \cos \sigma_{z}^{\prime}+\mathscr{R}_{23} \sin \sigma_{z}^{\prime}\right] \eta^{\prime} \eta \cos \sigma_{z}\right] .
\end{aligned}
$$

Since each of the coefficients $\mathscr{R}_{i}$ depends on the fast variable $Q$, this expansion can be averaged by solving numerically the integral

$R^{*}=\frac{1}{2 \pi} \int_{0}^{2 \pi} R[u(Q), Q] d Q$.

The calculation of this integral requires the solution of Kepler's equation as an intermediate step (see Sect. 5). It is easy to show that this step can be avoided by performing the average directly over the eccentric anomaly (cf. Moons, 1993). From Eq. (48) we write

$Q=\frac{\left(u-e_{0} \sin u\right)-\sigma_{0}}{(p+q)}$

where $e_{0}, \sigma_{0}$ are the values at the center of the expansion; then Eq. (50) can be replaced by

$R^{*}=\frac{1}{2 \pi(p+q)} \int_{0}^{2 \pi(p+q)}\left(1-e_{0} \cos u\right) R[u, Q(u)] d u$

\subsection{Parity rules}

There are some special cases in which the coefficients $\mathscr{R}_{i}$ have a well defined parity with respect to the variable $Q$ (or $u$ ). This property can be used to predict some zero averages (for odd coefficients) and reduce to half the interval of integration in the calculation of even coefficients.

The case of particular interest is the one in which we use a center of expansion on the $k$-axis, i.e. $h_{0}=0$. Such an expansion is useful to study first-order resonances $(q=1)$, since, in this case, libration occurs around $\sigma_{0}=0$. Then, we can see from Eq. (51) that the variable $Q$ is an odd function of $u$, so the parity of any function with respect to $Q$ is the same as with respect to $u$. This property can be used together with the parities of the functions $W_{i}$ (and their derivatives) with respect to $u$, shown in Table 1, to predict the parity of each coefficient.

With these rules, the coefficient $f_{0}$ as well as any power of it are always even functions of $u$. Consequently, for a first-order resonance the averaged expansion is reduced to the following expression:

$$
\begin{aligned}
R^{*}= & \frac{\mathrm{G} m^{\prime}}{a^{\prime}}\left[\mathscr{R}_{0}^{*}+\mathscr{R}_{0 k}^{*}\left(k-k_{0}\right)+\mathscr{R}_{0 \alpha}^{*}\left(\alpha-\alpha_{0}\right)\right. \\
& +\mathscr{R}_{1}^{*} e^{\prime} \cos \sigma^{\prime}+\left[\mathscr{R}_{3}^{*}+\mathscr{R}_{4}^{*} \cos 2 \sigma_{z}\right] \frac{1}{2} \eta^{2} \\
& +\mathscr{R}_{0 h h}^{*} \frac{1}{2} h^{2}+\mathscr{R}_{0 k k}^{*} \frac{1}{2}\left(k-k_{0}\right)^{2} \\
& +\mathscr{R}_{0 \alpha \alpha}^{*} \frac{1}{2}\left(\alpha-\alpha_{0}\right)^{2}+\mathscr{R}_{0 k \alpha}^{*}\left(k-k_{0}\right)\left(\alpha-\alpha_{0}\right) \\
& +\mathscr{R}_{2 h}^{*} e^{\prime} \sin \sigma^{\prime} h+\mathscr{R}_{1 k}^{*} e^{\prime} \cos \sigma^{\prime}\left(k-k_{0}\right)
\end{aligned}
$$

$$
\begin{aligned}
& +\mathscr{R}_{1 \alpha}^{*} e^{\prime} \cos \sigma^{\prime}\left(\alpha-\alpha_{0}\right)+\mathscr{R}_{5 h}^{*} \frac{1}{2} \eta^{2} \sin 2 \sigma_{z} h \\
& +\left[\mathscr{R}_{3 k}^{*}+\mathscr{R}_{4 k}^{*} \cos 2 \sigma_{z}\right] \frac{1}{2} \eta^{2}\left(k-k_{0}\right) \\
& +\left[\mathscr{R}_{3 \alpha}^{*}+\mathscr{R}_{4 \alpha}^{*} \cos 2 \sigma_{z}\right] \frac{1}{2} \eta^{2}\left(\alpha-\alpha_{0}\right) \\
& +\left[\mathscr{R}_{6}^{*}+. \mathscr{R}_{7}^{*} \cos 2 \sigma^{\prime}\right] \frac{1}{2} e^{\prime 2} \\
& +\left[\mathscr{R}_{3}^{*}+\mathscr{R}_{4}^{*} \cos 2 \sigma_{z}^{\prime}\right] \frac{1}{2} \eta^{\prime 2} \\
& +\left[\mathscr{R}_{9}^{*}+\mathscr{R}_{10}^{*} \cos 2 \sigma_{z}+\mathscr{R}_{12}^{*} \cos 4 \sigma_{z}\right] \frac{1}{24} \eta^{4} \\
& +\left[\mathscr{R}_{14}^{*}+\mathscr{R}_{15}^{*} \cos 2 \sigma_{z}\right] \frac{1}{2} \eta^{2} e^{\prime} \cos \sigma^{\prime} \\
& +\mathscr{R}_{19}^{*} \frac{1}{2} \eta^{2} \sin 2 \sigma_{z} e^{\prime} \sin \sigma^{\prime}+\mathscr{R}_{21}^{*} \eta \sin \sigma_{z} \eta^{\prime} \sin \sigma_{z}^{\prime} \\
& \left.+\mathscr{R}_{22}^{*} \eta \cos \sigma_{z} \eta^{\prime} \cos \sigma_{z}^{\prime}\right]
\end{aligned}
$$

where $\mathscr{R}_{i}^{*}=\frac{1}{\pi(p+q)} \int_{0}^{\pi(p+q)}\left(1-e_{0} \cos u\right) \mathscr{R}_{i} d u$.

In the general case, when the center of the expansion is at a point with $\sigma_{0} \neq n \pi / 2$ ( $n$ integer) the functions $W_{1}, W_{2}$ as well as the variable $Q$ do not have well-defined parities with respect to $u$. Thus, the full expression Eq. (49) shall be considered.

\section{The oblateness of the central body}

In resonant satellite problems, we must take into account the part of the disturbing function arising from the oblateness of the primary body. Using the classical Legendre expansion, we can write this oblateness potential in a first approximation as

$R^{J}=-\frac{\mathrm{G} M J_{2} b^{2}}{a^{3}}\left(\frac{r}{a}\right)^{-3} P_{2}(\sin \phi)+\cdots$

where $M, b$ and $J_{2}$ are the mass, the equatorial radius and the ellipticity factor of the potential of the central body; $r, \phi$ and $a$ are the planetocentric distance, the latitude over the equator and the semimajor axis of the disturbed body. $P_{2}$ is the Legendre polynomial

$P_{2}(x)=\frac{3}{2} x^{2}-\frac{1}{2}$

If the equator of the primary is the reference plane for definition of inclinations, the following relation holds:

$\sin \phi=\sin I \sin (v+\omega)$

which in terms of Eqs. (1) and (4) can be written as

$\sin \phi=2 \eta \sqrt{1-\eta^{2}} \sin \left((v-\sigma)+\sigma_{z}\right)$.

We now substitute Eqs. (55) and (57) in Eq. (54) and obtain

$$
\begin{aligned}
R^{J}=- & \frac{\mathrm{G} M J_{2} b^{2}}{a^{\prime 3}}\left[\mathscr{R}_{0}^{J}+\frac{1}{2} \eta^{2}\left(1-\eta^{2}\right)\right. \\
& \left.\times\left(\mathscr{R}_{1}^{J}+\mathscr{R}_{2}^{J} \cos 2 \sigma_{z}+\mathscr{R}_{3}^{J} \sin 2 \sigma_{z}\right)\right]
\end{aligned}
$$


Table 1. Parities with respect to $u$ for the case $q=1$.

Even

$$
W_{1}, W_{1 k}, W_{2 h}, W_{1 h h}, W_{1 k k}, W_{2 h k}
$$

Odd

$$
W_{2}, W_{1 h}, W_{2 k}, W_{2 h h}, W_{2 k k}, W_{1 h k}
$$

where $a^{\prime}$ is the semimajor axis of the disturbing body. Taking into account Eqs. (15) and (20), we have

$\mathscr{R}_{0}^{J}=-\frac{1}{2}\left(\alpha g_{1}^{1 / 2}\right)^{-3}$

(compare with Eq. 72 of FMS89). In addition, we introduce the definitions

$g_{8}=W_{1}^{2}-W_{2}^{2}$

$g_{9}=2 W_{1} W_{2}$

and we can write

$\mathscr{R}_{1}^{J}=-12 \cdot \mathscr{R}_{0}^{J}$

$\mathscr{R}_{2}^{J}=12 \mathscr{R}_{0}^{J} g_{1}^{-1} g_{8}$

$\mathscr{R}_{3}^{J}=-12 \cdot \mathscr{R}_{0}^{J} g_{1}^{-1} g_{9}$.

Now, we expand each term $\mathscr{R}_{i}^{J}$ around $k_{0}, h_{0}$ and $\alpha_{0}$ as in Sect. 4. The calculation of the derivatives is simple and the final expression reads

$$
\begin{aligned}
R^{J}=- & \frac{\mathrm{G} M J_{2} b^{2}}{a^{\prime 3}}\left[\mathscr{R}_{0}^{J}+\mathscr{R}_{0 h}^{J}\left(h-h_{0}\right)+\mathscr{R}_{0 k}^{J}\left(k-k_{0}\right)\right. \\
& +\mathscr{R}_{0 \alpha}^{J}\left(\alpha-\alpha_{0}\right)+\frac{1}{2} \mathscr{R}_{0 h h}^{J}\left(h-h_{0}\right)^{2} \\
& +\frac{1}{2} \mathscr{R}_{0 k k}^{J}\left(k-k_{0}\right)^{2}+\frac{1}{2} \cdot \mathscr{R}_{0 \alpha \alpha}^{J}\left(\alpha-\alpha_{0}\right)^{2} \\
& +\mathscr{R}_{0 h k}^{J}\left(h-h_{0}\right)\left(k-k_{0}\right)+\mathscr{R}_{0 h \alpha}^{J}\left(h-h_{0}\right)\left(\alpha-\alpha_{0}\right) \\
& +\mathscr{R}_{0 k \alpha}^{J}\left(k-k_{0}\right)\left(\alpha-\alpha_{0}\right)+\frac{1}{2} \eta^{2}\left(1-\eta^{2}\right) \\
& \times\left(\mathscr{R}_{1}^{J}+\mathscr{R}_{2}^{J} \cos 2 \sigma_{z}+\mathscr{R}_{3}^{J} \sin 2 \sigma_{z}\right) \\
& +\frac{1}{2} \eta^{2}\left(h-h_{0}\right) \\
& \times\left(\mathscr{R}_{1 h}^{J}+\mathscr{R}_{2 h}^{J} \cos 2 \sigma_{z}+\mathscr{R}_{3 h}^{J} \sin 2 \sigma_{z}\right) \\
+ & \frac{1}{2} \eta^{2}\left(k-k_{0}\right) \\
& \times\left(\mathscr{R}_{1 k}^{J}+\mathscr{R}_{2 k}^{J} \cos 2 \sigma_{z}+\mathscr{R}_{3 k}^{J} \sin 2 \sigma_{z}\right) \\
+ & \frac{1}{2} \eta^{2}\left(\alpha-\alpha_{0}\right) \\
& \left.\times\left(\mathscr{R}_{1 \alpha}^{J}+\mathscr{R}_{2 \alpha}^{J} \cos 2 \sigma_{z}+\mathscr{R}_{3 \alpha}^{J} \sin 2 \sigma_{z}\right)\right] .
\end{aligned}
$$

The average is computed in the same way as already described in Sect. 6, and parity rules apply when $h_{0}=0$.

\section{Comparisons}

In order to test the precision of our asymmetric expansion, we made several comparisons with the exact averaged resonant disturbing function, computed numerically using the formulas of
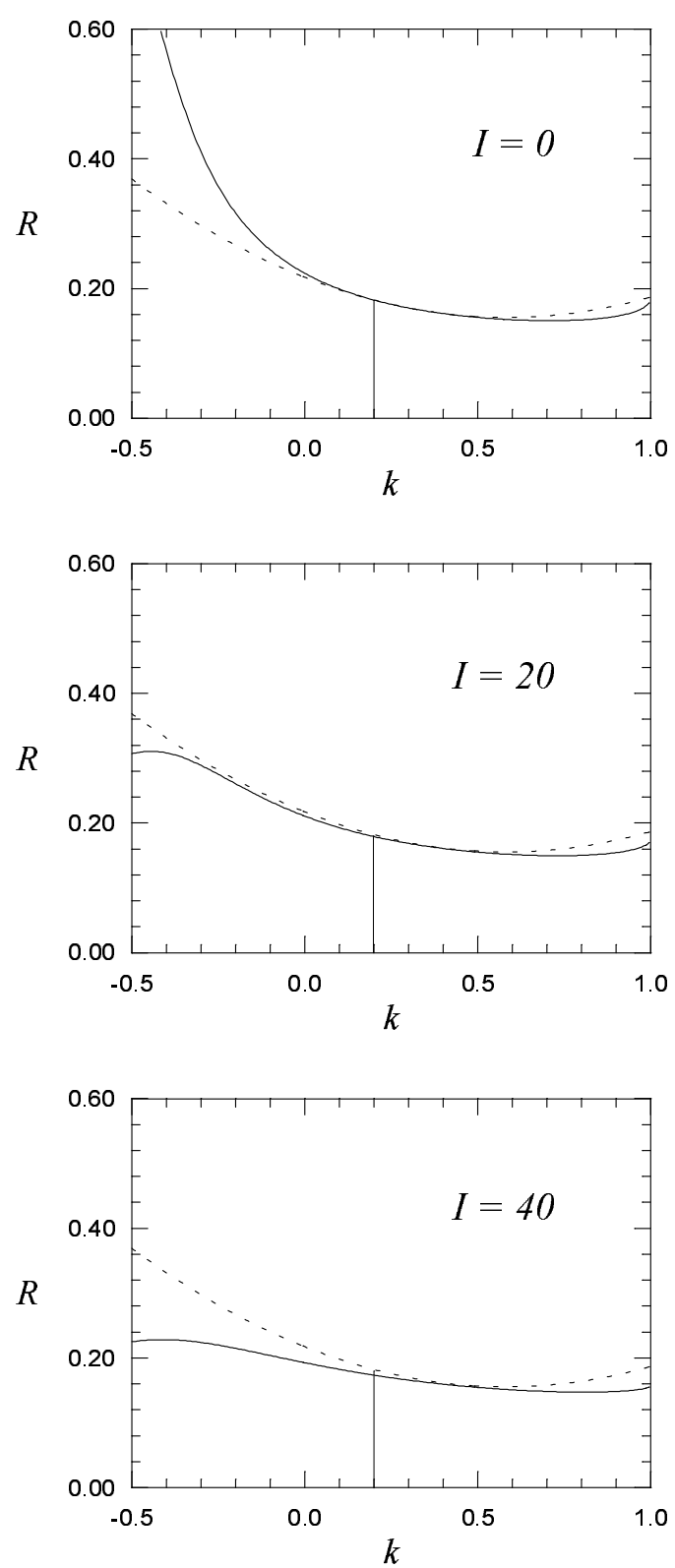

Fig. 1. Comparison at $\sigma=0$ between the exact averaged disturbing function (continous line) and the asymmetric expansion (dotted line), expanded around the point $k_{0}=0.2, h_{0}=0$ and $a_{0}=3.275 \mathrm{AU}(2 / 1$ resonance) for different values of $I$, considering $\sigma_{z}=0$. In all cases we take $a^{\prime}=5.2 \mathrm{AU}, e^{\prime}=0.05, I^{\prime}=1.2^{\circ}$, and $\sigma^{\prime}=\sigma_{z}^{\prime}=0$. The expansion is almost the same in all cases. The agreement at $I=20^{\circ}$ is better than at $I=0^{\circ}$ due to the fact that the planar disturbing function has a singularity at $k \simeq-0.5$.

Moons (1993). These comparisons were made for the $2 / 1$ resonance and in all cases we considered

$a^{\prime}=5.2 \mathrm{AU}$

$e^{\prime}=0.05$

$I^{\prime}=1.2^{\circ}$

$\sigma^{\prime}=\sigma_{z}^{\prime}=0$ 


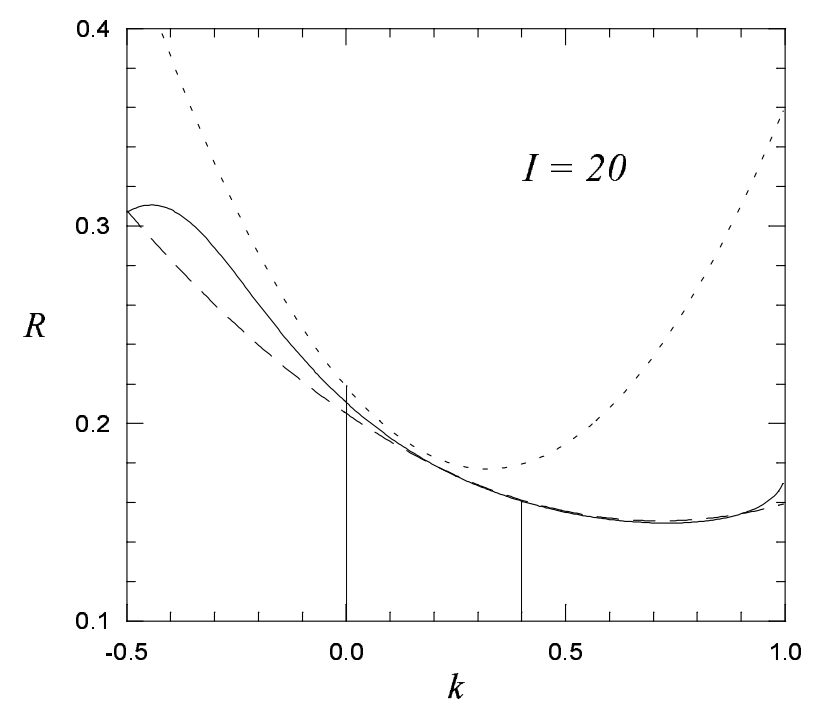

Fig. 2. Comparison at $\sigma=0$ between the exact disturbing function (continuous line) and two expansions around the points $k_{0}=h_{0}=0$ (dotted line) and $k_{0}=0.4, h_{0}=0$ (dashed line), with $a_{0}=3.275$ $\mathrm{AU}$ in both cases. The comparison is made for $I=20^{\circ}$ and $\sigma_{z}=0$. The variables of the disturbing body have the same values as in Fig. 1 . Note that at $k=0$ the difference between each expansion and the exact function is almost the same. However, the second expansion gives better approximation for other values of $k$ (see comment in the text).

as fixed parameters. The coefficients of the expansion were evaluated using the closed formulas as in the VHE expansion. The average was computed using a fourty points Gauss quadrature.

First, it is known that the planar asymmetric expansion becomes singular when the path of integration over $Q$ includes the point of collision of the bodies. The set of points in the $k_{0}, h_{0}$ plane where this singularity occurs is the so-called collision curve (see Ferraz-Mello et al., 1993). The non-planar asymmetric expansion presented here is the sum of the planar expansion plus terms related with the inclinations; so, this spatial expansion has embedded the presence of the collision curve of the planar problem. If we choose the center of expansion $\left(k_{0}, h_{0}\right)$ close to the collision curve, the spatial expansion will try to reproduce a false singularity, leading to a very bad representation of the disturbing function, even if a collision may occur only at the mutual node of the orbits.

However, when the center $\left(k_{0}, h_{0}\right)$ is far away from the collision curve, the expansion can be used to obtain a very good representation of the non-planar disturbing function. This representation can be even better than that of the planar disturbing function (FMS89), due to the fact that, in general, the non-planar function does not have a collision curve. We can see in Fig. 1 that, for a given center of expansion $k_{0}, h_{0}$, there seems to be a certain inclination for which the expansion works the best.

It is worth noting that the best agreement between the asymmetric expansion and the exact disturbing function at a given point in the plain $k, h$ is, sometimes, obtained using a center of expansion $k_{0}, h_{0}$ other than that point. This does not only depend on the values of $k_{0}, h_{0}$, but also on the values of $I, e^{\prime}$ and $I^{\prime}$ at which the expansion is evaluated. For example, we see in Fig. 2 that, around the origin $(k=0)$, the asymmetric expansion (for which $k_{0}=0.4$ ) gives an approximation as good as the Laplacian expansion (for which $k_{0}=0$ ). However, the asymmetric one gives better approximation for other values of $k$, so it can be applyed to problems in which the center of libration is at $k=0.4$ as well as at $k=0$. Note that, in this figure, neither the asymmetric expansion nor the Laplacian one coincide with the exact function when $k=k_{0}$. The difference with the exact function at this point is because both expansions are made around $e^{\prime}=I^{\prime}=I=0$, but they are being evaluated at $e^{\prime}=0.05, I^{\prime}=1.2^{\circ}$ and $I=20^{\circ}$. The difference is greater at $k_{0}=0$ because we are nearer the collision curve and the expansion becomes worse.

We also perfomed some comparisons for different values of $\sigma$. To do this, we used an asymmetric expansion around $a_{0}=$ 3.275 $\mathrm{AU}, h_{0}=0$ and a given $k_{0}$ between 0 and 0.8 . Then we fixed $a, I$ and $\sigma_{z}$ and calculated the relative difference between this expansion and the exact function at each point of a grid in the plane $e, \sigma$. The results were plotted as level curves in that plane. Some of these comparisons, for three different values of the inclination $\left(I=0^{\circ}, I=15^{\circ}, I=30^{\circ}\right)$ are shown in Fig. 3, using centers of expansion around $k_{0}=0.2$ and around $k_{0}=0.6$. In all these examples we fixed $a=3.275 \mathrm{AU}$ and $\sigma_{z}=0$.

In those figures the brightest level represents the region where the relative difference is below $1 \%$. The center of expansion is marked with a cross. We see that for inclinations $\sim 30^{\circ}$ (in the chosen limit for the truncation of the expansion), the approximation becomes bad, and can be better in other region than around the center of expansion. For smaller inclinations we obtained a good agreement even for amplitudes of libration of about $60^{\circ}$ or $90^{\circ}$. It is worth noting that the evaluation of the averaged asymmetric expansion in a grid of $100 \times 200$ points in the plane $e, \sigma$ is about ten times faster than the numerical evaluation of the exact averaged disturbing function in the same grid.

Although the asymmetric expansion gives a good approximation of the exact function in a wide region of the phase space, it is worth stressing the fact that the most important feature for the dynamics is to be able to reproduce with the expansion the location of the equilibrium solutions of the system (pericentric branch, corotation solutions, etc.). For example, we can see in Fig. 1 that for $I=20^{\circ}$ the location of the minimum of the expansion does not coincide with the minimum of the exact function. As this minimum is associated with the location of the Kozai resonance, we will not obtain a good representation of the dynamics for $e>0.5$ with this expansion. It is obvious that, in this case, we have to change the center of expansion and take a value of $k_{0}$ nearer to the minimum of the exact function. This is clear in Fig. 2, where the expansion for $k_{0}=0.4$ gives a better location of that minimum.

In this sense, the asymmetric expansion should be combined with a sort of iterative procedure to locate the position of these equilibrium points. Starting with a given center $\left(k_{0}, h_{0}\right)$, we estimate the location of such an equilibrium point, then we can use this point as a new center to recalculate the coefficients of 


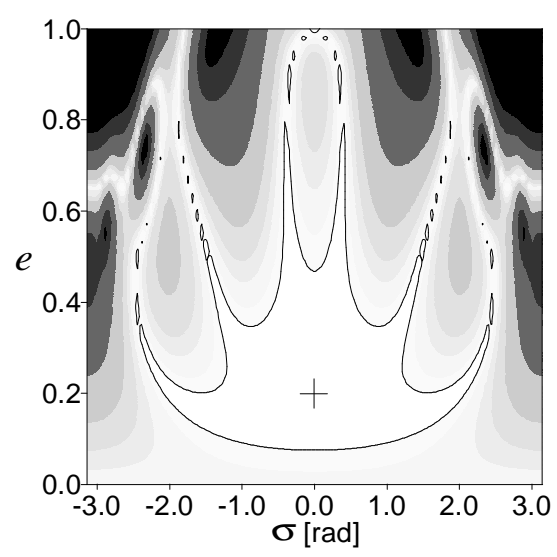

(a)
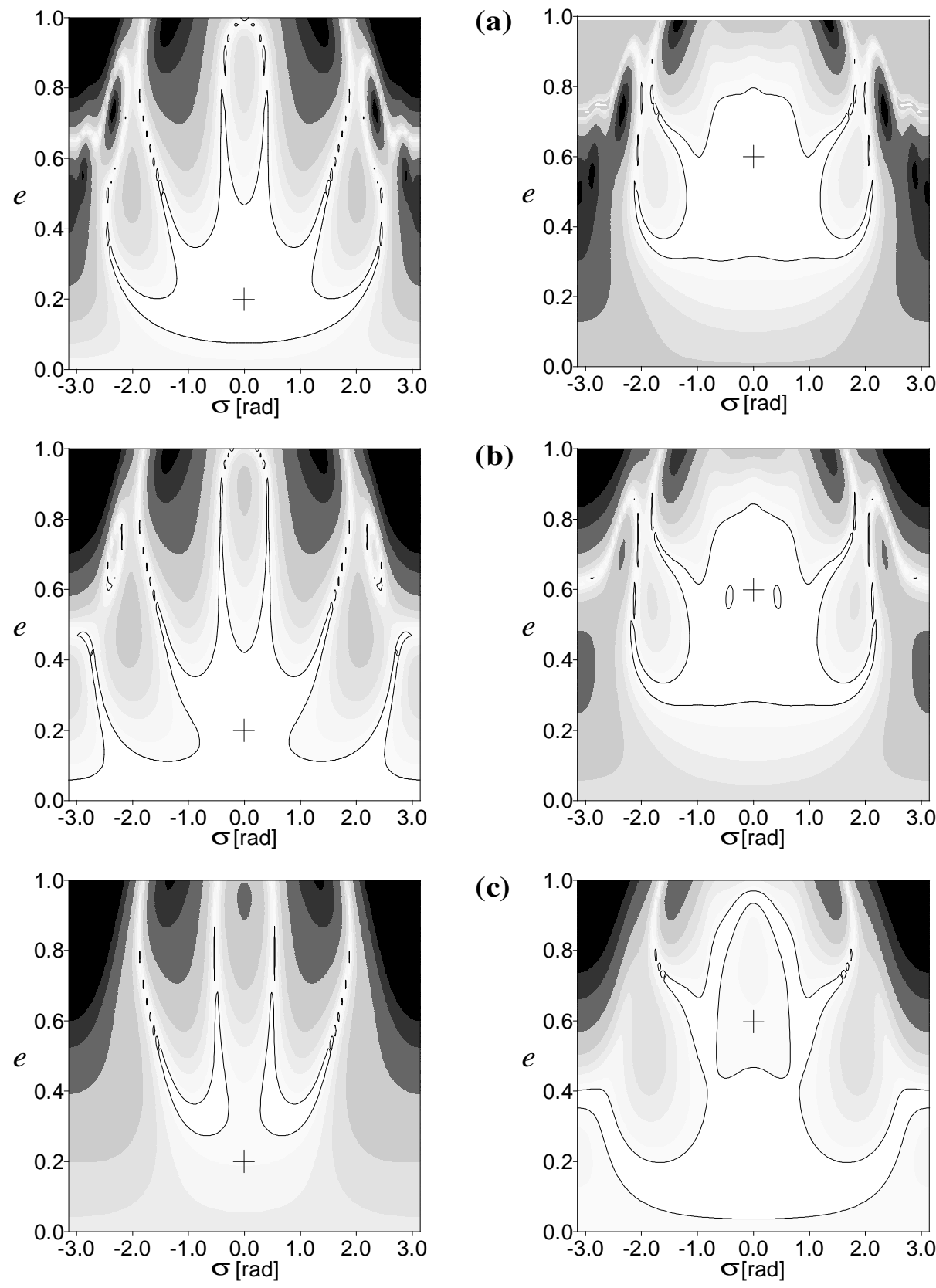

(b)

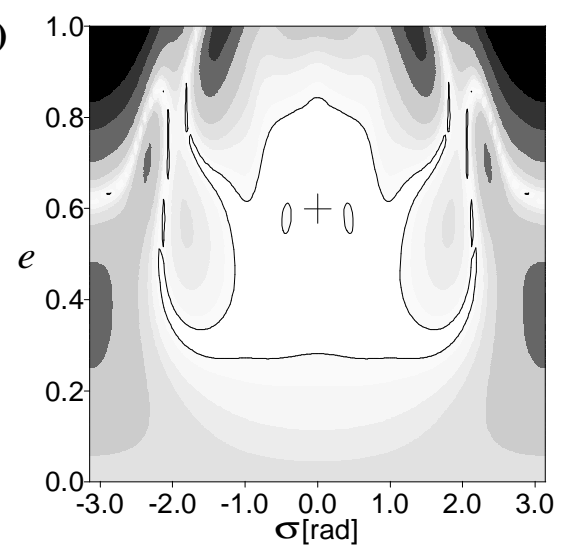

(c)

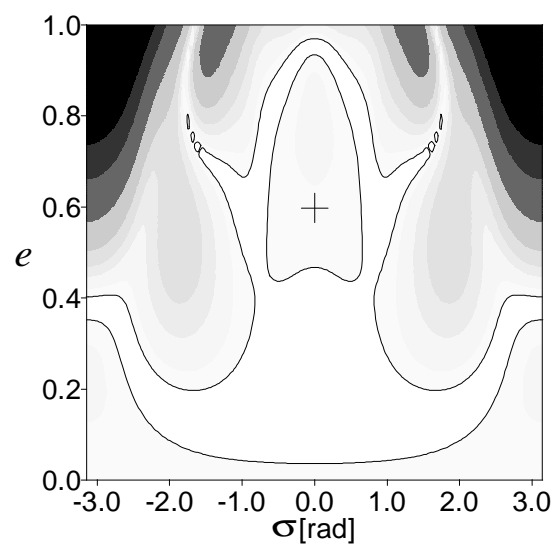

Fig. 3a-c. Relative difference in the plane $e, \sigma$ between the exact disturbing function and the asymmetric expansion for two different centers of expansion, corresponding to the $2 / 1$ resonance. Left: $k_{0}=0.2, h_{0}=0, a_{0}=3.275$ AU. Rigth: $k_{0}=0.6, h_{0}=0, a_{0}=3.275 \mathrm{AU}$ (the cross indicates the center of expansion). The results are presented for three different inclinations, considering $\sigma_{z}=0$ : a Case $I=0^{\circ}$, b Case $I=15^{\circ}$, and c Case $I=30^{\circ}$. In all figures, the variables of the disturbing body have the same values as in Fig. 1 . The white region corresponds to a difference below $1 \%$. Note that the approximation becomes poor as the inclination increases, and can be better in other region than the center of expansion.

the expansion, and so on. This kind of procedure was already applied, for example, by Ferraz-Mello et al. (1993) to compute the locus of corotation solutions in many asteroidal resonances, using the planar expansion. We stress the fact that, even if we can locate the exact position of a corotation, the expansion will be valid only in neighbourhood of that corotation (which is the actual center of the expansion). Thus, we should not expect to obtain a good representation of the whole secular resonance.

In order to reproduce the locus of secular and secondary resonances inside first-order mean motion resonances, some tests were recently made by Nesvorný (1997), using the spatial asym- metric expansion of this paper. He combined the expansion with the perturbative method of Henrard (1990) and succeded in obtain the position of some secular and secondary resonances, as well as the Kozai resonance, inside the $2 / 1,3 / 2$ and $4 / 3$ resonances. As an example, we presented in Fig. 4 the location of the stable equilibrium of the Kozai resonance (together with its separatrixes), at the pericentric branch of the $2 / 1$ resonance. It is also shown the position of the $\nu_{16}$ secular resonance. These calculations were made taking different asymmetric expansions around points $\left(k_{0}, a_{0}\right)$ over the pericentric branch. We can compare this figure with Fig. 6 of Morbidelli \& Moons (1993) or 


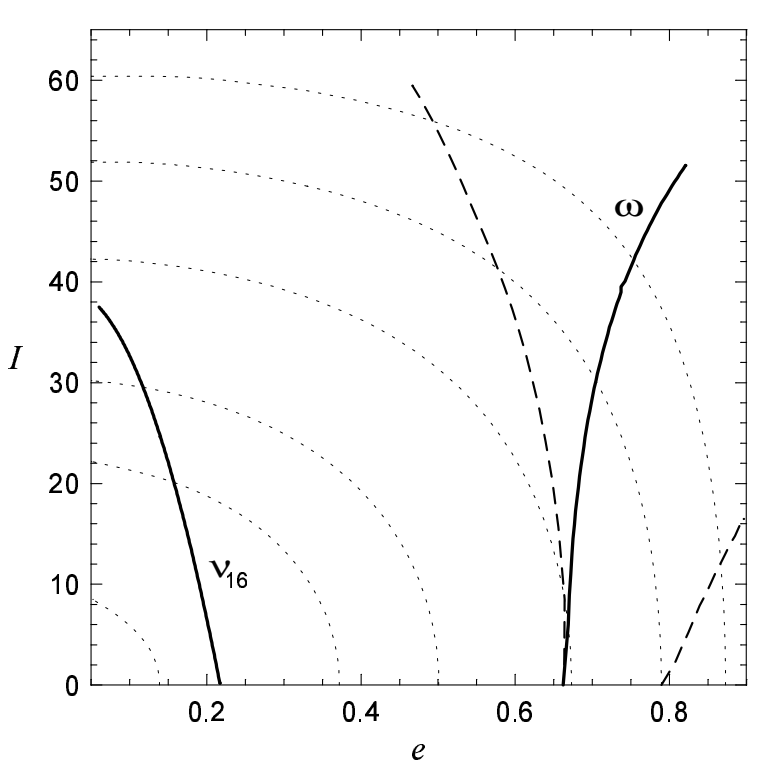

Fig. 4. The continous lines represent the location of the stable equilibrium points of the Kozai resonance ( $\omega$ curve) and the $\nu_{16}$ secular resonance, at the pericentric branch of the $2 / 1$ mean motion resonance ( $\sigma=0$ and $\sigma_{z}=0$ ). The dashed lines are the separatrixes of the Kozai resonance. The calculations were made using the asymmetric expansion of this paper and considering $a^{\prime}=5.2 \mathrm{AU}, e^{\prime}=0.048, I^{\prime}=1.2^{\circ}$, and $\sigma^{\prime}=\sigma_{z}^{\prime}=0$. Dotted lines are different levels of the constant $N=(\mu a)^{1 / 2}\left(2-\left(1-e^{2}\right)^{1 / 2} \cos I\right)$.

with Fig. 3 of Henrard et al. (1995) and see that the agreement is very good.

\section{Conclusions}

We have presented the asymmetric expansion of the averaged resonant disturbing function in the non-planar problem. Since this expansion is done around a fixed point in the $k, h$ plane and truncated at $\mathscr{O}\left(e^{\prime 3}\right)$, it approximates well the disturbing function only in a neighborhood of that point, and we have shown that this neighborhood around the center of expansion can be wide enough. Thus, we can apply it to problems in which the amplitude of libration is moderate. Recall that any application must be also limited in inclination, since we have expanded the function around zero inclinations.

Currently, this asymmetric expansion is being used in the construction of a non-planar symplectic mapping for the firstorder asteroidal resonances, which extends the planar mapping used by Ferraz-Mello (1997). It is also being used in the formulation of an analytic theory of the high-eccentricity libration dynamics (Ferraz-Mello et al., 1997).

The asymmetric expansion has been already shown to be an useful tool for the expansion of the disturbing potential acting on a resonant asteroid, and the expansion presented in this paper may be useful in the analytic studies of the spatial resonant problems in high eccentricities.
FORTRAN and C codes to compute the coefficients of this expansion are available by anonymous ftp at:

ftp://chaos1.iagusp.usp.br/users/ftp/pub/highecc.

Acknowledgements. We are indebted to Dr. D. Nesvorný for his helpful comments and suggestions. This work was supported by the São Paulo State Science Foundation (FAPESP).

\section{References}

Brouwer D. and Clemence G., 1961, Methods of Celestial Mechanics, Academic Press, N.Y.

Ferraz-Mello S., 1987, A\&A 183, 397-402

Ferraz-Mello S., 1988, Astron. J. 96, 400-408

Ferraz-Mello S., 1989, Celest. Mech. 45, 65-68

Ferraz-Mello S., 1994, Cel. Mech. Dyn. Astr. 58, 37-52

Ferraz-Mello S., 1997, Cel. Mech. Dyn. Astr. (in press)

Ferraz-Mello S. and Klafke J.C., 1991, in: Predictability, Stability and Chaos in N-Body Dynamical Systems (A.E. Roy ed.), Plenum Press, N.Y., 177-184

Ferraz-Mello S. and Sato M., 1989, A\&A 225, 541-547 ( FMS89)

Ferraz-Mello S, Tsuchida M. and Klafke J.C., 1993, Cel. Mech. Dyn. Ast. 55, 25-45

Ferraz-Mello S., Simula A. and Giordano C., 1997, in preparation Gallardo T. and Ferraz-Mello S., 1995, Cel. Mech. Dyn. Astr. 62, 145-165

Henrard J., 1990, Celest. Mech. 49, 43-67

Henrard J., Watanabe N. and Moons M., 1995, Icarus 115, 336-346

Moons M., 1993, Dep. of Math. Rep. 93-19, F.U.N.D.P., Namur

Moons M., 1994, Cel. Mech. Dyn. Astr. 60, 173-186

Morbidelli A. and Moons M., 1993, Icarus 102, 316-332

Murray C.D., 1986, Icarus 65, 70-82

Nesvorný D., 1997, Ph. D. Thesis, University of São Paulo

Oliveira M., 1995, M. S. Dissertation, University of São Paulo

Roig F., 1997, M. S. Dissertation, University of São Paulo

Yokoyama T., 1994, Cel. Mech. Dyn. Astr. 60, 387-400

Wintner A., 1941, The Analytical Foundations of Celestial Mechanics, Princeton Univ. Press, New Jersey

Wisdom J., 1987, Icarus 72, 241-275

Woltjer J., 1928, Ann. Sterr. Leiden 16(3)

This article was processed by the author using Springer-Verlag $\mathrm{LAT}_{\mathrm{E}} \mathrm{X}$ A\&A style file $L-A A$ version 3 . 


\section{A. The functions $W_{1}, W_{2}$ and their derivatives}

We give here the explicit formulas to calculate the functions $W_{1}, W_{2}$ and their derivatives using the closed formulas of FMS89.

First, we start from Eqs. (46), and to simplify the notation we define

$c=\cos (u-\sigma)$

$s=\sin (u-\sigma)$

so

$W_{1}=c-k+h T$

$W_{2}=s+h+k T$

Thus, the first derivatives are:

$\frac{d W_{1}}{d h}=\frac{d c}{d h}+T+h \frac{d T}{d h}$

$\frac{d W_{1}}{d k}=\frac{d c}{d k}-1+h \frac{d T}{d k}$

$\frac{d W_{2}}{d h}=\frac{d s}{d h}+1+k \frac{d T}{d h}$

$\frac{d W_{2}}{d k}=\frac{d s}{d k}+T+k \frac{d T}{d k}$

And the second derivatives are:

$\frac{d^{2} W_{1}}{d h^{2}}=\frac{d^{2} c}{d h^{2}}+2 \frac{d T}{d h}+h \frac{d^{2} T}{d h^{2}}$

$\frac{d^{2} W_{1}}{d k^{2}}=\frac{d^{2} c}{d k^{2}}+h \frac{d^{2} T}{d k^{2}}$

$\frac{d^{2} W_{1}}{d h d k}=\frac{d^{2} c}{d h d k}+\frac{d T}{d k}+h \frac{d^{2} T}{d h d k}$

$\frac{d^{2} W_{2}}{d h^{2}}=\frac{d^{2} s}{d h^{2}}+k \frac{d^{2} T}{d h^{2}}$

$\frac{d^{2} W_{2}}{d k^{2}}=\frac{d^{2} s}{d k^{2}}+2 \frac{d T}{d k}+k \frac{d^{2} T}{d k^{2}}$

$\frac{d^{2} W_{2}}{d h d k}=\frac{d^{2} s}{d h d k}+\frac{d T}{d h}+k \frac{d^{2} T}{d h d k}$

A.1. Derivatives of $\cos (u-\sigma)$ and $\sin (u-\sigma)$

To find the derivatives of $c$ ans $s$, we introduce the quantity

$D=(1-e \cos u)^{-1}=(1-k c+h s)^{-1}$

which in terms of the first Eq. (20) can be written as $D=$ $g_{1}^{-1 / 2}$. Then, after some algebra, the direct differentiation of Kepler's equation gives the following results for the first derivatives of $c$ and $s$ :

$$
\begin{aligned}
& \frac{d c}{d h}=-g_{1}^{-1 / 2} s c \\
& \frac{d s}{d h}=g_{1}^{-1 / 2} c^{2} \\
& \frac{d c}{d k}=-g_{1}^{-1 / 2} s^{2} \\
& \frac{d s}{d k}=g_{1}^{-1 / 2} s c
\end{aligned}
$$

To calculate the second derivatives of $c$ and $s$ we use the relations

$\frac{d D}{d k}=D^{3}(c-k)$

$\frac{d D}{d h}=-D^{3}(s+h)$

then we write

$\frac{d^{2} c}{d h^{2}}=g_{1}^{-3 / 2}(s+h) s c+g_{1}^{-1}\left(s^{2}-c^{2}\right) c=-\frac{d^{2} s}{d h d k}$

$\frac{d^{2} c}{d k^{2}}=-g_{1}^{-3 / 2}(c-k) s^{2}-2 g_{1}^{-1} s^{2} c$

$\frac{d^{2} s}{d h^{2}}=-g_{1}^{-3 / 2}(s+h) c^{2}-2 g_{1}^{-1} c^{2} s$

$\frac{d^{2} s}{d k^{2}}=g_{1}^{-3 / 2}(c-k) s c+g_{1}^{-1}\left(c^{2}-s^{2}\right) s=-\frac{d^{2} c}{d k d h}$

\section{A.2. Derivatives of $T$}

To find the derivatives of $T$ we write Eq. (47) in the form

$T=T_{1} T_{2}$

where

$T_{1}=\frac{1}{e^{2}}\left(\sqrt{1-e^{2}}-1\right)$

$T_{2}=e \sin u$

Then, the first derivatives are:

$\frac{d T}{d h}=\frac{d T_{1}}{d e} \frac{d e}{d h} T_{2}+\frac{d T_{2}}{d h} T_{1}$

$\frac{d T}{d k}=\frac{d T_{1}}{d e} \frac{d e}{d k} T_{2}+\frac{d T_{2}}{d k} T_{1}$

and the second derivatives are:

$\begin{aligned} \frac{d^{2} T}{d h^{2}} & =\frac{d^{2} T_{1}}{d h^{2}} T_{2}+2 \frac{d T_{1}}{d h} \frac{d T_{2}}{d h}+\frac{d^{2} T_{2}}{d h^{2}} T_{1} \\ \frac{d^{2} T}{d k^{2}} & =\frac{d^{2} T_{1}}{d k^{2}} T_{2}+2 \frac{d T_{1}}{d k} \frac{d T_{2}}{d k}+\frac{d^{2} T_{2}}{d k^{2}} T_{1} \\ \frac{d^{2} T}{d h d k} & =\frac{d^{2} T_{1}}{d h d k} T_{2}+\frac{d T_{1}}{d k} \frac{d T_{2}}{d h}+\frac{d T_{1}}{d h} \frac{d T_{2}}{d k}+\frac{d^{2} T_{2}}{d h d k} T_{1}\end{aligned}$

Now, from Eqs. (A10) the derivatives of $T_{1}$ with respect to $e$ are:

$\frac{d T_{1}}{d e}=-\frac{e T_{1}^{2}}{\sqrt{1-e^{2}}}$

$\frac{d^{2} T_{1}}{d e^{2}}=\frac{1}{1-e^{2}}\left[-\frac{T_{1}^{2}}{\sqrt{1-e^{2}}}+2 e^{2} T_{1}^{3}\right]$

Thus, using $e=\sqrt{k^{2}+h^{2}}$, we can write the first derivatives in the form

$\frac{d T_{1}}{d h}=-\frac{T_{1}^{2}}{\sqrt{1-e^{2}}} h$

$\frac{d T_{1}}{d k}=-\frac{T_{1}^{2}}{\sqrt{1-e^{2}}} k$ 
and for the second derivatives we have

$$
\begin{aligned}
\frac{d^{2} T_{1}}{d h^{2}} & =\left(2 T_{1}^{3}-\frac{T_{1}^{2}}{\sqrt{1-e^{2}}}\right) \frac{h^{2}}{1-e^{2}}-\frac{T_{1}^{2}}{\sqrt{1-e^{2}}} \\
\frac{d^{2} T_{1}}{d k^{2}} & =\left(2 T_{1}^{3}-\frac{T_{1}^{2}}{\sqrt{1-e^{2}}}\right) \frac{k^{2}}{1-e^{2}}-\frac{T_{1}^{2}}{\sqrt{1-e^{2}}} \\
\frac{d^{2} T_{1}}{d h d k} & =\left(2 T_{1}^{3}-\frac{T_{1}^{2}}{\sqrt{1-e^{2}}}\right) \frac{h k}{1-e^{2}}
\end{aligned}
$$

The first and second derivatives of $T_{2}$ can be calculated from the direct differentiation of Kepler's equation, obtaining

$$
\begin{aligned}
& \frac{d T_{2}}{d h}=D c \\
& \frac{d T_{2}}{d k}=D s
\end{aligned}
$$

and

$\frac{d^{2} T_{2}}{d h^{2}}=-D^{3}(s+h) c-D^{2} s c$

$\frac{d^{2} T_{2}}{d k^{2}}=D^{3}(c-k) s+D^{2} s c$

$\frac{d^{2} T_{2}}{d h d k}=-D^{3}(s+h) s+D^{2} c^{2}$

All these formulas are not singular when $e=0$. For the first Eq. (A10) we have an indetermination at $e=0$ which can be removed multiplying and dividing $T_{1}$ by $1+\sqrt{1-e^{2}}$. Thus we obtain

$T_{1}=-\frac{1}{1+\sqrt{1-e^{2}}}$

\section{B. Coefficients of the expansion in $k, h$ and $\alpha$}

The explicit form of the coefficients calculated in Sect. 4 are as follows.

\section{B.1. Term of order zero}

The derivatives of the direct part are:

$$
\begin{aligned}
f_{0 h} & =-\frac{1}{2} f_{0}^{3}\left[\alpha^{2} g_{1 h}-2 \alpha g_{4 h}\right] \\
f_{0 k} & =-\frac{1}{2} f_{0}^{3}\left[\alpha^{2} g_{1 k}-2 \alpha g_{4 k}\right] \\
f_{0 \alpha} & =-\frac{1}{2} f_{0}^{3}\left[2 \alpha g_{1}-2 g_{4}\right] \\
f_{0 h h} & =3 f_{0}^{-1}\left(f_{0 h}\right)^{2}-\frac{1}{2} f_{0}^{3}\left[\alpha^{2} g_{1 h h}-2 \alpha g_{4 h h}\right] \\
f_{0 k k} & =3 f_{0}^{-1}\left(f_{0 k}\right)^{2}-\frac{1}{2} f_{0}^{3}\left[\alpha^{2} g_{1 k k}-2 \alpha g_{4 k k}\right] \\
f_{0 \alpha \alpha} & =3 f_{0}^{-1}\left(f_{0 \alpha}\right)^{2}-f_{0}^{3} g_{1} \\
f_{0 h k} & =3 f_{0}^{-1} f_{0 h} f_{0 k}-\frac{1}{2} f_{0}^{3}\left[\alpha^{2} g_{1 h k}-2 \alpha g_{4 h k}\right] \\
f_{0 h \alpha} & =3 f_{0}^{-1} f_{0 h} f_{0 \alpha}-\frac{1}{2} f_{0}^{3}\left[2 \alpha g_{1 h}-2 g_{4 h}\right] \\
f_{0 k \alpha} & =3 f_{0}^{-1} f_{0 k} f_{0 \alpha}-\frac{1}{2} f_{0}^{3}\left[2 \alpha g_{1 k}-2 g_{4 k}\right]
\end{aligned}
$$

The derivatives of the indirect part are:

$$
\begin{aligned}
f_{0 h}^{1} & =-\alpha g_{4 h} \\
f_{0 k}^{1} & =-\alpha g_{4 k} \\
f_{0 \alpha}^{1} & =-g_{4} \\
f_{0 h h}^{1} & =-\alpha g_{4 h h} \\
f_{0 k k}^{1} & =-\alpha g_{4 k k} \\
f_{0 \alpha \alpha}^{1} & =0 \\
f_{0 h k}^{1} & =-\alpha g_{4 h k} \\
f_{0 h \alpha}^{1} & =-g_{4 h} \\
f_{0 k \alpha}^{1} & =-g_{4 k}
\end{aligned}
$$

\section{B.2. Terms in $e^{\prime}$}

The derivatives of the direct part are:

$$
\begin{aligned}
f_{1 h} & =3 f_{0}^{-1} f_{0 h} f_{1}+f_{0}^{3}\left[-\alpha g_{4 h} \cos p Q+2 \alpha g_{5 h} \sin p Q\right] \\
f_{1 k} & =3 f_{0}^{-1} f_{0 k} f_{1}+f_{0}^{3}\left[-\alpha g_{4 k} \cos p Q+2 \alpha g_{5 k} \sin p Q\right] \\
f_{1 \alpha} & =3 f_{0}^{-1} f_{0 \alpha} f_{1}+f_{0}^{3}\left[-g_{4} \cos p Q+2 g_{5} \sin p Q\right]
\end{aligned}
$$

$f_{2 h}=-3 f_{0}^{-1} f_{0 h} f_{2}+f_{0}^{3}\left[\alpha g_{4 h} \sin p Q+2 \alpha g_{5 h} \cos p Q\right]$ $f_{2 k}=-3 f_{0}^{-1} f_{0 k} f_{2}+f_{0}^{3}\left[\alpha g_{4 k} \sin p Q+2 \alpha g_{5 k} \cos p Q\right]$ $f_{2 \alpha}=-3 f_{0}^{-1} f_{0 \alpha} f_{2}+f_{0}^{3}\left[g_{4} \sin p Q+2 g_{5} \cos p Q\right]$

The derivatives of the indirect part are:

$f_{1 h}^{1}=-2 \alpha\left(g_{4 h} \cos p Q+g_{5 h} \sin p Q\right)$

$f_{1 k}^{1}=-2 \alpha\left(g_{4 k} \cos p Q+g_{5 k} \sin p Q\right)$

$f_{1 \alpha}^{1}=-2\left(g_{4} \cos p Q+g_{5} \sin p Q\right)$

$f_{2 h}^{1}=2 \alpha\left(g_{4 h} \sin p Q-g_{5 h} \cos p Q\right)$

$f_{2 k}^{1}=2 \alpha\left(g_{4 k} \sin p Q-g_{5 k} \cos p Q\right)$

$f_{2 \alpha}^{1}=2\left(g_{4} \sin p Q-g_{5} \cos p Q\right)$

\section{B.3. Terms in $\eta^{2}$}

The derivatives of the direct part are:

$$
\begin{aligned}
f_{3 h} & =-6 f_{0}^{2} f_{0 h} \alpha g_{4}-2 f_{0}^{3} \alpha g_{4 h} \\
f_{3 k} & =-6 f_{0}^{2} f_{0 k} \alpha g_{4}-2 f_{0}^{3} \alpha g_{4 k} \\
f_{3 \alpha} & =-6 f_{0}^{2} f_{0 \alpha} \alpha g_{4}-2 f_{0}^{3} g_{4} \\
f_{4 h} & =6 f_{0}^{2} f_{0 h} \alpha g_{6}+2 f_{0}^{3} \alpha g_{6 h} \\
f_{4 k} & =6 f_{0}^{2} f_{0 k} \alpha g_{6}+2 f_{0}^{3} \alpha g_{6 k} \\
f_{4 \alpha} & =6 f_{0}^{2} f_{0 \alpha} \alpha g_{6}+2 f_{0}^{3} g_{6} \\
f_{5 h} & =-6 f_{0}^{2} f_{0 h} \alpha g_{7}-2 f_{0}^{3} \alpha g_{7 h} \\
f_{5 k} & =-6 f_{0}^{2} f_{0 k} \alpha g_{7}-2 f_{0}^{3} \alpha g_{7 k} \\
f_{5 \alpha} & =-6 f_{0}^{2} f_{0 \alpha} \alpha g_{7}-2 f_{0}^{3} g_{7}
\end{aligned}
$$


The derivatives of the indirect part are:

$$
\begin{aligned}
& f_{3 h}^{1}=2 \alpha g_{4 h} \\
& f_{3 k}^{1}=2 \alpha g_{4 k} \\
& f_{3 \alpha}^{1}=2 g_{4} \\
& f_{4 h}^{1}=-2 \alpha g_{6 h} \\
& f_{4 k}^{1}=-2 \alpha g_{6 k} \\
& f_{4 \alpha}^{1}=-2 g_{6} \\
& f_{5 h}^{1}=2 \alpha g_{7 h} \\
& f_{5 k}^{1}=2 \alpha g_{7 k} \\
& f_{5_{\alpha}}^{1}=2 g_{7}
\end{aligned}
$$

\section{B.4. Oblateness perturbation}

For the zero order term the derivatives are:

$$
\begin{aligned}
\mathcal{R}_{0 h}^{J} & =\frac{3}{4} \alpha^{-3} g_{1}^{-5 / 2} g_{1 h} \\
\mathcal{R}_{0 k}^{J} & =\frac{3}{4} \alpha^{-3} g_{1}^{-5 / 2} g_{1 k} \\
\mathcal{R}_{0 \alpha}^{J} & =\frac{3}{2} \alpha^{-4} g_{1}^{-3 / 2} \\
\mathcal{R}_{0 h h}^{J} & =\frac{3}{8} \alpha^{-3} g_{1}^{-5 / 2}\left[-5 g_{1}^{-1}\left(g_{1 h}\right)^{2}+2 g_{1 h h}\right] \\
\mathcal{R}_{0 k k}^{J} & =\frac{3}{8} \alpha^{-3} g_{1}^{-5 / 2}\left[-5 g_{1}^{-1}\left(g_{1 k}\right)^{2}+2 g_{1 k k}\right] \\
\mathcal{R}_{0 \alpha \alpha}^{J} & =6 \alpha^{-5} g_{1}^{-3 / 2} \\
\mathcal{R}_{0 h k}^{J} & =\frac{3}{8} \alpha^{-3} g_{1}^{-5 / 2}\left[-5 g_{1}^{-1} g_{1 h} g_{1 k}+2 g_{1 h k}\right] \\
\mathcal{R}_{0 h \alpha}^{J} & =\frac{9}{4} \alpha^{-4} g_{1}^{-5 / 2} g_{1 h} \\
\mathcal{R}_{0 k \alpha}^{J} & =\frac{9}{4} \alpha^{-4} g_{1}^{-5 / 2} g_{1 k}
\end{aligned}
$$

For the terms in the inclination, the derivatives of the coefficients are:

$$
\begin{aligned}
& \mathcal{R}_{2 h}^{J}=12\left[\mathcal{R}_{0 h}^{J} g_{1}^{-1} g_{8}+\mathcal{R}_{0}^{J} g_{1}^{-2}\left(g_{8 h} g_{1}-g_{8} g_{1 h}\right)\right] \\
& \mathcal{R}_{2 k}^{J}=12\left[\mathcal{R}_{0 k}^{J} g_{1}^{-1} g_{8}+\mathcal{R}_{0}^{J} g_{1}^{-2}\left(g_{8 k} g_{1}-g_{8} g_{1 k}\right)\right] \\
& \mathcal{R}_{2 \alpha}^{J}=12 \mathcal{R}_{0 \alpha}^{J} g_{1}^{-1} g_{8}
\end{aligned}
$$

and

$$
\begin{aligned}
& \mathcal{R}_{3 h}^{J}=-12\left[\mathcal{R}_{0 h}^{J} g_{1}^{-1} g_{9}+\mathcal{R}_{0}^{J} g_{1}^{-2}\left(g_{9 h} g_{1}-g_{9} g_{1 h}\right)\right] \\
& \mathcal{R}_{3 k}^{J}=-12\left[\mathcal{R}_{0 k}^{J} g_{1}^{-1} g_{9}+\mathcal{R}_{0}^{J} g_{1}^{-2}\left(g_{9 k} g_{1}-g_{9} g_{1 k}\right)\right] \\
& \mathcal{R}_{3 \alpha}^{J}=-12 \mathcal{R}_{0 \alpha}^{J} g_{1}^{-1} g_{9}
\end{aligned}
$$

\section{Expansion for low eccentricities}

If we want an expansion around a point with $e_{0}<0.6627$ we can use the series representation of the functions $g_{2}$ and $g_{3}$ :

$$
\begin{aligned}
& g_{2}=\operatorname{Re}\left[\sum_{n=0}^{\infty} P_{n}\left(e^{2}\right)(k+i h)^{n} \exp i n(p+q) Q\right] \\
& g_{3}=\operatorname{Im}\left[\sum_{n=0}^{\infty} P_{n}^{\prime}\left(e^{2}\right)(k+i h)^{n} \exp i n(p+q) Q\right]
\end{aligned}
$$

where $P_{n}, P_{n}^{\prime}$ are known polynomials (see FMS89). Thus, the functions $W_{1}, W_{2}$ and their derivatives depend only on $Q$, and the average of $R$ is computed as

$R^{*}=\frac{1}{2 \pi} \int_{0}^{2 \pi} R[Q] d Q$

In the particular case when the expansion is done at the origin $k_{0}=h_{0}=0$ (Laplacian expansion), we have $g_{2}=1$ and $g_{3}=0$. Thus, we replace Eqs. (46) by

$$
\begin{aligned}
& W_{1}=\cos [(p+q) Q] \\
& W_{2}=\sin [(p+q) Q]
\end{aligned}
$$

Parity rules are also applied in this case so the average is computed as

$$
R^{*}=\frac{1}{\pi} \int_{0}^{\pi} R[Q] d Q
$$

